

\title{
Charge transfer and redistribution at interfaces \\ between metals and 2D materials
}

\author{
Menno Bokdam
}




\section{Graduation committee:}

\begin{tabular}{|c|c|c|}
\hline Prof. dr. Han Gardeniers & University of Twente & chairman \\
\hline Prof. dr. Paul J. Kelly & University of Twente & promotor \\
\hline dr. Geert Brocks & University of Twente & assistant promotor \\
\hline Prof. dr. Kristian S. Thygesen & Technical University of Denmark & \\
\hline Prof. dr. ir. Bart J. van Wees & University of Groningen & \\
\hline dr. Petr A. Khomyakov & IBM Research Zurich & \\
\hline Prof. dr. ir. Bene Poelsema & University of Twente & \\
\hline Prof. dr. ir. Hans Hilgenkamp & University of Twente & \\
\hline
\end{tabular}
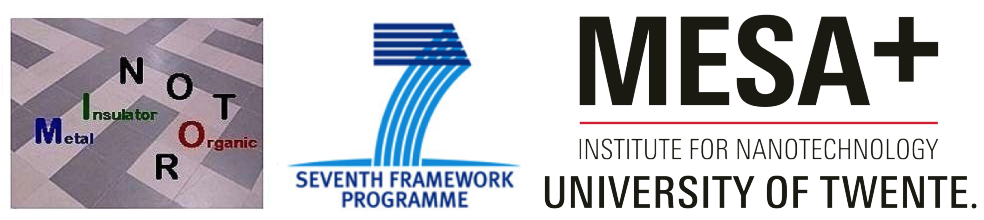

This work was supported financially by the European project MINOTOR, grant no. FP7-NMP-228424. The use of supercomputer facilities was sponsored by the "Stichting Nationale Computerfaciliteiten (NCF)", financially supported by the "Nederlandse Organisatie voor Wetenschappelijk Onderzoek (NWO)".

Charge transfer and redistribution at interfaces between metals and 2D materials (Ladingsoverdracht en -herverdeling op grensvlakken tussen metalen en 2D materialen)

Menno Bokdam

First print

PhD thesis University of Twente, Enschede

ISBN: 978-90-365-1133-9

DOI: $10.3990 / 1.9789036511339$

Copyright (c) M. Bokdam, 2013

Published by: M. Bokdam

Printed by: Gildeprint Drukkerijen - Enschede

Cover: "The redistribution of electrons in the 2D materials system: Graphene on top of hexagonal Boron Nitride. When a graphene layer is placed on top of a layer of $h-B N$, electrons are moved from the red regions into the blue regions. The two layers are rotated by an angle of $13^{\circ}$ relative to each other, thereby creating a moiré pattern." 


\title{
CHARGE TRANSFER AND REDISTRIBUTION AT INTERFACES BETWEEN METALS AND 2D MATERIALS
}

\author{
PROEFSCHRIFT
}

\author{
ter verkrijging van \\ de graad van doctor aan de Universiteit Twente, \\ op gezag van de rector magnificus, \\ Prof. dr. H. Brinksma, \\ volgens besluit van het College voor Promoties \\ in het openbaar te verdedigen \\ op vrijdag 15 november 2013 om 16:45 uur
}

door

\section{Menno Bokdam}

geboren op 10 oktober 1985

te Soest, Nederland. 
Dit proefschrift is goedgekeurd door:

Prof. dr. Paul J. Kelly (promotor)

dr. Geert Brocks (assistent promotor) 
Lectori salutem 



\section{Contents}

1 Introduction 1

1.1 The world of electrons ...................... 1

1.2 Interfaces are the key factors . . . . . . . . . . . . 3

1.3 Novel 2D materials . . . . . . . . . . . . . . . . . . 6

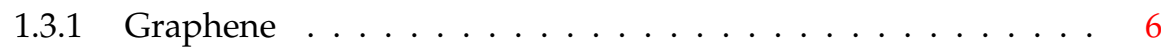

1.3.2 Hexagonal Boron Nitride . . . . . . . . . . . . . . 8

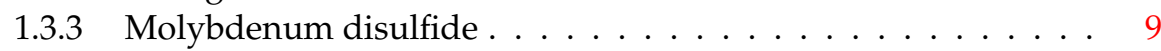

1.4 Charge equilibration across multiple layers . . . . . . . . . . . . 9

2 Methods 11

2.1 Density Functional Theory . . . . . . . . . . . . . . . . . . . 11

2.2 Plane wave pseudopotential method . . . . . . . . . . . . . . . . . . . . . . . . . 13

2.3 Projector Augmented Wave method . . . . . . . . . . . . . . . . . . 16

2.4 Exchange and Correlation functionals . . . . . . . . . . . . . . . . 19

2.4.1 Local Density Approximation \& Generalized-Gradient Approx-

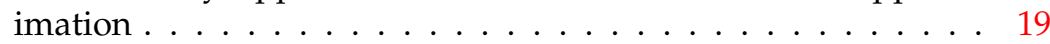

2.4 .2 Beyond local functionals . . . . . . . . . . . . . . 20

2.5 The $\mathrm{G}_{0} \mathrm{~W}_{0}$ approximation . . . . . . . . . . . . 22

3 Fermi level pinning by integer charge transfer at electrode-organic semiconductor interfaces 23

3.1 Introduction . . . . . . . . . . . . . . . . 23

3.2 Integer charging model . . . . . . . . . . . . . . . . 24

3.3 DFT parametrisation . . . . . . . . . . . . . . . 27

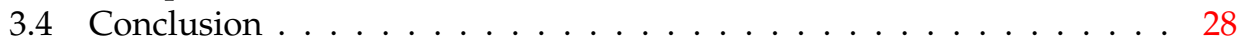

4 Electrostatic doping of graphene through ultrathin hexagonal boron nitride films $\quad 29$

4.1 Introduction . . . . . . . . . . . . . . . . . . . . . 29

4.2 Computational Details . . . . . . . . . . . . . . . 30

4.3 Intrinsic Doping . . . . . . . . . . . . . . . . . . . 32

4.4 External Field . . . . . . . . . . . . . . . . . . . . . . . . . . . . . . . . . . . . . .

4.5 Model . . . . . . . . . . . . . . . . . . . 34

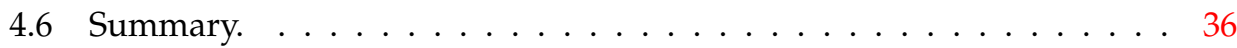


5 Field effect doping of graphene in metal|dielectric|graphene heterostructures: a model based upon first-principles calculations

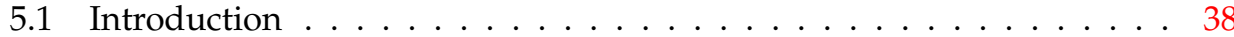

5.2 Electrostatic doping model . . . . . . . . . . . . . . . . . 39

5.3 Computational details . . . . . . . . . . . . . . . . . . 43

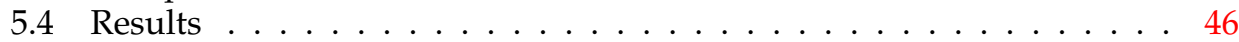

5.4.1 Potential steps at metal $\mid h$-BN and $h$-BN $\mid$ graphene interfaces . . 48

5.4.2 Fermi level shifts in metal $\mid h$-BN $\mid$ graphene heterostructures . . . 53

5.5 Summary and conclusions . . . . . . . . . . . . . . . 57

6 Large potential steps at weakly interacting metal|insulator interfaces $\quad 59$

6.1 Introduction . . . . . . . . . . . . . . . . . . 59

6.2 DFT calculations . . . . . . . . . . . . . . . . 60

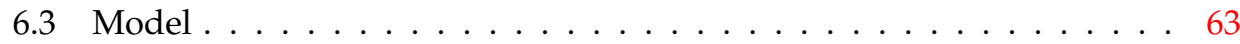

$6.4 \mathrm{Cu}(111) \mid h$-BN interface . . . . . . . . . . . . . . . . . . 64

6.5 Interface potential steps . . . . . . . . . . . . . . . . 66

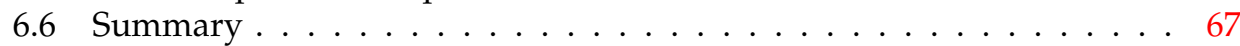

7 Schottky barriers at metal|hexagonal boron nitride interfaces: a first principles study

7.1 Introduction . . . . . . . . . . . . . . . . . . 69

7.2 Method and Computational details . . . . . . . . . . . . . 70

7.3 Results . . . . . . . . . . . . . . . . . 71

7.3.1 Metal $\mid h$-BN structures and bonding . . . . . . . . . 71

7.3.2 Metal $\mid h-\mathrm{BN}$ interface dipole . . . . . . . . . . . . . . . 75

7.3 .3 Energy level alignment . . . . . . . . . . . . . . . 80

7.3.4 Incommensurate metal $\mid h$-BN systems $\ldots \ldots \ldots \ldots$

7.4 Summary and conclusions . . . . . . . . . . . . . . . 85

8 Band gaps in incommensurable Graphene on hexagonal Boron-Nitride 87

8.1 Introduction . . . . . . . . . . . . . . . . . 87

8.2 Computational details $\ldots \ldots \ldots \ldots . \ldots . \ldots . \ldots . \ldots$

8.3 Density Functional Calculations . . . . . . . . . . . . . . . . . . . 89

8.4 GW correction . . . . . . . . . . . . . . . . . . . . . . 91

8.5 Band gap in graphene on $h$-BN $\ldots \ldots \ldots \ldots \ldots \ldots \ldots$

8.6 Tight Binding Hamiltonian $\ldots \ldots \ldots \ldots$

8.7 TB: Commensurable lattices . . . . . . . . . . . . . . . . . 93

8.8 TB: Incommensurable lattices . . . . . . . . . . . . . . . . . . . . 94

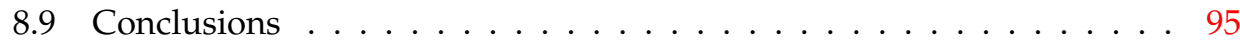

9 Intrinsic electron-hole puddles in Graphene on hexagonal Boron-Nitride 97

9.1 Introduction . . . . . . . . . . . . . . . . . . 97

9.2 Computational details $\ldots \ldots \ldots \ldots \ldots$

9.3 Graphene $\mid h$-BN binding . . . . . . . . . . . . . . . . . . 100

9.4 Commensurate structures . . . . . . . . . . . . . . . . . . . . . 101

9.5 Puddle formation . . . . . . . . . . . . . . . . . . . . . . 107

9.6 Graphene on Molybdenum Disulfide . . . . . . . . . . . . . . . . . 108

9.7 Conclusion . . . . . . . . . . . . . . . . . . . . . . . . . . . . . . 109 
A Graphene Brillouin zone sampling 111

$\begin{array}{ll}\text { Bibliography } & 115\end{array}$

$\begin{array}{ll}\text { Summary } & 127\end{array}$

$\begin{array}{ll}\text { Samenvatting } & 131\end{array}$

$\begin{array}{ll}\text { Acknowledgements/Dankwoord } & 135\end{array}$

$\begin{array}{lr}\text { Publications } & 139\end{array}$

$\begin{array}{ll}\text { Curriculum vitae } & 141\end{array}$ 


\section{1}

\section{Introduction}

\subsection{The world of electrons}

In almost any electronic device one can find a (green) printed circuit board, which links the different electronic components, such as transistors, capacitors, resistors, inductors and diodes together. In the last half century these components have been shrunk drastically in size. This makes it possible for things to be done with a smartphone that were only possible on the fastest supercomputer in the eighties. One of the main reasons for this reduction in size are the advances in photolithography of silicon based transistors. Using ultra-violet light, this technique enables silicon structures to be made with a width smaller than 50 nanometer $(0.000000050 \mathrm{~m})$. However, this process of making the silicon structures smaller and smaller cannot continue forever. A single silicon atom has a radius of roughly a tenth of a nanometer; this means that these structures are so small that the number of atoms become almost countable. ${ }^{*}$ If these silicon structures are made smaller they become more susceptible to influence from its surrounding. When these structures are used for switching, as in a transistor, the main influence is heat. A high density of transistors on a chip also means that there is a large power dissipation in a small area. When a transistor becomes hot its on/off states become less well defined and its performance deteriorates. Therefore the central processing unit in your PC is connected to a heat sink (big chunk of aluminum) and a ventilator to get rid of the heat as fast as possible.

One solution for this problem is to search for new semiconducting materials which consume less power (conduct better) and are stable on these smaller scales. This is done in the field of materials science and in this thesis. Besides exploring the properties of new materials, the main focus in this work is on the way in which two materials affect one anothers electronic properties when they are in contact. For

\footnotetext{
${ }^{*}$ Imagine that a cubic centimeter of silicon contains of the order of $10^{22}$ atoms, compared to $10^{9}$ people on this planet. A cube of $50 \mathrm{~nm}^{3}$ of silicon contains only $10^{6}$ atoms.
} 

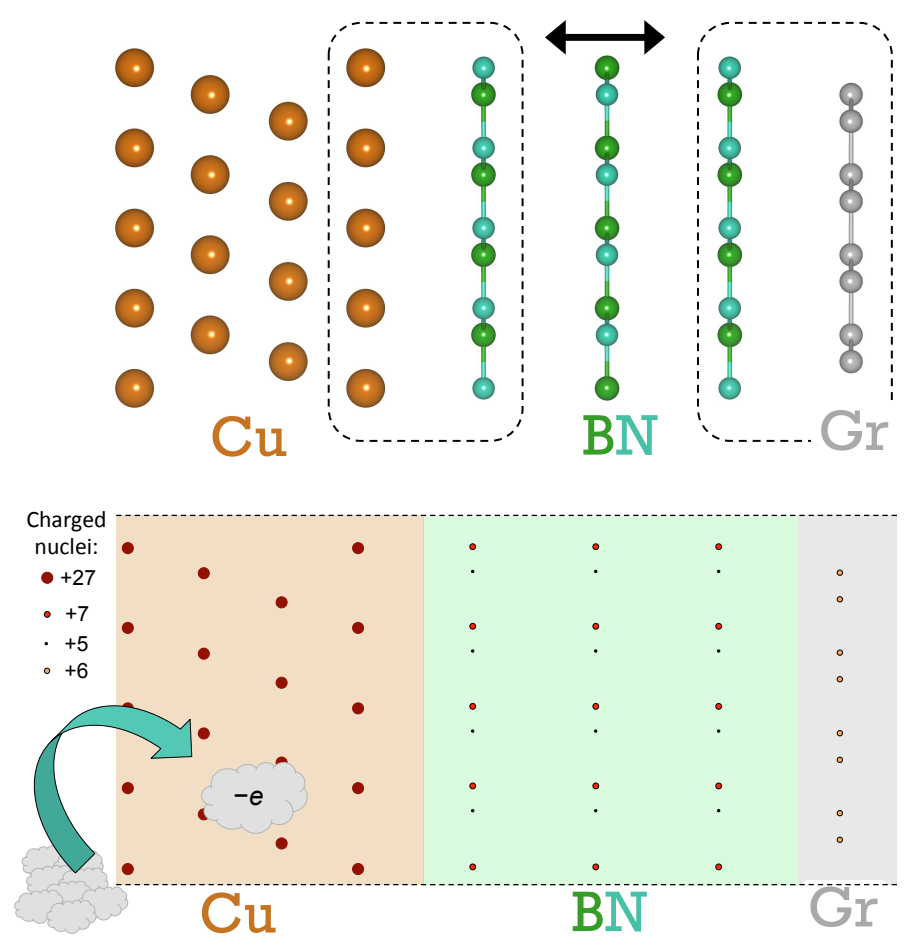

Figure 1.1: Top: Cross section of the atomic structure of a typical nanoscale multilayer structure studied in this thesis. Periodically repeating the figure at the top and bottom creates an infinite Copper surface, with three layers of hexagonal boron nitride and one graphene layer. The arrow indicates a length scale of $3 \AA$. Bottom: The positions of the positively charged atomic nuclei. The problem is to find the equilibrium distribution of electrons.

instance, a very thin film of a conducting material might have a completely different conductivity when placed on different surfaces. For these reasons (and others) the electronic effects at interfaces between novel 2D materials mutually and with metal surfaces is studied in this thesis.

Figure 1.1 (top) shows the location of the atoms (structure) in one of the systems studied in this thesis. Each atom contains a nucleus of protons and neutrons that has an electric charge of $Z e$, where $Z$ is the atomic number of the element in the periodic table. Around the nucleus $Z$ negatively charged electrons fly around in orbits. The total electric charge of a neutral atom is therefore zero. In quantum mechanics the electrons are not described as point charges, but as a cloud whose total charge is $-1 e$. The question to be answered is: "How are the electrons distributed in the landscape of Fig. 1.1 (bottom) if they are introduced into the system one by one?" The field of research which deals with this problem is called Electronic Structure Theory. To begin with, imagine that the first few electrons go directly to the most attractive copper 
nucleus that they see and stay very close to it. However as more and more electrons are added to the system they partly shield off the attractive charges of the nuclei and when they come too close they start to repel one another. After adding a certain number of electrons, it becomes energetically more favourable for an electron to be in-between two cores; the shared electron effectively creates a bond between the two atoms, which gives the structure its cohesion. This is the idea in a nut shell, the interested reader can find much more detail about the electronic structure calculations in chapter 2.

When all electrons have been added to the system the resulting structure shows strong in-plane bonds between the Boron and Nitrogen atoms and between the Carbon atoms. These bonds are indicated in Fig. 1.1 (top) as lines between the atoms. The attractive forces between different planes are much weaker and are not needed to stabilize a single two dimensional (2D) layer of this material. The right most layer, consisting of carbon atoms, is the most famous example of a 2D material. Surprisingly, it was only recently shown to exist in single layer form by K.S. Novoselov and A.K. Geim in 2004[1]. The three middle layers are layers of hexagonal Boron Nitride (h-BN). The copper atoms form a crystal structure known as Face Centred Cubic (FCC). The stacking can be compared to the optimal way to pack oranges in a crate. A FCC crystal can be cut in different ways, leading to different facets of the crystal. The copper surface(facet) on which the $h$-BN layers are stacked is called a (111) surface. This surface is atomically flat and can form an ideal ordered interface with 2D materials. The regions indicated by the dashed lines in Figure 1.1 (top) are called interfaces. In this example, there is one interface between copper and $h$-BN and another between $h$-BN and graphene. It is such interfaces which can show interesting physics. For instance, chapter 7 is concerned dipole layers which are formed at metal $\mid h-\mathrm{BN}$ interfaces and in chapter 8 the influence that the $h$-BN|graphene interface has on the electronic properties of graphene is studied.

\subsection{Interfaces are the key factors}

I would like to illustrate the physical processes controlling the interfaces studied in this thesis with an example from the field of organic electronics. In many organic based devices one can find interfaces between a metal surface and organic molecules that have been deposited on top of the metal. In many cases the work function ${ }^{\dagger}$ of the metal is changed by deposition of only a thin film of molecules. The mechanisms behind this change can be found by looking microscopically at what happens at the interface on the atomic scale.

In Figure 1.2 a) a metal|organic interface is sketched. The molecules may not be packed in an ordered way and the metal surface might have been oxidised. However, the systems we use to study the mechanisms of work function change consist of an ordered layer of molecules on top of a clean metal surface. The energy level

${ }^{\dagger}$ The work function is the energy needed to extract one electron from the metal and bring it so far away that it becomes a free electron. 

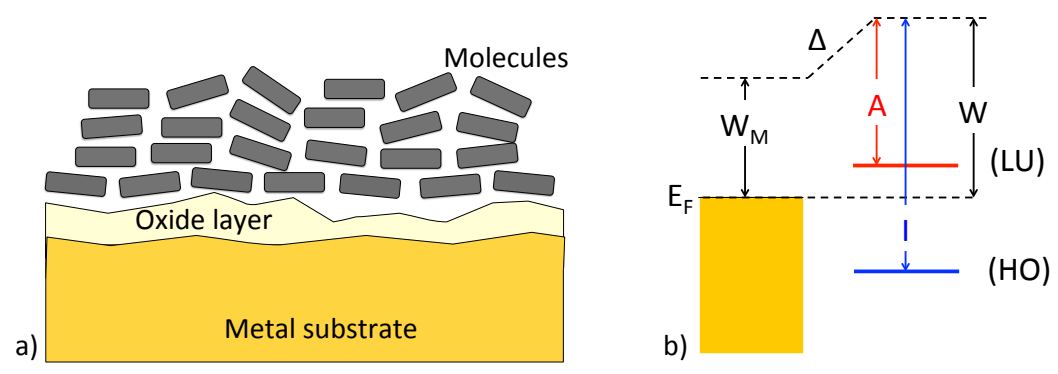

Figure 1.2: a) Sketch of a metal|organic interface structure. b) Model of the energy levels of the same structure. The work function $W_{\mathrm{M}}$ of a clean metal is changed by the formation of a potential step $\Delta$, that results from the interaction with a molecule that has an ionization potential I and an electron affinity A.

diagram for this model system is shown in Figure $1.2 \mathrm{~b}$ ). The vertical $y$-axis of this plot is the energy axis. The filled rectangle represents the occupied energy states of the metal. The highest occupied energy level is called the Fermi level $E_{\mathrm{F}}$. Electrons in metals have a continuum of energy states they can occupy, while electrons in a confined system like a molecule only have discrete states. The highest occupied $(\mathrm{HO})$ and the lowest unoccupied (LU) molecular states are indicated. The position of these levels can be determined by measuring the cost to extract an electron from the molecule (ionisation potential I) and the energy gained by letting the molecule absorb an extra electron (electron affinity A). The interaction with the molecular layer has caused the clean metal work function to change by an amount $\Delta$. The work function that is measured after the molecules have been deposited is then

$$
W=W_{\mathrm{M}}-\Delta
$$

The central questions in this thesis are: how large is $\Delta$ ? What influences it? What are the mechanisms behind the formation of $\Delta$ ?

Three main mechanisms for the work function modification can be distinguished:

- A dipole moment of the molecule or the chemical bond [2]

- Charge redistribution at the interface [Chapter 6]

- Charge transfer across the interface [Chapter 3]

The three mechanisms are schematically illustrated in Figure 1.3. In Figure 1.3 a) the deposited molecules have an intrinsic electric dipole moment (red arrows) and one end that preferentially binds to the metal surface. This leads to the formation of a layer of dipole moments that all point in the same direction. The electric field 

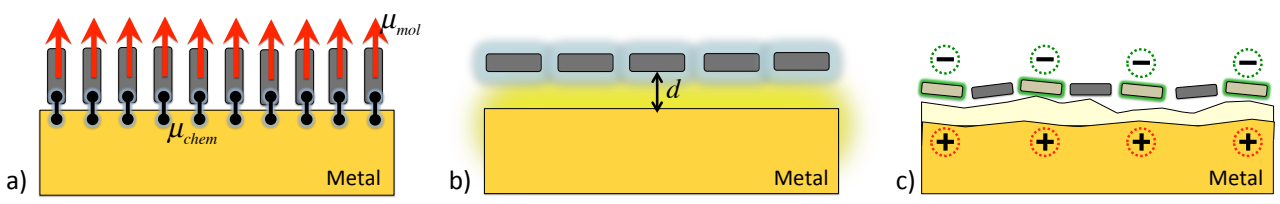

Figure 1.3: Three different mechanisms that can change the work function at a metal|organic interface: a) Molecular and bond dipoles with dipole moments $\mu_{m o l}$ and $\mu_{\text {chem }}, \mathrm{b}$ ) charge redistribution and c) charge transfer.

associated with this dipole layer can either accelerate the electrons out of the metal, effectively reducing the work function or do exactly the opposite, depending on the direction of the arrows. Another possibility is that a molecule with no intrinsic dipole forms a strong bond with the metal and that this bond leads to the formation of a dipole moment[2].

The second mechanism is illustrated in Figure $1.3 \mathrm{~b}$ ), showing a layer of seemingly unperturbed molecules lying flat on the metal surface. With their relatively 'stiff' electron cloud they have deformed the 'soft' electron cloud of the metal surface. A dipole layer is formed due to a 'push back' of electrons into the metal. This effect is also sometimes called, 'the pillow effect', since the deformation of the electron cloud of the metal resembles the effect a heavy object has on a pillow. In chapter 6 this effect is studied in detail. It is found that the Pauli exchange repulsion plays an important role here.

Figure $1.3 \mathrm{c}$ ) shows charged molecules on the metal surface. Some of the molecules have extracted one electron from the metal surface, leaving behind a positively charged hole in the metal. Chapter 3 shows that the number of electrons that are transferred across the interface is determined by a balance between the electrostatic properties of the molecules (electron affinity, ionisation potential and the charging energetics) and the work function of the metal surface. This mechanism can explain why some types of molecules change the work function of a metal to a fixed value for a wide range of metal surfaces with different work functions. The Fermi level of the substrate is fixed to or "pinned" to a molecular level, therefore this effect is referred to as Fermi level pinning. Here it does not matter whether the metal surface is completely clean, the only parameter entering the model is the effective work function of the substrate.

These three mechanisms are not unique for metal|molecule interfaces but apply to any situation where the structure is not changed in an essential way by chemical interactions. In many cases a combination of the mechanisms is at play, for example when acceptor ${ }^{\ddagger}$ molecules are deposited on a clean metal surface. In this case there can be a combination of charge transfer and a charge redistribution at the interface.

\footnotetext{
${ }^{\ddagger}$ Acceptor molecules have a high electron affinity and like to extract electrons from their environment.
} 


\subsection{Novel 2D materials}

Since the first experimental observation of graphene in 2004, interest in 2D materials has boomed. These materials are not 2-dimensional in the mathematical sense, but no material can be made any thinner than this. A graphene flake prepared by the scotch tape method ${ }^{\S}$, can be one carbon atom thick in the $z$-direction while the $x y$ plane consists of many millions of atoms. The prospect of graphene based electronic devices, which would be planar and and atomically thin, has forced scientists to look for suitable substrates and dielectrics that can be used in combination with graphene. Below I would like to highlight two candidates that are compatible with graphene and are explored in this work. First I will introduce the semi-metal graphene itself, then its insulating analogue hexagonal boron-nitride and the semiconductor molybdenum disulfide.

\subsubsection{Graphene}

Graphite consists of layers of graphene weakly bound to each other by van der Waals forces (named after the Dutch physicist and noble laureate Johannes Diderik van der Waals). A graphene sheet consists of carbon atoms bound together in a honeycomb lattice, see Figure 1.4 (top left). It is only one atomic layer thick and stable even in large flakes. Even though the experimental isolation of a single graphene layer was made in recent years, much theoretical effort had already been devoted to studying it. In 1947 the remarkable electronic structure of graphene was calculated by P.R. Wallace[3], who showed that the dispersion relation around the Fermi level is linear,

$$
E(\mathbf{k})= \pm \hbar v_{\mathrm{F}}|\mathbf{k}| .
$$

It is shaped like a cone when the energy is plotted in the $z$-direction as a function of the components of crystal momentum in the $x y$-plane. This is fundamentally different from the dispersion relation in simple metals or semiconductors where the dispersion relation is parabolic, $E=\hbar^{2} k^{2} / 2 m$, with corresponding Fermi velocity $v_{\mathrm{F}}=\frac{1}{\hbar} \frac{\partial E}{\partial k}=\sqrt{2 E / m}$. In this way the velocity of the electrons participating in the conduction depends on the energy. Interestingly, in graphene the Fermi velocity is a constant and does not depend on the energy. Apparently these electrons have zero rest mass and can not be accelerated. However, because of the constant Fermi velocity they do not have to be accelerated to be transported. The Fermi velocity is approximately $1 / 300$ of the speed of light or $10^{6} \mathrm{~m} / \mathrm{s}$. To change the velocity of these electrons, the zero mass must be made finite. This can be done by making the dispersion parabolic, i.e. by opening a band gap[4]. The dispersion relation calculated by the Density Functional Theory method is shown in Figure 1.4 (right).

\footnotetext{
$\S$ Graphene was first isolated by a low tech method; peeling a single graphene layer from graphite using a piece of scotch tape.

"The dispersion refers to the relationship between the energy of the electron and its "crystal" momentum.
} 


$$
\mathrm{y} \underset{\mathrm{x}}{\longrightarrow}
$$

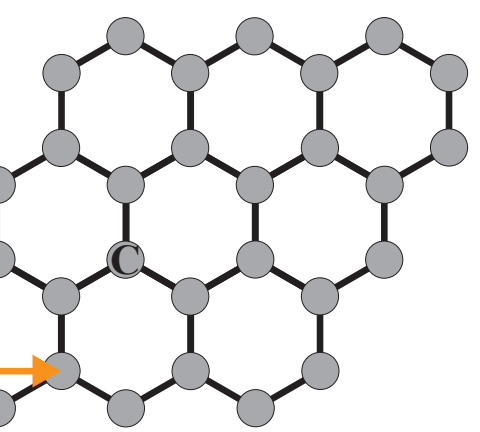

(top)

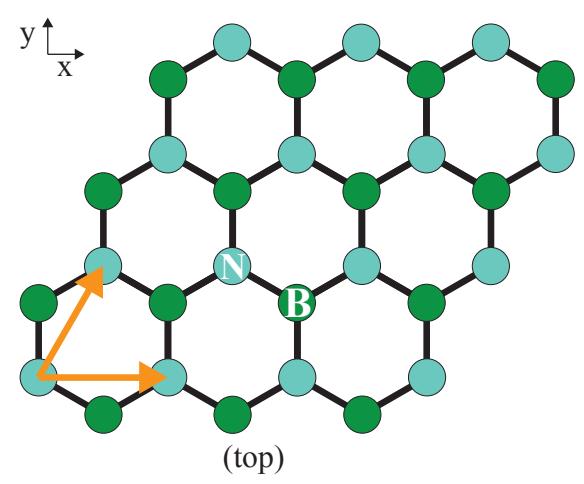

$\mathrm{y} \underset{\mathrm{z}}{\longrightarrow}$

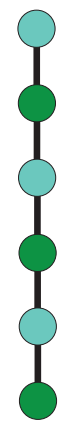

(side)

(side)
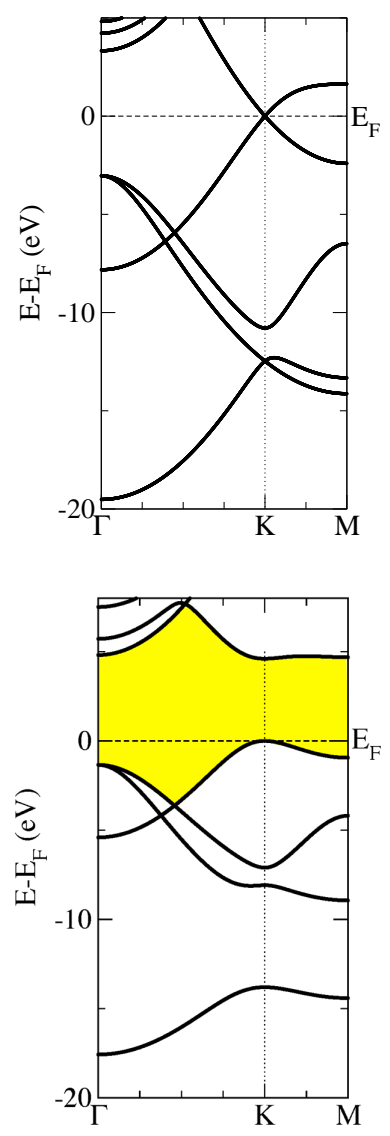

$\underset{\mathrm{x}}{\stackrel{4}{\longrightarrow}}$

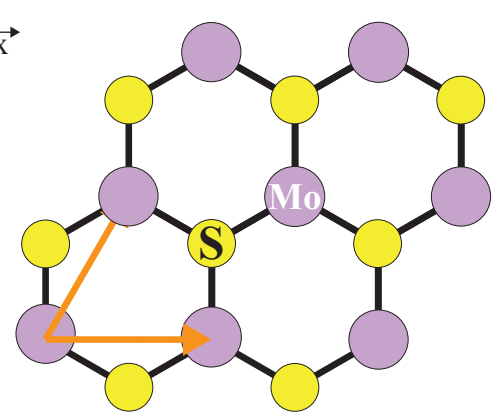

(top)

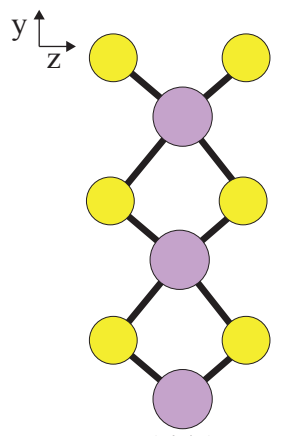

(side)

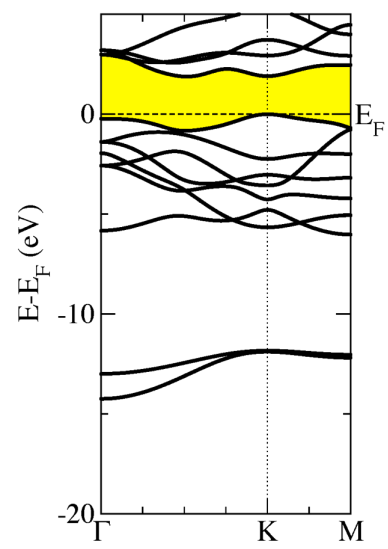

Figure 1.4: From top to bottom: Graphene, hexagonal Boron-Nitride and Molybdenum disulfide. Left: Honeycomb structure seen from the top and a cross section (side) of a single monolayer. Right: Band structure of one monolayer along the high symmetry directions. The band gap is indicated in yellow. 
These kinds of graphs that resemble spaghetti are "band structure" plots. They show the dispersion relation along the high symmetry directions of the crystal. One can see that the bands are linear close to the Fermi level $\left(E_{F}\right)$. They form two cones with a degenerate Fermi level at the K-point.

The property that caused the most widespread attention to this zero gap material is its large room-temperature carrier mobility $\left(10000 \mathrm{~cm}^{2} \mathrm{~V}^{-1} \mathrm{~s}^{-1}\right) \|$, ten times higher than in silicon[1]. If it is possible to build a transistor of graphene, this property will then be very important. Because of the large mobility of the charge carriers, a graphene transistor would be able to support high currents, operate at high frequencies and would have low energy consumption. The only concern is the width of the band gap in graphene. One way to make a band gap in graphene is by placing it on a substrate that breaks the symmetry of the two carbon atoms in the graphene unit cell. A potential candidate that could do this is $h$-BN and is therefore discussed below.

\subsubsection{Hexagonal Boron Nitride}

Hexagonal Boron Nitride $(h-\mathrm{BN})$ is the insulating analog of graphene. While graphene is a semimetal with zero band gap, $h$-BN is a large band gap $(\sim 6 \mathrm{eV})$ insulator. This can be seen in the band structure of $h-\mathrm{BN}$, where the band gap is indicated by the yellow region in Figure 1.4 (right). It consists of Boron an Nitrogen atoms bound in a honeycomb lattice with almost the same lattice dimensions as that of graphene. An atomically flat monolayer can be cleaved from a $h$-BN crystal, because the layers are only weakly coupled. The resemblance between $h$ - $\mathrm{BN}$ and graphene is not coincidental. In the periodic table Carbon (atomic weight 6) can be found in-between Boron (5) and Nitrogen (7). This causes both of their unit cells to have the same amount of electrons and have the same type of bonds. ${ }^{* *}$ Sometimes $h$-BN is referred to as white graphene, because of its large band gap it can not absorb any color in the visible range. Therefore it is transparent in its single crystal form. However, in powder form there are so many small particles with surfaces oriented in all directions that the light is scattered and it appears as white. The electronic structure of graphene makes it possible for light of any color to excite an electron to a higher energy state. This happens only for a fraction of the incoming photons and therefore a single graphene layer is transparent. Graphite consists of many graphene layers and each layer absorbs light. This makes graphite black, both in single crystal and in powder from.

\footnotetext{
$\|$ This is the mobility found in the first experiments, recent measurements with cleaner graphene devices show a mobility that is even an order of magnitude larger.

** Both graphene and $h$-BN get their honeycomb structure because they form so called $s p 2$ hybridised bonds.
} 


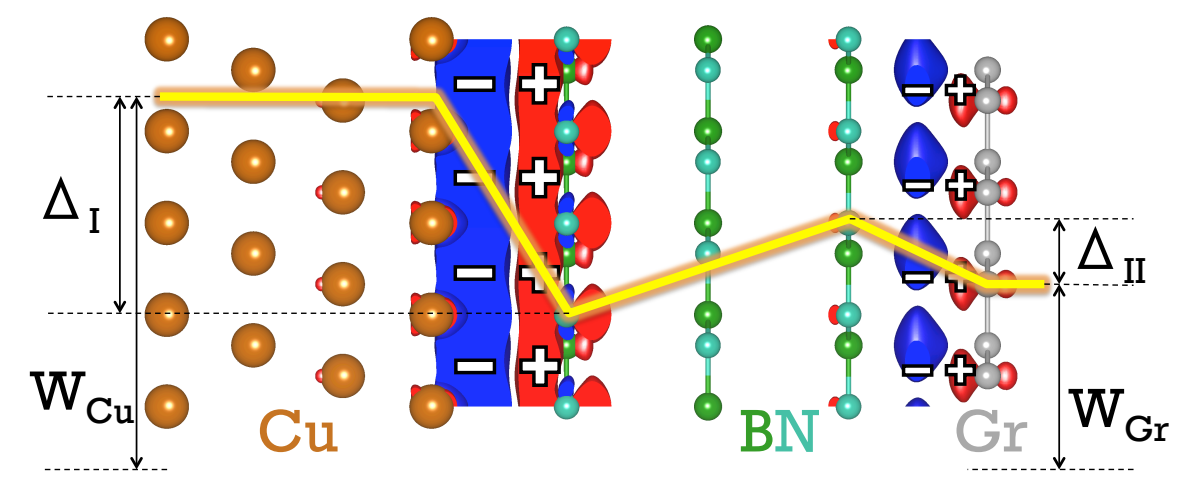

Figure 1.5: Interface dipole layers (blue/red) and the electrostatic potential (yellow) of the nanoscale multilayer structures of Figure 1.1. Each dipole layer causes a step of size $\Delta$ in the potential. An electric field is build up over the $h$-BN layers, as can be seen by the slope of the potential, by the transfer of electrons from copper to graphene.

\subsubsection{Molybdenum disulfide}

Molybdenum disulfide $\left(\mathrm{MoS}_{2}\right)$ is an insulator like $h$ - $\mathrm{BN}$, but its band gap is much smaller, see Figure 1.4 (right) The size of the band gap is such that $\mathrm{MoS}_{2}$ is labeled as a semi-conductor. In-plane $\mathrm{MoS}_{2}$ has a honeycomb lattice like graphene and $h$ $\mathrm{BN}$, but out of plane it is different. The layer of Molybdenum atoms is sandwiched between two layers of Sulfur atoms. Also $\mathrm{MoS}_{2}$ single layers can be cleaved from its larger crystal structure, because the layers are only weakly coupled. In 2011 the first $\mathrm{MoS}_{2}$ based transistor was made and shown to have much better switching capability than graphene.[5] However, the mobility of the charge carriers in $\mathrm{MoS}_{2}$ is low, which makes fast switching very difficult.

\subsection{Charge equilibration across multiple layers}

In multilayer structures like Figure 1.1 there are several interfaces, all of which can have an interface dipole layer with some potential step $\Delta$ as introduced in section 1.2. In chapter 5 the charge transfer in a metal|h-BN|graphene heterostructure is studied and explained in full detail. Here I would like to sketch this mechanism in short.

Figure 1.5 shows a structure with two interfaces, and at both interfaces a dipole layer is formed. The structure consists of two metallic layers, copper and graphene, separated by three insulating layers of $h$-BN. Because the work functions of graphene and copper are unequal, it is energetically favourable for electrons to transfer from one side to the other. This process continues until an energetic balance is obtained 
between the energetic cost of charging the system and the work function difference. At this point you might wonder how electrons can be transferred at all, since there are layers of the insulator $h$-BN in between. This is one of the differences between the macroscopic and the quantum world. For instance electricity cables are insulated by plastic. The insulating layer is thick enough to stop all current from going through it. However, this is not the case for three atomic layers of $h$-BN. In the quantum world electrons can 'tunnel' through barriers like $h$-BN. This means that there is a chance of finding an electron at the copper side that was, a certain time before, at the graphene side. The chance of finding an electron at the other side of the barrier decays exponentially to zero with the thickness of the barrier. This is the reason why you do not get electrocuted when toughing an electric cable.

Figure 1.5 also shows that the dipole layers are confined to the interface, therefore these layers can also be seen as modifiers of the copper and graphene work functions. The direction and amount of charge transfer then depends on the difference between the effective work function of either side. Since the effective work function of the copper is lower then that of graphene $\left(W_{\mathrm{Cu}}-\Delta_{\mathrm{I}}<W_{\mathrm{Gr}}+\Delta_{\mathrm{II}}\right)$, electrons are transferred from copper to graphene. Because of the low density of states in graphene, the transfer of electrons leads to a shift of the Fermi level and it becomes $n$-type doped.

The direction and amount of charge transfer can be changed by applying an external electric field over the structure. In chapters $4 \& 5$ the relation between the applied field strength and the resulting doping level is explained by a planar capacitor model. Surprisingly, this model from classical electrostatics designed for the macro scale, also works here. 


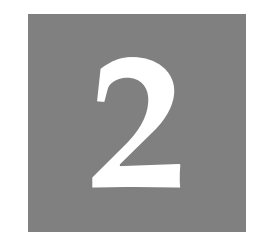

\section{Methods}

In this chapter the techniques behind the electronic structure calculations and the approximations that are needed to make the many body problem tractable are discussed.

\subsection{Density Functional Theory}

The electronic structure calculations in this thesis are based on the density functional theory (DFT) as proposed by P. Hohenberg and W. Kohn in 1964 [6] and W. Kohn and L. J. Sham in 1965 [7]. This approach is a suitable method for calculation of materials properties from first-principles. It is applicable to all kinds of electronic systems such as atoms, molecules, solids and the interfaces between them. Within DFT the electronic density distribution $n(\mathbf{r})$ is the central object rather than the many body wave function $\Psi$. Hohenberg and Kohn showed that knowledge of the ground state density is sufficient to calculate all ground state properties of interest.

Suppose we have a system of $N$ electrons and $M$ ions, then the time-independent Schrödinger equation is

$$
\hat{H} \Psi\left(\mathbf{r}_{1}, \mathbf{r}_{2}, \ldots, \mathbf{r}_{N} ; \mathbf{R}_{1}, \mathbf{R}_{2}, \ldots, \mathbf{R}_{M}\right)=E \Psi\left(\mathbf{r}_{1}, \mathbf{r}_{2}, \ldots, \mathbf{r}_{N} ; \mathbf{R}_{1}, \mathbf{R}_{2}, \ldots, \mathbf{R}_{M}\right),
$$

where $\mathbf{r}_{i}$ and $\mathbf{R}_{i}$ are the coordinates (and spins) of respectively the electrons and ions, and $\hat{H}$ is the Hamiltonian operator acting on the entire interacting system of electrons with mass $m$ and charge $-e$ and ions with mass $M_{j}$ and charge $Z_{j} e$,

$$
\hat{H}=-\frac{\hbar^{2}}{2 m} \sum_{i=1}^{N} \nabla_{i}^{2}-\sum_{i, j} \frac{Z_{j} e^{2}}{\left|\mathbf{r}_{i}-\mathbf{R}_{j}\right|}+\frac{1}{2} \sum_{i \neq j} \frac{e^{2}}{\left|\mathbf{r}_{i}-\mathbf{r}_{j}\right|}-\frac{\hbar^{2}}{2} \sum_{j=1}^{M} \frac{\nabla_{j}^{2}}{M_{j}}+\frac{1}{2} \sum_{i \neq j} \frac{Z_{i} Z_{j} e^{2}}{\left|\mathbf{R}_{i}-\mathbf{R}_{j}\right|}
$$

Since we are only interested in the electronic properties of the system, the problem 
can be simplified by making use of the Born-Oppenheimer approximation [8]. The idea in a nutshell is that the motion of the ions is much slower than that of the electrons, because ions are far heavier than electrons. This makes it possible to factorize the many body wave function into a product of the electronic, $\Phi$ and ionic wave function $\Theta$. The next step is to fix the ions and solve the electronic Schrödinger equation which now depends parametrically on $\mathbf{R}_{j}$ and only acts on the electron wave functions

$$
\begin{gathered}
\hat{H}_{e} \Phi\left(\mathbf{r}_{1}, \mathbf{r}_{2}, \ldots, \mathbf{r}_{N}\right)=E \Phi\left(\mathbf{r}_{1}, \mathbf{r}_{2}, \ldots, \mathbf{r}_{N}\right) \\
\hat{H}_{e}=-\frac{\hbar^{2}}{2 m} \sum_{i} \nabla_{i}^{2}-\sum_{i, j} \frac{Z_{j} e^{2}}{\left|\mathbf{r}_{i}-\mathbf{R}_{j}\right|}+\frac{1}{2} \sum_{i, j} \frac{e^{2}}{\left|\mathbf{r}_{i}-\mathbf{r}_{j}\right|} .
\end{gathered}
$$

Three contributions in the electronic Hamiltonian Eq. (2.4) can be identified. From left to right, the kinetic energy, electron-ion and electron-electron electrostatic interactions. The electron density for this system is defined as

$$
n(\mathbf{r})=<\Phi|\hat{n}(\mathbf{r})| \Phi>
$$

where $\hat{n}(\mathbf{r})$ is the electron density operator

$$
\hat{n}(\mathbf{r})=\sum_{i=1}^{N} \delta\left(\mathbf{r}-\mathbf{r}_{i}\right) .
$$

In 1964 P. Hohenberg and W. Kohn proposed their theory inspired by the ThomasFermi method in which the electron density plays a major role. The foundation of DFT is formulated by two theorems [6]:

- The potential of the nuclei and any external fields working on a system of electrons that feel their mutual Coulomb repulsion is a unique functional of the electronic density $n_{0}(\mathbf{r})$ in the ground state except for a constant shift.

- A universal energy functional $E[n]$ can be defined, which is valid for any number of particles and any external potential. The energy functional has its minimum $E_{0}$ at the ground state density $n_{0}(\mathbf{r})$

$$
E_{0}=E\left[n_{0}(\mathbf{r})\right]=<\Phi_{0}(\mathbf{r})\left|\hat{H}_{e}\right| \Phi_{0}\left(\mathbf{r}^{\prime}\right)>,
$$

which has a useful variational property, $E\left[n_{0}(\mathbf{r})\right] \leq E\left[n^{\prime}(\mathbf{r})\right]$ for any $n^{\prime}(\mathbf{r})$.

Although the density functional theory of Hohenberg and Khon is exact for the ground state properties of any electronic system, it did not provide means for practical calculations. Kohn and Sham changed that in 1965 by using the variational properties of DFT to derive single particle Schrödinger equations. They constructed a non-interacting system with the same ground state density as the original interact- 
ing system and used this to derive the independent particle equations

$$
\left(-\frac{\hbar^{2}}{2 m} \nabla^{2}+v_{\text {eff }}\right) \phi_{n}^{\mathrm{KS}}(\mathbf{r})=\epsilon_{n}^{\mathrm{KS}} \phi_{n}^{\mathrm{KS}}(\mathbf{r}),
$$

with the effective potential

$$
v_{\mathrm{eff}}=v_{\mathrm{ext}}+e^{2} \int \frac{n(\mathbf{r})}{\left|\mathbf{r}-\mathbf{r}^{\prime}\right|} d \mathbf{r}+\frac{\delta E_{\mathrm{xc}}[n]}{\delta n(\mathbf{r})} .
$$

Here $E_{\mathrm{xc}}[n]$ is a functional that includes exchange and correlation interactions between the electrons. The sum over the squares of the single particle wave functions gives the electron density:

$$
n(\mathbf{r})=\sum_{n}^{N}\left|\phi_{n}^{\mathrm{KS}}\right|^{2}
$$

To get results out of this approach equations (2.8)-(2.10) have to be solved self- consistently. This means choosing some trial vectors $\left\{\phi_{n}^{\mathrm{KS}}\right\}_{n}^{N}$, calculating $n(\mathbf{r})$, operating with $\hat{H}_{e}$, creating a new set $\left\{\phi_{n}^{\prime}{ }^{\prime K S}\right\}_{n}^{N}$ and calculating the new $n(\mathbf{r})$. This cycle is repeated until convergence has been achieved.

The accuracy of the self-consistently calculated ground state density $n_{0}$ and the corresponding energy $E_{0}$ is in principle only limited by the exchange-correlation (XC) functional $E_{\mathrm{xc}}$ used in the calculation. If the exact functional were known, this approach would yield the exact ground state of the many body problem. Since the exact functional is not known, approximate functionals have been proposed and are still being developed today. See section 2.4 for the different types of XC functionals that have been used in this thesis work.

\subsection{Plane wave pseudopotential method}

In the previous section the Kohn-Sham independent single particle equations,

$$
\left(-\frac{\hbar^{2}}{2 m} \nabla^{2}+v_{\mathrm{eff}}\right) \phi_{n}(\mathbf{r})=\epsilon_{n} \phi_{n}(\mathbf{r}),
$$

were discussed. In order to solve this equation one needs to define a basis set in which $\phi_{n}(\mathbf{r})$ can be expressed. Plane waves form an orthonormal basis set which can be made more complete by adding plane waves with shorter wave lengths. The orthonormality of the plane wave basis means that

$$
\frac{1}{\Omega} \int_{\Omega} e^{-i \mathbf{q}^{\prime} \cdot \mathbf{r}} e^{i \mathbf{q} \cdot \mathbf{r}} d \mathbf{r}=\delta_{\mathbf{q}^{\prime}, \mathbf{q}}=\left\langle\mathbf{q}^{\prime}\right| \mathbf{q}>,
$$

where $\Omega$ is the volume of space of one period and $\mathbf{q}$ is the wave vector. The Fourier theorem says that any continuous periodic function can be expressed in a Fourier series. Our interest lies in periodic structures, either crystal structures or structures 
within a supercell, resulting in a periodic $v_{\text {eff }}$. The Bloch theorem then states that 2 the eigenstates of the one electron Hamiltonian can be chosen to have the form of a plane wave times a function with the periodicity of the lattice

$$
\phi_{n \mathbf{k}}(\mathbf{r})=e^{i \mathbf{k} \cdot \mathbf{r}} u_{n \mathbf{k}}(\mathbf{r}),
$$

where $u_{n \mathbf{k}}(\mathbf{r}+\mathbf{R})=u_{n \mathbf{k}}(\mathbf{r})$ for all $R$ in the lattice. Therefore plane waves are a straightforward basis for the wave function $u_{n \mathbf{k}}(\mathbf{r})$

$$
u_{n \mathbf{k}}(\mathbf{r})=\frac{1}{\sqrt{\Omega}} \sum_{m} c_{\mathbf{G} n \mathbf{k}} e^{i \mathbf{G}_{m} \cdot \mathbf{r}},
$$

where $\mathbf{G}_{m}$ a reciprocal lattice vector and $c_{\mathbf{G} n \mathbf{k}}$ are the Fourier coefficients. The Schrödinger equation can then be written for any $\mathbf{k}$ vector as the following matrix equation:

$$
\sum_{m} H_{\mathbf{q}, \mathbf{q}^{\prime}}(\mathbf{k}) c_{n, \mathbf{q}^{\prime}}=\epsilon_{n}(\mathbf{k}) c_{n, \mathbf{q}}
$$

where $\mathbf{q}=\mathbf{k}+\mathbf{G}_{m}, \mathbf{q}^{\prime}=\mathbf{k}+\mathbf{G}_{m^{\prime}}$ and

$$
H_{\mathbf{q}, \mathbf{q}^{\prime}}(\mathbf{k}) c_{n, \mathbf{q}^{\prime}}=\left\langle\mathbf{k}+\mathbf{G}_{m}\left|\hat{H}_{\mathrm{KS}}\right| \mathbf{k}+\mathbf{G}_{m^{\prime}}\right\rangle=\frac{\hbar^{2}}{2 m}\left|\mathbf{k}+\mathbf{G}_{m}\right|^{2} \delta_{m, m^{\prime}}+v_{\mathrm{eff}}\left(\mathbf{G}_{m}-\mathbf{G}_{m^{\prime}}\right)
$$

and $\mathbf{G}_{m^{\prime}}$ is the reciprocal lattice vector which differs from $\mathbf{G}_{m^{\prime}}$ by $\mathbf{G}_{m^{\prime \prime}}=\mathbf{G}_{m}-\mathbf{G}_{m^{\prime}}$ [9]. The Bloch theorem shows that all possible eigenstates are given by the wave vectors $\mathbf{k}$ within a primitive cell. The most compact primitive cell is the first Brillouin zone and it can be found by drawing the Wigner-Seitz cell in reciprocal space.

In practice, a basis set of $3 N_{\text {pw }}$ plane waves with energies up to $\frac{\hbar^{2}}{2 m}|\mathbf{k}+\mathbf{G}|^{2}<$ $E_{\text {cut }}$ is defined. As a consequence the number of plane waves in the basis of the eigenfunctions can vary depending on $\mathbf{k}$. Calculating the kinetic energy contributions in the Hamiltonian eq. (2.16) 'costs' $N_{\text {pw }}$ operations, since $\left.<\mathbf{k}+\mathbf{G}\left|\nabla^{2}\right| \phi\right\rangle=$ $\frac{\hbar^{2}(\mathbf{G}+\mathbf{k})^{2}}{2 m} c_{n, \mathbf{q}}$ is diagonal in reciprocal space and is therefore a fast operation. A problem arises when one tries to calculate $\left\langle\mathbf{k}+\mathbf{G}\left|v_{\text {eff }}\right| \phi>\right.$ in reciprocal space; this requires evaluating a double sum, resulting in expensive $N_{\mathrm{pw}}^{2}$ calculations. The solution is based on the fact that evaluating $\left\langle\mathbf{k}+\mathbf{G}\left|v_{\text {eff }}\right| \phi>\right.$ in real space requires only the number of real space grid points $N_{\mathrm{R}}$ operations. If we would have a mechanism to switch from reciprocal to real space and back in less than $N_{\mathrm{pw}}^{2}$ calculations, then it is favorable to go to real space and calculate the effective potential contributions to the Hamiltonian there. Such a mechanism exists and it is called the Fast Fourier Transform (FFT). Using the FFT it is possible to go to real space in $N_{\mathrm{R}} \log N_{\mathrm{R}}$ calculations. A drawback is that $N_{\mathrm{R}}$ in practice has to be an order of magnitude larger than $N_{\mathrm{pw}}$ to keep the same accuracy. The charge density is the square of the wave function, therefore the periodicity is doubled and double the Fourier components are needed in each direction. Once in real space, one just calculates $v_{\text {eff }}(\mathbf{r}) \phi(\mathbf{r})$ point by point and after that uses the FFT to switch to reciprocal space. 


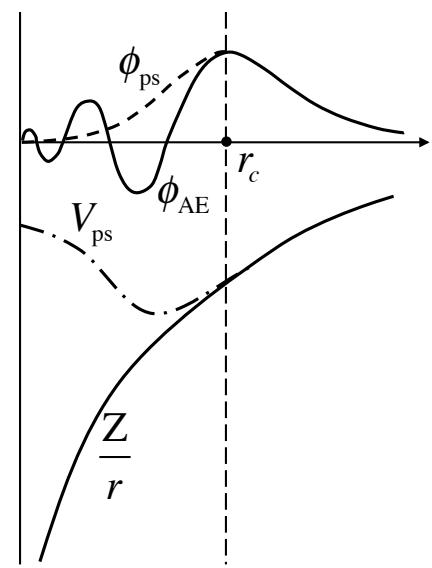

Figure 2.1: Schematic representation of the all-electron potential $\left(\frac{Z}{r}\right)$ and the pseudopotential $\left(V_{\mathrm{ps}}\right)$ and the corresponding wave functions $\left(\phi_{\mathrm{ps}} \& \phi_{\mathrm{AE}}\right)$. The all-electron and pseudoelectron wave functions/potentials match at $r=r_{c}$. Figure is a redraw from a figure in Ref. [10].

The plane wave basis has the major advantage, that the kinetic energy operator is exactly diagonalized. The biggest problem for a plane wave basis is to account for the rapidly varying behaviour of the wave function near the nucleus, see Fig. 2.1. Describing this behaviour accurately would require an enormous number of plane waves. Electrons in these states are bound strongly to the nucleus and do not contribute to the formation of bonds or to conduction. The solution is to use a smooth pseudopotential instead of the singular Coulomb potential. The main idea behind the pseudopotential is that the core electrons do not participate in chemical bonding an therefore do not change when the bonding changes. The pseudopotential method forces the lowest orbitals to always stay occupied. These electrons 'shield' the valence electrons against the Coulomb potential. Pseudopotentials are then the smoothed potentials in which the valence electrons move.

To better understand the pseudopotential method we discuss the norm-conserving pseudopotentials constructed for atoms. In 1979 Hamann, Schlüter and Chiang proposed a method to produce pseudopotentials obeying the following conditions [11]:

- Real and pseudo valence eigenvalues are the same for a chosen atomic reference configuration.

- Real and pseudo atomic wave functions are identical beyond a chosen core radius $r_{c}$.

- The norms of the real and pseudo wave functions for $r \leq r_{c}$ must be equal 
(norm conservation condition)

$$
\int_{0}^{r_{c}}|\phi(r)|^{2} 4 \pi r^{2} d r=\int_{0}^{r_{c}}\left|\phi^{\mathrm{ps}}(r)\right|^{2} 4 \pi r^{2} d r
$$

- The logarithmic derivatives of the real and pseudo wave function and their first energy derivative agree at $r_{c}$.

$$
D_{l}\left(\varepsilon, r_{c}\right)=\left.r \frac{\phi_{l}^{\prime}(\varepsilon, r)}{\phi_{l}(\varepsilon, r)}\right|_{r_{c}}=\left.D_{l}^{\mathrm{ps}}\left(\varepsilon, r_{c}\right) \frac{\partial D_{l}(\varepsilon, r)}{\partial \varepsilon}\right|_{r_{c}}=\left.\frac{\partial D_{l}^{\mathrm{ps}}(\varepsilon, r)}{\partial \varepsilon}\right|_{r_{c}}
$$

The norm conservation condition ensures that the total charge within the core region is conserved. Producing a norm-conserving pseudopotential for an atom involves three basic steps. Firstly one has to make an all-electron (AE) calculation by integration of the radial Schrödinger equation. Secondly, at a chosen reference energy $\varepsilon_{l}^{\mathrm{AE}}$ with corresponding $\phi_{l}^{\mathrm{AE}}$, find some $\phi_{l}^{\mathrm{ps}}$, which is nodeless, has the same logarithmic derivative at $r_{c}$ as $\phi_{l}^{\mathrm{AE}}$ and obeys the norm-conservation condition. When such a wave function is found, one can use the radial Schrödinger equation to calculate the pseudopotential $V_{\mathrm{ps}}$ :

$$
-\frac{\phi_{\mathrm{ps}}^{\prime \prime}}{2}+\left(\frac{(l+1) l}{2 r^{2}}+V_{\mathrm{ps}}-\varepsilon_{l}^{\mathrm{AE}}\right) \phi_{\mathrm{ps}}=0
$$

This pseudopotential is not unique for an atom, one can change the size of the core radius and create hard or soft pseudopotentials. Soft pseudopotentials have a large core radius, resulting in a smoother potential, which reduces the number of plane waves needed for the pseudo wave functions. However this comes at the cost of less accurate description of the wave function close to the atom. The calculations in this work are performed with so called non-conserving ultra-soft pseudopotentials.[12] One of advantages of using non-conserving pseudopotentials instead of norm-conserving pseudopotentials is that they are not bound to the norm conservation condition and therefore require a smaller set of plane waves, reducing the computational cost. The solution for the difference in the norm between the pseudo and AE wave functions was solved by D. Vanderbilt by adding augmentation charges.[13] These charges are constructed in such a way that the correct multipole moment of the AE charge distribution is regained. In this method the core radius can be as large as half the nearest neighbour distance, resulting in soft pseudo potentials.

\subsection{Projector Augmented Wave method}

In 1994 P.E. Blöchl proposed a method [14] that merges an all electron (AE) method and a pseudopotential method. This is the method used in the electronic structure program VASP[12] that is used in this thesis. In this method space is divided into re- 


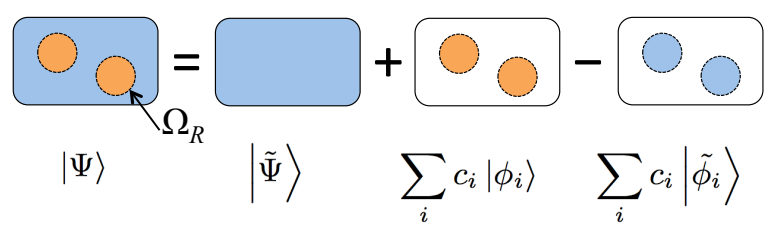

Figure 2.2: Schematic representation of the Projector Augmented Wave Method.

gions close to the nuclei and interstitial regions, see Figure 2.2. The electronic wave functions have strong oscillations close to the cores while they vary smoothly in the interstitial regions. Blöchl proposed to solve for a set of pseudo (PS) wave functions on a lattice of pseudo potentials and augment the rapidly oscillating core part onto it.

Suppose there is some linear transformation $\mathcal{T}$ which after operating on the PS wave function gives you the $\mathrm{AE}$ wave function, i.e. $|\Psi\rangle=\mathcal{T}|\tilde{\Psi}\rangle$. Assuming that in the interstitial region $|\tilde{\Psi}\rangle=|\Psi\rangle, \mathcal{T}$ can be split up into a sum of local projections,

$$
\mathcal{T}=1+\sum_{R} \mathcal{T}_{R}
$$

where $R$ are the coordinates of the cores. The operator $\mathcal{T}_{R}$ works in a spherical region $\Omega_{R}$ around each core site and has to be made for each atom type. Each PS wave function within $\Omega_{R}$ can be expanded into a set of PS partial waves,

$$
\left|\tilde{\Psi}_{n}\right\rangle=\sum_{i} c_{i, n}\left|\tilde{\phi}_{i}\right\rangle,
$$

where $n$ has been introduced as the band index. Also $\left|\Psi_{n}\right\rangle$ can be expanded into (another) set of partial waves, which means that for a proper operator $\mathcal{T}$

$$
\left|\Psi_{n}\right\rangle=\sum_{i} c_{i, n}\left|\phi_{i}\right\rangle=\mathcal{T} \sum_{i} c_{i, n}\left|\tilde{\phi}_{i}\right\rangle
$$

within $\Omega_{R}$. The coefficients $c_{i, n}$ are the same for both sets of partial waves, and are calculated by a scalar product of 'projector functions' $p_{i}$ with the PS wave function,

$$
c_{i, n}=\left\langle p_{i} \mid \tilde{\Psi}_{n}\right\rangle .
$$

The projector functions are localized in $\Omega_{R}$ and should satisfy the condition $\left\langle p_{i} \mid \phi_{j}\right\rangle=$ $\delta_{i, j}$. The coefficients $c_{i, n}$ are in practice calculated on a radial support grid. The PS wave functions are mapped onto this grid and the coefficients $c_{i, n}$ are acquired by a 
point by point multiplication. The total $\mathrm{AE}$ wave function can be written as,

$$
\left|\Psi_{n}\right\rangle=\left|\tilde{\Psi}_{n}\right\rangle+\sum_{i} c_{i, n}\left|\phi_{i}\right\rangle-\sum_{i} c_{i, n}\left|\tilde{\phi}_{i}\right\rangle .
$$

where the PS wave function is augmented in the second term and the PS solution in $\Omega_{R}$ is cut out in the third term. The linear transformation defined by Blöchl is then given by

$$
\mathcal{T}=1+\sum_{R}\left|\phi_{i}\right\rangle-\left|\tilde{\phi}_{i}\right\rangle\left\langle p_{i}\right| .
$$

The total energy in the PAW formalism can be expressed in a global and two on-site terms. For example the kinetic energy

$$
E_{\mathrm{kin}}=\sum_{n} f_{n}\left\langle\Psi_{n}\left|\frac{\nabla^{2}}{2}\right| \Psi_{n}\right\rangle
$$

can in the PAW be expressed formalism as

$E_{\text {kin }}=\sum_{n} f_{n}\left\langle\tilde{\Psi}_{n}\left|\frac{\nabla^{2}}{2}\right| \tilde{\Psi}_{n}\right\rangle+\sum_{R} \sum_{i, j} \rho_{i, j}\left\langle\phi_{i}\left|\frac{\nabla^{2}}{2}\right| \phi_{j}\right\rangle-\sum_{R} \sum_{i, j} \rho_{i, j}\left\langle\tilde{\phi}_{i}\left|\frac{\nabla^{2}}{2}\right| \tilde{\phi}_{j}\right\rangle$,

a sum of three terms $E_{\text {kin }}=\tilde{E}+E^{1}-\tilde{E}^{1}$.

Also the electronic charge density in the PAW is split up into a global and on-site terms, three different charge densities are calculated in real space as

$$
\begin{gathered}
\tilde{n}(\mathbf{r})=\sum_{n} f_{n}\left\langle\tilde{\Psi}_{n} \mid \mathbf{r}\right\rangle\left\langle\mathbf{r} \mid \tilde{\Psi}_{n}\right\rangle \\
n^{1}(\mathbf{r})=\sum_{i, j} \rho_{i, j}\left\langle\phi_{i} \mid \mathbf{r}\right\rangle\left\langle\mathbf{r} \mid \phi_{j}\right\rangle \\
\tilde{n}^{1}(\mathbf{r})=\sum_{i, j} \rho_{i, j}\left\langle\tilde{\phi}_{i} \mid \mathbf{r}\right\rangle\left\langle\mathbf{r} \mid \tilde{\phi}_{j}\right\rangle,
\end{gathered}
$$

with $\rho_{i, j}=\sum_{n} f_{n} c_{i, n}^{*} c_{j, n}$ the on-site density matrix and $n=\tilde{n}+n^{1}-\tilde{n}^{1}$ the AE electronic charge density. For calculation of the Hartree energy the total charge density,

$$
n_{T}=n+n_{Z c}=\left(\tilde{n}+\hat{n}+n_{Z} \tilde{Z}_{c}\right)+\left(n^{1}+n_{Z c}\right)-\left(\tilde{n}^{1}+\hat{n}+n_{Z} c\right)=\tilde{n}_{T}+n_{T}^{1}-\tilde{n}_{T}^{1}
$$

is needed. Here $n_{Z c}=n_{Z}+n_{c}$, the charge density of the nuclei and the AE charge density which is frozen in by construction of the pseudopotential. Because the PS wave functions do not have the same norm as the AE wave functions, a soft compensation charge $\hat{n}$ is added in order to compensate for the long range multipole interactions between the spheres. When done properly the multipole moment of the charge density within the sphere $\left(\tilde{n}^{1}+\hat{n}\right)$ has the same as the multipole moment of 
the AE charge density $\left(n^{1}\right)$.[12] In VASP the Hartree energy is then calculated as

$$
E_{\mathrm{H}}=\frac{1}{2} \int \frac{\tilde{n}_{T}(\mathbf{r}) \tilde{n}_{T}\left(\mathbf{r}^{\prime}\right)}{\left|\mathbf{r}-\mathbf{r}^{\prime}\right|} d \mathbf{r} d \mathbf{r}^{\prime}+\frac{1}{2} \int_{\Omega_{R}} \frac{n_{T}^{1}(\mathbf{r}) n_{T}^{1}\left(\mathbf{r}^{\prime}\right)}{\left|\mathbf{r}-\mathbf{r}^{\prime}\right|} d \mathbf{r} d \mathbf{r}^{\prime}-\frac{1}{2} \int_{\Omega_{R}} \frac{\tilde{n}_{T}^{1}(\mathbf{r}) \tilde{n}_{T}^{1}\left(\mathbf{r}^{\prime}\right)}{\left|\mathbf{r}-\mathbf{r}^{\prime}\right|} d \mathbf{r} d \mathbf{r}^{\prime}
$$

where the first term is an integral over the entire cell and the second an third term are on-site integrals over the spheres. To arrive at this expression the approximation has been made that in one of the cross terms in the Hartree energy of $n_{T}, \tilde{n}_{T}$ is replaced by its on-site approximation $\tilde{n}_{T}^{1}$. If the set of projectors is complete then this is exact. In reality it creates a small error, but comes with the great advantage of having no cross terms between the global plane-wave grid and the local radial grid.

A similar division in on-site and global terms can be made for the exchange energy. In all cases where expectation values are calculated, whether it be calculation of energy contributions or the charge density, the two on-site terms are evaluated on a radial grid and the global term is evaluated on the full plane wave grid.

\subsection{Exchange and Correlation functionals}

\subsubsection{Local Density Approximation \& Generalized-Gradient Ap- proximation}

In section 2.1 the concept of an exchange-correlation (XC) functional $E_{\mathrm{xc}}$ was introduced. The accuracy of the solution of the DFT problem compared to the solution of the full many-body problem depends on the XC functional that is used. The simplest exchange-correlation (XC) functional is the local density approximation (LDA). In this approximation $E_{\mathrm{xc}}[n]$ is approximated locally by the exchange-correlation energy of a homogenous electron gas with the same density:

$$
E_{\mathrm{xc}}^{\mathrm{LDA}}[n]=\int \varepsilon_{\mathrm{xc}}(n(\mathbf{r})) n(\mathbf{r}) d \mathbf{r}
$$

Here $\varepsilon_{x c}$ is the exchange-correlation energy per particle of a homogenous electron gas of density $n$. The exchange and correlation part can be split up as

$$
\begin{gathered}
\epsilon_{x c}=\epsilon_{x}+\epsilon_{c}, \\
\epsilon_{x}(n) \equiv-\frac{1}{2}\left(\frac{9 \pi}{4}\right)^{1 / 3} \frac{1}{r_{s}},
\end{gathered}
$$

with $(4 \pi / 3) r_{s}^{3}=n^{-1}$ the radius of a sphere containing one electron. The exchange part in known analytically but the correlation part has to be estimated. A very accurate calculation was made by Ceperley and Adler in 1980 [15], a parametrization [16] of their data is also used in this thesis. Inclusion of the spin degree of freedom is done by substitution $\varepsilon_{\mathrm{xc}}(n(\mathbf{r}))$ of Eq. (2.32) by $\varepsilon_{\mathrm{xc}}\left(n_{\uparrow}(\mathbf{r}), n_{\downarrow}(\mathbf{r})\right)$, where the exchangecorrelation energy is now a function of the local spin up/down charge densities. The 
resulting functional is known as the local spin density approximation (LSDA).

Even though the LDA seems like a crude approximation, calculations with LDA exchange-correlation have provided quite accurate results for many applications. The LDA gives ionization energies of atoms, dissociation energies of molecules and cohesive energies with a fair accuracy of typically $10-20 \%$ [17]. When predicting band gaps in semiconductors one has to be careful, because the LDA is known to underestimate the size of the gap [18]. However, the LDA gives bond lengths and the geometries of molecules and solids typically with a remarkable accuracy of $1 \%$ [17].

A more elaborate $\mathrm{XC}$ (semi-local) functional takes into account the spatially varying density within the system. The generalized-gradient approximation (GGA) is one of the most commonly used functionals which takes into account this inhomogeneity. It does this by including the local derivative of the charge density into the functional,

$$
E_{\mathrm{xc}}^{\mathrm{GGA}}\left[n_{\uparrow}, n_{\downarrow}\right]=\int f\left(n_{\uparrow}(\mathbf{r}), n_{\downarrow}(\mathbf{r}), \nabla n_{\uparrow}(\mathbf{r}), \nabla n_{\downarrow}(\mathbf{r})\right) d \mathbf{r} .
$$

There are many different types of GGA functionals, all have a slightly different function $f$. The GGA's of Perdew and Wang (PW91)[19] and Perdew, Burke and Enzerhof (PBE) [20] are among the most used in the field.

\subsubsection{Beyond local functionals}

A problem with the $\mathrm{XC}$ functionals mentioned in the previous paragraph is that they are semi-local functionals. The exchange and correlation energies are calculated point by point including only information of the charge density $(n(\mathbf{r}), \nabla n(\mathbf{r}))$ at that point in space. While for instance the exact Fock exchange energy,

$$
E_{x}=-\frac{e^{2}}{2} \sum_{\mathbf{k} n, \mathbf{q} m} f_{\mathbf{k} n} f_{\mathbf{q} m} \times \int \frac{\phi_{\mathbf{k} n}^{*}\left(\mathbf{r}^{\prime}\right) \phi_{\mathbf{q} m}^{*}\left(\mathbf{r}^{\prime}\right) \phi_{\mathbf{k} n}^{*}(\mathbf{r}) \phi_{\mathbf{q} m}^{*}(\mathbf{r})}{\left|\mathbf{r}-\mathbf{r}^{\prime}\right|} d \mathbf{r} d \mathbf{r}^{\prime},
$$

is by definition a nonlocal object. In the Hartree-Fock (HF) method this exchange energy is calculated exactly. A serious problem in HF is its the description of metals. The Fermi velocity $\left(\left.\frac{d \epsilon}{d k}\right|_{k_{F}}\right)$ has a singularity at $k_{F}$, resulting in an undefined Fermi velocity, while in experiment well-defined velocities can be measured. This is not a problem for systems with a large band gap between the valence and conduction band, such as insulators and molecules. Therefore this method is often used in quantum chemistry.

In an attempt to improve the description of molecular energetics and band gaps, a class of XC functionals has been developed that mix in a percentage of exact exchange with semi-local exchange and correlation energy. These functionals are known as Hybrid Functionals. Since HF gives band gaps that are too large and LDA gives too small gaps, the hope is that a combination of these methods results in better 
agreement with experiment. Remember that this is no longer DFT, since inclusion of an exchange term like Eq. (2.34) (which is not a density functional) makes it an orbital method. One can make all kind of flavours containing $x \%$ Fock exchange and $(100-x) \%$ LDA/GGA exchange. These kind of functionals then have to be benchmarked to a reference database. One widely used hybrid functionals is the B3LYP functional, that combines $80 \%$ LDA and $20 \%$ HF exchange. However, like HF it fails in its description of metals.[21]

One of the problems faced in this thesis is accounting for the weak van de Waals forces between 2D layered materials in DFT. For instance GGA functionals give no binding between the layers of a hexagonal boron-nitride crystal and HF gives purely repulsive binding curves. While the LDA does seem to give a reasonable equilibrium binding distance, the binding energy is underestimated and the binding energy versus binding distance curve has the wrong asymptotic behavior for large distances. An asymptotic behavior of $d^{-4}$ is expected for van der Waals interacting planes.[22] However an exponential decay is found, because the decay is determined by the overlap of the wave functions which decays exponentially. An attempt by M.Dion et al. to solve this issue without calculation of costly terms is to use a van der Waals Density Functional $E_{\mathrm{vdW}}[n]=E_{x}[n]+E_{c}[n]$.[23] The correlation energy in this functional is split up in two parts

$$
E_{c}[n]=E_{c}^{\mathrm{LDA}}[n]+E_{c}^{\mathrm{nl}}[n]
$$

with a part LDA correlation that describes the short-range interaction and a nonlocal part for the long-range interaction. $E_{c}^{\mathrm{nl}}[n]$ has the form of a six dimensional integral

$$
E_{c}^{\mathrm{nl}}[n]=\frac{1}{2} \iint n(\mathbf{r}) \Phi\left(\mathbf{r}, \mathbf{r}^{\prime}\right) n\left(\mathbf{r}^{\prime}\right) d \mathbf{r} d \mathbf{r}^{\prime}
$$

where $\Phi\left(\mathbf{r}, \mathbf{r}^{\prime}\right)=\Phi\left(d, d^{\prime}\right)$ is the so called van der Waals kernel. This kernel is a general function that depends on two distances: $d=\left|\mathbf{r}-\mathbf{r}^{\prime}\right| q_{0}(\mathbf{r})$ and $d^{\prime}=\left|\mathbf{r}-\mathbf{r}^{\prime}\right| q_{0}\left(\mathbf{r}^{\prime}\right)$, where $q_{0}$ is a pre factor which describes a modulation of the local Fermi vector. The kernel can be tabulated in advance and is universal. For large $d$ this asymptotic form is

$$
\Phi \rightarrow-\frac{C}{d^{2} d^{\prime 2}\left(d^{2}+d^{\prime 2}\right)},
$$

resulting in a $\frac{C}{R^{6}}$ interaction between two points. The exchange energy can be chosen from any (semi-)local exchange functional. Which works best for a particular system should be determined by benchmarking to experimental data. 


\subsection{The $\mathrm{G}_{0} \mathrm{~W}_{0}$ approximation}

In the previous section the Fock exchange was introduced and with it a view into orbital based methods. The GW method is also an orbital based method and it gives a more accurate description of the excitation spectra. For instance, in the LDA the band gaps of semiconductor and insulators are underestimated. A GW correction of the band gap gives most of the time a much better agreement with the experimental band gap. In the GW method[24] the self energy $\Sigma=i G W$, the contribution to the bare particle energy by its interaction with the system, is approximated as

$$
\Sigma\left(\mathbf{r}, \mathbf{r}^{\prime}, E\right)=\frac{i}{2 \pi} \int_{-\infty}^{\infty} e^{-i \omega^{\prime} \delta} G\left(\mathbf{r}, \mathbf{r}^{\prime}, E-\omega\right) W\left(\mathbf{r}, \mathbf{r}^{\prime}, \omega\right) d \omega
$$

where $G$ is the Green's function and $W=\epsilon^{-1} V_{\mathrm{C}}$ is the screened Coulomb interaction $V_{\mathrm{C}}$. A positive infinitesimal number $\delta$ assures that the contour integral is closed just above the real axis and that it encompasses only poles corresponding to occupied states. The dielectric matrix $\epsilon^{-1}$ is calculated with the random phase approximation.[25] The GW quasi particle (QP) equations that have to be solved look like

$$
\left(-\frac{\hbar^{2}}{2 m} \nabla^{2}+v_{\mathrm{ext}}+e^{2} \int \frac{n(\mathbf{r})}{\left|\mathbf{r}-\mathbf{r}^{\prime}\right|} d \mathbf{r}\right) \psi_{n \mathbf{k}}(\mathbf{r})+\int \Sigma\left(\mathbf{r}, \mathbf{r}^{\prime}, E_{n \mathbf{k}}\right) \psi_{n \mathbf{k}}\left(\mathbf{r}^{\prime}\right) d \mathbf{r}^{\prime}=E_{n \mathbf{k}} \psi_{n \mathbf{k}}(\mathbf{r}) .
$$

The first term on the left side of Eq. (2.39) is the same as in the Kohn-Sham equations of DFT, see Eqs. (2.8)\&(2.9). However in the second term the XC potential is replaced by the GW self energy. The resulting quasi particle energies are given by [18]

$$
E_{n \mathbf{k}}=\left\langle\psi_{n \mathbf{k}}(\mathbf{r})\left|E_{\text {kin }}+v_{e x t}+V_{\mathrm{H}}+\Sigma\left(E_{n \mathbf{k}}\right)\right| \psi_{n \mathbf{k}}(\mathbf{r})\right\rangle .
$$

Since the right hand side already requires the QP eigenvalues, $E_{n \mathbf{k}}$ has to be solved iteratively. In this thesis we use only one iteration step. This process is called $G_{0} W_{0}$ or 'single shot' GW and results in a modification of the Kohn-Sham eigenvalues $\epsilon_{n \mathbf{k}}$

$$
E_{n \mathbf{k}}=\epsilon_{n \mathbf{k}}+Z_{n \mathbf{k}} \operatorname{Re}\left[\left\langle\psi_{\mathrm{nk}}\left|\mathrm{E}_{\mathrm{kin}}+\mathrm{v}_{\mathrm{ext}}+\mathrm{V}_{\mathrm{H}}+\Sigma\left(\epsilon_{\mathrm{nk}}\right)\right| \psi_{\mathrm{nk}}\right\rangle-\epsilon_{\mathrm{nk}}\right],
$$

with renormalisation factor $Z_{n \mathbf{k}}=\left(1-\left\langle\psi_{n \mathbf{k}}\left|\frac{\partial \Sigma(\omega)}{\partial \omega}\right|_{\epsilon_{n \mathbf{k}}} \mid \psi_{n \mathbf{k}}\right\rangle\right)^{-1}$ and $\psi_{n \mathbf{k}}$ the KohnSham orbitals form the DFT calculation.[26] Evaluation of the elements $\left\langle\psi_{n \mathbf{k}}|\Sigma(\omega)| \psi_{n \mathbf{k}}\right\rangle$ scales with $N_{\mathrm{b}} \times N_{\omega} \times N_{\mathrm{k}}^{2} \times N_{\mathrm{pw}}^{2}$, the number of bands, frequencies, k-points and plane waves, respectively. This makes the GW calculations much more costly compared to DFT, see section 2.2. 


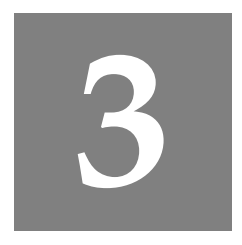

\section{Fermi level pinning by integer charge transfer at electrode-organic semiconductor interfaces*}

The atomic structure of interfaces between conducting electrodes and molecular organic materials varies considereably. Yet experiments show that pinning of the Fermi level, which is observed at such interfaces, does not depend upon the structural details. In this paper we develop a general model to explain Fermi level pinning, and formulate simple expressions for the pinning levels, based upon integer charge transfer between the conductor and the molecular layer. In particular we show that DFT calculations give good values for the pinning levels.

\subsection{Introduction}

Interfaces play an important role in organic light emitting diodes and solar cells. The Schottky barriers at the interfaces between conducting electrodes and organic semiconductors determine the injection and collection of charge carriers.[27, 28, 29] In organic molecular semiconductors the molecules preserve their identity as they interact via relatively weak intermolecular (van der Waals) forces. Charge localization and polaron effects then play a much more important role than in conventional wide band semiconductors.[30, 31] Experimentally the formation of Schottky barriers at electrode-organic interfaces is dominated by the first molecular layer. Often these barriers are characterized by monitoring the work function of an organic layer deposited onto a metallic substrate through ultraviolet photoelectron spectroscopy

\footnotetext{
*Based on: M. Bokdam, D. Cakir and G. Brocks, Fermi level pinning by integer charge transfer at electrode-organic semiconductor interfaces, Applied Physics Letters 98, 113303 (2011).
} 
(UPS). Such experiments in combination with first-principles calculations have lead to an impressive progress in understanding the interaction between well-ordered layers of organic molecules and single crystal metal surfaces, as well as to simple models for the resulting work functions.[32, 33, 34, 35, 36, 37, 38, 39, 40]

The contacts between electrodes and organic materials in actual devices are likely to be less well-defined than in these model systems, however. Metal electrodes are often covered by an (amorphous) native oxide layer and conducting oxides or polymers, such as ITO or PEDOT, are commonly amorphous. UPS experiments on interfaces between such electrodes and organic materials show nevertheless a universal behavior. For substrates with work functions within a certain energy interval the organic layer changes the work function depending on the details of the interaction between molecules and substrate (such as the pillow or pushback effect). Remarkably, outside this interval the work function is completely pinned, i. e. independent of the substrate work function, see Fig. 3.1.[29] All substrates with a work function lower than $W^{-}$lead to pinning at $W^{-}$, and all substrates with a work function higher than $W^{+}$lead to pinning at $W^{+}$. The pinning levels seem to be related to the electron affinity $(A)$ and the ionization potential $(I)$ of the molecules in the layer, respectively. However, measured values of $W^{-/+}$differ from $A$ or $I$ by up to $\sim 1 \mathrm{eV}$. Such "shifts" have been ascribed to polaron effects, but seem far too large to originate from molecular relaxation only.

\subsection{Integer charging model}

Here we develop and test a model for Fermi level pinning at electrode-organic semiconductor interfaces. We will argue that pinning occurs by transfer of electrons to or from the molecular levels, and that the shifts observed experimentally mainly originate from the electrostatic interactions at the interface, which lower the energy. For an arbitrary charge distribution this would lead to a shift that depends on the amount of charge transfer, but if the interfacial charge distribution can be described by a plane capacitor, the shift is constant. The lower and upper pinning levels $W^{-/+}$, see Fig. 3.1 are then given by

$$
W^{-}=A+B^{-}, W^{+}=I-B^{+},
$$

where $B^{-/+}$is the Coulomb energy associated with charging a molecule in the interface layer with an electron/hole. The individual parameters $A, B, I$ depend on the environment of the molecule, as charged molecules are screened by their environment. As is discussed below, such screening terms cancel in $W^{-/+}$, which implies that the Fermi level is pinned and that the values of the pinning levels are a property of the molecular layer only. For example, for C60 layers adsorbed on different low work function substrates a pinning level is observed at $W^{-}=4.5 \mathrm{eV}$.[41]. The difference between this value and the electron affinity $A=4.0 \mathrm{eV}$ found for C60 layers, is in good agreement with the charging energy $B^{-}=0.55 \pm 0.15$ of a C60 molecule 

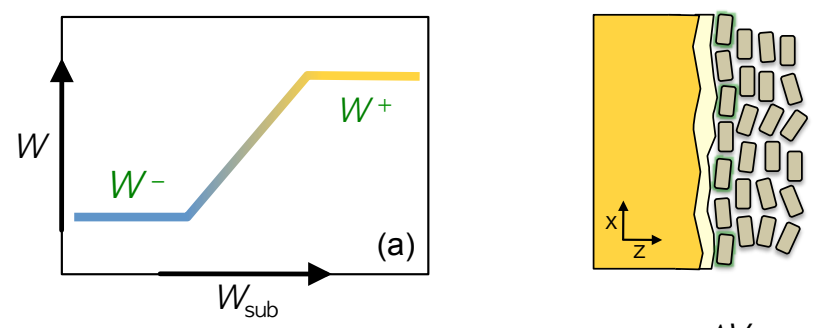

(b)
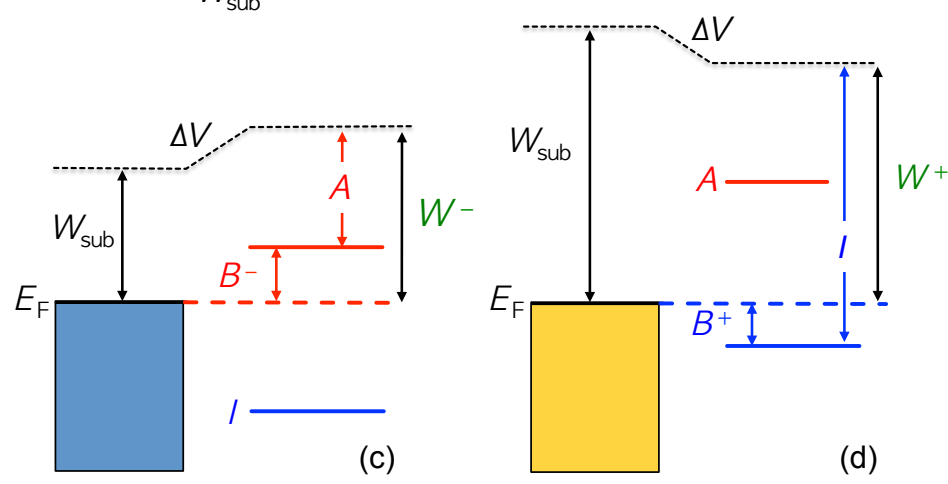

Figure 3.1: (a) The work function $W$ of the electrode-organic interface (b) as func-

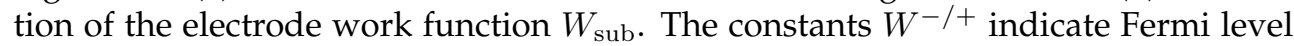
pinning on low/high work function substrates, according to level diagrams (c)/(d), respectively.

in a layer.[42]

In principle the parameters $A, B, I$ can be extracted from experiment or from calculations. For density functional theory (DFT) calculations with standard generalized gradient approximation (GGA) functionals additional simplifications are possible, yielding very simple approximations for the pinning levels

$$
W^{-}=-\epsilon_{\mathrm{LUMO}}+E_{\mathrm{rel}}^{-} ; W^{+}=-\epsilon_{\mathrm{HOMO}}-E_{\mathrm{rel}}^{+},
$$

where $\epsilon_{\mathrm{HOMO}} / \mathrm{LUMO}$ are the DFT (Kohn-Sham) energy levels of the highest occupied/lowest unoccupied molecular orbitals (HOMO/LUMO) of the neutral molecular layer. $E_{\text {rel }}^{-/+}$are the energies associated with structural relaxation of the molecule upon charging it with an electron or hole, or in other words, the energies attributed to polaron effects. The latter turn out to be relatively small for the molecules we have tested.

Pinning can only take place if a potential step $\Delta V$ occurs at the interface, see Fig. 3.1. If there is no significant hybridization between the substrate and the molecular wave functions, a potential step must be the result of transfer of an integer number of electrons between the substrate and the molecular layer. We derive a simple model for such integer charge transfer (ICT) of electrons from the substrate to a layer of acceptor molecules, resulting in an expression for $W^{-}$.[29] Deriving an expression 
for $W^{+}$, which involves electron transfer from donor molecules to the substrate, then proceeds along the same lines.

The work function of a substrate covered by a molecular layer is given by

$$
W=-E_{\mathrm{F}}+\Delta V\left(N_{1}\right),
$$

where $E_{\mathrm{F}}$ is the Fermi level of the metal ( $-E_{\mathrm{F}}$ is the work function of the substrate without the molecular layer). $\Delta V\left(N_{1}\right)$ is the potential step caused by the $N_{1}$ charges in the molecular layer and the screening charges in the substrate. The details of the charge distribution do not matter for the work function, as the latter is measured far from the interface, compared to the molecular dimensions.[43] One can express the potential step in terms of a dipole density

$$
\Delta V\left(N_{1}\right)=\frac{e D}{\varepsilon_{0} a} \frac{N_{1}}{N}
$$

with $D$ is a dipole associated with each charged molecule and its image charge, $a$ the surface area covered by one neutral or charged molecule, and $N$ the total number of molecules. Defining an interface capacitance by $C=e \varepsilon_{0} a N / D$ allows one to rewrite $\Delta V$ in plane capacitor form

$$
\Delta V\left(N_{1}\right)=\frac{N_{1} e^{2}}{C}
$$

The total energy energy of a layer of $N_{1}$ charged molecules and $N-N_{1}$ neutral molecules is

$$
E_{\text {tot }}\left(N_{1}\right)=\left(N-N_{1}\right) E_{0}+N_{1} E_{1}+E_{\mathrm{C}}\left(N_{1}\right),
$$

where $E_{0}$ and $E_{1}$ are the total energies of a neutral and a charged molecule, respectively. $E_{\mathrm{C}}\left(N_{1}\right)$ is the electrostatic Coulomb energy of the interfacial arrangement of the charged molecules, all polarization and image charge effects included. If we assume that the elecrostatic energy of the interface is described well by a plane capacitor with a capacitance as in Eq. (3.5), then

$$
E_{\mathrm{C}}\left(N_{1}\right)=\frac{N_{1}^{2} e^{2}}{2 C}-N_{1} B^{-} .
$$

Here $B^{-}$is the Coulomb energy associated with charging a single molecule. It has to be subtracted from the plane capacitor charging energy to avoid double counting, as per definition it is already accounted for in $E_{1}$, see Eq. (3.6). The electro-chemical potential of the ensemble of molecules (at $T=0)$ is given by $\mu=E_{\text {tot }}\left(N_{1}+1\right)-$ $E_{\text {tot }}\left(N_{1}\right)$ in the thermodynamic limit $N_{1} \gg 1$. At equilibrium $\mu$ is equal to $E_{\mathrm{F}}$, the Fermi level of the substrate, which fixes the number of charged molecules $N_{1}$. Using this in Eqs. (3.5) and (3.3), and the definition of the electron affinity $A=E_{0}-E_{1}$, then leads to $W^{-}$as in Eq. (3.1); $W^{+}$is derived in a similar way.

Note that the Fermi level (or work function) of the substrate, as well as the capacitance $C$ of the interface, drop out of the expression. One might argue that Eq. (3.1) 
still does not represent work function pinning, as all individual parameters $A, I$ and $B^{-/+}$strongly depend on the environment of the molecule, including the substrate. Screening by an environment stabilizes a charged molecule, thereby increasing its electron affinity and decreasing its ionization potential. Replacing a substrate alters the screening energy by $\Delta E_{\mathrm{S}}$ and changes the electron affinity and ionization potential to $A^{\prime}=A+\Delta E_{\mathrm{S}}$ and $I^{\prime}=I-\Delta E_{\mathrm{S}}$, respectively. However, at the same time the charging energy of a molecule is changed to $B^{\prime}=B-\Delta E_{\mathrm{S}}$, which shows that the values of $W^{-/+}$obtained from Eq. (3.1) are independent of the substrate. In other words, we have work function pinning and the pinning levels are a property of the molecular layer only.

\subsection{DFT parametrisation}

The parameters $A, I$ and $B^{-/+}$are accessible from (first-principles) calculations on isolated molecular layers. ${ }^{\dagger}$ In particular for DFT calculations with GGA or LDA functionals further simplifying approximations are possible. Those functionals yield an expression of the molecular total energy that is analytical in the occupation numbers $n_{i}$ of the Kohn-Sham energy levels $\varepsilon_{i}$. Janak's theorem holds for such functionals, which expresses the energy levels as derivatives of the total energy $\varepsilon_{i}=$ $\partial E_{\mathrm{mol}} / \partial n_{i}$. The vertical electron affinity can then be approximated by

$$
A_{\mathrm{v}}=-\int_{0}^{1} \varepsilon_{\mathrm{LUMO}}(n) d n \approx-\epsilon_{\mathrm{LUMO}}-\frac{1}{2} U,
$$

assuming that a linear approximation $\varepsilon_{\mathrm{LUMO}}(n)=\epsilon_{\mathrm{LUMO}}+U n$ is sufficiently accurate. Here $\epsilon_{\mathrm{LUMO}}$ is the Kohn-Sham LUMO level of a neutral molecule in the molecular layer. Integrating Janak's expression in the same approximation gives $E_{\text {mol }}(n)=E_{0}+\epsilon_{\mathrm{LUMO}} n+\frac{1}{2} U n^{2}$ for the total energy of a molecule with a partially occupied LUMO level. The quadratic term can be associated with the molecular charging energy

$$
B^{-} \approx \frac{1}{2} U
$$

Eq. (3.1) requires the adiabatic $A$ and $I$, which can be obtained from the corresponding vertical properties by correcting with the molecular relaxation energies, i.e. $A=A_{\mathrm{v}}+E_{\text {rel }}^{-}$and $I=I_{\mathrm{v}}+E_{\text {rel }}^{+}$. Using Eqs. (3.8) and (3.9) then gives Eq. (3.2).

We calculate Kohn-Sham energy levels using the Vienna Ab initio Simulation Package (VASP) with Projector Augmented Waves (PAWs) and the PW91 generalized gradient approximation (GGA) functional.[44, 45, 12] Calculations using the PBE functional yield pinning levels that are within $0.05 \mathrm{eV}$ of the values presented in this paper. The calculations are performed for close-packed molecular monolayers. The unit cell in the direction perpendicular to that plane is chosen sufficiently

\footnotetext{
${ }^{\dagger}$ The fact that screening does not affect the pinning level does not mean that it is a purely molecular property, however. Static electrostatic molecular multipoles can affect the energy levels of a molecule in a layer, see Ref. [35].
} 
Table 3.1: Calculated relaxation energies, pinning levels according to Eq. (3.2), and experimental pinning levels; all in eV. Pinning is to the LUMO, i.e. $W^{-}$, unless indicated otherwise.

\begin{tabular}{lccc}
\hline molecule & $E_{\text {relax }}$ & $W$ calc. & $W$ exp. \\
\hline F4-TCNQ & 0.10 & 5.7 & $5.6^{b}$ \\
TCNQ & 0.10 & 5.4 & $4.8^{c}$ \\
PTCDA & 0.09 & 4.7 & $4.7^{d}$ \\
C60 & 0.05 & 4.4 & $4.5^{e}$ \\
CuPc & 0.05 & 3.3 & $3.3^{f}$ \\
CuPc $^{a}$ & 0.02 & 4.4 & $4.4^{g}$ \\
$\mathrm{TTF}^{a}$ & 0.10 & 4.2 & $4.2^{c}$ \\
\hline
\end{tabular}

${ }^{a}$ pinning to the HOMO, i.e. $W^{+},{ }^{b}$ Ref. [46], ${ }^{c}$ Ref. [47], ${ }^{d}$ Ref. [48], ${ }^{e}$ Ref. [41], ${ }^{f}$ Ref. [49], ${ }^{g}$ Ref. [50].

large that the potential in the middle of the cell represents the vacuum level. The Kohn-Sham energy levels are then calculated with respect to this vacuum level.

\subsection{Conclusion}

The pinning levels calculated according to Eq. (3.2) are given in Table 3.1. The calculated values are in good agreement with available experimental data, indicating that the essential physics is grasped by our simple model. TCNQ seems to be an exception, where the calculation overestimates the value of the pinning level to the LUMO. Note that the relaxation energies play a relatively unimportant role in fixing the pinning levels, as they are quite small. The energy difference between the upper and the lower pinning levels $W^{+}-W^{-}$for CuPc is less than half the band gap in this material.[51] Wereas our model gives a natural explanation for this effect, it would be difficult to explain this on the basis of molecular relaxation only. 


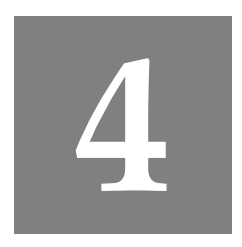

\section{Electrostatic doping of graphene through ultrathin hexagonal boron nitride films*}

When combined with graphene, hexagonal boron nitride $(h-B N)$ is an ideal substrate and gate dielectric with which to build metal|h-BN|graphene field-effect devices. We use firstprinciples density functional theory (DFT) calculations for $C u \mid h$-BN|graphene stacks to study how the graphene doping depends on the thickness of the $h$-BN layer and on a potential difference applied between $\mathrm{Cu}$ and graphene. We develop an analytical model that describes the doping very well, allowing us to identify the key parameters that govern the device behaviour. When the $h-B N$ is very thin, this behaviour is dominated by novel interface terms that we evaluate from DFT calculations for the individual materials and for interfaces between $h-B N$ and $\mathrm{Cu}$ or graphene.

\subsection{Introduction}

The preparation of monolayers of graphite (graphene) has led to exciting discoveries associated with the unique electronic structure of this two-dimensional system.[1, 52] Transport experiments are usually performed in a field-effect device geometry with a graphene flake separated from a gate electrode by a dielectric spacer.[53, 54] Doped $\mathrm{Si}$ is commonly used as the gate material and $\mathrm{SiO}_{2}$ as the dielectric. Substrate inhomogeneities $[55,56]$ and trapped charges $[57,58]$ lead to local variations of the electrostatic potential at the $\mathrm{SiO}_{2}$ surface which results in uncontrolled local electron-

*Based on: M. Bokdam, P.A. Khomyakov, G. Brocks, ZC. Zhong and P.J. Kelly, Electrostatic Doping of Graphene through Ultrathin Hexagonal Boron Nitride Films, Nano Letters 11, 4631-4635 (2011). 
and hole-doping of the graphene sheet, so-called electron-hole puddles.[59, 58]

With a layered graphene-like honeycomb structure and a lattice constant only $1.7 \%$ larger, insulating [60] $h$-BN would be the ideal substrate for graphene based devices;[4] the interaction between $h$-BN and graphene sheets is so weak that even if their lattices are forced to be commensurate, the characteristic electronic structure of graphene is barely altered. For an incommensurate stacking of graphene on $h$ $\mathrm{BN}$, the perturbation is even smaller, leading to an extremely well-ordered graphene layer with a very high carrier mobility.[61, 62, 63]

Like graphene, $h$-BN layers can be prepared by mechanical exfoliation.[64] Cleaved layers can also be thinned to a single layer with a high-energy electron beam.[65] Alternatively, $h$-BN layers can be grown by chemical vapor deposition (CVD) on transition metals such as $\mathrm{Cu}$ or $\mathrm{Ni}$, using precursors such as borazine $\left(\mathrm{B}_{3} \mathrm{~N}_{3} \mathrm{H}_{6}\right)$ or ammonia borane $\left(\mathrm{NH}_{3}-\mathrm{BH}_{3}\right)$. [66, 67] With a proper choice of growth conditions, homogeneous ultrathin $h$-BN only 1-5 atomic layers thick can be grown. Moreover, graphene can be grown by CVD on top of a $h$-BN layer adsorbed on a metal substrate,[68] which is ideal for field-effect devices.

A field effect is created by applying a voltage difference between graphene and the metal substrate, resulting in charge accumulation or depletion in the graphene layer. We study this so-called electrostatic doping as a function of the thickness of the $h$-BN layer and the applied voltage using first-principles (DFT) calculations for a $\mathrm{Cu}(111) \mid h$-BN $\mid$ graphene structure. In the absence of an applied voltage, a spontaneous charge transfer across the $h$-BN layer occurs between the metal substrate and the graphene, leading to an intrinsic doping of graphene. This transfer, which is driven by the difference in graphene and metal work functions, is strongly modified by the charge displacements resulting from the weak chemical interactions at the metal $\mid h$-BN and at the $h$-BN|graphene interfaces.

By varying the applied voltage, the doping level can be controlled. Experimentally the position of the Fermi level in graphene has a square-root like dependence on the voltage.[69, 58, 70,62,63] We will demonstrate that this behavior is reproduced by our DFT calculations on $\mathrm{Cu}(111) \mid h$-BN|graphene and develop an analytical model that describes how the Fermi level depends on the applied voltage and the $h$-BN layer thickness.

\subsection{Computational Details}

We use DFT at the level of the local density approximation (LDA), within the framework of the plane-wave PAW pseudopotential method,[14] as implemented in VASP. $[44,45,12]$ The LDA gives a reasonable description of the geometries resulting from the weak interactions between $h$-BN layers and between a $h$-BN and a graphene layer.[4] It also describes the interaction between graphene and metal (111) surfaces very reasonably.[71, 72, 73] We expect it to provide a description of the interaction between $h$-BN and metal (111) surfaces of similar quality. In contrast, PW91 or PBE GGA functionals (incorrectly) predict essentially no interlayer binding between $h$ - 


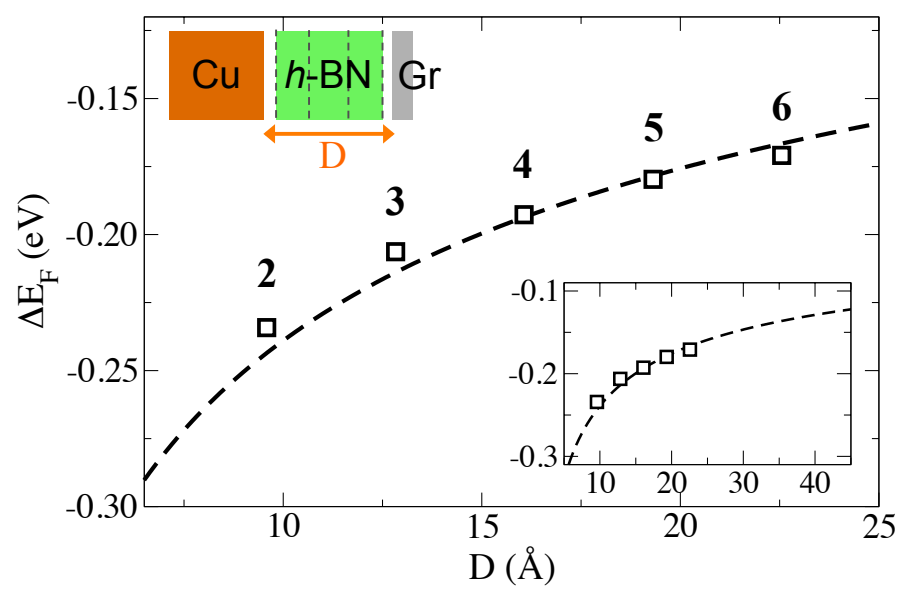

Figure 4.1: The Fermi level shift $\Delta E_{\mathrm{F}}$ in graphene versus the thickness $\mathrm{D}$ of the $h$-BN film. The squares represent DFT results on $\mathrm{Cu}(111) \mid h$-BN $\mid$ graphene structures with 2 - 6 layers of $h$-BN. The dashed line represents the model of Eq. (4.3).

BN or graphene layers, or between metal (111) surfaces and $h$-BN or graphene sheets.

The $\mathrm{Cu}(111) \mid h$-BN|graphene structures are modeled by a periodic slab with six $\mathrm{Cu}$ atomic layers in the (111) surface orientation, a slab of 1-6 layers of $h$-BN, a graphene monolayer, and a vacuum region of $\sim 15 \AA$. A dipole correction is applied to avoid spurious interactions between periodic images of the slab. We choose the lattice constant of graphene equal to its optimized LDA value $a=2.445 \AA$, and scale the in-plane lattice constants of $\mathrm{Cu}(111)$ and $h$-BN so that the structure can be represented in a $1 \times 1$ graphene surface unit cell.[4, 72,73] The positions of the atoms in graphene and $h$-BN, and of the top two atomic layers of the metal surface are allowed to relax during geometry optimization. An electric field applied across the slab is modeled with a sawtooth potential.

In the most stable configuration of a $h$-BN monolayer on $\mathrm{Cu}(111)$, the nitrogen atoms are adsorbed on top of $\mathrm{Cu}(111)$ surface atoms and the boron atoms occupy hollow sites. The calculated equilibrium separation between $h$-BN and the $\mathrm{Cu}$ surface is $3.11 \AA$. The graphene and $h$-BN layers are stacked as in Ref. 4 with a calculated graphene- $h$-BN equilibrium separation of $3.21 \AA$, and a separation between $h$-BN layers of $3.24 \AA$. The equilibrium structures are changed negligibly by the applied electric field, at least for field strengths up to $0.5 \mathrm{~V} / \AA$. Graphene interacts weakly with $h$-BN, which essentially preserves the characteristic linear band structure of graphene about the conical points. The weak interaction induces a small band gap of $\sim 60 \mathrm{meV}$ in graphene, provided the $h$-BN and the graphene lattices are commensurate. If the lattices are incommensurate, the gap seems to disappear.[62, 61, 63] In the following this point will not be important. 


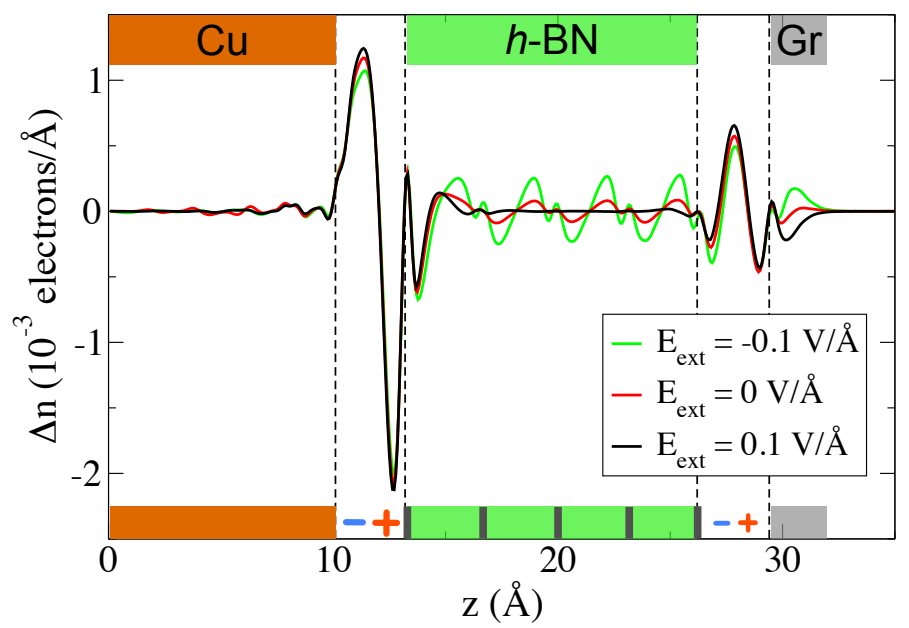

Figure 4.2: Plane-integrated electron density difference $\Delta n(z)$ for a $\mathrm{Cu}(111) \mid h-$ $\mathrm{BN} \mid$ graphene structure with five $h$-BN layers. The positions of the top layer of $\mathrm{Cu}$ atoms, the bottom and top $h$-BN layers, and of the graphene layer are indicated by vertical dashed lines. The three curves give $\Delta n(z)$ for three different values of an external electric field $E_{\text {ext }}$.

\subsection{Intrinsic Doping}

We monitor the doping of graphene in the $\mathrm{Cu}(111) \mid h$-BN $\mid$ graphene structure by calculating the change of the Fermi level $\Delta E_{\mathrm{F}}$ with respect to neutral graphene. A computationally convenient way $[72,73]$ of characterizing $\Delta E_{\mathrm{F}}$ is in terms of the difference between the metal|dielectric|graphene stack and free-standing graphene work functions, $W$ and $W_{\mathrm{G}}$,

$$
\Delta E_{\mathrm{F}}=W-W_{\mathrm{G}},
$$

Negative (positive) values of $\Delta E_{\mathrm{F}}$ then correspond to $n$-type ( $p$-type) doping. Figure 4.1 shows $\Delta E_{\mathrm{F}}$ as a function of the number of $h$-BN layers. These numbers are calculated without an external field which means that in the $\mathrm{Cu}(111) \mid h$-BN $\mid$ graphene structure the graphene sheet is intrinsically doped with electrons. $\Delta E_{\mathrm{F}}$ decreases with the number of $h$-BN layers. We will model this thickness dependence below.

The intrinsic doping originates from electrons that are transferred from the $\mathrm{Cu}$ electrode to the graphene sheet across the $h$-BN layer. However, the calculated work functions of $\mathrm{Cu}(111)$ and graphene are $W_{\mathrm{M}}=5.25 \mathrm{eV}$ and $W_{\mathrm{G}}=4.48 \mathrm{eV}$, respectively. If charge transfer is driven by the difference between the $\mathrm{Cu}$ substrate and graphene work functions alone, then establishing a common Fermi level would require transferring electrons from graphene to $\mathrm{Cu}$ and the result would be $p$-type doping, i.e. a positive value of $\Delta E_{\mathrm{F}}$, at variance with the results shown in Fig. 4.1. The $h$-BN layer must therefore play a non-trivial role.

The interaction between two materials at an interface generally results in the 
formation of an interface dipole. The latter can be visualized in terms of the electron rearrangement at the interface characterized by the electron density of the entire system minus the electron densities of the two separate materials (using identical, frozen atomic structures). As only the dependence perpendicular to the interface is relevant, it is convenient to work with plane-integrated electron densities $n(z)=\iint n(x, y, z) d x d y$ where the integration is over the surface unit cell. The electron displacement in a $\mathrm{Cu}(111) \mid h$-BN|graphene stack is then described by $\Delta n(z)=$ $n_{\mathrm{M}|\mathrm{BN}| \mathrm{Gr}}(z)-n_{\mathrm{M}}(z)-n_{\mathrm{BN}}(z)-n_{\mathrm{Gr}}(z)$. The result for a $\mathrm{Cu}(111)|h-\mathrm{BN}|$ graphene structure with five layers of $h$-BN is shown in Fig. 4.2.

Dipoles are clearly visible at the interfaces between the different materials. The largest interface dipole is between $\mathrm{Cu}$ and $h$-BN. Electrons are piled up on the $\mathrm{Cu}$ surface implying depletion close to the $h$-BN surface. A similar effect is observed in the adsorption of organic molecules on metal surfaces.[74, 75] Between $h$-BN and graphene one observes a similar, but significantly smaller interface dipole. These dipoles are truly localized at the interfaces; their sizes do not depend on the number of $h$-BN layers as long as there is more than one. Both dipoles can also be obtained in separate calculations for the two interfaces, i.e. one for $h$-BN adsorbed on $\mathrm{Cu}(111)$, and one for graphene adsorbed on $h$-BN.

An interface dipole layer results in a discontinuity in the potential energy perpendicular to the interface. The potential energy step at an $\mathrm{A} \mid \mathrm{B}$ interface can be obtained from an $\mathrm{A} \mid \mathrm{B}$ slab calculation as the difference between the work functions on the $\mathrm{A}$ and the $\mathrm{B}$ sides of the slab. In practice, a single $h$-BN layer on top of the $\mathrm{Cu}(111)$ surface is sufficient to calculate the potential energy step $\Delta_{\mathrm{Cu} \mid \mathrm{BN}}$ at the $\mathrm{Cu}(111) \mid h-\mathrm{BN}$ interface. Similarly, the potential energy step $\Delta_{\mathrm{BN} \mid \mathrm{C}}$ at the $h$-BN|graphene interface can be determined with a system comprising two $h$-BN layers and the graphene sheet.

We find $\Delta_{\mathrm{Cu} \mid \mathrm{BN}}=1.12 \mathrm{eV}$, and $\Delta_{\mathrm{BN} \mid \mathrm{C}}=0.14 \mathrm{eV}$. Without additional charge transfer the difference between the Fermi levels in $\mathrm{Cu}$ and graphene in the $\mathrm{Cu}(111) \mid h$ $\mathrm{BN} \mid$ graphene structure would be

$$
V_{0}=W_{\mathrm{M}}-W_{\mathrm{G}}-\Delta_{\mathrm{Cu} \mid \mathrm{BN}}-\Delta_{\mathrm{BN} \mid \mathrm{C}}
$$

which we calculate to be $V_{0}=-0.49 \mathrm{eV}$. To achieve equilibrium requires transferring electrons between $\mathrm{Cu}$ and graphene to set up an electrostatic potential that compensates for $V_{0}$. The sign of $V_{0}$ indicates that electrons are transferred from $\mathrm{Cu}$ to graphene, which results in $n$-type doping, i.e. a negative value for $\Delta E_{\mathrm{F}}$, in agreement with Fig. 4.1.

\subsection{External Field}

The charge transfer leads to an intrinsic electric field across the $h$-BN slab, which polarizes the $h$-BN layers. This polarization is clearly identifiable in the red line in Fig. 4.2 as small oscillations of $\Delta n(z)$ in the $h$-BN region. It can be eliminated by 


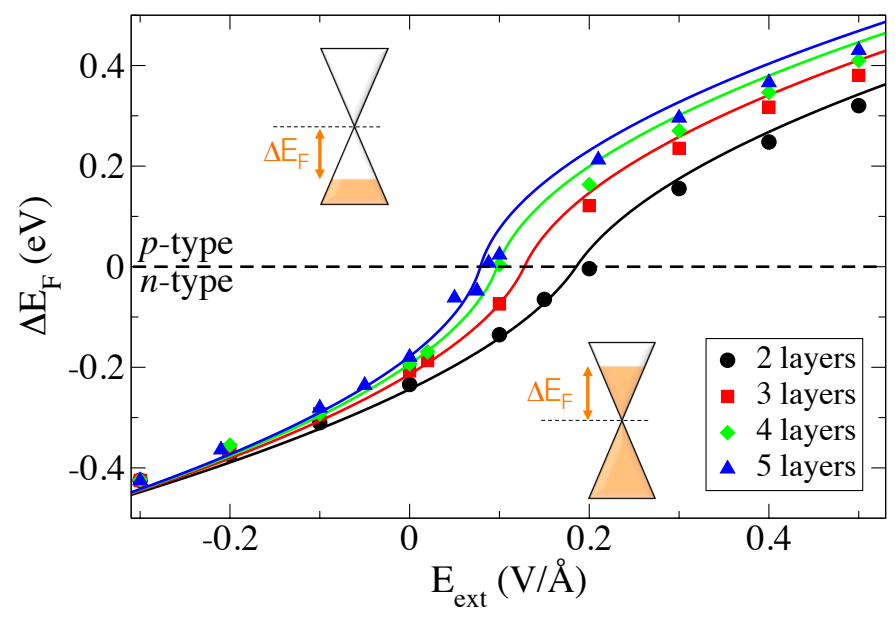

Figure 4.3: Fermi level shift as a function of an external electric field for $\mathrm{Cu}(111) \mid h-$ $\mathrm{BN} \mid$ graphene structures with 2-5 layers of $h$-BN. The symbols indicate calculated DFT values; the lines represent the model of Eq. (4.3).

applying an external electric field that opposes the intrinsic field. With $E_{\text {ext }}=+0.1$ $\mathrm{V} / \AA$ the total internal electric field is zero, and the charge distribution in the $h$-BN slab becomes identical to that of a free-standing $h$-BN slab, see the black line in Fig. 4.2. The green line in Fig. 4.2 shows $\Delta n(z)$ resulting from reversing the external field $E_{\text {ext }}=-0.1 \mathrm{~V} / \AA$, that increases the polarization of the $h$-BN slab.

An external field can also be used to control the position of the Fermi level, i.e. the concentration of charge carriers, in graphene. [53,54] Calculated Fermi level shifts for a range of $h$-BN slab thicknesses and external electric field values are plotted in Fig. 4.3. The curves for different $h$-BN slab thicknesses have a similar, highly nonlinear, shape. Similar shapes of the position of the Fermi level as a function of a gate voltage have been observed in scanning tunneling spectroscopy (STS) experiments, $[69,58,62,63]$ as well as in work function measurements.[70]

The points on the curves where $\Delta E_{\mathrm{F}}=0$ correspond to the charge neutrality level of graphene, i.e. to undoped graphene. At these points the external field $E_{\text {ext }}$ is equivalent to a potential difference $V_{\mathrm{g}}$ across the $h$-BN slab that compensates for $V_{0}$. We expect $\left|E_{\text {ext }}\right| \propto\left|V_{\mathrm{g}}\right| / d$, where $d$ is the thickness of the $h$-BN layer. The external field strength corresponding to the charge neutrality level should then decrease monotonically with increasing slab thickness, as is indeed observed in Fig. 4.3.

\subsection{Model}

To understand the intrinsic doping (Figure 4.1), as well as the external field effect (Figure 4.3) quantitatively, we develop the following analytical model whose parameters are shown in Fig. 4.4. The work function of the metal|dielectric|graphene stack 


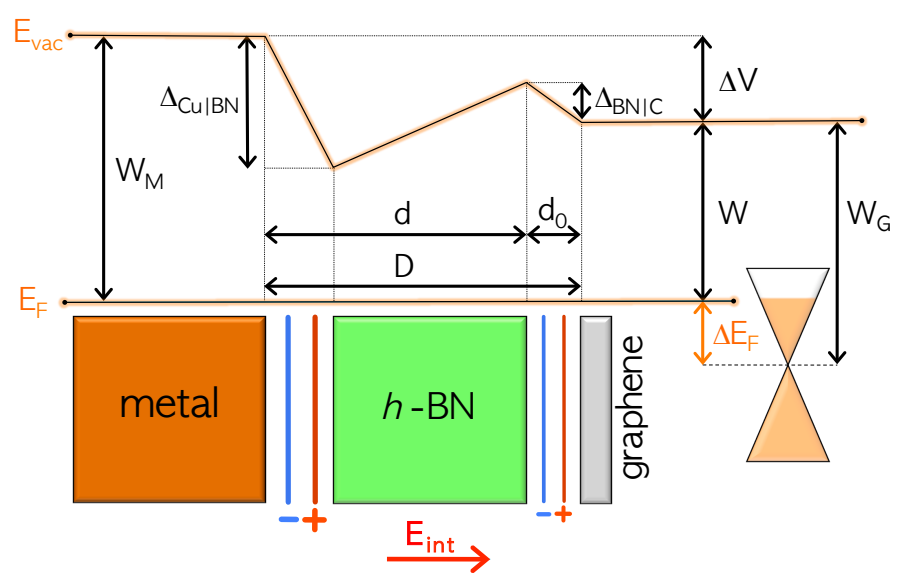

Figure 4.4: Schematic drawing of the metal $\mid h$-BN|graphene structure and the electrostatic potential (energy) across the structure. $E_{\text {int }}$ represents the total electric field across the $h$-BN slab of thickness $d$; the other symbols are explained in the text.

is given by $W=W_{\mathrm{M}}-\Delta V$, with $\Delta V$ the total potential difference across the stack. We model this potential difference as $\Delta V(d)=-e E_{\mathrm{int}} d+\Delta_{\mathrm{Cu} \mid \mathrm{BN}}+\Delta_{\mathrm{BN} \mid \mathrm{C}}$, where $E_{\mathrm{int}}$ is the total electric field inside the dielectric, and $e>0$ is the elementary charge. This field can be related to an externally applied electric field by $\epsilon_{0}\left(E_{\text {ext }}-\kappa E_{\text {int }}\right)=\sigma$, with $\kappa$ the dielectric constant of the dielectric layer and $\sigma$ the surface charge density of the graphene sheet. These relations can be used in Eq. (4.1) to derive a first expression for $\Delta E_{\mathrm{F}}$ in terms of $\sigma$.

A second relation between $\Delta E_{\mathrm{F}}$ and $\sigma$ is obtained by noting that charge in graphene is introduced by (de)populating states away from the charge neutrality point, $\sigma=$ $e \int_{0}^{\Delta E_{\mathrm{F}}} D(E) d E$, and that the density of states near the conical points is well described by a linear function, $D(E)=D_{0}|E| / A$, with $D_{0}=0.09 /\left(\mathrm{eV}^{2}\right.$ unit cell) and $A=5.18 \AA^{2}$ the area of a graphene unit cell. This then gives $\sigma=\operatorname{sign}\left(\Delta E_{\mathrm{F}}\right) \Delta E_{\mathrm{F}}^{2} e D_{0} /(2 A)$. Combining the two relations between $\Delta E_{\mathrm{F}}$ and $\sigma$ gives

$$
\Delta E_{\mathrm{F}}= \pm \frac{\sqrt{1+2 \alpha D_{0} d\left|V_{\mathrm{g}}-V_{0}\right| / \kappa}-1}{\alpha D_{0} d / \kappa}
$$

where $V_{\mathrm{g}}=-e E_{\text {ext }} d / \kappa, V_{0}$ is given by Eq. (4.2), and $\alpha=e^{2} / \epsilon_{0} A=34.93 \mathrm{eV} / \AA$. The sign of $\Delta E_{\mathrm{F}}$ is determined by the sign of $V_{0}-V_{\mathrm{g}}$. Equation (4.3) describes how the Fermi level in graphene depends on the gate voltage $V_{\mathrm{g}}$ (or the external electric field $\left.E_{\text {ext }}\right)$ and the thickness $d$ of the dielectric layer. We define $d$ as the separation $D$ between the top Cu layer and the graphene sheet minus a correction $d_{0}$, because most of the displaced charge is localized between $\mathrm{Cu}$ and $h$-BN and between $h$-BN and graphene, see Fig. 4.2. We have used the value $d_{0}=2.4 \AA$.[73] In principle the dielectric constant of the $h$-BN layer $\kappa$ is a (weak) function of its thickness. We have used the constant value $\kappa=2.72$, calculated for a two-layer $h$-BN slab. 
The results of this model are given in Figs. 4.1 and 4.3. The agreement with the results from the DFT calculations for the $\mathrm{Cu}(111) \mid h$-BN|graphene structures is very good. Note that the model has no adjustable parameters, as quantities such as $V_{0}$ (Eq. (4.2)) are obtained from separate calculations. The model correctly describes the intrinsic doping and its dependence on the $h$-BN layer thickness, Fig. 4.1, as well as the dependence of the doping on the external field, Fig. 4.3.

\subsection{Summary.}

The doping of graphene in $\mathrm{Cu}(111) \mid h$-BN|graphene structures is studied by monitoring the Fermi level shift by means of first-principles DFT calculations. We predict that graphene is intrinsically $n$-type doped for sufficiently thin $h$-BN layers. This is due to the potential difference between $\mathrm{Cu}$ and graphene arising from substantial dipole layers at the $\mathrm{Cu} \mid \mathrm{BN}$ and the $\mathrm{BN} \mid$ graphene interfaces, as well as from the work function difference between $\mathrm{Cu}$ and graphene, Eq. (4.2). The doping level decreases with increasing $h$-BN layer thickness, and approaches zero for thick layers. It can be varied by applying an external electric field and the resulting shift of the Fermi level has a modified square-root like dependence on the field. For thick dielectric spacers, and in the absence of work-function-difference and interface-dipole terms, this is similar to what is observed in experiments.[69, 58, 62, 63, 70] For very thin dielectric layers, these interface terms are predicted to play an important role and both the $h$-BN layer thickness dependence as well as the field dependence of the doping can be described quantitatively by an analytical model, Fig. 4.3. The parameters of this model can be determined experimentally or obtained from DFT calculations on individual surfaces (the work functions of the metal and of graphene), on interfaces (the interface dipoles formed at the $\mathrm{Cu}(111) \mid h$ - $\mathrm{BN}$ and the $h$-BN|graphene interfaces), or on slabs (the dielectric constant of a $h$-BN layer). 


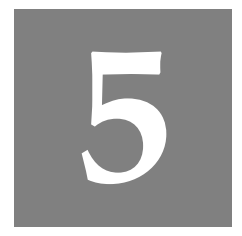

\section{Field effect doping of graphene in metal|dielectric|graphene}

\section{heterostructures: a model based upon first-principles calculations*}

We study how the Fermi energy of a graphene monolayer separated from a conducting substrate by a dielectric spacer depends on the properties of the substrate and on an applied voltage. An analytical model is developed that describes the Fermi level shift as a function of the gate voltage, of the substrate work function, and of the type and thickness of the dielectric spacer. The parameters of this model, that should describe the effect of gate electrodes in field-effect devices, can be obtained from density functional theory (DFT) calculations on single layers or interfaces. The doping of graphene in metal|dielectric|graphene structures is found to be determined not only by the difference in work function between the metal and graphene and the dielectric properties of the spacer but potential steps that result from details of the microscopic bonding at the interfaces also play an important role. The doping levels predicted by the model agree very well with the results obtained from first-principles DFT calculations on metal|dielectric|graphene structures with the metals $\mathrm{Al}, \mathrm{Co}, \mathrm{Ni}, \mathrm{Cu}, \mathrm{Pd}, \mathrm{Ag}$, Pt or $A u$, and a h-BN or vacuum dielectric spacer.

*Based on: M. Bokdam, P.A. Khomyakov, G. Brocks and P.J. Kelly, Field effect doping of graphene in metal|dielectric|graphene heterostructures: a model based upon first-principles calculations, Physical Review B 87, 075414 (2013). 


\subsection{Introduction}

The successful extraction of graphene, single monolayers of graphite, has led to the discovery of new physical phenomena that result from the unique electronic structure of this two-dimensional system.[1] Many of the remarkable properties of graphene have been explored using field effect devices $[53,54]$ in which a graphene flake is placed on top of an oxidized silicon wafer and contacted with metal (source and drain) electrodes.

Direct contact between graphene and a metal electrode generally results in a transfer of electrons between the two materials. Depending upon the metal species used, the contact causes graphene to be doped $n$ - or $p$-type in equilibrium. [72, 73] Metal-contact doping also induces a lateral space charge and corresponding band bending in the graphene adjacent to, but not covered by, the metal contact. The twodimensional character of graphene and the low density of states (Dos) around its Fermi level then result in an extended space charge region.[76] The space charge distribution is inhomogeneous, which can lead to $p-p^{\prime}, n-n^{\prime}, p-n$ or, with gating, to even more complex profiles such as $p-n-p^{\prime}$ within the space charge region.[77, 76, 78]

In this paper we focus on electrostatic doping, where application of a voltage difference between graphene and a back gate changes the position of the Fermi energy with respect to the Dirac point $[53,79,69,58,70,80]$ and a nonlinear shift of the graphene Fermi energy as a function of the gate voltage has been observed in scanning tunneling spectroscopy (STS) experiments[69] and in work function measurements [70]. In experiments such as these, where a $\mathrm{SiO}_{2}$ dielectric was used with a doped $\mathrm{Si}$ gate, it is believed that intrinsic inhomogeneities in $\mathrm{SiO}_{2}$ as well as surface roughness are reasons that much lower charge carrier mobilities are observed than for freely suspended graphene.[81]

In this respect, a much better gate dielectric for graphene based electronics is hexagonal boron-nitride, $h$-BN.[4, 82, 83, 61, 62, 63, 80] Graphene adsorbed on $h$-BN has been shown $[61,62,63]$ to be much flatter than on $\mathrm{SiO}_{2}$ and to have highly mobile charge carriers.[61] This favorable situation can be related to the fact that $h$-BN has a layered crystal structure similar to that of graphite; it consists of atoms that are strongly bonded in plane in a honeycomb lattice, whereas the binding between these planes is much weaker. But where graphene is a semi-metal, $h-\mathrm{BN}$ is an insulator. Graphene binds weakly to $h$-BN with an interaction similar to that between $h$-BN monolayers, or between the graphene layers in graphite.[4] All of this means that adsorbing graphene onto $h$-BN perturbs its unique electronic structure minimally.

Like (multilayer) graphene, layers of $h$-BN can be prepared by micromechanical cleavage,[64] and can be transferred to a metal substrate to form a gate electrode.[84, 85] Alternatively, single layers of $h$-BN have been grown directly on the (111) surface of transition metals such as $\mathrm{Cu}, \mathrm{Pt}, \mathrm{Rh}, \mathrm{Pd}$ and Ni.[86, 87, 88] Graphene $\mid h$-BN heterostructures have been grown on $\mathrm{Ni}(111)$ [89] and more recently on $\mathrm{Ru}(0001)$.[90] Very recently, structures of alternating $h$-BN and graphene layers have been constructed and shown to operate as a field-effect tunneling transistor.[80] 
Obviously the properties of the gate electrode play an important role in determining the behavior of graphene field effect devices. In this paper we develop an analytical model for the electrostatic doping of graphene as a function of the electric field generated by a gate bias and test the model against results obtained using first-principles density functional theory (DFT) calculations on metal gate|dielectric| graphene $(\mathrm{M}|\mathrm{D}| \mathrm{Gr})$ stacks in the presence of an external electric field across the stack. The results of calculations for the particular case of $\mathrm{M}=\mathrm{Cu}$ were recently briefly reported.[83]

The electrostatic model for the $\mathrm{M}|\mathrm{D}| \mathrm{Gr}$ structure is based upon a parallel plate capacitor geometry. The input parameters to the model are the work function of the metal gate, the dielectric constant and thickness of the gate dielectric, and the potential steps formed at the interfaces between the metal and the dielectric, and between the dielectric and the graphene sheet. Such potential steps originate from interactions between the two materials at an interface, and they can make a nonnegligible contribution to the potential profile.[72, 73, 74, 75, 83] All the parameters are accessible to DFT calculations on single layers or interfaces.

In the DFT calculations on the full $\mathrm{M}|\mathrm{D}| \mathrm{Gr}$ structures, we use primarily $h$-BN as dielectric material and study the series of metals $\mathrm{Al}, \mathrm{Co}, \mathrm{Ni}, \mathrm{Cu}, \mathrm{Pd}, \mathrm{Ag}, \mathrm{Pt}$, and $\mathrm{Au}$, whose work functions span a considerable range, from 4.2 to $6.0 \mathrm{eV}$. $h$ - $\mathrm{BN}$ is physisorbed on $\mathrm{Al}, \mathrm{Cu}, \mathrm{Ag}$, $\mathrm{Pt}$, and $\mathrm{Au}$ and chemisorbed on $\mathrm{Co}, \mathrm{Ni}$, and $\mathrm{Pd}$ allowing us to study the effect of the bonding between metal and dielectric on the electrostatic doping of graphene. To assess the influence of the dielectric, we examine the effect of using a vacuum "dielectric" instead of $h$-BN.

The paper is organized as follows. In the next section we formulate the analytical model for electrostatic doping of graphene. We describe the DFT calculations and the (electronic) structures of the metal $\mid h$-BN $\mid$ graphene stacks in Sec. 5.3. The results of the calculations are presented in Sec. 5.4 and compared to the predictions of the model. Finally, Sec. 5.5 presents a summary plus some conclusions.

\subsection{Electrostatic doping model}

We model the $\mathrm{M}|\mathrm{D}| \mathrm{Gr}$ structure as a parallel plate capacitor. Such a model has been used successfully to explain the Fermi level shift in graphene directly absorbed on a metal substrate.[72, 73] Inserting a dielectric is expected to modify the potential profile as shown schematically in Fig. 5.1. The shift of the Fermi level, $\Delta E_{\mathrm{F}}$, with respect to the charge neutrality point in graphene, i.e. the conical points of the band structure, is given by

$$
\Delta E_{\mathrm{F}}=W-W_{\mathrm{Gr}},
$$

with $W_{\mathrm{Gr}}$ and $W$ the work functions of graphene in vacuum, and of the complete $\mathrm{M}|\mathrm{D}| \mathrm{Gr}$ stack, respectively. Note that a negative value of $\Delta E_{\mathrm{F}}$ represents electron doping, a positive value hole doping. $W$ can be written as

$$
W=W_{\mathrm{M}}-\Delta V,
$$




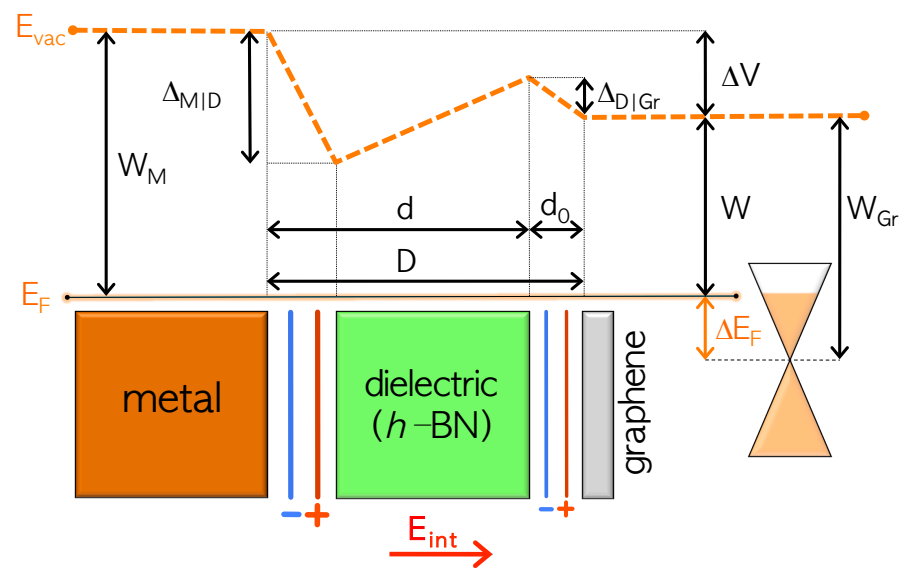

Figure 5.1: Schematic drawing of the metal|dielectric|graphene $(\mathrm{M}|\mathrm{D}| \mathrm{Gr})$ structure. The electrostatic potential profile in the structure is indicated by the wide dashed (orange) line at the top. $\Delta V$ is the shift of the work function of the structure with respect to that of the clean metal, $W_{\mathrm{M}} ; \Delta_{\mathrm{M} \mid \mathrm{D}}, \Delta_{\mathrm{D} \mid \mathrm{Gr}}$ are the potentials steps at the interfaces; $\Delta E_{\mathrm{F}}$ is the Fermi level shift in graphene caused by the electrostatic doping.

with $W_{\mathrm{M}}$ the work function of the clean metal substrate, and $\Delta V$ the potential difference across the stack. The latter will in general be a function of the dielectric thickness $d$.

We model the potential difference as

$$
\Delta V=-e E_{\mathrm{int}} d+\Delta_{\mathrm{M} \mid \mathrm{D}}+\Delta_{\mathrm{D} \mid \mathrm{Gr}},
$$

where $E_{\text {int }}$ is the electric field inside the dielectric, $-e$ is the charge of an electron, and $\Delta_{\mathrm{M} \mid \mathrm{D}}$ and $\Delta_{\mathrm{D} \mid \mathrm{Gr}}$ are potential steps at the metal|dielectric and the dielectric|graphene interfaces, respectively. Interface bonding between two materials usually results in the formation of a dipole layer that is accompanied by a potential step[74, 75, 2] localized at the interface. Interface dipoles are intrinsic properties of interfaces and even if the interaction at an interface is as weak as in the case of physisorption, they can be sizable. We will assume that interface potential steps are independent of applied electric fields for field magnitudes of practical interest and test this assumption with DFT calculations.

In the absence of a dielectric spacer, an externally applied gate voltage gives rise to an electric field $E_{\text {ext }}$. In the following, it will be convenient to use $E_{\text {ext }}$ as an independent variable. The internal field, Eq. (5.3), is obtained from the standard electrostatic boundary condition

$$
\epsilon_{0}\left(E_{\text {ext }}-\kappa E_{\mathrm{int}}\right)=\sigma,
$$

with $\kappa$ the relative dielectric constant of the dielectric layer, and $\sigma$ the surface charge 
density of the graphene sheet.

The latter can be related to the Fermi level shift $\Delta E_{\mathrm{F}}$ by noting that charge in graphene is introduced by occupying states starting from the charge neutrality point, $\sigma=e \int_{0}^{\Delta E_{\mathrm{F}}} D(E) d E$, where the charge neutrality point corresponds to the conical points in the graphene band structure. The density of states near the conical points is described well by the linear function, $D(E)=|E| D_{0} / A$, with $D_{0}=0.102 /\left(\mathrm{eV}^{2}\right.$ unit cell), and $A=5.18 \AA^{2}$ as the area of a graphene unit cell, so that

$$
\sigma=\operatorname{sign}\left(\Delta E_{\mathrm{F}}\right) \frac{e D_{0}}{2 A}\left(\Delta E_{\mathrm{F}}\right)^{2}
$$

The value of $D_{0}^{\dagger}$ is sensitive to the k-point grid used for the Brillouin zone sampling, and it depends on the substrate used; technical details are discussed in appendix A.

Equations (5.1)-(5.5) can be combined to give a closed expression for $\Delta E_{\mathrm{F}}$ in terms of the applied external field $E_{\text {ext }}$

$$
\Delta E_{\mathrm{F}}\left(E_{\text {ext }}\right)= \pm \frac{\sqrt{1+2 D_{0} \alpha(d / \kappa)^{2}\left|e\left(E_{\text {ext }}+E_{0}\right)\right|}-1}{D_{0} \alpha d / \kappa},
$$

where $\alpha=e^{2} /\left(\epsilon_{0} A\right)=34.93 \mathrm{eV} / \AA$ for a $1 \times 1$ graphene unit cell. The sign of $\Delta E_{\mathrm{F}}$ is determined by the sign of $E_{\text {ext }}+E_{0}$, where

$$
E_{0}=V_{0} \frac{\kappa}{d e}
$$

with

$$
V_{0}=W_{\mathrm{M}}-W_{\mathrm{Gr}}-\Delta_{\mathrm{M} \mid \mathrm{D}}-\Delta_{\mathrm{D} \mid \mathrm{Gr}} .
$$

$E_{0}$ is the value of the intrinsic electric field across the dielectric in the absence of any external electric field, see Fig. 5.1, and it can be substantial when $d$ is sufficiently small, i.e., for thin dielectric spacers. It results from an electron transfer in equilibrium between the metal and graphene across the dielectric spacer that leads to an intrinsic doping of graphene. A compensating external field $E_{\text {ext }}=-E_{0}$ is required to bring the Fermi level to the conical points and make graphene electrically neutral, $\Delta E_{\mathrm{F}}=0$.

According to Eqs. (5.1)-(5.3), the Fermi level shift $\Delta E_{\mathrm{F}}$ and the internal electric field $E_{\text {int }}$ are linked by a linear relation. This means that both these quantities are similar non-linear functions of the external electric field $E_{\text {ext }}$, Eq. (5.6), whose nonlinearity is determined by the doping charge on graphene. Figure 5.2 shows $E_{\text {int }}$ as a function of $E_{\text {ext }}$ for two different values of the dielectric constant $\kappa$. The points in these curves where the derivative is maximal correspond to undoped graphene, i.e., $\Delta E_{\mathrm{F}}=0$ and $E_{\text {ext }}=-E_{0}$. It follows from Eq. (5.4) that at these points the curves

†The value that was used for $D_{0}$ in Chapter 4 and Ref. [83], $D_{0}=0.9 /\left(\mathrm{eV}^{2}\right.$ unit cell), corresponds to the value calculated for freestanding graphene with a $36 \times 36$ k-point grid, see Table A.1. We use a slightly different value in this paper to be internally consistent with the explicit DFT calculations for $h$-BN performed with a $36 \times 36 \mathrm{k}$-point grid. 


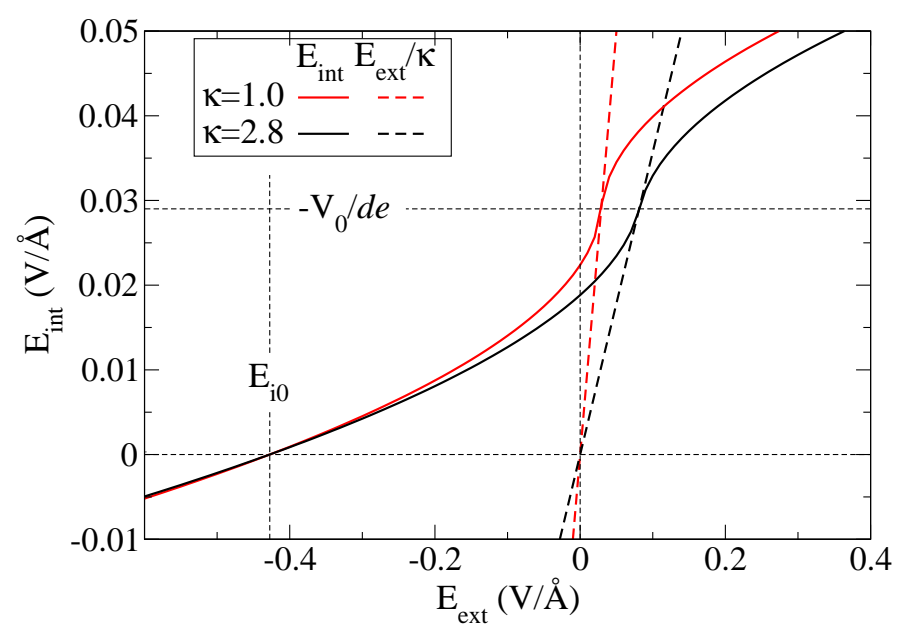

Figure 5.2: The internal field $E_{\text {int }}$ (solid lines) inside the dielectric spacer of Fig. 5.1, as a function of the applied external field $E_{\text {ext }}$, for two values of $\kappa$. The black line corresponds to a $\mathrm{Cu}|h-\mathrm{BN}|$ Graphene structure with 5 layers of $h-\mathrm{BN}$ and $\kappa=2.8$. The red line would be the response of the same system, but with $\kappa=1.0$ instead of 2.8. The curves cross at $E_{\text {ext }}=E_{\mathrm{i} 0}$, see Eq. (5.9), where $E_{\mathrm{int}}=0$. At fields $E_{\mathrm{ext}}=-E_{0}$, see Eq. (5.7), the curves cross the lines $E_{\text {ext }} / \kappa$ (dashed lines).

cross the lines $E_{\text {ext }} / \kappa$, see Fig. 5.2. From Eq. (5.7) it follows that the corresponding internal electric field is $E_{\text {int }}=-V_{0} / d e$.

The $E_{\text {int }}$ curves cross at $E_{\text {int }}=0$. From Eqs. (5.1)-(5.5) it is easily seen that this occurs when $E_{\text {ext }}=E_{\mathrm{i} 0}$, where

$$
E_{\mathrm{i} 0}=V_{0}\left|V_{0}\right| \frac{e D_{0}}{2 A \epsilon_{0}} .
$$

The corresponding Fermi level shift

$$
\Delta E_{\mathrm{F}}\left(E_{\mathrm{i} 0}\right)=V_{0},
$$

is independent of $d$ and $\kappa$, i.e., all curves $\Delta E_{\mathrm{F}}\left(E_{\text {ext }}\right)$ for different dielectric thicknesses cross at $\Delta E_{\mathrm{F}}=V_{0}$ for $E_{\text {ext }}=E_{\mathrm{i} 0}$. This crossing point could be used to determine $V_{0}$ from experiment.

Equation (5.6) constitutes the model we will use in the remainder of this paper. Assuming that the properties of graphene are known, the model depends on the parameters $W_{\mathrm{M}}, \Delta_{\mathrm{M} \mid \mathrm{D}}, \Delta_{\mathrm{D} \mid \mathrm{Gr}}$, and $\kappa$. All of these parameters can be obtained from DFT calculations. The thickness of the dielectric spacer $d$ is somewhat ill-defined at the atomic level. As in Ref. [73], we set $d=D-d_{0}$, where $D$ is the distance from the center of the top layer of metal atoms to the graphene plane, and $d_{0}=2.4 \AA$.

The model can also be expressed in terms of an applied gate voltage, writing the 
latter as

$$
V_{\mathrm{g}}=-e E_{\mathrm{ext}} d / \kappa
$$

Equation (5.6) then becomes

$$
\Delta E_{\mathrm{F}}= \pm \frac{\sqrt{1+2 \alpha D_{0} d\left|V_{\mathrm{g}}-V_{0}\right| / \kappa}-1}{\alpha D_{0} d / \kappa},
$$

and the sign of $\Delta E_{\mathrm{F}}$ is determined by the sign of $V_{0}-V_{\mathrm{g}}$.

\subsection{Computational details}

In this section we briefly describe the most salient features of the first-principles DFT calculations that we use to determine the parameters entering the model defined in the previous section. We calculate electronic ground state energies within the framework of the plane-wave PAW pseudopotential method,[14] at the level of the local density approximation (LDA),[16] as implemented in VASP $[12,44,45]$ and use them to optimize the geometries of $\mathrm{M}|\mathrm{D}| \mathrm{Gr}$ structures for a number of representative metals. The structures are modeled as periodic slabs separated by a vacuum region $\sim 15 \AA$ thick. A dipole correction is applied to avoid spurious interactions between periodic images of the slab.[91] A plane wave kinetic energy cutoff is set at $400 \mathrm{eV}$.

A $36 \times 36$ k-point grid is used to sample the surface Brillouin Zone (BZ) and structural relaxations are performed using a smearing method for the BZ integration,[92] with a smearing parameter of $0.2 \mathrm{eV}$. Total energies and charge densities of optimized structures are then recalculated using the tetrahedron scheme. The special points $\Gamma, K, K^{\prime}$ and $M$ of the graphene band structure are explicitly included in the BZ sampling, which is important for an accurate description of the electronic structure around these points.

The electronic self-consistency criterion is set to $10^{-8} \mathrm{eV}$. Such a strict criterion is required to assure convergence of the charge distribution. We consider only closepacked metal surfaces with three-fold rotational symmetry, i.e., the (111) surface for fcc metals, and the (0001) surface for hcp metals. A slab of six layers of atoms is used to represent the metal surface. The $h$-BN layers are adsorbed on one side of the slab to represent the dielectric, and graphene is adsorbed on top of this. To model the effect of the gate potential, a sawtooth-shaped potential is applied across the whole slab.[93] The carbon atoms in graphene, the boron and nitrogen atoms in the $h$-BN slab, and the separation between the top two atomic layers of the metal were allowed to relax during the geometry optimization until the total energies were converged to within $10^{-7} \mathrm{eV}$.

The LDA is found to give a reasonable description of the weak interaction between $h$-BN layers and between a $h$-BN layer and graphene.[4] Likewise, LDA describes the interaction between graphene and metal surfaces reasonably well.[72, 73, 76] We expect a comparably good description of the interaction between $h$-BN and metal surfaces and use the LDA functional throughout. 
For metal $\mid h$-BN interface calculations, we fixed the lattice constant of $h$-BN to its optimized LDA value of $a_{\mathrm{hex}}=2.49 \AA$. The in-plane lattice parameter of the metal surfaces was then adjusted so that for $\mathrm{Cu}, \mathrm{Ni}$ and $\mathrm{Co}$ we could use a common $1 \times 1$ surface unit cell, Fig. 5.3a). For $\mathrm{Au}, \mathrm{Pt}, \mathrm{Al}, \mathrm{Ag}$, and $\mathrm{Pd}$, a $\sqrt{3} \times \sqrt{3}$ surface unit cell matches very well to a $2 \times 2$ surface unit cell of $h$-BN, Fig. $5.3 b$ ). Adopting this to model the interface involves changing the in-plane lattice parameter of the metals by less than $2 \%$ compared to the optimized values. Such a change does not greatly perturb the electronic structure of the metals, especially after allowing the top two metal layers to relax in a direction perpendicular to the surface. The small change in the metal lattice parameter is found to have only a small effect on the properties we are interested in. For instance, the work function of the $\mathrm{Cu}(111)$ surface is increased by a mere $0.05 \mathrm{eV}$ and the density of states at the Fermi level is unaltered.

In the most stable configuration of a $h-\mathrm{BN}$ sheet on $\mathrm{Cu}, \mathrm{Ni}$ and $\mathrm{Co}$, nitrogen atoms are adsorbed on top of metal surface atoms and boron atoms are at hollow sites as shown schematically in Fig. 5.3a). The most stable structure for $h$-BN on Au, Pt, $\mathrm{Al}$, $\mathrm{Ag}$, and Pd is shown in Fig. 5.3b). In an interface unit cell containing 3 surface metal atoms, 4 boron and 4 nitrogen atoms, one boron and one nitrogen atom is absorbed on top of a metal surface atom; all other boron and nitrogen atoms are on bridge sites. These structures are used to calculate the metal $\mid h-\mathrm{BN}$ interface dipoles.

For calculations on metal $|h-\mathrm{BN}|$ graphene stacks we use the structures shown schematically in Fig. 5.3. Here we choose the lattice constant of graphene equal to its

a)

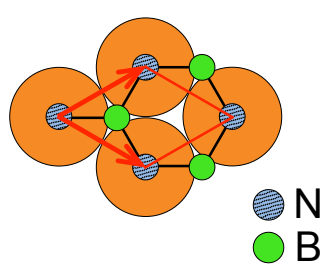

b)

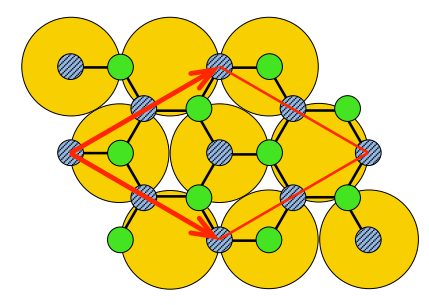

c)

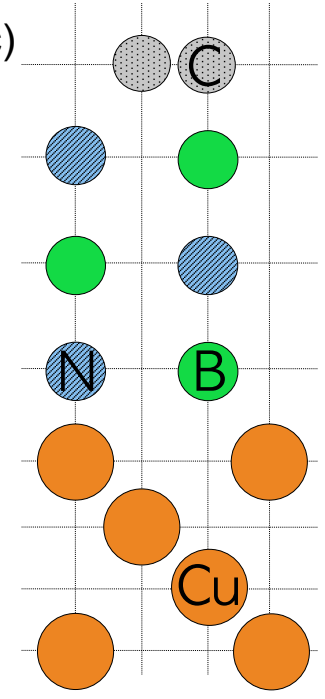

d)

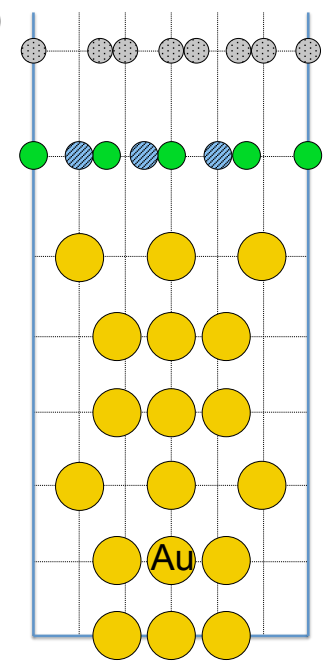

Figure 5.3: Top views of the energetically favorable configurations of $h$-BN on a) $\mathrm{Cu}$, $\mathrm{Ni}(111), \mathrm{Co}(0001)$, and b) $\mathrm{Pt}, \mathrm{Pd}, \mathrm{Au}, \mathrm{Ag}, \mathrm{Al}(111)$ in $1 \times 1$ and $\sqrt{3} \times \sqrt{3}$ surface unit cells, respectively. And the corresponding side views, c) $\mathrm{Cu}, \mathrm{Ni}, \mathrm{Co}$ and d) $\mathrm{Au}, \mathrm{Pt}$, Ag, Pd, Al. 
optimized LDA value $a=2.445 \AA$, and adapt the in-plane lattice constants of $h$-BN and the metal accordingly. This means that the $h-\mathrm{BN}$ is compressed in plane by $1.8 \%$ and the metal by up to $3.8 \%$. The same procedure was used in our earlier studies of graphene on metal surfaces[73] and on bulk $h$-BN, [4] the basic rationale being that a change of the in-plane lattice parameter of the metal or of $h$-BN will have a smaller effect on their electronic structures (at the Fermi energy) than a corresponding change in graphene would have on its properties. For instance, compressing the $\mathrm{Cu}(111)$ lattice by $4 \%$ increases its work function by only $0.08 \mathrm{eV}$. As a second example, the potential step at the $\mathrm{Cu}(111) \mid h$ - $\mathrm{BN}$ interface is $\Delta_{\mathrm{Cu} \mid \mathrm{BN}}=1.12 \mathrm{eV}$ for $a_{\text {hex }}=2.445 \AA$, the lattice constant of graphene, and $1.18 \mathrm{eV}$ for $a_{\text {hex }}=2.49 \AA$, the $h$-BN lattice constant.

$h$-BN layers are stacked in $\mathrm{AA}^{\prime}$ fashion, with boron on top of nitrogen and vice versa, see Fig. 5.3c). The graphene layer is placed on top of $h$-BN as in Ref. [4]. The optimized LDA interlayer separation in the $h$-BN slab is $3.24 \AA$, which is similar to what is found in bulk $h$-BN. For the $\mathrm{Au}(111) \mid$ monolayer $h$-BN|graphene structure (Fig. 5.3d) the calculated equilibrium separation between the top $h$-BN layer and graphene is $3.21 \AA$, which is similar to the calculated separation of $3.22 \AA$ between graphene and bulk $h$-BN, [4] as expected.

In the analytical model outlined in Sec. 5.2 we have assumed that, apart from a shift in the Fermi level, the electronic structure of graphene in a metal $\mid h$-BN|graphene stack is unchanged compared to a free-standing sheet of graphene. We can check this statement explicitly by analyzing the electronic structure obtained from a DFT calculation on a $\mathrm{M}|h-\mathrm{BN}| \mathrm{Gr}$ stack. As an example, the left panel of Fig. 5.4 shows the band structure of a $\mathrm{Au}(111)$ substrate with a monolayer of $h$-BN and graphene on top. The graphene and $h$ - $\mathrm{BN} p_{z}$ character of the eigenstates is indicated to help identify the bands. For comparison, the electronic structures of free-standing graphene and of a $h$-BN monolayer are shown superimposed in the right panel of Fig. 5.4. To make the Dirac points of graphene coincide in energy, the band structure of free standing graphene in the right panel has been shifted downwards by $0.08 \mathrm{eV}$ corresponding to the doping level of graphene in the $\mathrm{Au} \mid h-\mathrm{BN}$ monolayer|graphene structure. The top of the $h$-BN valence band, at $\mathrm{K}$, is positioned at $-1.28 \mathrm{eV}$, corresponding to the Schottky barrier for holes found for the $\mathrm{Au} \mid h-\mathrm{BN}$ structure. Apart from these energy shifts, the band structures of both graphene and $h$-BN in the $\mathrm{Au} \mid h$-BN|graphene stack are hardly changed with respect to their free-standing counterparts.

This reflects the weakness of the interaction between $\mathrm{Au}$ and $h-\mathrm{BN}$, and between $h$-BN and graphene. Some metal substrates have a stronger interaction with $h$-BN which does affect the $h$-BN derived bands. However, as far as the doping levels of graphene are concerned, the effects of that interaction can be fully incorporated in the potential step $\Delta_{\mathrm{M} \mid \mathrm{BN}}$ associated with the metal $\mid h$-BN interface that will be discussed in the next section. If the $h$-BN and the graphene lattices are assumed to be commensurate, the sublattice-symmetry-breaking potential of $h$-BN induces a small band gap of $\sim 54 \mathrm{meV}$ between the tips of the Dirac cones;[4] apart from the formation of this small gap, the DoS remains linear in energy. If the lattices are 


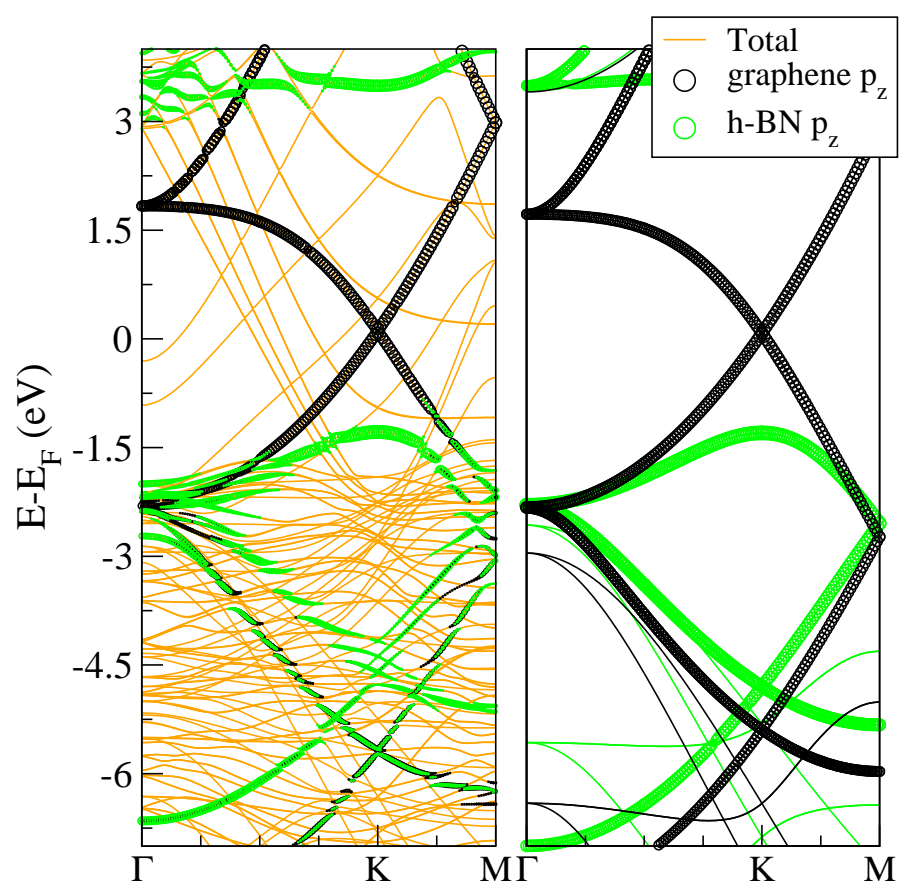

Figure 5.4: Left panel: the band structure of a $\mathrm{Au}(111) \mid h$-BN monolayer|graphene stack. The eigenstates of graphene and $h$-BN with strong $\pi$ character are highlighted in black and green (gray), respectively where the symbol sizes represent the amount of $p_{z}$ character. The plotting order is total, graphene $p_{z}, h-\mathrm{BN} p_{z}$. Right panel: the superimposed band structures of free-standing graphene and a $h$-BN monolayer. The graphene $(h-\mathrm{BN})$ bands have been shifted up (down) by $0.08(1.28) \mathrm{eV}$.

incommensurate, this gap disappears.[62, 61, 63, 94] In the following this point will not be important.

\subsection{Results}

Conceptually, the simplest model we can consider for a study of electrostatic doping of graphene based upon first-principles calculations is a metal|vacuum|graphene stack. Fig. 5.5 shows the shift of the Fermi level in graphene calculated using Eq. (5.1), as a function of the external electric field for the $\mathrm{Cu}(111)$ |vacuum|graphene heterostructure, where the vacuum "thickness" $d=7.18 \AA$. The points in this figure are the calculated DFT values, the solid line represents the model described by Eqs. (5.6) and (5.7) with $\kappa=1$ and $\Delta_{\mathrm{M} \mid \mathrm{D}}=\Delta_{\mathrm{D} \mid \mathrm{Gr}}=0$ for the vacuum dielectric. The match between the model (solid curve) and the DFT values (squares) is excellent.

At zero external bias, the work function difference between the $\mathrm{Cu}(111)$ surface and graphene sheet transfers electrons from one to the other until equilibrium 


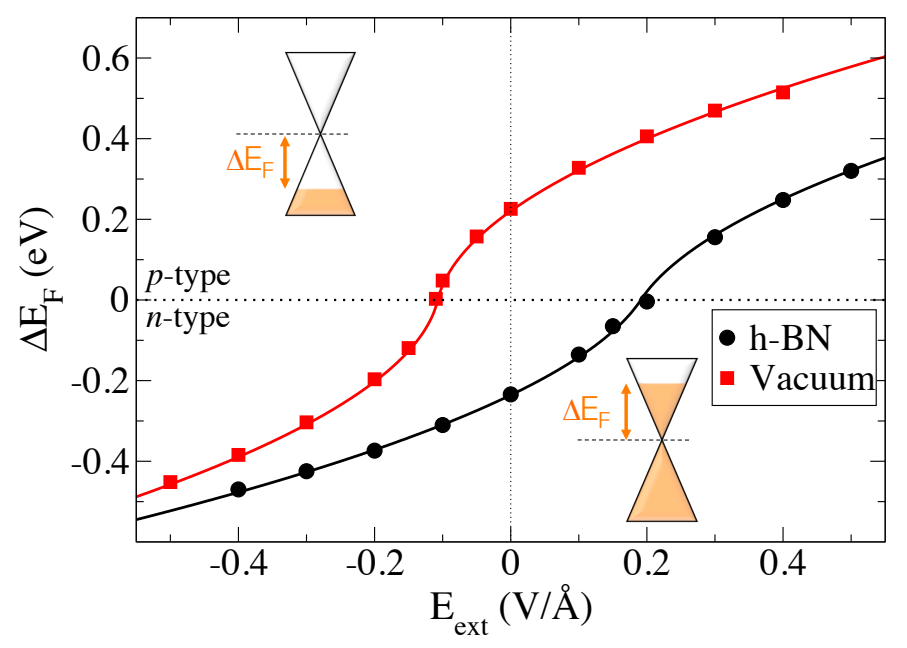

Figure 5.5: The Fermi level shift versus external electric field strength for a $\mathrm{Cu}(111) \mid h$ $\mathrm{BN} \mid$ graphene structure with two layers of $h$-BN (black) and for the same structure with the $h$-BN layers replaced by vacuum (red). The lines represent the model described by Eq.(5.6) with $\kappa=2.80$ and 1.0, respectively. The symbols are the values calculated with DFT.

is established. The transferred charge sets up an intrinsic electric field $E_{0}$ that is determined by the work function difference, see Eq. (5.7). The work functions of the clean $\mathrm{Cu}(111)$ surface and free-standing graphene calculated in the LDA are $5.25 \mathrm{eV}$ and $4.48 \mathrm{eV}$, respectively, which indicates that at zero external bias electrons are transferred from graphene to $\mathrm{Cu}$ so that graphene is doped $p$-type. A compensating negative external field can be applied to undo the graphene doping so $\Delta E_{\mathrm{F}}=0$. The values given by the model of the compensating field and the Fermi level shift at zero bias are in excellent agreement with the DFT results on the $\mathrm{Cu}(111) \mid$ vacuum $\mid$ graphene system, see Fig. 5.5.

Insertion of a $h$-BN dielectric spacer between the $\mathrm{Cu}$ and graphene yields a system that can be realized in practice. The Fermi level shift in graphene for a $\mathrm{Cu}(111) \mid h-$ $\mathrm{BN} \mid$ graphene structure with two layers of $h$-BN (corresponding, by construction, precisely to the vacuum thickness used in the previous calculation) is also shown in Fig. 5.5 as a function of the external electric field. Comparing the results obtained with a $h$-BN spacer and a vacuum spacer, two qualitative differences are immediately obvious. The curve calculated with $h$-BN results is shifted to the right, and it is "flatter".

The latter effect results largely from the dielectric constant of the spacer; Eq. (5.6) shows that $\Delta E_{\mathrm{F}}$ decreases with increasing dielectric constant. We determined the dielectric constant of $h$-BN from calculations on very thin $h$-BN films as the ratio between an applied external electric field and the internal electric field inside the slab [95] determined from the shift of the 1s Nitrogen core levels. Because these levels 
are localized on each layer, their energies shift according to the local electrostatic potential. The shift is linear in the position of the layer, and the slope yields the internal electric field. From calculations on $h$-BN slabs consisting of 2-10 layers in electric fields in the range $0.05-0.45 \mathrm{~V} / \AA$, we find $\kappa=2.80 \pm 0.02$. In our modelling in the remainder of this paper, we use $\kappa=2.80$. Fig. 5.5 shows that with this value of $\kappa$ the shape of the curve gives an excellent description of the DFT results for the $\mathrm{Cu}(111)|h-\mathrm{BN}|$ graphene heterostructure.

The shift between the two curves in Fig. 5.5 results from potential steps $\Delta_{\mathrm{M} \mid \mathrm{D}}$ and $\Delta_{\mathrm{D} \mid \mathrm{Gr}}$ that are formed at the metal $\mid h-\mathrm{BN}$ and $h$-BN|graphene interfaces, respectively. These potential steps are sufficiently large not only to compensate for the difference in work functions of the $\mathrm{Cu}(111)$ surface and free-standing graphene, but even to reverse the intrinsic electric field (Eq. (5.7)). It means that in the $\mathrm{Cu}(111) \mid h$ $\mathrm{BN}$ |graphene stack at zero bias, graphene is $n$-type doped, and one needs to apply a positive external field to undo the graphene doping and enforce $\Delta E_{\mathrm{F}}=0$. Because the interface potential steps play such an important role, we study them in more detail in the next subsection.

\subsubsection{Potential steps at metal $\mid h-\mathrm{BN}$ and $h$-BN $\mid$ graphene interfaces}

In general, potential steps are formed at interfaces between different materials. They result from interface dipole layers, caused by chemical interactions and/or charge transfer between two materials comprising an interface. Substantial interface dipoles can be formed even if the interaction between the two materials is relatively weak as happens when noble gas atoms are adsorbed on, or closed-shell molecules are physisorbed on metal surfaces.[96, 74, 75, 83] Even in the absence of a direct interaction, the difference between the work functions of two metals leads to charge transfer and the formation of contact potentials.

The potential step $\Delta_{\mathrm{M} \mid \mathrm{D}}$ at the metal|dielectric interface can be obtained by comparing the work function $W_{\mathrm{M}}$ of the clean metal surface without the adsorbate to that of the metal with the dielectric adsorbed on it, $W_{\mathrm{M} \mid \mathrm{D}}$. Similarly, the potential step $\Delta_{\mathrm{D} \mid \mathrm{Gr}}$ is given by the difference between the work functions on the dielectric side and the graphene side of a $\mathrm{D} \mid \mathrm{Gr}$ slab

$$
\Delta_{\mathrm{M} \mid \mathrm{D}}=W_{\mathrm{M}}-W_{\mathrm{M} \mid \mathrm{D}} ; \quad \Delta_{\mathrm{D} \mid \mathrm{Gr}}=W_{\mathrm{D} \mid \mathrm{Gr}}-W_{\mathrm{Gr} \mid \mathrm{D}} .
$$

It turns out to be sufficient to study graphene adsorbed on two layers of $h$-BN to find a converged value for $\Delta_{\mathrm{BN} \mid \mathrm{Gr}}=0.14 \mathrm{eV}$. Similarly, a single $h$-BN layer adsorbed on a metal substrate is sufficient to determine $\Delta_{\mathrm{M} \mid \mathrm{BN}}$, demonstrating the true interface nature of these potential steps. The values of $\Delta_{\mathrm{M} \mid \mathrm{BN}}$ calculated for the metals considered in this paper, given in Table 5.1, are seen to be quite substantial, ranging from $0.4 \mathrm{eV}$ for $\mathrm{Al}(111) \mid h$-BN to $1.8 \mathrm{eV}$ for $\mathrm{Co}(0001) \mid h$-BN. In all cases $\Delta_{\mathrm{M} \mid \mathrm{BN}}$ is positive, meaning that adsorption of $h$-BN on a metal substrate lowers the work function. In other words, the interaction between $h$-BN and the metal substrate leads to a net displacement of the electronic charge distribution into the metal. 
Table 5.1: Calculated and experimental potential steps $\Delta_{\mathrm{M} \mid \mathrm{BN}}$ at the metal $\mid h$-BN interface. $d_{e q}$ is the equilibrium separation of the $h$-BN layer from the metal surface. The $h$-BN lattice constant $a_{\text {hex }}=2.49 \AA$ is used for the surface unit cells, except in the last two rows, where the graphene lattice constant $a_{\text {hex }}=2.445 \AA$ is used.

\begin{tabular}{lcccc}
\hline $\mathrm{M}$ & $\Delta_{\mathrm{M} \mid \mathrm{BN}}(\mathrm{eV})$ & $\Delta_{\mathrm{M} \mid \mathrm{BN}}^{\mathrm{exp}}(\mathrm{eV})$ & $d_{\mathrm{eq}}(\AA)$ & $W_{\mathrm{M}}(\mathrm{eV})$ \\
\hline $\mathrm{Co}$ & 1.80 & & 1.92 & 5.52 \\
$\mathrm{Ni}$ & 1.73 & $1.5-1.8^{a, c}$ & 1.96 & 5.52 \\
$\mathrm{Pd}$ & 1.25 & $1.3^{b}$ & 2.47 & 5.53 \\
$\mathrm{Cu}$ & 1.18 & $0.24^{c}, 0.8-1.1^{d}$ & 2.97 & 5.17 \\
$\mathrm{Pt}$ & 1.04 & $0.9^{b}$ & 3.04 & 5.98 \\
$\mathrm{Ag}$ & 0.83 & & 3.20 & 4.83 \\
$\mathrm{Au}$ & 0.79 & & 3.24 & 5.55 \\
$\mathrm{Al}$ & 0.41 & & 3.55 & 4.25 \\
$\mathrm{Gr}$ & 0.11 & & 3.21 & 4.63 \\
\hline $\mathrm{Gr}^{\prime}$ & 0.14 & & 3.22 & 4.48 \\
$\mathrm{Cu}^{\prime}$ & 1.12 & $0.24^{c}, 0.8-1.1^{d}$ & 3.11 & 5.25 \\
\hline
\end{tabular}

${ }^{a}$ Refs. [98, 99, 100], ${ }^{b}$ Ref. [98], ${ }^{c}$ Ref. [87], ${ }^{d}$ Ref. [97].

The metal $\mid h$-BN equilibrium distance $d_{\text {eq }}$ indicates that there are two different adsorption regimes. Adsorption of $h$-BN on the $\mathrm{Co}(0001), \mathrm{Ni}(111)$ and $\mathrm{Pd}(111)$ surfaces leads to equilibrium distances $d_{\mathrm{eq}}<2.5 \AA$, values which are typically associated with chemisorption, whereas the values of $d_{\mathrm{eq}}>3.0 \AA$ for adsorption on the $\mathrm{Cu}, \mathrm{Pt}$, $\mathrm{Ag}, \mathrm{Au}$, and $\mathrm{Al}(111)$ surfaces are typical of physisorption.

The calculated values of $\Delta_{\mathrm{M} \mid \mathrm{BN}}$ increase with decreasing separation of the $h$-BN plane from the metal substrate, as shown in Table 5.1. A smaller separation leads to an increased metal $\mid h$-BN interaction, and hence to a larger deformation of the charge density and a larger potential step at the interface. The calculated $\Delta_{\mathrm{M} \mid \mathrm{BN}}$ generally agree well with the experimental values. However, for $\mathrm{Cu} \mid \mathrm{BN}$, different experiments report quite different results.[87, 97] The values we calculate are in better agreement with values recently reported in Ref. [97]. In view of the results shown in Table 5.1, the experimental value $\Delta_{\mathrm{Cu} \mid \mathrm{BN}}=0.24 \mathrm{eV}$ reported in Ref. [87] is strikingly low.

An interface potential step can have different origins. Chemical interactions between two materials can result in an ordered interface layer of bond dipoles.[2, 101] Even when the interaction is weak, as in the case of physisorption, it is argued that the Pauli exchange interaction between the adsorbate and the substrate can distort the charge density distribution significantly and drive a net displacement of charge towards the "softer" material, in this case the metal substrate, which lowers the work function. This is commonly called the "pushback" or "pillow" effect.[96]

In addition to a potential step induced by a direct interaction at the interface, one can have a contribution originating from an electron transfer to equilibrate the 


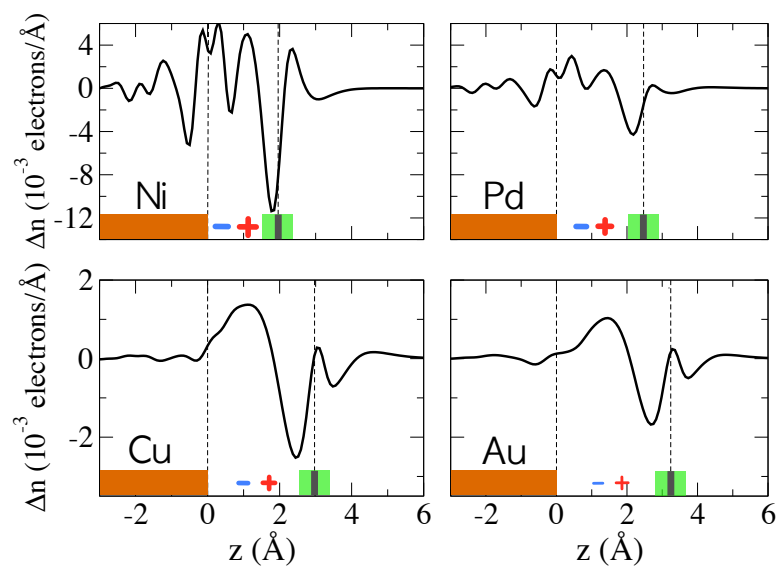

Figure 5.6: Plane-averaged electron density difference $\Delta n_{\mathrm{M} \mid \mathrm{BN}}(z, 0)$ for $\mathrm{Ni}, \mathrm{Pd}, \mathrm{Cu}$ and $\mathrm{Au}$ (111) surfaces covered with a monolayer of $h$-BN. The vertical dashed lines represent the positions of the atomic layers at the interfaces. The position $z$ is measured from the top metal atom.

Fermi level between the two materials on either side of the interface. Such a transfer obviously occurs only if the Fermi level is in a range where the density of states of both materials is non-zero. At interfaces between a metal and an organic molecular material, where charge is exchanged between strong donor or acceptor molecules and the metal surface, this leads to pinning of the Fermi level by the molecules.[102]

One does not expect this to be the case for $h$-BN adsorbed on a metal surface. $h$-BN is a large band gap insulator, and in most cases the Fermi level of the metal is well within the $h$-BN band gap. The potential steps $\Delta_{\mathrm{M} \mid \mathrm{BN}}$ and $\Delta_{\mathrm{BN} \mid \mathrm{Gr}}$ can therefore be ascribed to the direct interaction between the materials at the interface.

We can visualize the electronic displacement and dipole formation at the interface by looking at the electron density of the entire system minus the electron densities of the two separate constituent materials. As only the component perpendicular to the interface is relevant in the present context, it is convenient to work with planeaveraged electron densities $n(z)=\int n(x, y, z) d x d y / A$ in terms of which the electron charge density $\rho=-e n$. The electron displacement in a metal $\mid h-\mathrm{BN}$ structure is defined as

$$
\Delta n_{\mathrm{M} \mid \mathrm{BN}}\left(z, E_{\mathrm{ext}}\right)=n_{\mathrm{M} \mid \mathrm{BN}}\left(z, E_{\text {ext }}\right)-n_{\mathrm{M}}\left(z, E_{\text {ext }}\right)-n_{\mathrm{BN}}\left(z, E_{\text {ext }}\right),
$$

where $n_{\mathrm{M} \mid \mathrm{BN}}$ is the electron density of the composite system, $n_{\mathrm{M}}$ and $n_{\mathrm{BN}}$ are the electron densities of the clean metal substrate and the isolated $h$-BN layer, respectively, and we introduce an explicit $E_{\text {ext }}$ dependence because we wish to study the dependence of the interface electronic displacement on the external electric field. Any electronic displacement $\Delta n(z)$ can be related to a potential (energy) step $\Delta$ by simple electrostatics, i.e., $\Delta=-e^{2} \int z \Delta n(z) d z / \epsilon_{0}$. 
The calculated electronic displacement without an external field, $\Delta n_{\mathrm{M} \mid \mathrm{BN}}\left(z, E_{\mathrm{ext}}=\right.$ $0)$, is shown in Fig. 5.6 for four different metal $\mid h$-BN interfaces. The amplitude of $\Delta n_{\mathrm{M} \mid \mathrm{BN}}(z)$ is seen to decrease with increasing separation of the adsorbed $h$-BN layer. The oscillation patterns illustrate the two different kinds of behavior discussed above. For $h$-BN adsorbed on the $\mathrm{Cu}(111)$ and $\mathrm{Au}(111)$ surfaces, the pattern of $\Delta n_{\mathrm{M} \mid \mathrm{BN}}(z)$ in the region between the metal surface and the $h$-BN layer is that of a simple dipole. It corresponds to an accumulation of electrons close to the metal surface and a depletion close to the $h$-BN layer, consistent with the pillow effect discussed above. The simple interface dipole and the large metal $\mid h-\mathrm{BN}$ equilibrium separations (Table 5.1) found for these systems are in line with physisorption of $h$-BN on these metals. In contrast, the oscillation pattern of $\Delta n_{\mathrm{M} \mid \mathrm{BN}}(z)$ for $h$-BN adsorbed on $\mathrm{Ni}(111)$ and $\mathrm{Pd}(111)$ surfaces is much more complicated. It is accompanied by a small metal- $h$-BN equilibrium distance, which is consistent with chemisorption.

In the model described by Eqs. (5.6)-(5.8), it was assumed that the interface potential steps $\Delta_{\mathrm{M} \mid \mathrm{D}}$ and $\Delta_{\mathrm{D} \mid \mathrm{Gr}}$ are independent of an applied external field. That implies that the corresponding interface dipoles should be independent of the external field. Figure 5.7 shows $\Delta n_{\mathrm{Cu} \mid \mathrm{BN}}\left(z, E_{\text {ext }}\right)$ for $h$-BN adsorbed on the $\mathrm{Cu}(111)$ surface calculated with $E_{\text {ext }}=-0.44,0$ and $+0.44 \mathrm{~V} / \AA$. This figure clearly demonstrates that $\Delta n_{\mathrm{Cu} \mid \mathrm{BN}}\left(z, E_{\text {ext }}\right)$ is essentially independent of the electric field strength within this range, and therefore also the interface dipole and the potential step. A similar independence can be observed in the electronic displacement at a $h$-BN|graphene interface in Fig. 5.8 (solid lines). Hence the interface dipoles, and the potential steps

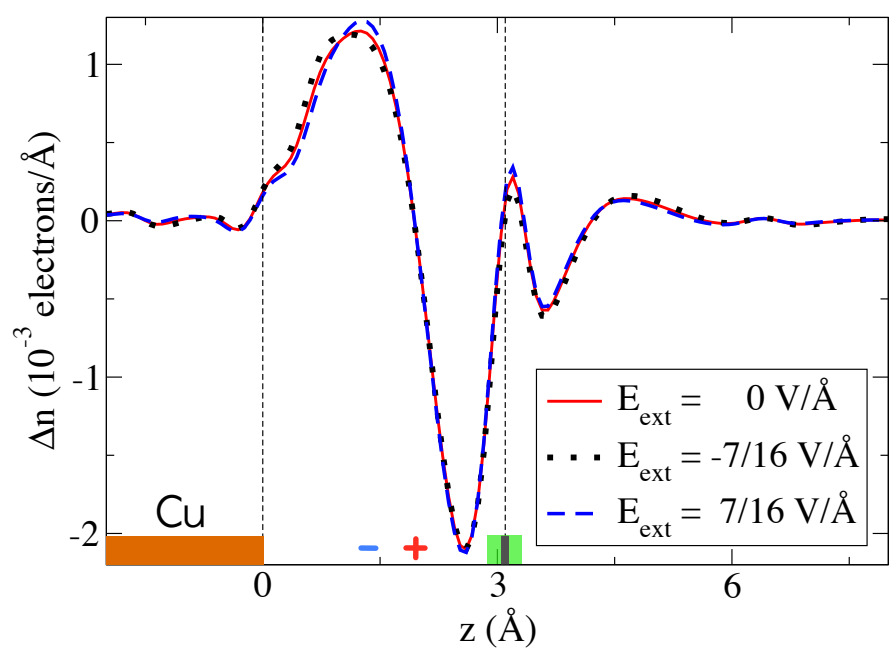

Figure 5.7: Influence of an external electric field on the $\mathrm{Cu}(111) \mid \mathrm{BN}$ interface dipole layer. The plane-averaged electron density difference $\Delta n_{\mathrm{Cu} \mid \mathrm{BN}}\left(z, E_{\text {ext }}\right)$ is shown for three different electric fields (perpendicular to the interface). The vertical dashed lines represent the positions of the atomic layers at the interface. 


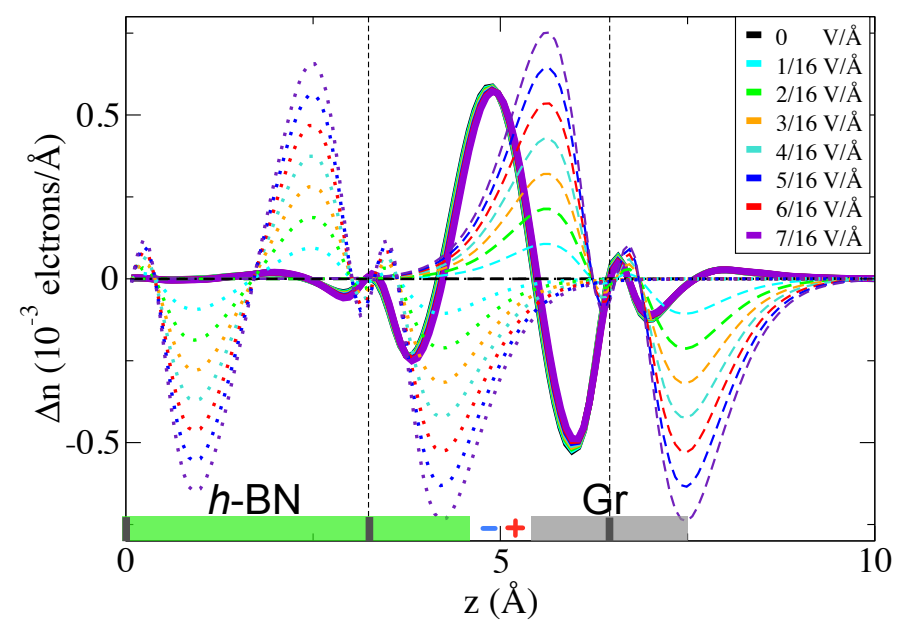

Figure 5.8: Influence of an external electric field on the $\mathrm{BN} \mid \mathrm{Gr}$ interface dipole layer. The plane-averaged electron density difference $\Delta n_{\mathrm{BN} \mid \mathrm{Gr}}\left(z, E_{\text {ext }}\right)$ is shown for different electric field strengths (solid lines). The dotted and dashed lines show the polarization of isolated $h$-BN and Gr sheets, respectively, in these external fields. The vertical dashed lines represent the positions of the atomic layers at the interfaces.

$\Delta_{\mathrm{M} \mid \mathrm{BN}}$ and $\Delta_{\mathrm{BN} \mid \mathrm{Gr}}$ are truly intrinsic properties of these interfaces.

The fact that the electronic displacement at the interfaces is field-independent (for the field strengths used here) does not mean that the electron densities are so, as both $h$-BN and graphene are polarized in an electric field. Fig. 5.8 shows the changes in electron density $n\left(z, E_{\text {ext }}\right)-n(z, 0)$ upon applying an electric field, both for an isolated graphene layer (dashed lines) and for an isolated $h$-BN slab (dotted lines). The changes clearly illustrate the induced dipoles on the individual layers.

For a complete metal $\mid h$-BN|graphene stack, we visualize the total electronic displacement caused by interface formation, as well as by the external electric field, as

$$
\Delta n_{\text {tot }}\left(z, E_{\text {ext }}\right)=n_{\mathrm{M}|\mathrm{BN}| \mathrm{Gr}}\left(z, E_{\mathrm{ext}}\right)-n_{\mathrm{M}}(z)-n_{\mathrm{BN}}(z)-n_{\mathrm{Gr}}(z),
$$

where the reference charge densities $n_{\mathrm{M}}, n_{\mathrm{BN}}, n_{\mathrm{Gr}}$ are for the isolated systems in zero external field. Results are shown in Fig. 5.9 for a $\mathrm{Cu}(111) \mid h$-BN $\mid$ graphene structure with five layers of $h$-BN. The dipoles at the $\mathrm{Cu}(111) \mid h$-BN and $h$-BN|graphene interfaces are essentially the same as the dipoles at individual isolated interfaces, see Figs. 5.7 and 5.8, implying that the two interfaces in the $\mathrm{Cu}(111) \mid h$-BN $\mid \mathrm{Gr}$ stack are not coupled.

$\Delta n_{\text {tot }}$ in Fig. 5.9 for the zero external field case $\left(E_{\text {ext }}=0\right)$ shows small oscillations in the $h$-BN slab, indicating that the $h$-BN layers are polarized. The effective work function difference $V_{0}$ between the $\mathrm{Cu}$ substrate and graphene, Eq. (5.8), results in electron transfer from $\mathrm{Cu}$ to graphene across the $h$-BN slab to establish a single Fermi 


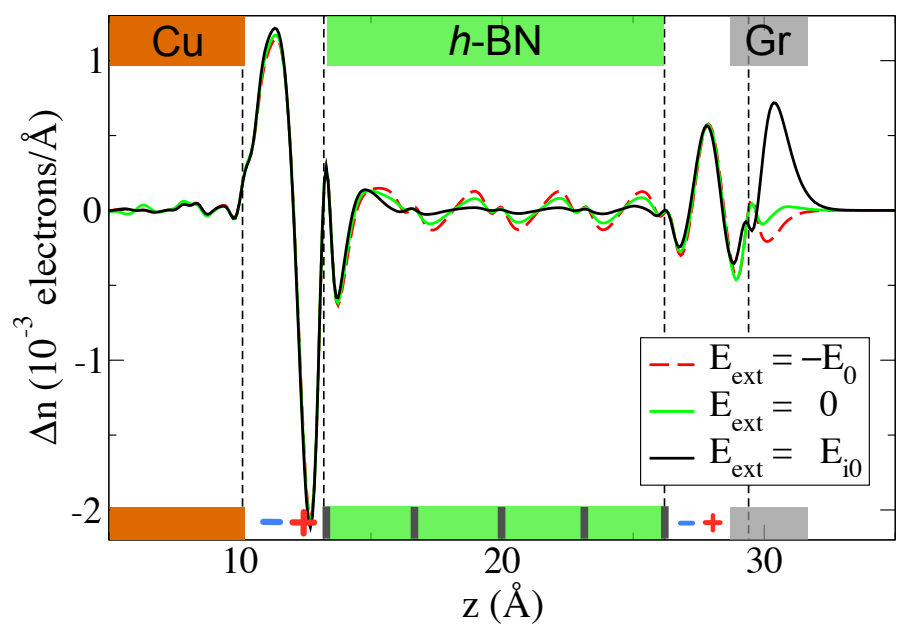

Figure 5.9: Plane-averaged electron density difference $\Delta n_{\text {tot }}\left(z, E_{\text {ext }}\right)$ for a $\mathrm{Cu}(111) \mid 5-$ layers-of- $h$-BN $\mid$ graphene structure for external electric fields $E_{\text {ext }}=-E_{0}, 0, E_{\mathrm{i} 0} \mathrm{~V} / \AA$. The vertical dashed lines represent the positions of the atomic layers at the interfaces.

level in equilibrium. This sets up an intrinsic electric field in the $h$-BN slab, see Eq. (5.7). This charge transfer can be counteracted by applying an external electric field $E_{\text {ext }}=-E_{0}$, see Eq. (5.6).

This external field results in charge neutral (i.e. undoped) graphene, but the field in the $h$-BN slab is still non-zero, see Eq. (5.4) with $\sigma=0$ and $E_{\text {ext }}=-E_{0}$. An external field of $E_{\text {ext }}=E_{\mathrm{i} 0} \approx-0.43 \mathrm{~V} / \AA$, Eq. (5.9), gives a zero internal field, see Fig. 5.9. Note that, whereas $\Delta n_{\text {tot }}$ in the region between the topmost $h$-BN layer and the graphene sheet is almost field-independent, it strongly depends on the field on the graphene side ( $z \gtrsim 30 \AA$ ). This just reflects the polarization behavior of the isolated graphene sheet, see Fig. 5.8.

\subsubsection{Fermi level shifts in metal $|h-\mathrm{BN}|$ graphene heterostructures}

All the parameters that appear in Eqs. (5.6-5.8) have now been calculated by studying constituent parts of $\mathrm{M} \mid h$-BN $\mid$ Gr stacks, Table 5.1, and the model can be tested by comparing its predictions to the results obtained from explicit DFT calculations on full $\mathrm{M} \mid h$-BN $\mid$ Gr structures. Fig. 5.5 demonstrates that the electric field dependence of the Fermi level shift is described very well by the model. We would like to emphasize that these curves do not represent data fits, as all parameters in the model are fixed.

We next study how the thickness of $h$-BN, measured in terms of the number of discrete layers $n$, influences the Fermi level shift $\Delta E_{\mathrm{F}}$ in graphene. In Table 5.2, $\Delta E_{\mathrm{F}}$ calculated with DFT for $\mathrm{Cu}(111) \mid n \times h$-BN $\mid$ graphene structures with $0<n<6$ layers is compared to values predicted by the model for a vanishing external field, $\Delta E_{\mathrm{F}}^{\mathrm{m}}$. The agreement between the two sets of results is to within $12 \mathrm{meV}$ or better. The 


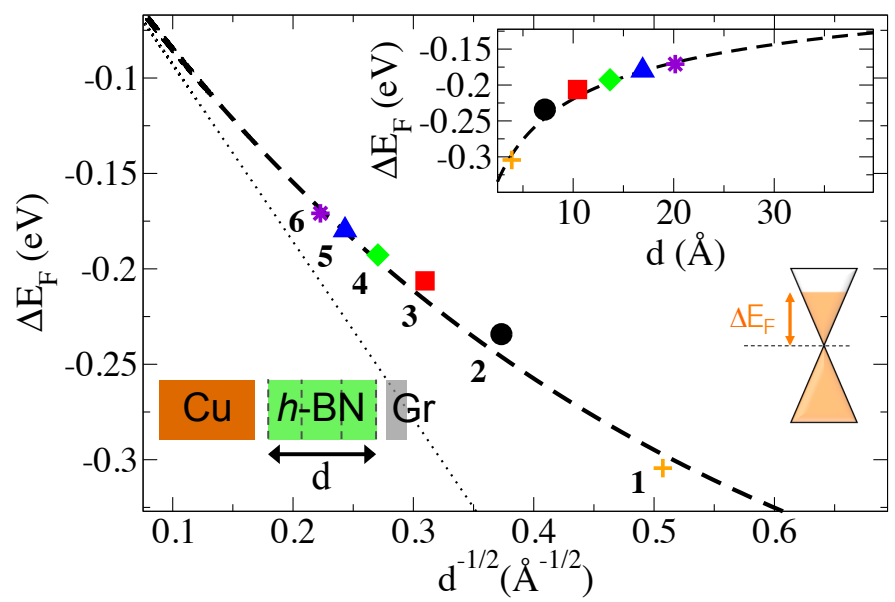

Figure 5.10: The Fermi level shift versus the thickness $d$ of the $h$-BN film, plotted as a function of $1 / \sqrt{d}$ and (inset) as a function of $d$. The symbols are the results of DFT calculations on $\mathrm{Cu}(111) \mid h$-BN $\mid$ graphene structures with 1 - 6 layers of $h$-BN. The dashed line represents the model calculated with Eq. (5.6), the dotted line represents the asymptotic $1 / \sqrt{d}$ dependence.

$n=0$ case corresponds to graphene directly adsorbed onto the $\mathrm{Cu}(111)$ surface and the results agree with those found previously.[72, 73] The good agreement found in Table 5.2 indicates that the two interfaces are not strongly coupled. Even for a single $h$-BN layer, $n=1$, the model works well.

In the absence of an applied potential, graphene in a $\mathrm{Cu}(111)|h-\mathrm{BN}|$ graphene stack is doped $n$-type in equilibrium. The degree of doping decreases as the thickness of the dielectric layer is increased. This can be simply understood from Eq. (5.6) where for large values of $d$, the Fermi level shift scales asymptotically with $d$ as $\Delta E_{\mathrm{F}} \propto 1 / \sqrt{d}$. Fig. 5.10 illustrates this scaling, and also demonstrates the deviation of this scaling for small $d$. The good agreement between the results of the first-principles calculations and the model seems to improve with increasing $h$-BN

Table 5.2: Calculated work function $W$ and Fermi energy shift $\Delta E_{\mathrm{F}}$ of $\mathrm{Cu}(111) \mid n \times h$ $\mathrm{BN} \mid$ graphene structures with $n$ layers of $h-\mathrm{BN}$, and Fermi energy shifts $\Delta E_{\mathrm{F}}^{\mathrm{m}}$ predicted by the model. All values are in $\mathrm{eV}$.

\begin{tabular}{lccccccc}
\hline$n$ & 0 & 1 & 2 & 3 & 4 & 5 & 6 \\
\hline$W$ & 4.386 & 4.223 & 4.299 & 4.325 & 4.336 & 4.346 & 4.354 \\
$\Delta E_{\mathrm{F}}$ & -0.171 & -0.304 & -0.234 & -0.206 & -0.193 & -0.180 & -0.171 \\
$\Delta E_{\mathrm{F}}^{\mathrm{m}}$ & $-0.17^{\mathrm{a}}$ & -0.297 & -0.246 & -0.216 & -0.196 & -0.181 & -0.169 \\
\hline
\end{tabular}

a Ref. [73] 


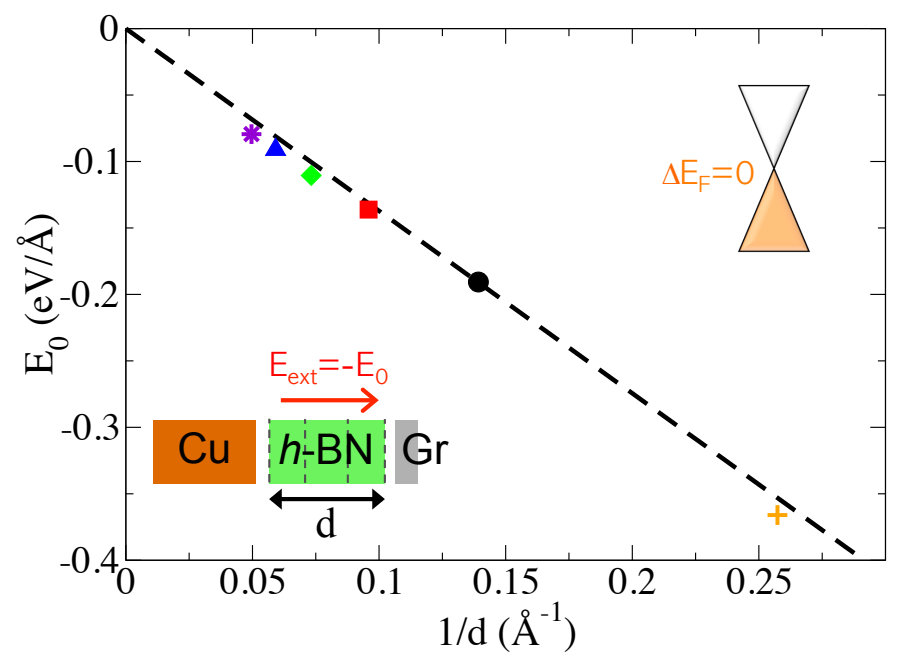

Figure 5.11: The value of the intrinsic electric field $E_{0}$ (Eq. (5.7)) as a function of $1 / d$ where $d$ is the thickness of the $h$-BN film. An external electric field $E_{\text {ext }}^{0}=-E_{0}$ is required to restore graphene to charge neutrality. The symbols are the results of DFT calculations for $\mathrm{Cu}(111) \mid h$-BN $\mid$ graphene structures with 1 - 6 layers of $h$-BN.

\section{thickness.}

As discussed in the previous section, the Fermi level can be positioned at the charge neutrality point of graphene by application of an external electric field $E_{\text {ext }}=$ $-E_{0}$. According to Eq. (5.7), $E_{0}$ should scale with the $h$-BN layer thickness $d$ as $E_{0} \propto 1 / d$. This scaling is demonstrated in Fig. 5.11. The symbols represent the $E_{\text {ext }}$ values needed in the DFT calculations to make graphene charge neutral in stacks with variable $h$-BN thickness. From Eq. (5.11) we then predict that the gate voltage required to shift the Fermi level to the graphene conical points should be independent of how thick $h$-BN is.

The dependence of the doping level, both as a function of the external field $E_{\text {ext }}$, and as a function of the $h$-BN layer thickness, is shown in Fig. 5.12. The agreement between the model and the DFT results is very good. The characteristic shape of the curves is determined by Eq. (5.6). The curves for different $h$-BN layer thicknesses cross at $E_{\text {ext }} \approx-0.4 \mathrm{~V} / \AA$. At this external field strength the doping level is independent of the thickness of the $h$-BN dielectric spacer. This point corresponds to the field $E_{\mathrm{i} 0}=-0.43 \mathrm{~V} / \AA$ discussed above, where the internal field in the $h$-BN spacer is zero.

For a dielectric consisting of two $h$-BN monolayers, Figure 5.13 shows how graphene in a metal $\mid h$-BN $\mid$ graphene structure is doped in the absence of an external field by different metals. Different metals have different workfunctions $W_{\mathrm{M}}$ and form different interface dipoles with the dielectric; each metal can be characterized by a different value of $V_{0}$ given by Eq. (5.8) using the parameters given in Table 5.1. The dashed 


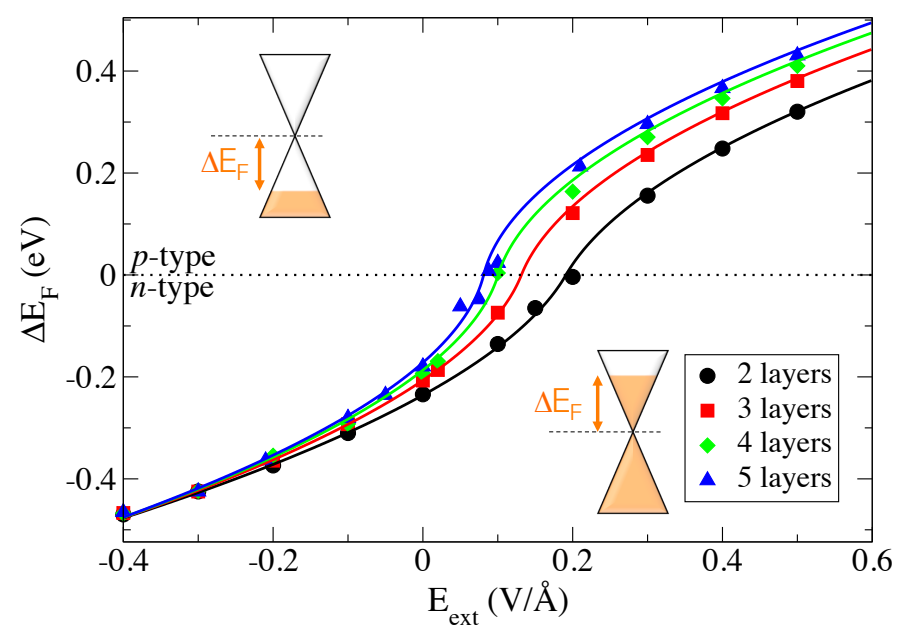

Figure 5.12: The Fermi level shift versus external electric field strength for a $\mathrm{Cu}(111)|h-\mathrm{BN}|$ graphene structure with 2 - 5 layers of $h$-BN. The lines represent the model of Eq.(5.6) for different thicknesses of dielectric. The symbols are values of the doping level calculated with DFT.

line represents the model of Eq. (5.12) for a fixed distance $d$, the circles correspond to the different metals where the equilibrium distances of Table 5.1 have been used. The predicted Fermi level shift $\Delta E_{\mathrm{F}}$ varies with the metal used for the gate electrode from $+0.18 \mathrm{eV}$ for $\mathrm{Pt}$ to $-0.39 \mathrm{eV}$ for Co.

Most of the metals considered give rise to $n$-type doping of graphene; $p$-type doping is only found for the high work function metals Au and Pt. The amount of doping is however much less than what would be expected on the basis of the work function difference between these metals and graphene only. This is because of the large potential step $\Delta_{\mathrm{M} \mid \mathrm{BN}}$ between $\mathrm{Au}$ or Pt and $h-\mathrm{BN}$, see Table 5.1, which effectively lowers the metal work function. Although most of the other metals have a work function that is comparable to or higher than that of graphene, they result in $n$-type and not in $p$-type doping. Again this can be attributed to the effect of the potential step $\Delta_{\mathrm{M} \mid \mathrm{BN}}$. A similar effect was found for graphene that is directly adsorbed on a metal substrate.[72, 73]

An external electric field can be used to counteract the intrinsic doping of graphene in these metal $\mid h$-BN|graphene stacks and position the Fermi level at the charge neutrality point of graphene corresponding to $\Delta E_{\mathrm{F}}=0$. The field strengths required for the different metals cover a range of $\sim 0.5 \mathrm{~V} / \AA$. For the stacks considered in Fig. 5.13, this corresponds to a range of $2 \mathrm{~V}$ in the gate voltage. 


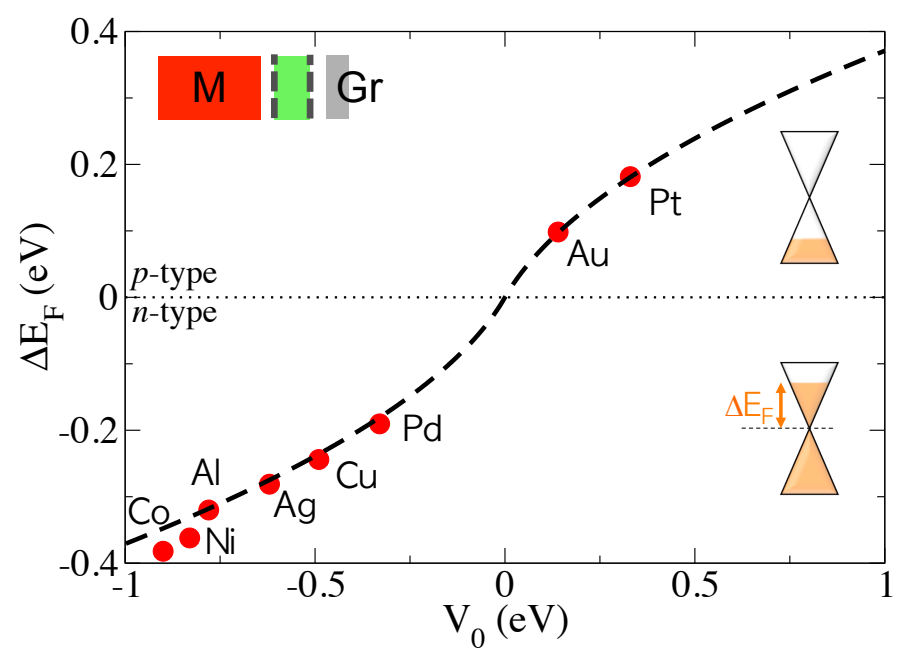

Figure 5.13: The Fermi level shift for metal $\mid h$-BN $\mid$ graphene structures with 2 layers of $h$-BN, plotted against $V_{0}$, according to Eqs. (5.12) and (5.8) with $V_{\mathrm{g}}=0$ and parameters from Table 5.1. The line is for a fixed $d$, and the circles are for the equilibrium metal $\mid h$-BN bonding distances.

\subsection{Summary and conclusions}

We have developed a model, Eq. (5.6), for the electrostatic doping of graphene in a field-effect device that describes the position of the Fermi level in graphene for a metal|dielectric|graphene structure as a function of the gate voltage. As input parameters the model needs the work function of the metal, the thickness and the dielectric constant of the dielectric material, and the potential steps formed at the metal|dielectric and the dielectric|graphene interfaces. All of these parameters can be obtained from DFT calculations on single surfaces or interfaces, which makes the model parameter-free.

The model predicts doping of graphene even when the bias voltage is zero. This effect is caused by charge equilibration, i.e. the need to establish a common Fermi level across the metal|dielectric|graphene stack. The amount of doping is not only guided by the difference in work function between the metal and graphene, but also to a significant extent by the potential steps at the interfaces. These potential steps result from interface dipole layers that are formed by the chemical interactions at the interface. In particular the potential step at the metal|dielectric interface can be large, i.e., $>1 \mathrm{eV}$, even when the bonding between the metal and the dielectric material is not strong. This potential step effectively lowers the work function of the metal, which causes metal substrates with work functions $\lesssim 5 \mathrm{eV}$ to dope graphene $n$-type. Only higher work function metal substrates lead to $p$-type doping.

The amount of doping at zero bias decreases with increasing thickness of the dielectric. The Fermi level shift in graphene as a function of the applied electric field 
resulting from the gate voltage has a square-root like behavior. A similar behavior has been observed in experiments on gated $p-\mathrm{Si}_{\mid}\left|\mathrm{SiO}_{2}\right|$ graphene structures, by work function measurements,[70] and by conductivity measurements.[69] According to our model, this square-root like behavior is a direct result of the density of states in graphene being linear around the conical points and implies that the adsorption of graphene on the dielectric in those experiments was sufficiently weak as to leave the electronic structure of graphene essentially unchanged.

The model is tested by comparing its predictions to results obtained from explicit DFT calculations on metal|dielectric|graphene structures in the presence of an external electric field. We use the (111) or (0001) surfaces of the fcc or hcp close-packed metals $\mathrm{Al}, \mathrm{Co}, \mathrm{Ni}, \mathrm{Cu}, \mathrm{Pd}, \mathrm{Ag}, \mathrm{Pt}, \mathrm{Au}$, which span a range of work functions from 4.2 to $6.0 \mathrm{eV}$. As our dielectric we use either vacuum or $h-\mathrm{BN}$, which is an insulating material with a similar honeycomb structure as graphene, and we vary the thickness of the dielectric by varying the number of $h$-BN layers. The model gives a good description of the Fermi level shift in graphene as a function of the applied electric field even for a $h$-BN dielectric only a monolayer thick. The thickness dependence of the Fermi level shift is also reproduced very well by the model.

To be able to perform microscopic DFT calculations it was necessary to make approximations which are in general not satisfied by real materials. In particular, we assumed that graphene is commensurate with $h$-BN and that both are commensurate with the underlying metal substrate. Where the in-plane bonding is very strong and the inter-plane bonding is weak as is the case for graphene on $h-\mathrm{BN}$, the resulting composite system remains incommensurate $[62,63,94,97]$ and it is not possible to carry out first-principles electronic structure calculations. Even when the bonding of graphene or $h$-BN to a metal substrate is stronger, the periods that are found for commensurability are frequently so large [103] that DFT calculations are exorbitantly expensive. In such cases, we suggest that the parameters in the model we have outlined should be regarded as free parameters and determined by fitting to experimental observations. In this way, it should be possible to apply the model to very much more complex systems such as $\mathrm{SiO}_{2}$ or $\mathrm{SiC}$ dielectrics and polycrystalline $\mathrm{Si}$ gates. Intrinsically planar dielectric spacers like $\mathrm{MoS}_{2}$ and $\mathrm{WS}_{2}$ that are currently of interest because of their potential in graphene electronics[80, 104] are intermediate; they can be studied using DFT calculations, modelling the interfaces with metals and graphene using large lateral supercells, or the relevant parameters can be determined by fitting to experiment, or a combination of both. 


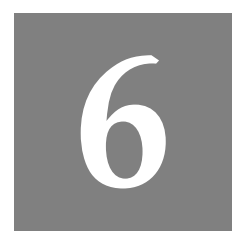

\section{Large potential steps at weakly interacting metal|insulator interfaces}

Potential steps exceeding $1 \mathrm{eV}$ can be formed at metallinsulator interfaces, even if the interaction between the materials at the interface is a weak van der Waals bonding. From density functional theory calculations on metal $\mid h-B N$ interfaces we show that the distance dependence of such potential steps is not sensitive to van der Waals interactions, although including these interactions leads to markedly different binding energy curves. We model the exchange repulsion using a symmetrized product of metal and $h$-BN wave functions, and demonstrate that exchange repulsion forms the main contribution to the interface potential step in the weakly interacting regime. Chemical interactions reduce the interface potential step in the strongly interacting regime.

\subsection{Introduction}

The formation of an interface between metals and materials such as graphene or $h$-BN leads to a dipole layer originating from the interactions at the interface. The dipole layer results in a potential step across the interface, which is of paramount importance to charge injection barriers. Naively one would expect that if the interaction between the two materials at the interface is weak, the interface dipole and potential step will be small. It is then suprising to find that physisorption of graphene or $h$-BN on metal substrates, with an adsorption energy of a mere $\sim 0.05 \mathrm{eV} /$ atom, leads to substantial potential steps of $\sim 1 \mathrm{eV}[72,83]$.

One explanation for a large potential step is a transfer of electrons across the interface. An electron transfer occurs between two conductors to equilibrate the chemical potential. This happens at metal|graphene interfaces, and results in a doping of 
graphene [72]. However, even for metal|graphene a large contribution to the potential step arises from a local interaction between graphene and the metal at the interface [73]. This contribution depends roughly exponentially on the distance between graphene and the metal surface, stressing its local character. An electron transfer does not occur across a metal|insulator interface, such as metal $\mid h-\mathrm{BN}$. There the full potential step originates from the interactions at the interface [105].

In this paper we explore the origins of the interface dipole and potential step at metal|insulator interfaces by density functional theory (DFT) calculations. We focus on metal $\mid h$-BN as an archetypal metal|insulator interface with interactions varying from weak (physisorption) to strong (chemisorption), depending on the metal. As a function of the metal- $h$-BN distance the interface dipole is roughly exponential, similar to metal|graphene.

Terms with an exponential distance dependence also contribute to dipoles created by interactions between (closed-shell) molecules or ions [106]. Such terms are ascribed to Pauli exchange repulsion, which pushes electrons out of the overlap region between molecules, resulting in a distortion of the electron distribution [107]. The adsorption of chemically inert atoms or molecules on metal surfaces also leads to dipoles attributed to exchange repulsion. This phenomenon is colloquially called the pillow effect or push-back effect [108, 96, 109, 110, 111].

We construct a quantitative model of the effect of exchange repulsion on the interface potential step, derived from an antisymmetrized product of the wave functions of the two materials at the interface. Comparing the potential steps calculated with this model to those obtained from self-consistent DFT calculations, we establish that exchange repulsion forms the main contribution to the large potentials steps found for $h$-BN physisorbed on a metal substrate. A smaller but significant contribution is given by a dipole that originates from bonding between the two materials, e.g., by van der Waals (vdW) interactions.

\subsection{DFT calculations}

The potential step $\Delta V_{S C F}$ at a metal|insulator interface $\mathrm{A} \mid \mathrm{B}$ can be obtained from self-consistent calculations as the difference between the work functions of the clean metal surface $\mathrm{A}$ and that of the combined system, $\Delta V_{S C F}=W_{A}-W_{A \mid B}$. Here we use as metal A the closed-packed (111) or (0001) surfaces of the metals $\mathrm{Co}, \mathrm{Cu}$, $\mathrm{Ag}, \mathrm{Au}, \mathrm{Pd}$ and $\mathrm{Pt}$, and as insulator $\mathrm{B}$ a $h$-BN monolayer. We consider commensurate interfaces, accomodating lattice mismatches by adapting the in-plane lattice constant of the metals to that of $h$-BN. Changing the lattice constant of a metal by a few percent changes its properties only mildly, whereas adapting the lattice constant of $h-\mathrm{BN}$ is a much larger perturbation.

For the DFT calculations we use the Vienna Ab initio Simulation Package (VASP) [44, 45, 12], and follow Ref. [105] concerning interface structures and settings of computational parameters. We consider three different functionals: the local den- 
sity approximation (LDA) [16] , the PBE generalized gradient approximation (GGAPBE) [20], and the optB88-vdW-DF van der Waals density functional [23, 112, 113]. LDA gives a reasonable description of the binding energy and geometry at a metal $\mid h$ $\mathrm{BN}$ interface, although it is known to overestimate chemical interactions in general. GGA usually gives a good description of chemisorption, but fails to capture physisorption. (Semi-)local functionals lack vdW interactions, which play an important role in physisorption. These interactions are modeled in non-local vdW functionals. We use the optB88-vdW-DF functional, which gives a good description of the the bonding between the graphene layers in graphite, for instance.

Figure 6.1(a) shows the binding energy curves of $h-\mathrm{BN}$ on $\mathrm{Cu}(111)$ for the three functionals. GGA gives almost no bonding, with an adsorption energy $E_{b}=-1$ $\mathrm{meV} / \mathrm{BN}$ at an equilibrium distance $d_{e q}=4.1 \AA$, whereas LDA gives a reasonable bonding, with $E_{b}=-87 \mathrm{meV} / \mathrm{BN}$ at $d_{e q}=3.0 \AA$. These findings are consistent with physisorption. Indeed optB88-vdW-DF gives $E_{b}=-140 \mathrm{meV} / \mathrm{BN}$ at $d_{e q}=3.3 \AA$, indicating the importance of $\mathrm{vdW}$ interactions [114, 115].

Whereas the binding energy curves depend rather sensitively on the type of functional used, the interface potential step is remarkably impervious to the functional. This is demonstrated in Fig. 6.1(b), which shows the potential step $\Delta V_{S C F}$ at the $\mathrm{Cu}(111) \mid h$-BN interface as a function of the distance $d$ between the $\mathrm{Cu}(111)$ surface and the $h$-BN plane. The curves for the three functionals are within $0.05 \mathrm{eV}$ of one another. The potential step is proportional to the interface dipole, which can be derived from the electron displacement $\Delta n_{S C F}=n_{A \mid B}-n_{A}-n_{B}$, where $n_{A \mid B}, n_{A}, n_{B}$ are the charge densities of the metal $\mid h-\mathrm{BN}$, and the isolated metal and $h$-BN systems, respectively.

The insensitivity of the potential step to the functional suggests a similar insensitivity of the electron displacement $\Delta n_{S C F}$, which is confirmed by Figs. 6.1(c) and (d). At $d=3 \AA$, which is close to the equilibrium distance, $\Delta V_{S C F} \approx 1 \mathrm{eV}$, and $\Delta n_{S C F}$ is very similar for all three functionals. At a larger distance, $d=5 \AA, \Delta n_{S C F}$ of the vdW-DF functional shows an accumulation of electrons in the space between the top metal and the $h$-BN planes, and a depletion close to these planes. Such a pattern is also observed in a $(\mathrm{Ar})_{2} \mathrm{vdW}$ complex, suggesting that this is typical for $\mathrm{vdW}$ interactions [112]. Indeed this pattern is effectively absent from the PBE $\Delta n_{S C F}$ at $d=5 \AA$. Interestingly, LDA shows a $\Delta n_{S C F}$ that is quite similar to that the vdW-DF. A local functional of course does not represent vdW interactions properly, which is why the binding energy curves are different. Nevertheless, both LDA and vdW-DF give qualitatively similar charge distributions.

$\Delta V_{S C F}<0.05 \mathrm{eV}$ at $d=5 \AA$, indicating that $\mathrm{vdW}$ interactions as such do not give a large interface dipole. Moreover, the insensitivity of the dipole to the DFT functional suggests a model that does not rely upon a specific functional. 

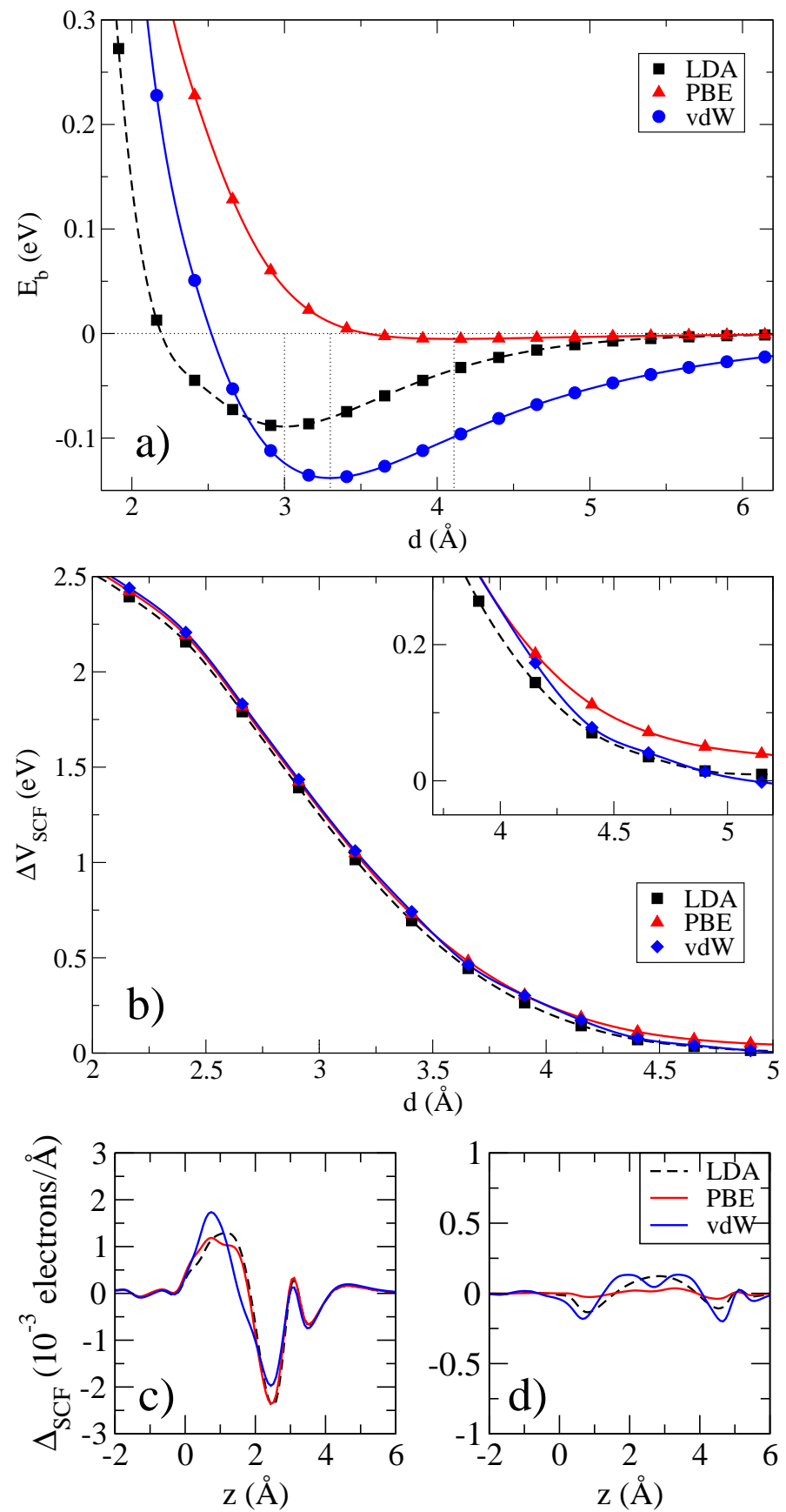

Figure 6.1: (a) Binding energy curves $E_{b}(d)(\mathrm{eV} / \mathrm{BN})$ of $h$ - $\mathrm{BN}$ on $\mathrm{Cu}(111)$, calculated with LDA (black squares), GGA-PBE (red triangles), and optB88-vdW-DF(blue circles) functionals. The vertical lines indicate the minima. (b) The interface potential steps $\Delta V_{S C F}(d)$ calculated with the three functionals. (c,d) The plane averaged electron displacements $\Delta \bar{n}_{S C F}$ at $d=3$ and $5 \AA$. 


\subsection{Model}

An approximation for the ground state of an interface $\mathrm{A} \mid \mathrm{B}$ should at least be a fermionic state. If we start from two well-separated systems A and B, then a simple expression of such a state is given by a Heitler-London like expression

$$
|\Psi\rangle=\hat{A} \hat{B}|0\rangle
$$

where

$$
\hat{X}=\prod_{\mathbf{k} n ; \text { occ }} \hat{c}_{X, \mathbf{k} n}^{\dagger} ; X=A, B
$$

Here $|0\rangle$ is the the vacuum state, $\mathbf{k} n$ the Bloch vector and band index, and $X$ the system. The fermion operator $\hat{c}_{X, \mathbf{k} n}^{\dagger}$ creates an electron in the orbital $\left|\phi_{\mathbf{k} i}^{X}\right\rangle$, and the product is over all occupied states. The state $|\Psi\rangle$ incorporates the exchange of electrons among any of the occupied orbitals of A and B. As such it defines the Pauli exchange interaction between systems $\mathrm{A}$ and $\mathrm{B}$. We refer to this state as the antisymmetrized (AS) product state.

If the orbitals on A and B overlap at the inteface between the two systems, they are in general not orthogonal. The technical difficulties of calculating expectation values with non-orthogonal orbitals can be circumvented by a simple procedure. Start with a linear transformation, $\hat{c}_{\beta}^{\dagger}=\sum_{\alpha} \hat{c}_{\alpha}^{\dagger} T_{\alpha \beta}$, where $\alpha$ or $\beta$ is a short-hand notation for the combined index $(X, \mathbf{k} n)$, and it runs over all occupied states of both systems $\mathrm{A}$ and $\mathrm{B}$. The same transformation defines new orbitals, $\left|\phi_{\beta}^{\prime}\right\rangle=\sum_{\beta}\left|\phi_{\alpha}\right\rangle T_{\alpha \beta}$. The state $|\Psi\rangle$ is invariant under such a transformation, apart from a multiplicative factor, $\left|\Psi^{\prime}\right\rangle=\operatorname{det}(T)|\Psi\rangle$, which follows directly from its definition, Eqs. (6.1) and (6.2).

We then use a special transformation that orthogonalizes the orbitals, $\left\langle\phi_{\alpha}^{\prime} \mid \phi_{\beta}^{\prime}\right\rangle=$ $\delta_{\alpha \beta}=\sum_{\gamma, \zeta} T_{\gamma \alpha}^{*} S_{\gamma \zeta} T_{\zeta \beta}$, where $S_{\gamma \zeta}=\left\langle\phi_{\gamma} \mid \phi_{\zeta}\right\rangle$ is the overlap matrix of the original orbitals. In matrix form $\mathbf{I}=\mathbf{T}^{\dagger} \mathbf{S T}$, or $\mathbf{T} \mathbf{T}^{\dagger}=\mathbf{S}^{-1}$. The expectation value with respect to $|\Psi\rangle$ of any operator can now be calculated using the standard expressions for orthogonal orbitals. For instance, for any one-particle operator one obtains $\sum_{\alpha}\left\langle\phi_{\alpha}^{\prime}|\hat{o}| \phi_{\alpha}^{\prime}\right\rangle=\sum_{\alpha, \beta}\left\langle\phi_{\alpha}|\hat{o}| \phi_{\beta}\right\rangle S_{\beta \alpha}^{-1}$.

The density operator $\hat{n}(\mathbf{r})=|\mathbf{r}\rangle\langle\mathbf{r}|$ is an example of a one-particle operator, whose expectation value is the electron density

$$
n(\mathbf{r})=\sum_{\alpha, \beta} \phi_{\alpha}^{*}(\mathbf{r}) \phi_{\beta}(\mathbf{r}) S_{\beta \alpha}^{-1},
$$

with $\phi_{\alpha}(\mathbf{r}) \equiv\left\langle\mathbf{r} \mid \phi_{\alpha}\right\rangle$. We define the electron displacement $\Delta n(\mathbf{r})$ as the change in the electron density of the combined system $\mathrm{AB}$ with respect to the summed electron densities of the two separate systems A and B

$$
\Delta n(\mathbf{r})=\sum_{\alpha, \beta} \phi_{\alpha}^{*}(\mathbf{r}) \phi_{\beta}(\mathbf{r})\left(S_{\beta \alpha}^{-1}-\delta_{\beta \alpha}\right) .
$$




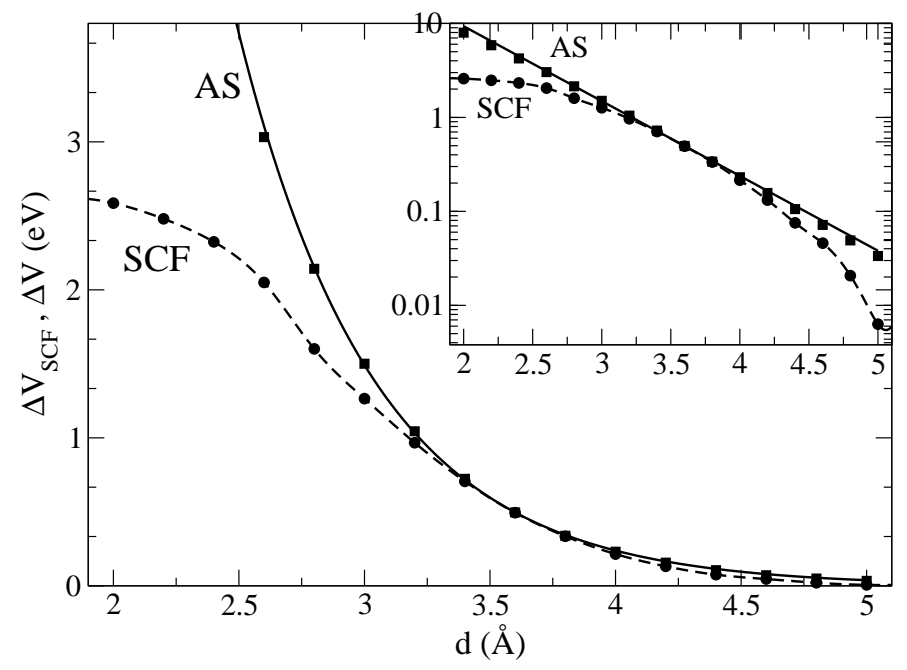

Figure 6.2: Potential steps $\Delta V_{S C F}, \Delta V$ at the $\mathrm{Cu}(111) \mid h$-BN interface from selfconsistent calculations and from the AS state, Eqs. (6.1)-(6.5), respectively. Inset: potential steps on a logarithmic scale.

The double sum is over all occupied orbitals $(X, \mathbf{k} n)$. The overlap matrix and its inverse are of course block-diagonal in $\mathbf{k}$, but not in the system and band indices $X, n$.

By construction $\int \Delta n(\mathbf{r}) d^{3} r=0$, if integrated over all space. If the overlap between the subsystems $\mathrm{A}$ and $\mathrm{B}$ is confined to an interface, then $\Delta n(\mathbf{r}) \rightarrow 0$ away from the interface. Solving the Poisson equation with $\Delta n(\mathbf{r})$ as source then gives a step in the potential (energy) across the interface

$$
\Delta V=\frac{e^{2}}{\epsilon_{0}} \int_{-\infty}^{\infty} z \Delta \bar{n}(z) d z
$$

Here $z$ is the direction normal to the interface, $\Delta \bar{n}(z)=\frac{1}{A} \int_{s} \Delta n(\mathbf{r}) d^{2} r$ the plane averaged electron displacement and $-e$ times the integral on the rhs of Eq. (6.5) is the interface dipole surface density.

\section{4 $\mathrm{Cu}(111) \mid h-\mathrm{BN}$ interface}

Figure 6.2 shows the potential step $\Delta V$ at the $\mathrm{Cu}(111) \mid h$-BN interface as function of the distance $d$ between the $\mathrm{Cu}(111)$ surface and the $h$-BN plane, calculated with the AS state according to Eqs. (6.1)-(6.5). $\Delta V$ is an exponential function of $d$, which is consistent with the fact that it depends on the overlap between the $\mathrm{Cu}$ and the $h$-BN wave functions at the interface. The behavior of the potential step obtained from fully self-consistent calculations, $\Delta V_{S C F}$, is slightly more complicated. For a 

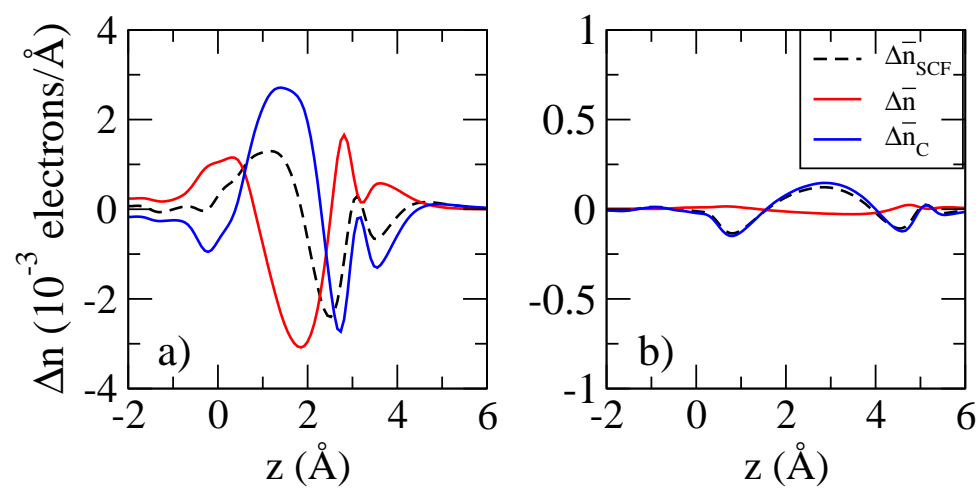

Figure 6.3: Plane averaged electron displacements $\Delta \bar{n}_{S C F}$ (black dashed) from selfconsistent calculations, $\Delta \bar{n}$ (red) from the AS state, Eq. (6.4), and $\Delta \bar{n}_{C}$ (blue) the bonding contribution, for a $\mathrm{Cu}(111)-h$-BN distance of (a) $3 \AA$, and (b) $5 \AA$.

distance $d \approx 3.5 \AA, \Delta V_{S C F}$ coincides with $\Delta V$. In this regime exchange repulsion between $\mathrm{Cu}(111)$ and $h$-BN provides a good description of the interface potential step.

For distances $d \lesssim 3.0 \AA \Delta V_{S C F}$ deviates from $\Delta V$. At these shorter distances stronger chemical interactions between the two systems become dominant, resulting in a more drastic reorganization of electrons, and in a deviation from a simple exponential curve. At the vdW-DF equilibrium distance $\left(d_{e q}=3.3 \AA\right)$, the potential step calculated from exchange repulsion is only $\sim 5 \%$ higher than the SCF value. At the LDA equilibrium distance $\left(d_{e q}=3.0 \AA\right)$, exchange repulsion overestimates the SCF value by $\sim 25 \%$. The SCF values at 3.0 and $3.3 \AA$ are 1.18 and $0.82 \mathrm{eV}$, respectively, in excellent agreement with the experimentally observed range $0.8-1.1 \mathrm{eV}$ [97].

For distances $d \gtrsim 4.0 \AA \Delta V_{S C F}$ also starts to deviate from $\Delta V$. This is caused by the long range vdW interaction between $\mathrm{Cu}(111)$ and $h$-BN as discussed above, see Fig. 6.1. Note however that at these distances $\Delta V_{S C F}<0.1 \mathrm{eV}$, so this effect is small.

The electron displacement $\Delta \bar{n}(z)$ associated with exchange repulsion at the interface is shown in Fig. 6.3. Exchange repulsion pushes electrons out of the overlap region between the $\mathrm{Cu}(111)$ surface and the $h$-BN plane. The system as a whole stays neutral, and the depleted electrons are accumulated close to the $\mathrm{Cu}(111)$ surface and the $h$-BN plane. The depletion/accumulation pattern is asymmetric. The wave functions of the $\mathrm{Cu}(111)$ surface extend more into the vacuum than the $h$-BN wave functions, implying that the overlap affects the former over a larger region than the latter, and that the effects of exchange repulsion are larger on the $\mathrm{Cu}(111)$ side than on the $h$-BN side.

The asymmetric depletion/accumulation pattern results in an net interface dipole that points out of the $\mathrm{Cu}(111)$ surface. Compared to the clean $\mathrm{Cu}(111)$ surface, adsorption of $h$-BN pushes back some of the electrons that otherwise spill into the vac- 
uum. The potential step $\Delta V$ is downwards going from $\mathrm{Cu}(111)$ to $h$-BN. In other words, exchange repulsion decreases the work function with respect to the clean metal. Such a decrease is commonly observed, not only in the physisorption of $h$-BN on other metal substrates (see below), but also in the physisorption of graphene, and of organic molecules.

The shape of the electron displacement $\Delta \bar{n}$ is fairly independent of the distance $d$ between the $\mathrm{Cu}(111)$ surface and the $h$-BN plane, but its amplitude decreases exponentially with increasing distance, consistent with it being caused by overlap, see Eq. (6.4). Although exchange repulsion accounts for most of the potential step at distances around the equilibrium distance, $\Delta \bar{n}$ is not identical to the self-consistent $\Delta \bar{n}_{S C F}$, see Figure 6.3. The difference $\Delta \bar{n}_{C}=\Delta \bar{n}_{S C F}-\Delta \bar{n}=\bar{n}_{S C F}-\bar{n}$ can be ascribed to chemical and vdW bonding.

Figure 6.3 shows $\Delta \bar{n}_{C}$ at a number of distances $d$. At all distances $\Delta \bar{n}_{C}$ shows an accumulation of electrons between the $\mathrm{Cu}(111)$ surface and the $h$-BN plane, accompanied by a depletion of electrons in the $\mathrm{Cu}(111)$ surface and the $h$-BN plane. Such a depletion/accumulation pattern is typical of bond formation. $\Delta \bar{n}_{C}$ changes both shape and amplitude as a function of $d$.

At short distances $d<3 \AA, \Delta \bar{n}_{C}$ gives a sizeable dipole opposite to that calculated from exchange repulsion. Interpreting $\Delta \bar{n}_{C}$ as bond formation, the polarity of the bond is then such that $h$ - $\mathrm{BN}$ is on the negative side, which is consistent with the fact that $h$-BN is more electronegative than $\mathrm{Cu}$. The result is that $\Delta V_{S C F}<\Delta V$.

Remarkably, at distances around the equilibrium distance $3.3 \AA, \Delta \bar{n}_{C}$ shows a pattern that is fairly symmetric with respect to $\mathrm{Cu}(111)$ and $h$-BN, such that the resulting dipole is moderate. So, although the electron displacement $\Delta \bar{n}$ associated with exchange repulsion deviates from the self-consistent $\Delta \bar{n}_{S C F}$, the interface potential step $\Delta V_{S C F} \approx \Delta V$. As $\Delta \bar{n}$ goes to zero exponentially as a function of $d, \Delta \bar{n}_{C}$ approaches $\Delta \bar{n}_{S C F}$ for large $d$. The electron displacement coming from the $\mathrm{vdW}$ bond is the only term remaining at these distances, but it results only in a small potential step.

\subsection{Interface potential steps}

Potential steps as function of $d$ at metal $\mid h$-BN interfaces are shown in Fig. 6.4 for six different metal substrates. At $d \approx 3.5 \AA$ the curves for the self-consistent potential steps $\Delta V_{S C F}$ converge with those of the exchange repulsion potential steps $\Delta V$. At such distances the exchange repulsion is the dominant cause of the interface potential steps. Although the electron displacement coming from the $\mathrm{vdW}$ bond has the longest range, it does not yield a sizeable potential step. At $d<3.0 \AA$ chemical interactions become important, and the contribution to the potential step of the electron displacement resulting from chemical bonds, is not negligible. As for $\mathrm{Cu}(111)$, this contribution tempers the potential steps as compared to exchange repulsion only.

The exchange repulsion potential steps have an exponential behavior $\Delta V(d) \approx$ 


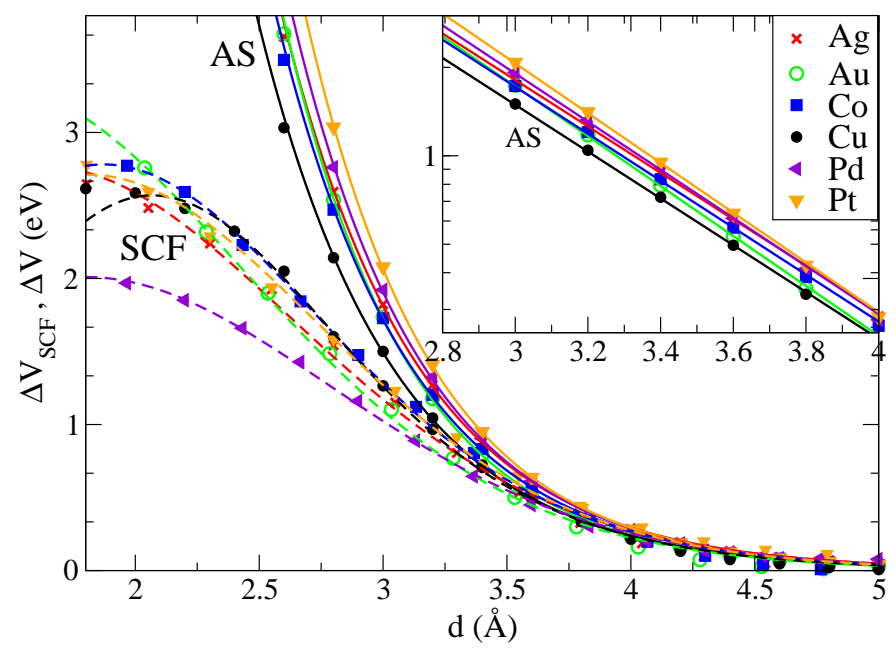

Figure 6.4: (a) Potential steps $\Delta V_{S C F}, \Delta V$ at metal $\mid h$-BN interfaces from selfconsistent calculations and the AS state, respectively. Inset: $\Delta V$ on a logarithmic scale.

$a_{0} e^{-\gamma d}$. There is a correlation between the exponent $\gamma$ and the work function $W$ of the metal, i.e., $\gamma$ increases if $W$ increases. The correlation is weak, however, with $\gamma$ varying between 1.82 for $\mathrm{Ag}$ and 1.95 for Pt. It is then not surprising that a single, mean $\gamma$ can be used to reasonable fit the curves for all metals.

As the exchange repulsion gives the largest contribution to the potential step, we can express the self-consistent potential step as $\Delta V_{S C F} \approx f(d) e^{-\gamma d}$, with $f(d)$ a simple function describing the deviation from exchange repulsion at distances much different from the equilibrium distance. In the adsorption of graphene on metal substrates, for instance, a second order polynomial has been used for $f(d)$ to describe the medium and large distance behavior $[72,73]$. The coefficient of the polynomial can be chosen independent of the metal. This also works for the adsorption of $h$-BN on metal surfaces. At short distances, where chemical bonding sets in, the $f(d)$ has to become metal-dependent, as is clear from Fig. 6.4.

\subsection{Summary}

We have studied the formation of potentials steps at metal|insulator interfaces, using metal $\mid h$-BN interfaces as archetypal example. Such potential steps can be surprisingly large, i.e., in excess of $1 \mathrm{eV}$, even though the bonding at the interface is a weak van der Waals bonding. Constructing an model for the Pauli exchange repulsion at the interface, we identify the contribution of the exchange repulsion to the interface potential step. At distances between the metal and the insulator surfaces that are typical for physisorption, exchange repulsion forms the main contribution to the 
interface potential step. At these and larger distances, chemical/van der Waals interactions significantly modify the local electron density, but give a relatively small contribution to the potentials step. At shorter distances chemical bonding interactions reduce the interface potential step. 


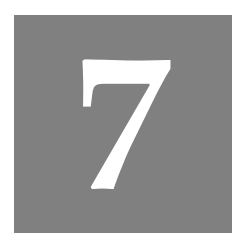

\section{Schottky barriers at metal|hexagonal boron nitride interfaces: a first principles study}

The Schottky barrier formation at metal|hexagonal boron nitride (h-BN) interfaces is studied by density functional theory calculations. For a range of metals with work functions between 4.2 and $6.0 \mathrm{eV}$, we find Schottky barriers for holes between 0.9 and $2.5 \mathrm{eV}$. A central role in fixing the Schottky barrier is played by a potential step between 0.4 and $1.8 \mathrm{eV}$, which is formed at the metal- $h-B N$ interface, and effectively lowers the metal work function. If $h$-BN is physisorbed, as is the case for $C u, A l, A u, A g$ and Pt(111) substrate the interface potentials step is described well by a universal function that depends on the distance between the metal surface and the $h-B N$ plane only. The interface potential step is largest if $h-B N$ is chemisorbed, as is the case for Co(0001), Ni and Pd(111) substrates.

\subsection{Introduction}

Hexagonal boron nitride ( $h$-BN) has a crystal lattice similar to that of graphite.The boron and nitrogen atoms within a single layer of $h$-BN form a strongly bonded hexagonal lattice analogous to that in graphene, and the bonding between the layers is weak, as in graphite. In contrast to graphene/graphite, $h$-BN is an insulator, making it a very suitable substrate and gate dielectric for applications in graphene electronics. $[64,4,61,62,63,83,68,116,105]$ Indeed it has been shown that the mobility of the charge carriers in graphene adsorbed on $h$ - $\mathrm{BN}$ is comparable to that in suspended graphene.[61, 62, 63] The high mobility has been attributed to the fact that the $h$-BN is atomically flat,[61] and gives a nearly homogeneous electrostatic 
potential in graphene.[63, 62]

Like graphene, $h$-BN layers can be prepared by mechanical exfoliation.[64] Cleaved layers can be thinned to a single layer with a high-energy electron beam.[65] Alternatively, $h$-BN layers can be grown by chemical vapor deposition (CVD) on transition metals such as $\mathrm{Cu}$ or $\mathrm{Ni}$, using precursors such as borazine $\left(\mathrm{B}_{3} \mathrm{~N}_{3} \mathrm{H}_{6}\right)$ or ammonia borane $\left(\mathrm{NH}_{3}-\mathrm{BH}_{3}\right)$. $[66,67]$ With a proper choice of growth conditions, homogeneous ultrathin $h$-BN layers can be grown of 1-5 monolayers. Graphene can be grown by CVD on top of a $h$-BN layer adsorbed on a metal substrate, $[68,116]$ which is ideal for field-effect devices.

Hexagonal boron nitride ( $h$-BN) is a wide band gap material, with a measured band gap of $5.97 \mathrm{eV} .[60]$ The direct nature of its band gap makes $h$-BN an interesting candidate for applications in ultraviolet light emitting diodes and lasers, but obviously the size of the band gap makes it difficult to design stable $p$ - $n$ junctions or ohmic metal contacts. It has been suggested that $h$-BN is a material with a negative electron affinity,[117] which would make obtaining a low barrier contact for electrons extremely challenging. At the same time this would increase the chances for making low barrier contacts for holes. Having low barrier metal contacts is advantageous for using $h$-BN as a semiconductor. It is detrimental for graphene devices, where $h$-BN is used as an insulator.

In this paper we determine the Schottky barrier for holes $\left(\Phi_{p}\right)$ between a metal contact and $h$-BN, for a series of metals with work functions ranging from 4.2 to 6.0 $\mathrm{eV}$, using density functional theory (DFT) calculations. Substantial Schottky barriers $\left(0.9<\Phi_{p}<2.5 \mathrm{eV}\right)$ are found in all cases. There is not a simple relation between the sizes of the Schottky barrier and the work function. A key role is played by a potential step at the metal $\mid h$-BN interface, which originates from an interface dipole layer formed upon adsorbing $h$-BN on a metal surface. This potential step effectively lowers the metal work function, and increases the Schottky barrier. For weak adsorption (physisorption) the size of the interface dipole can be described by a universal function that only depends on the metal $\mid h-\mathrm{BN}$ bonding distance, and not on the metal.

Special attention is given to the case where the adsorption energies are weak and a lattice mismatch between $h-\mathrm{BN}$ and the metal surface exists. Such conditions likely lead to a super-structure with a periodically modulated interface dipole and potential step that have the same periodicity as the super-structure.

\subsection{Method and Computational details}

We use density functional theory (DFT) to calculate ground state energies and optimized geometries, with a projector augmented wave (PAW) basis set,[14, 12] as implemented in VASP.[44, 45] The structures are modeled by a periodic slab. A dipole correction is applied to avoid spurious interactions between periodic images of the slab.[91] The plane wave kinetic energy cutoff is set at $400 \mathrm{eV}$. We apply a $24 \times 24 \mathbf{k}$ point grid to sample the surface Brillouin Zone (BZ) and use the tetrahedron scheme 
for BZ integrations. The electronic self-consistency criterion is set to $10^{-7} \mathrm{eV}$. Total energies with respect to geometry relaxation are converged to within $10^{-6} \mathrm{eV}$. The boron and nitrogen atoms in the $h$-BN slab, and the top two atomic layers of the metal surface are allowed to relax during the geometry optimization.

The interaction in weakly (van der Waals) bonded systems is difficult to describe within DFT. Commonly used generalized gradient approximation (GGA) functionals, such as PW91 or PBE, incorrectly predict essentially no bonding between $h$-BN or graphene layers, [118] as well as no bonding between $h$-BN or graphene and transition metal (111)-surfaces.[119, 73, 120] The local density approximation (LDA) gives a reasonable description of the bonding between $h$-BN layers (geometry and energy) and between a $h$-BN and a graphene layer, $[4,83,105]$ and of the bonding between $h$-BN or graphene and metal(111) surfaces.[72, 73, 105] In general, however, LDA is known to overestimate chemical bonding, and it does not capture van der Waals interactions properly.

In this paper we use (besides LDA) one of the recently developed and implemented van der Waals density functionals (vdW-DF), $[23,112,113]$ and compare the results to those obtained with LDA. In the vdW-DF the exchange-corrrelation functional is split up as $E_{\mathrm{xc}}=E_{\mathrm{x}}+E_{\mathrm{c}}^{\mathrm{vdW}}+E_{\mathrm{c}}^{\text {loc }}$, where $E_{\mathrm{c}}^{\mathrm{vdW}}$ describes the non-local electron-electron correlations, and $E_{\mathrm{c}}^{\text {loc }}$ describes the local correlations. For these we use the vdW kernel developed by Dion et al.,[23] and the LDA correlation, respectively. For the exchange part $E_{\mathrm{x}}$ we use the optB88 functional.[113] The optB88$\mathrm{vdW}$-DF functional gives a decent description of the lattice parameters and binding energy of graphite, as well as of the structures and energetics of Li intercalation in graphite.[121]

\subsection{Results}

\subsubsection{Metal $\mid h$-BN structures and bonding}

We have calculated the equilibrium structures of monolayer $h$-BN on several transition metal surfaces, see Fig. 7.1. A $1 \times 1 h$-BN cell fits rather well on a $1 \times 1$ surface unit cell of $\mathrm{Co}(0001), \mathrm{Ni}(111)$, and $\mathrm{Cu}(111)$ and a $2 \times 2 h$-BN cell fits on a $\sqrt{3} \times \sqrt{3}$ surface cell of $\mathrm{Al}, \mathrm{Pd}, \mathrm{Ag}, \mathrm{Pt}$, and $\mathrm{Au}(111)$. The lattice mismatches are then smaller than $2.7 \%$, with the exception of $\mathrm{Pt}$ and $\mathrm{Pt}$, where the mismatches are $3.5 \%$ and $4.6 \%$, respectively. We scale the in-plane metal lattice constants in order to fit to the optimized $h$-BN LDA or vdW-DF lattice constants of $a_{\text {hex }}=2.490$ or $2.510 \AA$, both of which are close to the experimental lattice constant of $a_{\text {hex }}^{\exp }=2.504 \AA$.[122] In a previous DFT calculation of $h$-BN adsorbed on transition metals, $1 \times 1$ cells have been used with $h$-BN scaled to the metals lattice constants.[119] This leads to overstretched $h$ $\mathrm{BN}$, which, depending on the size of lattice mismatch, can give a very unrealistic bonding.[123]

The energetically most favorable binding configurations are shown in Fig. 7.1. In the $1 \times 1$ metal surface cells, the most favorable position for the nitrogen and boron 


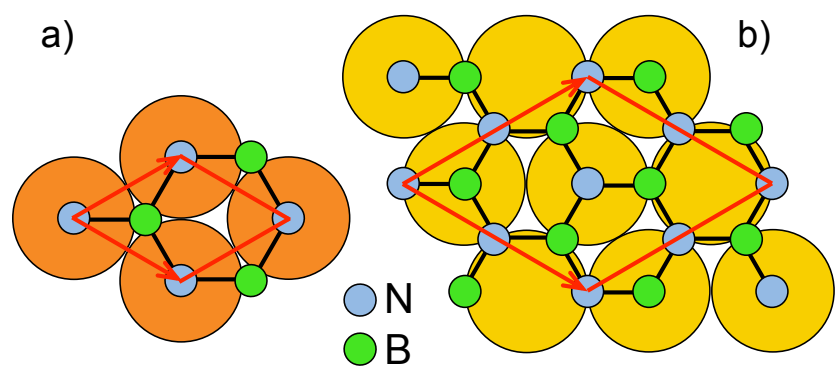

c)
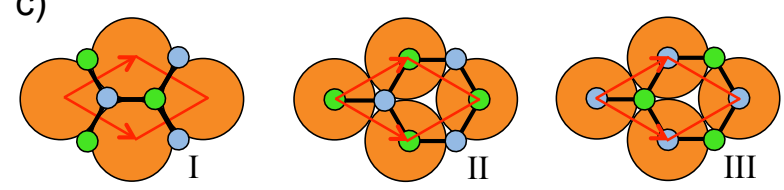

Figure 7.1: The binding configurations of $h$-BN on a) $\mathrm{Cu}(111), \mathrm{Ni}(111), \mathrm{Co}(0001)$ and b) $\mathrm{Pt}, \mathrm{Pd}, \mathrm{Au}, \mathrm{Ag}$ and $\mathrm{Al}(111)$. Figures a) and b) show a $1 \times 1$ and a $2 \times 2 h$-BN unit cell on top of the $1 \times 1$ and $\sqrt{3} \times \sqrt{3}$ surface cells, respectively. c) The three configurations of Table 7.3 ; a) is the same as configuration III.

atoms are top and hollow sites, respectively, see Fig. 7.1a).[99, 124, 119, 105] In the $\sqrt{3} \times \sqrt{3}$ metal surface cells, six of the boron and nitrogen atoms are adsorbed on bridge sites, and two are on top sites, see Fig. 7.1b).

The results of the LDA calculations are shown in Table 7.1. On the basis of the bonding distance $d_{\text {eq }}$ one may expect two different bonding situations. $h$ - $\mathrm{BN}$ is chemisorbed on $\mathrm{Co}(0001), \mathrm{Ni}$ and $\mathrm{Pd}(111)$, as $d_{\mathrm{eq}}<2.5 \AA$, and $h-\mathrm{BN}$ is physisorbed on $\mathrm{Cu}, \mathrm{Pt}, \mathrm{Ag}, \mathrm{Au}$ and $\mathrm{Al}(111)$, as $d_{\mathrm{eq}}>3.0 \AA$. This difference is also apparent in the bonding energies $E_{\mathrm{b}}$ (defined as the difference between the total energies of the combined system minus those of the separate systems). The physisorbed $h$-BN layer has a binding energy in the range of $-50--100 \mathrm{meV} / \mathrm{BN}$, while the binding energy of chemisorbed $h$-BN is in the range of $-150--600 \mathrm{meV}$. The $h$-BN monolayer becomes slightly buckled in the chemisorbed case, with a maximum out of plane distance between boron an nitrogen on $\mathrm{Co}(0001)$ of $0.13 \AA$. There is no significant buckling for the cases where the $h$-BN monolayer is physisorbed.

The adsorption of $h$-BN on metal substrates is quite similar to that of graphene. Graphene is also chemisorbed on $\mathrm{Co}, \mathrm{Ni}$ and $\mathrm{Pd}$, and physisorbed on $\mathrm{Cu}, \mathrm{Pt}, \mathrm{Ag}$, $\mathrm{Au}$ and Al.[73] The bonding distances $d_{\mathrm{eq}}$ of $h$-BN and graphene on these metal surfaces are wthin $10 \%$ of one another, and their order is the same, i.e., $d_{\text {eq }}$ is smallest for $\mathrm{Co}$ and largest for Al. So despite the large difference in electronic structure between graphene and $h$-BN (conductor vs insulator), their bonding to metals is very similar.

Table 7.2 shows the results obtained with the optB88-vdW-DF functional. Comparing the bonding distances to the LDA distances of Table 7.1 we see that the classification into chemisorbed and physisorbed $h$-BN layers is the same as in the LDA. However, the equilibrium bonding distances obtained with optB88-vdW-DF 
Table 7.1: Calculated (LDA) and experimental potential steps $\Delta_{\mathrm{M} \mid \mathrm{BN}}$ at the metal $\mid h$ BN interface; (average) equiilibrium distance $d_{e q}$ between the metal surface and the $h$-BN plane; binding energies $\mathrm{E}_{\mathrm{b}} / \mathrm{BN}$; calculated and experimental work functions $W_{\mathrm{M} \mid \mathrm{BN}}$ of metal $\mid h-\mathrm{BN}$, and $W_{\mathrm{M}}$ of the clean metal surface; the Schottky barrier $\Phi_{p}$ for holes calculated from a $h$-BN monolayer, see Eq. (7.6) with $E_{v}=6.09 \mathrm{eV}$; the Schottky barrier $\Phi_{n}$ for electrons, see Eq. (7.7), where we have used the experimental band gap $E_{g}=5.97 \mathrm{eV}$.[60]

\begin{tabular}{ccccccccccc}
\hline $\mathrm{M}$ & $\Delta_{\mathrm{M} \mid \mathrm{BN}}$ & $\Delta_{\mathrm{M} \mid \mathrm{BN}}^{\exp }$ & $d_{\mathrm{eq}}$ & $\mathrm{E}_{\mathrm{b}}$ & $W_{\mathrm{M} \mid \mathrm{BN}}$ & $W_{\mathrm{M} \mid \mathrm{BN}}^{\exp }$ & $W_{\mathrm{M}}$ & $W_{\mathrm{M}}^{\exp }$ & $\Phi_{p}$ & $\Phi_{n}$ \\
\hline $\mathrm{Co}$ & 1.80 & & 1.92 & -0.583 & 3.72 & & 5.52 & $5.55^{\mathrm{d}}$ & 2.37 & 3.60 \\
$\mathrm{Ni}$ & 1.73 & $1.5-1.8^{a}$ & 1.96 & -0.430 & 3.79 & $3.6^{\mathrm{h}}$ & 5.52 & $5.35^{\mathrm{e}}$ & 2.30 & 3.67 \\
$\mathrm{Pd}$ & 1.25 & $1.3^{b}$ & 2.47 & -0.163 & 4.28 & $4.0^{\mathrm{h}}$ & 5.53 & $5.6^{\mathrm{e}}$ & 1.81 & 4.16 \\
$\mathrm{Cu}$ & 1.18 & $0.8-1.1^{c}, 0.24^{g}$ & 2.97 & -0.087 & 3.99 & & 5.17 & $4.98^{\mathrm{e}}$ & 2.10 & 3.87 \\
$\mathrm{Pt}$ & 1.04 & $0.9^{b}$ & 3.04 & -0.100 & 4.94 & $4.9^{\mathrm{h}}$ & 5.98 & $6.1^{\mathrm{f}}$ & 1.15 & 4.82 \\
$\mathrm{Ag}$ & 0.83 & & 3.20 & -0.070 & 4.00 & & 4.83 & $4.74^{\mathrm{e}}$ & 2.09 & 3.88 \\
$\mathrm{Au}$ & 0.79 & & 3.24 & -0.075 & 4.76 & & 5.55 & $5.31^{\mathrm{e}}$ & 1.33 & 4.64 \\
$\mathrm{Al}$ & 0.41 & & 3.55 & -0.052 & 3.84 & & 4.25 & $4.24^{\mathrm{e}}$ & 2.25 & 3.72 \\
& $(\mathrm{eV})$ & $(\mathrm{eV})$ & $(\AA)$ & $(\mathrm{eV})$ & $(\mathrm{eV})$ & $(\mathrm{eV})$ & $(\mathrm{eV})$ & $(\mathrm{eV})$ & $(\mathrm{eV})$ & $(\mathrm{eV})$ \\
\hline
\end{tabular}

${ }^{a}$ Refs. [87, 98, 99, 100], ${ }^{b}$ Ref. [98], ${ }^{c}$ Ref. [97], ${ }^{d}$ Ref. [125], ${ }^{e}$ Ref. [126], ${ }^{f}$ Ref. [127], ${ }^{g}$ Ref. [87]. ${ }^{h}$ Ref. [128].

Table 7.2: As Table 7.1 but all calculated values obtained with optB88-vdW-DF. The Schottky barrier for holes, $\Phi_{p}$, see Eq. (7.6), is calculated with $E_{v}=6.04 \mathrm{eV}$.

\begin{tabular}{lcccccccccc}
\hline $\mathrm{M}$ & $\Delta_{\mathrm{M} \mid \mathrm{BN}}$ & $\Delta_{\mathrm{M} \mid \mathrm{BN}}^{\exp }$ & $d_{\mathrm{eq}}$ & $\mathrm{E}_{\mathrm{b}}$ & $W_{\mathrm{M} \mid \mathrm{BN}}$ & $W_{\mathrm{M} \mid \mathrm{BN}}^{\exp }$ & $W_{\mathrm{M}}$ & $W_{\mathrm{M}}^{\exp }$ & $\Phi_{p}$ & $\Phi_{n}$ \\
\hline $\mathrm{Co}$ & 1.87 & & 2.02 & -0.348 & 3.55 & & 5.42 & $5.55^{\mathrm{d}}$ & 2.49 & 3.48 \\
$\mathrm{Ni}$ & 1.75 & $1.5-1.8^{a}$ & 2.12 & -0.195 & 3.65 & $3.6^{\mathrm{h}}$ & 5.40 & $5.35^{\mathrm{e}}$ & 2.39 & 3.58 \\
$\mathrm{Pd}$ & 0.87 & $1.3^{b}$ & 3.01 & -0.170 & 4.55 & $4.0^{\mathrm{h}}$ & 5.48 & $5.6^{\mathrm{e}}$ & 1.43 & 4.54 \\
$\mathrm{Cu}$ & 0.82 & $0.8-1.1^{c}, 0.24^{g}$ & 3.27 & -0.140 & 4.28 & & 5.10 & $4.98^{\mathrm{e}}$ & 1.76 & 4.21 \\
$\mathrm{Pt}$ & 0.83 & $0.9^{b}$ & 3.27 & -0.160 & 5.13 & $4.9^{\mathrm{h}}$ & 5.96 & $6.1^{\mathrm{f}}$ & 0.91 & 5.06 \\
$\mathrm{Ag}$ & 0.71 & & 3.37 & -0.131 & 4.11 & & 4.82 & $4.74^{\mathrm{e}}$ & 1.93 & 4.04 \\
$\mathrm{Au}$ & 0.66 & & 3.37 & -0.144 & 4.92 & & 5.58 & $5.31^{\mathrm{e}}$ & 1.12 & 4.85 \\
$\mathrm{Al}$ & 0.38 & & 3.67 & -0.116 & 3.82 & & 4.20 & $4.24^{\mathrm{e}}$ & 2.22 & 3.75 \\
& $(\mathrm{eV})$ & $(\mathrm{eV})$ & $(\AA)$ & $(\mathrm{eV})$ & $(\mathrm{eV})$ & $(\mathrm{eV})$ & $(\mathrm{eV})$ & $(\mathrm{eV})$ & $(\mathrm{eV})$ & $(\mathrm{eV})$ \\
\hline
\end{tabular}

are somewhat larger. For most metals $d_{\text {eq }}$ is larger by a moderate $0.1-0.2 \AA$, but for $\mathrm{Cu}$ it increases by $0.3 \AA$, as compared to LDA, and for Pd it even increases by 0.5 $\AA$. The bonding to $\mathrm{Pd}$ is somewhat special, as is also demonstrated by the binding energies.

The binding energy of $h$-BN on Co and Ni obtained with optB88-vdW-DF is 0.24 $\mathrm{eV} / \mathrm{BN}$ lower than that obtained with LDA. This is consistent with the overbinding one expects LDA to give for the chemisorbed cases. In contrast, the binding energy of $h$-BN on $\mathrm{Cu}, \mathrm{Pt}, \mathrm{Ag}$, $\mathrm{Au}$, and $\mathrm{Al}$ obtained with optB88-vdW-DF is $0.06-0.07 \mathrm{eV} / \mathrm{BN}$ higher than that obtained with LDA. This is consistent with a more proper descrip- 


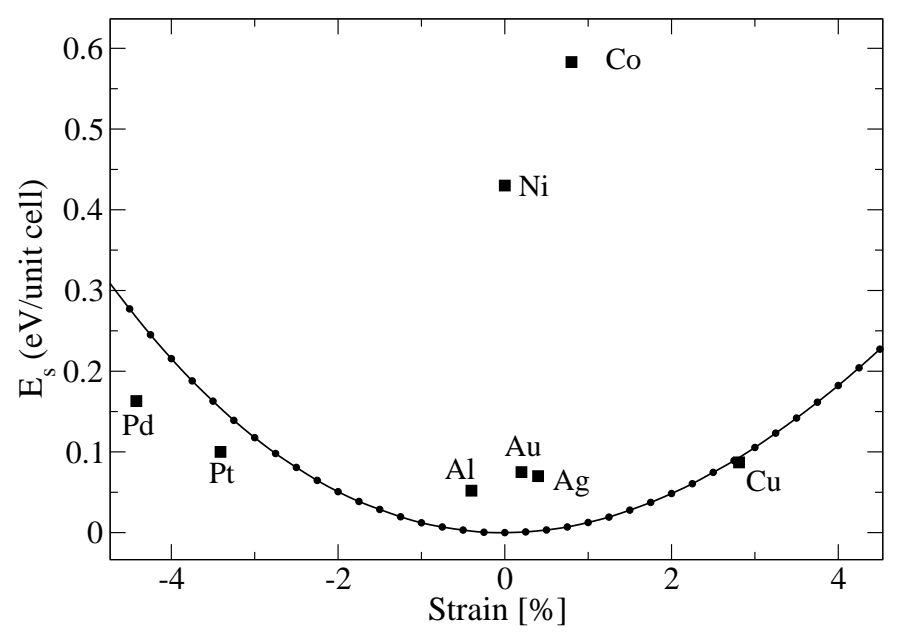

Figure 7.2: Strain energy $E_{s}(\mathrm{eV})$ vesrsus strain (\%) of $h$-BN. The squares give the binding energies $\left|E_{\mathrm{b}}\right|$ of $h$-BN on different metals, see Table 7.1, versus the $h$-BNmetal lattice mismatch.

tion of van der Waals interactions in the vdW-DF, which is important for the physisorbed cases. The binding energies of $h$-BN on Pd obtained with optB88-vdW-DF and LDA are practically the same, which is consistent with the bonding being on the border line between chemisorption and physisorption. Judging from the bonding distances, LDA puts Pd on the chemisorption side of that border, and optB88-vdWDF puts Pd more on the physisorption side.

First-principles calculations are practical only for commensurate structures with reasonably sized unit cells. However, the interaction between the substrate and the adsobant may be too weak to force a commensurate structure in case there is a lattice mismatch between the two. In the present case a question therefore is whether $h$ $\mathrm{BN}$ forms a commensurate structure when deposited on the various metal surfaces. There is a competition between the strain energy of $h$ - $\mathrm{BN}$ and the binding energy to the substrate. We plot the strain energy of $h$-BN versus strain in Fig. 7.2. The LDA binding energies of Table 7.1 are plotted versus the lattice mismatch in the same figure (the vdW-DF binding energies lead to the same qualitative conclusions). A simple rule of thumb states that metal $\mid h$-BN forms a commensurate structure if the gain in binding energy outweighs the cost in strain energy.

For $h$-BN on $\mathrm{Co}(0001)$ and $\mathrm{Ni}(111)$ the lattice mismatches are small and the binding energies are much higher than the strain energy. Therefore it is very likely that $h$-BN will form a commensurate structure on $\mathrm{Co}(0001)$ and $\mathrm{Ni}(111)$ with a unit cell as in Fig. 7.1(a). The uniqueness of this structure would then promote the growth of large areas of defect-free $h$-BN.

If $h$ - $\mathrm{BN}$ is grown on the (111) surfaces of $\mathrm{Al}, \mathrm{Au}$ and $\mathrm{Ag}$ the situation is more complicated. On the one hand, the lattice mismatches are small and the binding energy 
Table 7.3: Calculated binding energies, equilibrium distances and potential steps for $h$-BN on $\mathrm{Cu}(111)$ in the three configurations of Figure $7.1 \mathrm{c})$.

\begin{tabular}{lccc}
\hline Configuration & $E_{\mathrm{b}}$ & $d_{\mathrm{eq}}$ & $\Delta_{\mathrm{M} \mid \mathrm{BN}}$ \\
\hline I & -0.062 & 3.27 & 0.72 \\
II & -0.064 & 3.25 & 0.73 \\
III & -0.087 & 2.97 & 1.18 \\
& $(\mathrm{eV})$ & $(\AA)$ & $(\mathrm{eV})$ \\
\hline
\end{tabular}

is an order of magnitude larger then the strain energy. Commensurate structures with a unit cell as in Fig. 7.1(b) should therefore be stable. On the other hand, this unit cell allows for several structures that have the same energy. For instance, shifting the $h$-BN overlayer by $\left(\frac{1}{3}, \frac{1}{3}\right)$ or rotating it by $60^{\circ}$ with respect to the center of the cell, gives such structures. This means that during growth different domains of $h$-BN can form. A LEED experiment of $h$-BN grown on $\mathrm{Ag}(111)$ indeed shows that $h$-BN domains are formed.[129] Quite likely the same will happen for $h$-BN grown on $\mathrm{Au}$ and $\mathrm{Al}(111)$.

The lattice mismatches for $h-\mathrm{BN}$ on $\mathrm{Cu}, \mathrm{Pd}$ and $\mathrm{Pt}(111)$ are large, and are likely to lead to incommensurate structures. As Figure 7.2 shows, the strain energy needed to stretch the $h$-BN overlayer is comparable to the binding energy. Moreover, some of the binding energy is gained even if the atoms of $h$-BN overlayer are not in their most favorable adsorption positions. The LDA binding energies of three configurations of h-BN on $\mathrm{Cu}(111)$, see Fig. 7.1(c), are given in Table 7.3. These indicate that it is more favorable to adsorb $h$-BN in its equilibrium structure, rather than match it 1:1 with the $\mathrm{Cu}$ surface unit cell. The result is an incommensurate structure.

The difference in bonding distance between the three configurations in Fig. 7.1(c) is $0.30 \AA$. We expect that this is reflected in height variations within a $h$-BN layer on $\mathrm{Cu}(111)$. An incommensurate structure generally leads to the observation of Moiré patterns in the adsorbed layer. Indeed recent STM experiments have shown such patterns in $h$-BN on $\mathrm{Cu}(111)$.[97] The effects of incommensurability on the electronic properties are discussed further in Sec. 7.3.4.

\subsubsection{Metal $\mid h$-BN interface dipole}

It has been shown in experiment that the work function of a metal surface can change substantially upon deposition of a $h$-BN monolayer. The work function modifications reported range from $0.9 \mathrm{eV}$ for $h$-BN on $\mathrm{Pt}(111)$ to $1.8 \mathrm{eV}$ for $h$-BN on $\mathrm{Ni}(111) .[98,99]$ In previous work on Metal|h-BN|graphene stacks we have established that an interface dipole layer is formed at the interface between the metal and the $h$-BN. $[83,105]$ The dipole layer results in a step $\Delta_{\mathrm{M} \mid \mathrm{BN}}$ in the electrostatic potential at the interface, which effectively lowers the work function of the system. This potential step is of key importance for the Schottky barrier, as we will discuss in the next section. 
The potential step corresponding to the dipole layer can be determined by calculating the difference between the (planar averaged) electrostatic potential sufficiently far away from the metal $\mid h$-BN slab and that of the clean relaxed metal surface, assuming a common Fermi level.[75] This is then equivalent to defining the interface potential step as the difference between the work functions of the clean metal surface and the $h$-BN covered surface.

$$
\Delta_{\mathrm{M} \mid \mathrm{BN}}=W_{\mathrm{M}}-W_{\mathrm{M} \mid \mathrm{BN}} .
$$

Alternatively, the interface potential step can be calculated from the electron density difference, defined by subtracting the charge densities of the isolated metal and $h$-BN slabs from that of the combined metal $\mid h$-BN slab

$$
\Delta n(\mathbf{r})=n_{\mathrm{M} \mid \mathrm{BN}}(\mathbf{r})-n_{\mathrm{M}}(\mathbf{r})-n_{\mathrm{BN}}(\mathbf{r}) .
$$

As the system as a whole is neutral and $\Delta n(\mathbf{r}) \rightarrow 0$ for $\mathbf{r}$ sufficiently far from the the metal- $h$-BN interface, solving the Poisson equation with $\Delta n(\mathbf{r})$ as source gives the potential step in terms of the interface dipole[75]

$$
\Delta_{M \mid B N}=\frac{e^{2}}{\epsilon_{0}} \int \Delta \bar{n}(z) z d z
$$

where $z$ is the direction normal to the metal $\mid h$-BN interface, and

$$
\Delta \bar{n}(z)=\frac{1}{A} \iint \Delta n(\mathbf{r}) d x d y,
$$

is the planar averaged electron density difference with $A$ the surface area of the super cell. Numerically, the results of Eqs. (7.1) and (7.3) are within meV's of one another.

The potential steps $\Delta_{\mathrm{M} \mid \mathrm{BN}}$ calculated with the LDA and the vdW-DF functionals, are listed in Tables 7.1 and 7.2 for all the metal $\mid h$-BN structures discussed in this paper. The numbers in Table 7.1 are within $0.2 \mathrm{eV}$ of those in Table 7.2, except for

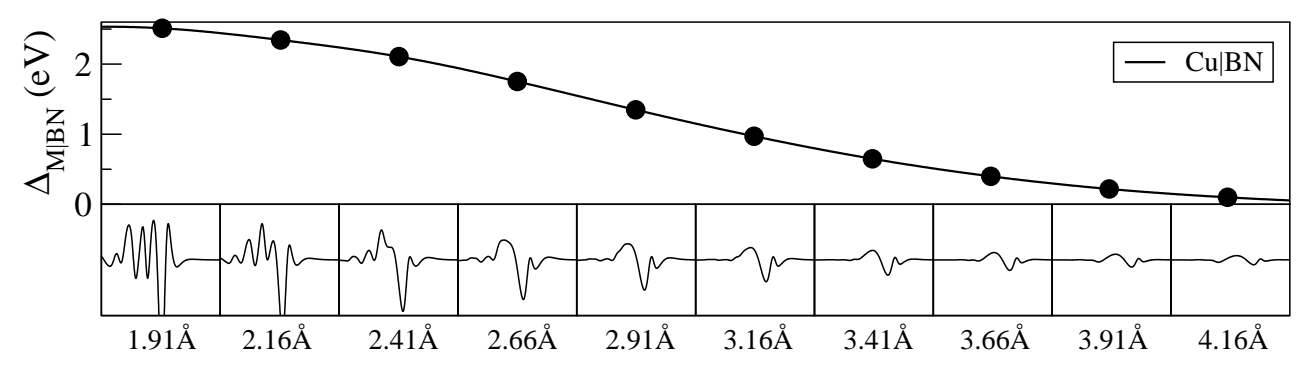

Figure 7.3: (Top) Potential step $\Delta_{M \mid B N}$ at $\mathrm{Cu}(111) \mid h$-BN interface as function of the distance $d$ between the $\mathrm{Cu}(111)$ surface and the $h$-BN plane. (Bottom) Planar averaged electron displacement $\Delta \bar{n}(z)$ at selected distances $d$. 
$\mathrm{Cu}$ and $\mathrm{Pd}$, where the difference is $0.36 \mathrm{eV}$ and $0.38 \mathrm{eV}$, respectively. These differences cannot be ascribed directly to the differences between the functionals. In fact, both functionals give $\Delta_{\mathrm{M} \mid \mathrm{BN}}(d)$ as a function of the metal- $h$-BN distance $d$ that is the same within $0.05 \mathrm{eV}$.[130] However, the functionals give a slightly different equilibrium bonding distance $d_{\text {eq, }}$, see Tables 7.1 and 7.2, and thus a different $\Delta_{\mathrm{M} \mid \mathrm{BN}}\left(d_{\mathrm{eq}}\right)$. These differences are largest for the two metals where the difference between the $d_{\mathrm{eq}}$ obtained with the two functionals is largest, i.e., for $\mathrm{Cu}$ and $\mathrm{Pd}$.

Experimental results for $\Delta_{\mathrm{M} \mid \mathrm{BN}}$, where available, are also given in Tables 7.1 and 7.2. The agreement with the calculated numbers is generally quite good, for both the LDA and the vdW-DF results. For $h-\mathrm{BN}$ on $\mathrm{Cu}(111)$ two quite different results have been obtained for the potential step, i.e., $0.24 \mathrm{eV}$ in Ref. [87] and 0.8-1.1 eV in Ref. [97]. The calculated results suggest that the latter value is more likely to represent well-ordered $h$-BN on clean $\mathrm{Cu}(111)$. A possible origin of the $0.3 \mathrm{eV}$ spread in these measured result is discussed in Sec. 7.3.4.

The potential step for Pd calculated with LDA $(1.25 \mathrm{eV})$ is much closer to the experimental value $(1.3 \mathrm{eV})[98]$, than the vdW-DF value $(0.87 \mathrm{eV})$. Again this does not directly reflect the difference between the two functionals, but rather the difference between the equilibrium bonding distance $d_{\mathrm{eq}}$ these functionals predict. As discussed in the previous section, LDA gives a more chemisorbed $h$-BN layer with a shorter bonding distance ( $d_{\mathrm{eq}} \approx 2.5 \AA$ ) than vdW-DF $\left(d_{\mathrm{eq}} \approx 3.0 \AA\right)$. Comparing the calculated potentials steps to experiment, the shorter bonding distance is more likely.

A typical distance dependence of the potential step $\Delta_{\mathrm{M} \mid \mathrm{BN}}(d)$ is shown in Fig. 7.3 for the $\mathrm{Cu} \mid h-\mathrm{BN}$ interface. Obviously $\Delta_{\mathrm{M} \mid \mathrm{BN}}$ is very sensitive to $d$. The details of this function can be understood in terms of exchange repulsion between the metal surface and the $h$-BN layer at medium distances, which is strongly modified by chemical interactions at shorter distances.[130] The separation in these two regimes can be illustrated by plotting the electron displacement $\Delta \bar{n}(z)$ for different $\mathrm{Cu}(111)-h$-BN distances, as is shown in Fig. 7.3. At distances $d \gtrsim 3.0 \AA \Delta \bar{n}(z)$ shows the pattern of a simple dipole, with accumulation of electrons near the $\mathrm{Cu}(111)$ surface and a concommittant depletion near the $h$-BN plane. Such an accumulation/depletion pattern is also called the pillow effect or the pushback effect, as it seems as if adsorption of the overlayer pushes electrons into the metal substrate. It can be shown that exchange (Pauli) repulsion gives the dominant contribution to the dipole in this distance regime.[130]

At smaller distances, $d<3.0 \AA$, the pattern of $\Delta \bar{n}(z)$ becomes more complicated than that of a simple dipole. The pattern shows oscillations in the metal and this strong perturbation of the electron density in the metal is indicative of the formation of chemical bonds (or anti-bonds). Qualitatively $\Delta \bar{n}(z)$ shows a similar distance dependence for all metal $\mid h-\mathrm{BN}$ interfaces. For metals where the equilibrium distance $d_{\mathrm{eq}}<3.0 \AA$ one observes a pattern with strong oscillations in the metal.[105] These are the metals $(\mathrm{Co}, \mathrm{Ni}, \mathrm{Pd})$ on which $h$ - $\mathrm{BN}$ is chemisorbed according to Table 7.1. In contrast, for metals where the equilibrium distance $d_{\text {eq }} \gtrsim 3.0 \AA$ one observes the 


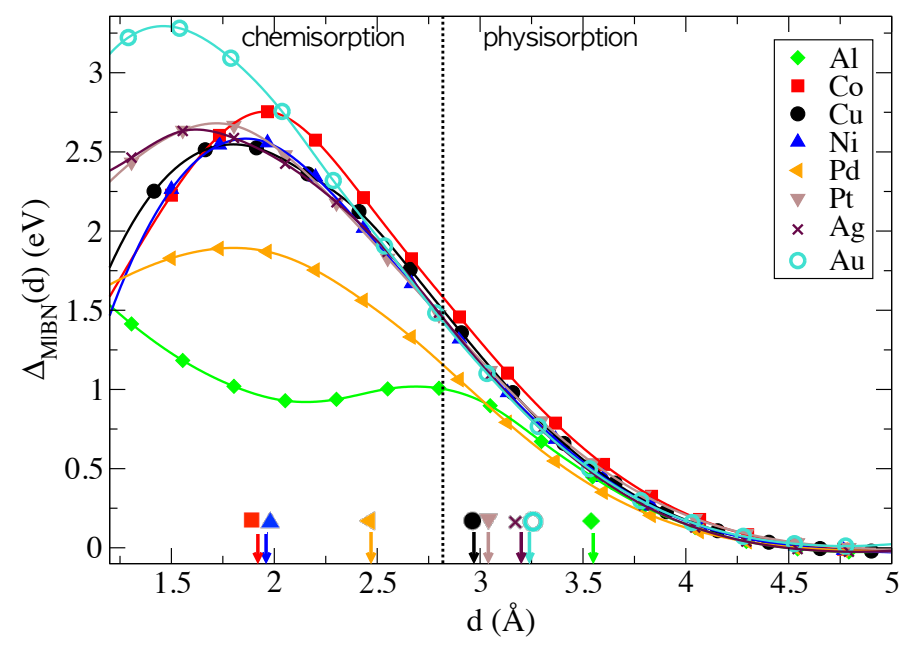

Figure 7.4: Potential steps $\Delta_{M \mid B N}$ at metal $\mid h$-BNinterfaces as function of the distance $d$ between the metal surfaces and the $h$-BN plane. The arrows at the bottom correpond the equilibrium binding distances $d_{\text {eq }}$ of $h$-BN on these metal surfaces, see Table 7.1.

pattern of a simple pushback dipole.[105] These are the metals ( $\mathrm{Al}, \mathrm{Cu}, \mathrm{Ag}, \mathrm{Au}, \mathrm{Pt}$ ) on which $h$-BN is physisorbed according to Table 7.1.

Figure 7.4 shows the potential steps as a function of distance $\Delta_{\mathrm{M} \mid \mathrm{BN}}(d)$ for all the metals considered in this paper, calculated with $h$-BN in a fixed, planar geometry. A distinction is made between chemisorption and physisorption regimes as discussed in the previous paragraph. In the chemisorption regime the curves for different metals can be quite different, which is consistent with the notion that the chemical bonding of $h$-BN to a metal substrate should depend in detail on the type of metal. The potential steps in the chemisorption regime are larger the the ones given in Table 7.1, because we have used a fixed $h$-BN structure for calculating Fig. 7.4. In the chemisorption regime the structure of the $h$-BN layer is perturbed by chemical bonding to the substrate and becomes buckled, which tempers the potential step

Remarkably, in the physisorption regime the $\Delta_{\mathrm{M} \mid \mathrm{BN}}(d)$ curves for the different metals converge. In this regime it is possible to describe all curves with a single function. As in this regime the dominant contribution to the potential step is exchange repulsion between the metal surface and the $h$-BN layer, and exchange repulsion varies roughly exponentially with distance, a reasonable ansatz for a functional form is an exponential function times a polynomial.[130] We find that a second order polynomial gives a sufficiently accurate fit

$$
\Delta_{\mathrm{M} \mid \mathrm{BN}}(d)=e^{-\gamma d}\left(a_{0}+a_{1} d+a_{2} d^{2}\right) .
$$

A least squares fitting procedure of this functional form to the $\Delta_{\mathrm{M} \mid \mathrm{BN}}(d)$ curves for 
the metals on which $h-\mathrm{BN}$ is physisorbed $(\mathrm{Al}, \mathrm{Cu}, \mathrm{Ag}, \mathrm{Au}, \mathrm{Pt})$ then gives $a_{0}=-1865$, $a_{1}=1294, a_{2}=-190.3$ and $\gamma=1.85$.

The fitted function is given in Fig. 7.5 along with the data points, which shows that the fit is remarkably good. An exception seems to be $\mathrm{Al}$ at $d<3.0 \AA$, but this is to be expected as in that regime chemisorption sets in. The deviation of the function from a simple exponential is most prominent at a large distance, i.e., $d>4.0 \AA$. The reason for this is that at large distance the dipole resulting from exchange repulsion goes rapidly to zero. What remains is a small dipole that results from van der Waals interactions between the metal and $h$-BN.[130] The van der Waals dipole does not go exponentially to zero as a function of $d$, but rather as a power law $d^{\alpha}$. In the present case, the numbers become too small to pinpoint the exact value of $\alpha$.

A function of the form given in Eq. (7.5) has also been used by Khomyakov et al. to describe the potential step created in the physisorption of graphene on metal substrates.[72, 73] The bonding of graphene to the metals studied in the present paper is very similar, as is evident by comparing the results for graphene to those given in Table 7.1. In the graphene case the analysis of the potential step is somewhat more complicated, as electrons are transferred between the metal and graphene to equilibrate the Fermi level.[72, 73, 76] As $h$-BN is an insulator such an electron transfer cannot take place across metal $\mid h$-BN interfaces. Modeling the contribution of electron transfer in the metal|graphene case, a contribution to the potential step $\Delta_{c}(d)$ remains that can be fitted with the same functional form as Eq. (7.5). The function $\Delta_{c}(d)$ extracted in Refs. [72,73] is also given Fig. 7.5. For distances in the typical physisorption regime $2.8<d<4.0 \AA$, this function is very close to that obtained

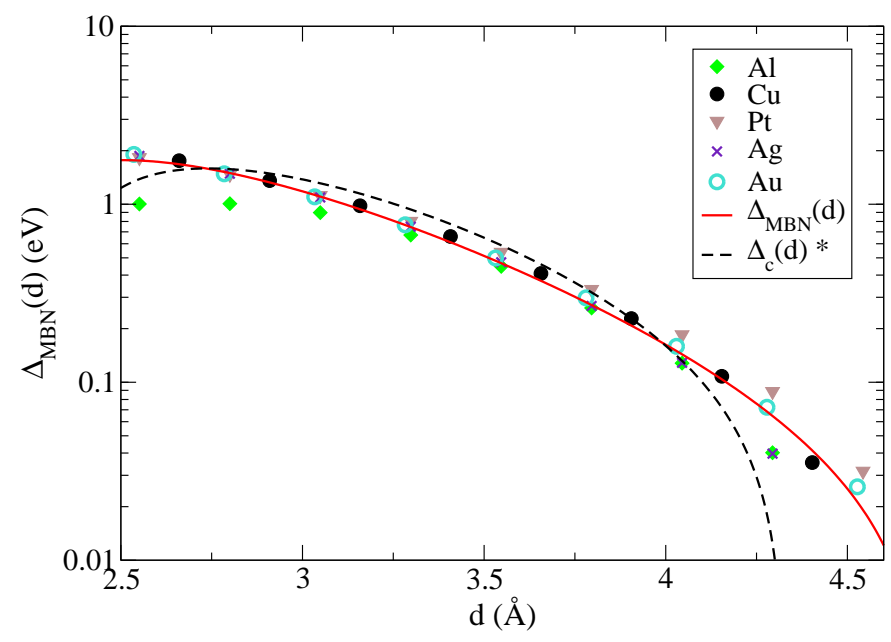

Figure 7.5: Potential steps $\Delta_{M \mid B N}(d)$ at metal $\mid h$-BNinterfaces as in Fig. 7.4 plotted on a logarithmic scale for the metals on which $h$-BN isxorbed. The solid line gives the fiited function of Eq. (7.5); the dashed line gives the function $\Delta_{c}(d)$ used for graphene on these metals in Ref. [73]. 
for metal $\mid h$-BN interfaces, indicating that in their adsorption on metal surfaces, $h$-BN and graphene behave very similar.

\subsubsection{Energy level alignment}

A schematic illustration of the Schottky barriers formed at a metal $\mid h$-BN interface is shown in Fig. 7.6. From this figure it is clear that the $p$-type Scottky barrier is given by

$$
\Phi_{p}=E_{v}-W_{\mathrm{M}}+\Delta_{\mathrm{M} \mid \mathrm{BN}}(d) .
$$

Here $W_{\mathrm{M}}$ is the work function of the clean metal surface, $\Delta_{\mathrm{M} \mid \mathrm{BN}}(d)$ is the interface potential step discussed in the previous section and $E_{v}$ is the position of the top of the valence band of isolated $h$-BN with respect to the vacuum energy (or the ionization potential). We define all these quantities as positive numbers, implying that the $p$ type Schottky barrier is a positive number. With $E_{g}$ the band gap of $h$-BN the $n$-type Schottky barrier is given by

$$
\Phi_{n}=E_{g}-\Phi_{p},
$$

and is also a positive number. The Schottky barriers calculated with Eqs. (7.6) and (7.7) are shown in the last columns of Tables 7.1 and 7.2.

Before discussing these results, we examine in more detail to what extend the electronic structure of $h$-BN is modified upon forming an interface with a metal. Band structures of metal $\mid h-\mathrm{BN}$ interfaces are shown in Fig. 7.7 for some representative metals. If the $h$-BN layer interacts only weakly with the metal substrate, as one expects to be the case for physisorption, then the bands of $h$-BN should still be identifiable. Fig. 7.7 shows that this is indeed the case for $h-\mathrm{BN}$ on $\mathrm{Cu}, \mathrm{Au}$, and $\mathrm{Pt}(111)$,

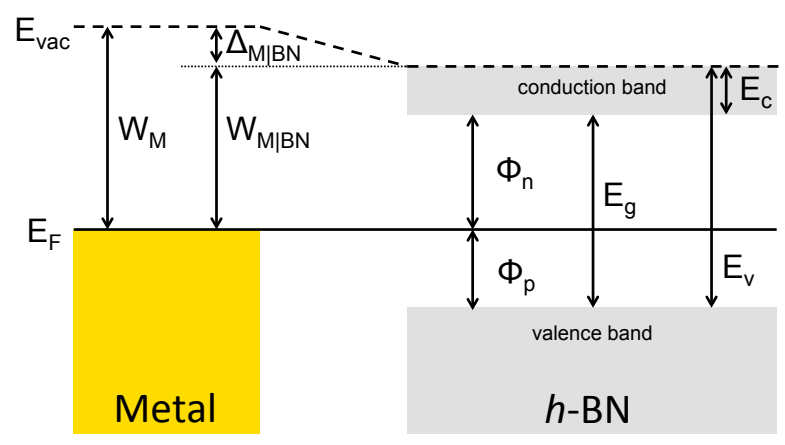

Figure 7.6: Schematic illustration of the energy levels at a metal $\mid h-\mathrm{BN}$ interface; $E_{\mathrm{vac}}$ and $E_{F}$ are the vacuum level and the Fermi level, respectively; $W_{\mathrm{M}}, W_{\mathrm{M} \mid \mathrm{BN}}$, and $\Delta_{\mathrm{M} \mid \mathrm{BN}}$ are the work function of the clean metal surface, that of the metal $\mid h-\mathrm{BN}$ structure, and the interface potential step, respectively; $E_{v}, E_{c}$, and $E_{g}$ are the top of the valence band of $h-\mathrm{BN}$, the bottom of the conduction band, and the band gap, respectively; $\Phi_{p}$ and $\Phi_{n}$ are the Schottky barriers for holes and electrons, respectively. 
for instance. The Schottky barrier $\Phi_{p}=E_{v}-E_{F}$ can be determined from this figure for the different metals, and the results are within $10 \mathrm{meV}$ of those obtained from Eq. (7.6).

If the $h$-BN interacts strongly with the metal substrate, i.e., in case of chemisorption, one does not expect such a simple analysis to hold. Fig. 7.7 also shows the band structure of $\mathrm{Ni}(111) / h-\mathrm{BN}$, where $h-\mathrm{BN}$ is strongly bonded to the metal surface with a short binding distance, see Table 7.1. Somewhat surprisingly, the upper valence band of $h-\mathrm{BN}$ is not strongly perturbed by this interaction. However, it is shifted quite considerably. The top of this band is at $4.4 \mathrm{eV}$ below the Fermi level, whereas the Schottky barrier from Table 7.1 is only $2.3 \mathrm{eV}$. The identification of a single $h$ $\mathrm{BN}$ band in a metal $\mid h$-BN system does not imply that $h-\mathrm{BN}$ is physisorbed, as has been suggested from experiment.[128] In this particular case, for instance, the lower $h$-BN conduction bands are strongly perturbed by the interaction with the $\mathrm{Ni}(111)$ substrate.

If the electronic structure of $h$-BN is strongly perturbed by adsorption the proper value of the Schottky barrier can be recovered by looking at $h$-BN multilayers. As an example, Fig. 7.8 shows the layer projected densities of states (PDOS) of metal|(h$\mathrm{BN})_{n} \mid$ metal structures with seven $(n=7)$ layers of $h$-BN. If $h$-BN is physisorbed on the metal, as is the case for a $\mathrm{Au}(111)$ substrate, the PDOSs of all the $h$-BN layers are very similar, even the PDOSs of the layers that are in direct contact with the metal substrate. This is consistent with the band structure of $h$-BN being unperturbed by adsorption as dicussed above. The Schottky barrier can be determined from the position of the top of the $h$-BN valence band. This position is the same for all $h$ $\mathrm{BN}$ layers, demonstrating that the barrier is located at the $\mathrm{Au} \mid h$-BN interface before the first $h$-BN layer. These conclusions hold for all the metals on which $h$ - $\mathrm{BN}$ is physisorbed (Al, $\mathrm{Cu}, \mathrm{Ag}, \mathrm{Au}, \mathrm{Pt})$.

If $h$ - $\mathrm{BN}$ is chemisorbed (Co, $\mathrm{Ni}, \mathrm{Pd}$ ) the PDOS of the layer that is in direct contact with with the metal, is markedly different from the PDOSs of the other layers. This is illustrated by Fig. 7.8, which shows the PDOSs of a Ni(111) $|h-\mathrm{BN}| \mathrm{Ni}(111)$ structure. The PDOS of the first layer displays the signs of a strong hybridization between the $h$-BN states and the substrate $\mathrm{Ni}(111)$ states. However, already the second layer shows a PDOS that is typical of an unperturded $h$-BN layer. This PDOS allows for an assessment of the Schottky barrier by looking at the top of the $h$-BN valence band. The value obtained is $\Phi_{p}=2.19 \mathrm{eV}$. The difference of $0.11 \mathrm{eV}$ between this and the value of $2.30 \mathrm{eV}$ given in Table 7.1 is caused by a small potential step between the first and the second $h$-BN layer. The first layer is perturbed electronically, as well as structurally (buckled), and its interaction with the unperturbed second layer causes a small potential step. The top of the valence band is then constant throughout the rest of the stack, only the two layers directly at the interface with the metal are affected. This means that in the chemisorbed case the Schottky barrier extends over the $h$-BN monolayer that is adsorbed on the metal. 


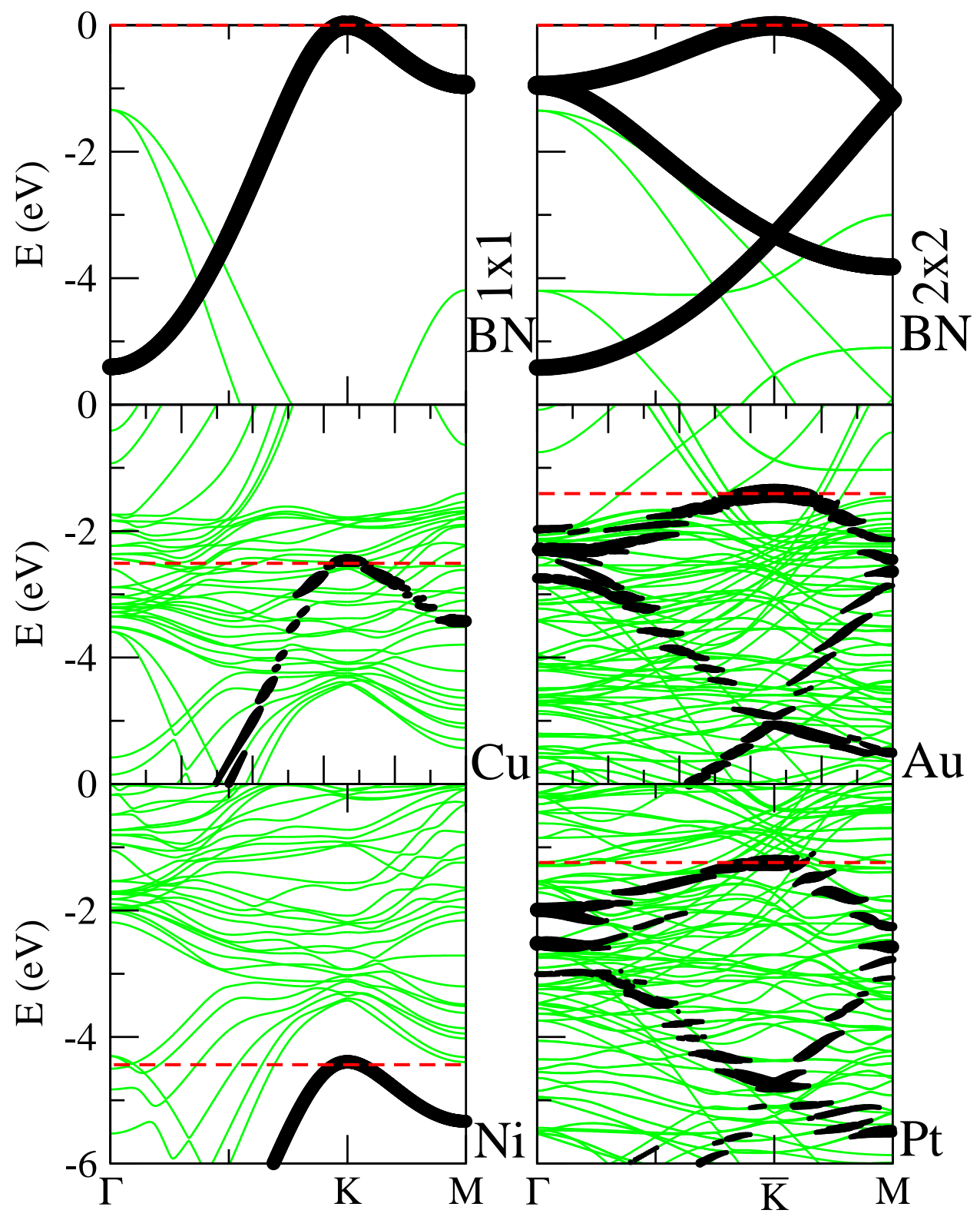

Figure 7.7: Band structures of a $h$-BN monolayer absorbed on the (111)-surfaces of $\mathrm{Cu}, \mathrm{Ni}, \mathrm{Au}$ and Pt. The top two band structures show the bands of isolated $h$-BN as calculated in a $1 \times 1$ and $2 \times 2$ supercell. The black lines of indicate the amount of $h$-BN $\mathrm{p}_{\mathrm{z}}$ character. The red dashed lines indicate the position of the top of the $h$-BN valence band. The zero of energy is put at the Fermi level. 

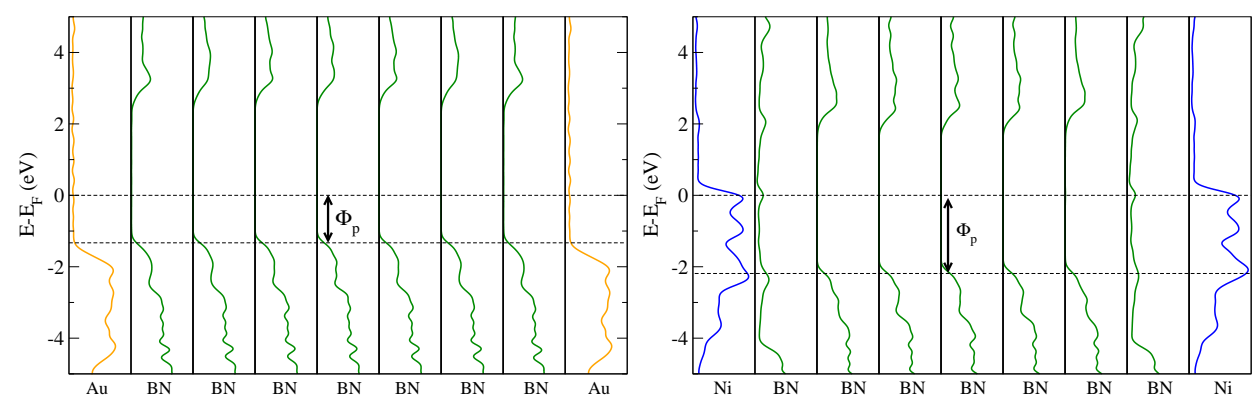

Figure 7.8: Layer projected density of states PDOS of zeven $h$-BN layers sandwiched between (top) $\mathrm{Au}(111)$ and (bottom) $\mathrm{Ni}(111)$ electrodes. The $p$-type Schottky barriers are indicated by $\Phi_{p}$.

\subsubsection{Incommensurate metal $\mid h$-BN systems}

In section 7.3.1 we have argued that $h$-BN on $\mathrm{Cu}, \mathrm{Pt}$ and $\mathrm{Pd}(111)$ forms an incommensurate structure. This means that a superstructure is created with $m \times m h$-BN unit cells on $n \times n$ metal surface unit cells, where the numbers $n, m$ depend on the lattice mismatch and the angle between the $h$-BN and the metal lattices. Recent STM experiments have shown that $h$-BN grown on $\mathrm{Cu}(111)$ shows moiré patterns with periods as large as $14 \mathrm{~nm}$.[97] These experiments also show a spread of $0.3 \mathrm{eV}$ in the local work function depending on the position on the surface, i.e whether the STM tip is on a top or a valley position in the moiré pattern.

Incommensurate structures with large supercells are not directly accessible by first-principles calculations. Therefore we use a simple approach to estimate the spread in the local work function that occurs in an incommensurate structure. We
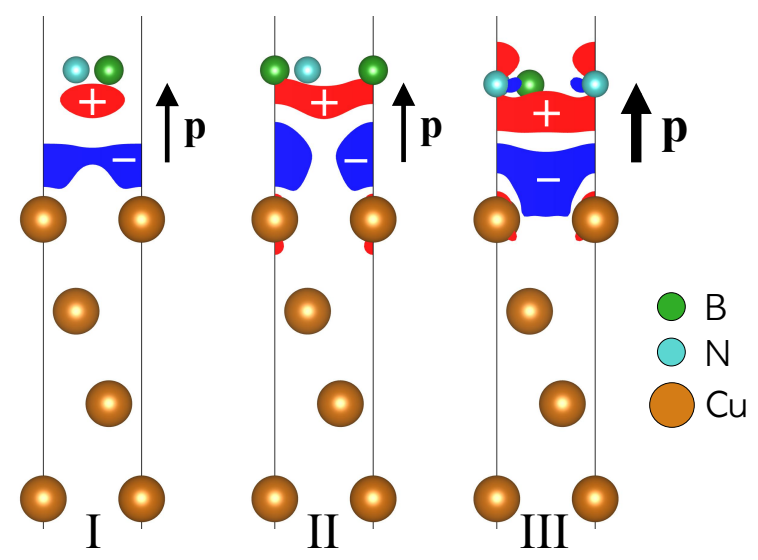

Figure 7.9: Interface dipole for the three different bonding configurations of commensurate $h$-BN on $\mathrm{Cu}(111)$, see Fig. 7.1(c). 


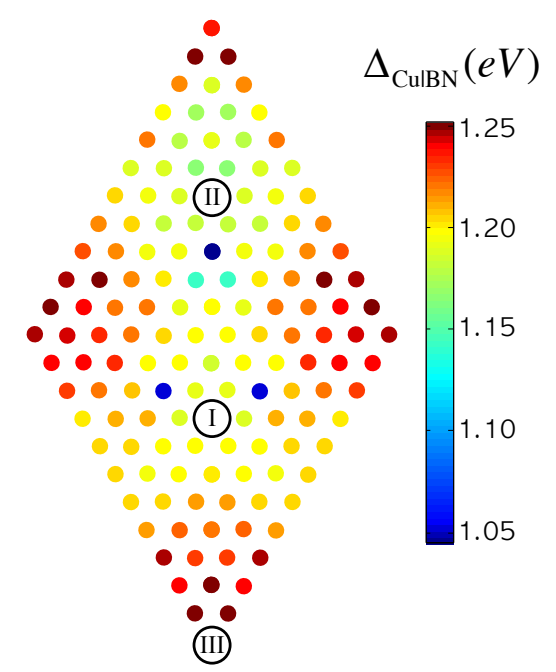

Figure 7.10: The potential step $\Delta_{\mathrm{Cu} \mid \mathrm{BN}}$ at the $\mathrm{Cu}(111) \mid h-\mathrm{BN}$ interface as a function of the in-plane position of $h-\mathrm{BN}$. The $\mathrm{Cu}(111)-h$-BN distance is fixed at $3.0 \AA$. Each dot represents a configuration in steps of $1 / 12$ st of the surface lattice vectors of commensurate $h$-BN on $\mathrm{Cu}(111)$.

consider a fixed orientation of the $h$-BN layer with respect to the $\mathrm{Cu}(111)$ substrate, and do not examine general angles. We do however vary the position of the $h$-BN lattice. The underlying assumption is that in an incommensurate structure with a sufficiently large period, locally the bonding resembles that of a commensurate structure with a specific relative position of the $h-\mathrm{BN}$ and the $\mathrm{Cu}(111)$ lattices.

The local bonding can correspond to that of the configurations I, II and III of Fig. 7.1(c), for instance. The interface dipole is sensitive to the local bonding. This can be clearly seen in Figure 7.9, which shows the charge density difference $n_{\mathrm{Cu} \mid \mathrm{BN}}$ for these three configurations. The size of the interface dipole, and therefore of the interface potential step $\Delta_{\mathrm{Cu} \mid \mathrm{BN}}$, depends on the configuration, see Table 7.3. The dipole is largest if the $\mathrm{N}$ atoms of $h$-BN are adsorbed on-top of the $\mathrm{Cu}$ atoms, as in configuration III, and smallest if both $\mathrm{B}$ and $\mathrm{N}$ atoms are adsorbed at hollow or bridge positions, as in configuration I. The difference in $\Delta_{\mathrm{Cu} \mid \mathrm{BN}}$ between these two configurations is $0.45 \mathrm{eV}$. This difference is slightly larger than that observed in experiment. In our case we use homogeneous structures that are fully relaxed, whereas in an incommensurate structure the intrinsic strain in a $h$-BN layer will prevent the structure from fully relaxing locally to configurations I or III. The value of $0.45 \mathrm{eV}$ is thus likely to be an upper bound for the variation of $\Delta_{\mathrm{Cu} \mid \mathrm{BN}}$.

The binding distance of $h$-BN to $\mathrm{Cu}(111)$ in configuration I is $0.3 \AA$ larger than in configuration III. Choosing a fixed binding distance of $3.0 \AA$ gives a difference in $\Delta_{\mathrm{Cu} \mid \mathrm{BN}}$ of $0.1 \mathrm{eV}$ between the two configurations. Scanning the $h$-BN layer over the $\mathrm{Cu}(111)$ surface at this fixed binding distance gives a potential landscape for $\Delta_{\mathrm{Cu} \mid \mathrm{BN}}$, 
which is shown in Fig. 7.10. The difference between the extrema of this landscape is $0.2 \mathrm{eV}$. This number serves as a lower bound for the variation of $\Delta_{\mathrm{Cu|BN}}$. The interface potential step in an incommensurate structure with a large period varies with the local interface structure, giving rise to a moiré pattern in $\Delta_{\mathrm{Cu} \mid \mathrm{BN}}$.

\subsection{Summary and conclusions}

We calculate the Schottky barriers formed between $h$-BN and a range of metals from first principles, using DFT with vdW-DF and LDA functionals. The close-packed metal surfaces of $\mathrm{Al}, \mathrm{Ag}, \mathrm{Cu}, \mathrm{Ni}, \mathrm{Co}, \mathrm{Pd}, \mathrm{Au}$, and $\mathrm{Pt}$ have work functions ranging from 4.2 to $6.0 \mathrm{eV}$, and their contacts with $h$-BN have Schottky barriers for holes, $\Phi_{p}$, ranging from 0.9 to $2.5 \mathrm{eV}$. There is however not a simple relation between the size of the work function and that of the Schottky barrier. The potential step, $\Delta_{\mathrm{M} \mid \mathrm{BN}}$, caused by the interaction between $h$-BN and the metal at the interface gives a major contribution to the Schottky barrier, cf. Eq. 7.6.

The metal- $h$-BN interaction can be divided into two classes; $h$-BN is chemisorbed on $\mathrm{Co}(0001), \mathrm{Ni}(111)$ and $\mathrm{Pd}(111)$ surfaces, with equilibrium binding distances $\lesssim 2.5$ $\AA$. On the (111) surfaces of $\mathrm{Al}, \mathrm{Cu}, \mathrm{Ag}, \mathrm{Au}$, and $\mathrm{Pt}, h$ - $\mathrm{BN}$ is physisorbed with equilibrium binding distances $\gtrsim 3.0 \AA$. Chemisorption results in large interface potential steps (here, $\Delta_{\mathrm{M} \mid \mathrm{BN}}$ is $1.3-1.8 \mathrm{eV}$ ) that depend on the details of the chemical interaction. Physisorption gives somewhat smaller interface potentials steps (here, $\Delta_{\mathrm{M} \mid \mathrm{BN}}$ is $0.4-1.2 \mathrm{eV}$ ). In the physisorption regime, the dependence of the potential step on the distance $d$ between the metal surface and the $h$-BN plane can be described reasonably well by a single function, cf. Eq. (7.5), which is independent of the metal.

The range of values of the potential step observed for the different metals then reflects the range of the equilibrium binding distances of $h$-BN on these different metals. The differences between the potential steps calculated with vdW-DF and LDA can also be ascribed to differences in equilibrium binding distances obtained with these two functionals. The interface charge distributions obtained with vdW-DF and LDA are very similar, both in the physisorption, as well as in the chemisorption regimes.

In our calculations we force the metal $\mid h-\mathrm{BN}$ interface into a commensurate structure. Such a structure is likely if the mismatch between the $h$-BN and the metal surface lattices is negligible, or in the metal- $h$-BN interaction is strong. However, the lattice mismatch between $h$ - $\mathrm{BN}$ and $\mathrm{Cu}, \mathrm{Pd}, \mathrm{Pt}(111)$ is $\gtrsim 3 \%$, and the metal- $h$ - $\mathrm{BN}$ binding is not sufficiently strong to overcome the strain energy required to compress or stretch $h$-BN to match the metal lattice. Therefore in these cases the metal $\mid h$-BN interface forms an incommensurate structure. Assuming that the period of the resulting superstructure is sufficiently large one can determine an in-plane dependence of the interface potential step from calculations on commensurate systems. For $h$-BN on copper we find an in-plane potential variation in the range $0.2-0.45 \mathrm{eV}$. This leads to an in-plane variation of the Schottky barrier of the same magnitude.

In the chemisorbed cases the structures are likely to be commensurate. The ad- 
sorbed $h$-BN monolayer is then perturbed electronically as well as structurally, i.e., the layer becomes buckled with an amplitude $\sim 0.1 \AA$. The interaction between this perturbed layer and a second $h$-BN layer leads to an additional potential step, whose size however is limited to $\sim 0.1 \mathrm{eV}$.

The calculated work function of $\mathrm{Pt}$ and the $h$-BN ionization potential are very close $(\sim 6 \mathrm{eV})$, yet the calculated Schottky barrier is $\Phi_{p} \approx 1 \mathrm{eV}$. This is caused by a potential step $\Delta_{\mathrm{M} \mid \mathrm{BN}}$ of that size, which is formed upon adsorption of $h-\mathrm{BN}$ on Pt, which effectively lowers the Pt work function. Such potential steps are formed on $h$-BN adsorption on all metal substrates studied in this paper, which implies that all Schottky barriers $\Phi_{p} \gtrsim 1 \mathrm{eV}$. The Schottky barriers for electrons are even higher, i.e., $\Phi_{n} \gtrsim 3.5 \mathrm{eV}$. This means that it will not be easy to apply $h$-BN as a semiconductor material. It also means, however, that $h$ - $\mathrm{BN}$ is a good insulator for use in graphene electronics. 


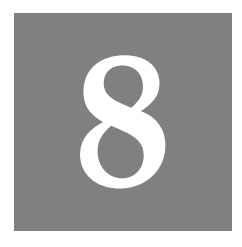

\section{Band gaps in incommensurable Graphene on hexagonal Boron-Nitride}

First-principles calculations are combined with parameterized tight-binding Hamiltonians to study the effects of orientational disorder and incommensurability on the electronic structure of graphene on a hexagonal boron nitride $(h-B N)$ substrate. At the level of density functional theory, the band gap that is opened in commensurable graphene|h-BN by the sublatticesymmetry-breaking interaction with the heteropolar $h-B N$ is quenched to $\leq 5 \mathrm{meV}$ by the $1.8 \%$ lattice mismatch. However, when the Coulomb interaction is taken into account in the GW approximation of many-body perturbation theory, the bandgap is greatly enhanced. We find it to be $\sim 30 \mathrm{meV}$ in the absence of orientational disorder and to be quenched by a small amount of such disorder, consistent with recent experimental results.

\subsection{Introduction}

The development of procedures for preparing single layers of graphene on hexagonal boron nitride substrates [61] has led to an explosion in the number of studies of this system. $h$-BN is a very suitable insulating substrate for making graphenebased devices [80] because it has dielectric characteristics similar to those of $\mathrm{SiO}_{2}$ but contains fewer charged impurities and is atomically flat [84, 85]. These properties result in a higher charge carrier mobility for graphene on $h$-BN compared to graphene on $\mathrm{SiO}_{2}$ [61] and in electron-hole puddles [59] that are larger in size and less deep $[62,63]$.

When graphene is placed on top of $h$-BN it experiences a perturbing potential comprising two components. The $1.8 \%$ lattice mismatch between the two honeycomb lattices and orientational disorder give rise to a slowly varying component that has been observed as moiré patterns in scanning tunneling microscopy images 
$[62,63]$. Because of the chiral nature of the states close to the Dirac point [131], this component does not open a gap [132, 133, 134, 135]; the superlattice Dirac points predicted by these effective Hamiltonian theoretical studies have recently been observed in scanning tunneling spectroscopy experiments [94].

The heteropolar $h$-BN substrate also gives rise to a sublattice symmetry-breaking potential that can open a gap at the Dirac point when graphene and $h$-BN are commensurable [4]. The initial failure $[61,62,63]$ to observe the band gap of order 50 $\mathrm{meV}$ predicted by these first principles calculations was attributed to the lattice mismatch $[62,118]$. On the basis of binding energies calculated from first principles, it was argued that the energy gained by graphene bonding commensurably to $h$-BN was insufficient to offset the energy cost of achieving this by stretching graphene (or compressing $h$-BN). Tight-binding (TB) analyses led Xue [62] and Sachs [118] to argue that the symmetry-breaking interaction between graphene and $h$-BN would average out in the incommensurable case to a much smaller, but not vanishing [136] bandgap.

Recent temperature-dependent transport studies indicating the occurence of a metal to insulator transition at the charge neutrality point at low temperatures suggest that the situation may be more complex [137, 138, 139]. The low temperature insulating state has been interpreted to be induced by disorder (Anderson transition) [137] or to result from substrate-induced valley symmetry breaking [138, 139]. It has been suggested that even small symmetry-breaking-induced band gaps may be greatly enhanced by many body interactions [140].

Because the gap suppression by lattice mismatch depends on details of the approximations made in deriving effective Hamiltonians for graphene [136], we have used first-principles calculations to derive explicit $\pi$-orbital non-orthogonal TB Hamiltonians for graphene on $h$-BN that do not appeal to perturbation theory to fold the graphene-substrate interaction into an effective Hamiltonian. The bandgap induced by a commensurable $h$-BN substrate survives the small lattice mismatch between graphene and $h$-BN but is indeed greatly reduced; it does not survive rotations of the two lattices. Using the LDA eigenvalues, the maximum size of the gap is $5 \mathrm{meV}$. Using many body perturbation theory quasiparticle energies within the GW approximation leads to a large enhancement of the maximum gap to $32 \mathrm{meV}$.

\subsection{Computational details}

We use DFT at the level of the local density approximation (LDA) [16] within the framework of the plane-wave PAW pseudopotential method [14], as implemented in VASP $[44,45,12]$, to determine the electronic structure of a graphene sheet on top of a lattice-matched $h$-BN substrate as a function of the height $z$ and lateral displacement $x, y$ of the two parallel lattices. A plane wave basis with a cutoff energy of 400 $\mathrm{eV}$ was used in combination with a $36 \times 36 \times 1 \mathrm{k}$-point grid and a $15 \AA$ vacuum layer separating the periodically repeated sandwich structures. Many-body effects were studied within the GW approximation [24] starting with LDA Kohn-Sham (KS) or- 
bitals [18] calculated for a cell containing two $h$-BN layers and a graphene layer. We use the frequency dependent GW implementation of VASP [26], with 12 occupied and 52 empty bands and 50 points on the frequency grid. Interactions between periodic images in the $z$ direction lead to a dependence of the GW band gap on the cell size that we remove by linearly extrapolating the calculated gaps as a function of the inverse cell size to infinite separation [141].

\subsection{Density Functional Calculations}

As in our earlier work [4], we distinguish the three high symmetry configurations: (a) with one carbon over B, the other over N; (b) with one carbon over N, the other centered above a $h$-BN hexagon; (c) with one carbon over $\mathrm{B}$, the other centered above a $h$-BN hexagon. The graphene lattice is rotationally aligned with the $h$-BN lattice, the angle $\phi$ between the two lattices is zero. Panels (a)-(c) of Fig. 8.1 show the LDA energy bands for graphene on $h$-BN for the (a), (b) and (c) configurations at their RPA equilibrium separations of $d_{\mathrm{eq}}=3.55,3.50$ and $3.35 \AA$ [118], respectively, with LDA gaps $\Delta \varepsilon=\varepsilon_{3}(\mathrm{~K})-\varepsilon_{2}(\mathrm{~K})$ of $\sim 30-40 \mathrm{meV}$ opened at the $\mathrm{K}$ point by the symmetrybreaking interaction with the substrate [4]. The figure shows that the Dirac point is situated asymmetrically in the $h$-BN bandgap about a third of the way from the top of the valence band. Apart from the formation of the small gap $\Delta$, the dispersion of the $\pi$ bands is largely unchanged by the interaction with the $h$-BN substrate within about $1 \mathrm{eV}$ of the Dirac point. If we expand the scale about the Dirac point (lower panels), we see, however, that the centers of the gaps, $\left[\varepsilon_{2}(\mathrm{~K})+\varepsilon_{3}(\mathrm{~K})\right] / 2$, do not coincide for the different configurations. It turns out that there is an essentially rigid shift of the graphene and $h-\mathrm{BN} \pi$ band structures that is well described by $\Delta V$, a potential step coming from the interaction between graphene and $h$-BN that gives rise to the formation of an interface dipole layer and depends on how the graphene layer and $h$-BN substrate are aligned microscopically. Fig. 8.1(d) shows that the potential steps are not negligible, spanning a range of some $110 \mathrm{meV}$. The electronic displacement $\Delta n(\mathbf{r})$ and dipole formation at the interface can be visualized by examining the electron density of the entire system $n_{\mathrm{Gr|BN}}$ minus the electron densities of the isolated constituent materials, $n_{\mathrm{Gr}}$ and $n_{\mathrm{BN}}[83,105]$

$$
\Delta n(\mathbf{r})=n_{\mathrm{Gr} \mid \mathrm{BN}}(\mathbf{r})-n_{\mathrm{Gr}}(\mathbf{r})-n_{\mathrm{BN}}(\mathbf{r}) .
$$

$\Delta n(\mathbf{r})$ is shown in Fig. 8.1 in a cut parallel to the $y z$ plane containing B, C and N atoms for the (a), (b) and (c) configurations. Regions with electron density above an arbitrary threshold are shown in red and blue and the electron charge density is $\rho=-e n . \Delta n(x, y, z)$ can be related to a potential (energy) step $\Delta V$ by simple electrostatics, $\Delta V=-e^{2} /\left(A \epsilon_{0}\right) \iiint z \Delta n(x, y, z) d x d y d z$, where $A$ is the area of the unit cell in the $x y$ plane.

For graphene displaced with respect to the $h$-BN substrate by $(x, y, z)$ we calculate the full LDA band structure as well as $\Delta V$ on a fine mesh in real space on a 

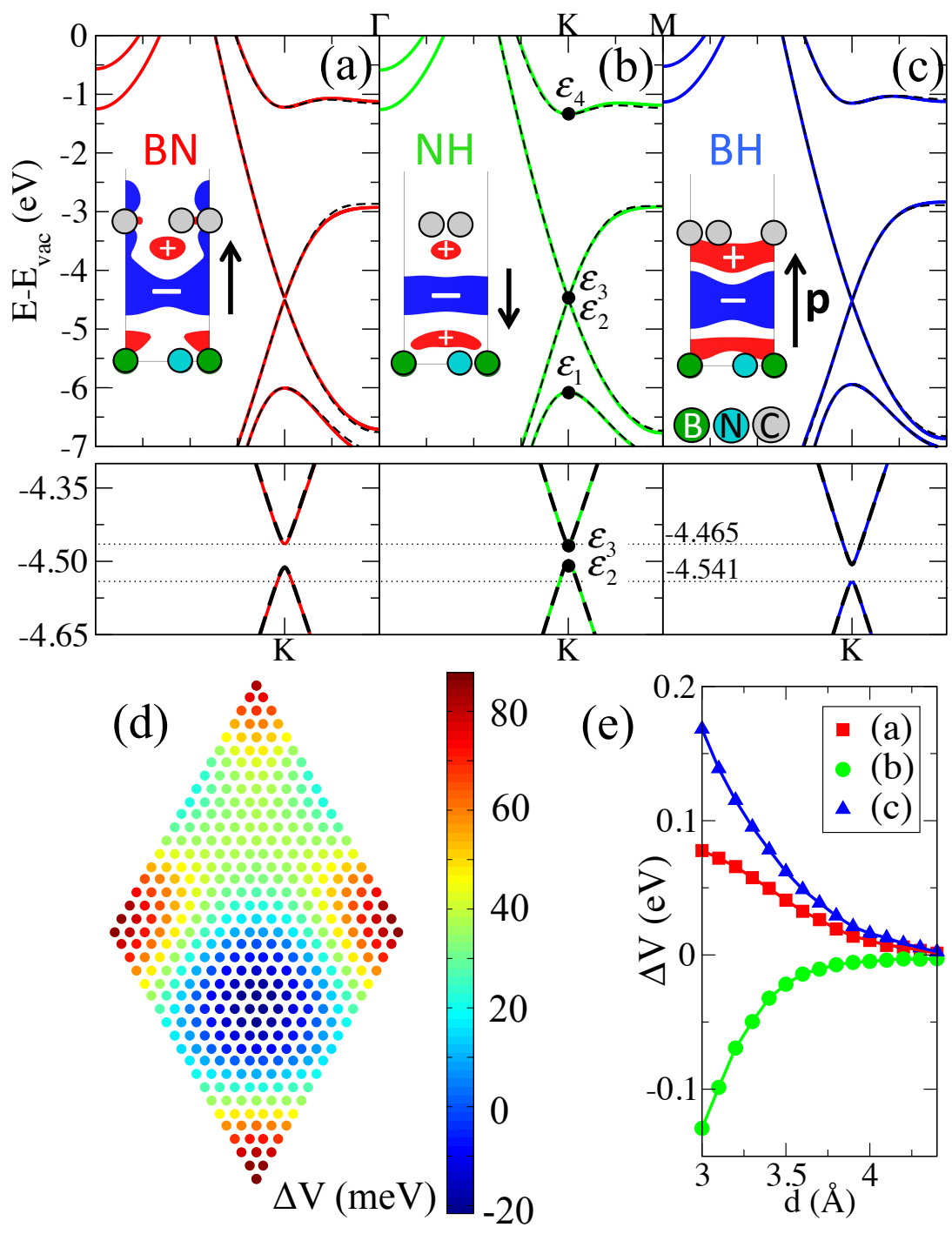

Figure 8.1: LDA electronic structures for graphene on $h$-BN at the equilibrium separations for the (a), (b), and (c) commensurable binding configurations with respect to a common vacuum level. The DFT results are shown by the solid lines and the TB fits are shown by the dashed lines. The bands close to the Dirac point are shown in the panels below on an expanded energy scale. The formation of an interface dipole is illustrated by showing the charge density difference function $\Delta n(x, y, z)$ in a cut parallel to the $y z$ plane containing B, C and $\mathrm{N}$ atoms. Blue and red indicate, respectively, regions of negative and positive charge density (above an arbitrary threshold) that give rise to a dipole moment $\mathrm{p}$. The potential step $\Delta V$ corresponding to the interface dipole $\Delta n(\mathbf{r})$ is shown in (d) as a function of the position $x y$ of the graphene sheet with respect to the $h$-BN substrate at the equilibrium (RPA) separation, see Fig. 8.3a. (e) $z$ dependence of $\Delta V$ for the three high symmetry configurations. 


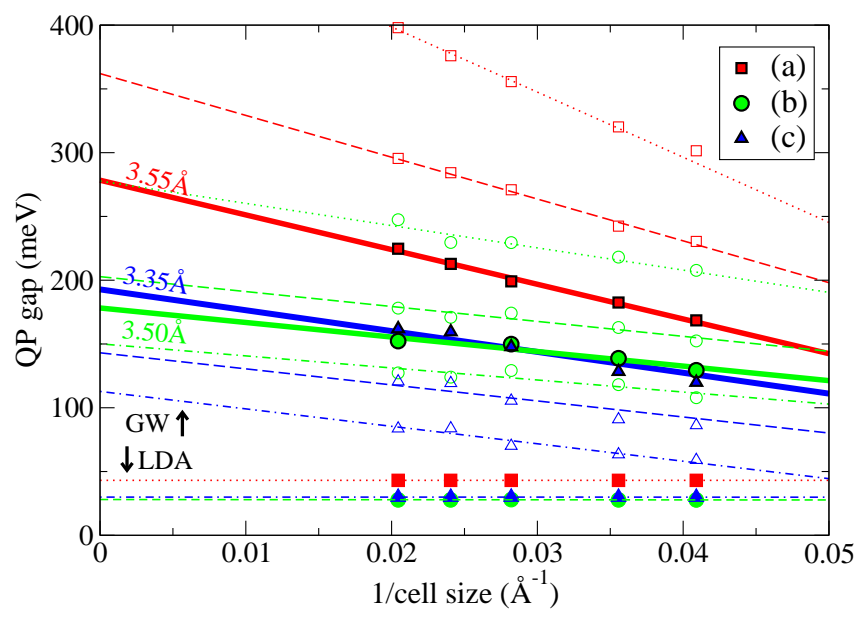

Figure 8.2: Quasiparticle (QP) and, for comparison, LDA gaps opened at the Dirac point as a function of the inverse cell size. The thick solid lines correspond to graphene at the RPA equilibrium distances of configurations (a), (b) and (c). The dotted, dashed and dashed/dotted lines correspond to graphene- $h$-BN separations of $3.35,3.45$ and $3.55 \AA$, respectively.

hexagonal grid in the $x y$ plane, where $z$ is the RPA equilibrium separation in that configuration. The resulting potential step $\Delta V(x, y, z)$ is shown in Fig. 8.1(d). The minimum and maximum values are found for the high symmetry configurations (b) and (c). In Figure 8.1(d), the full $z$ dependence is shown for the (a), (b) and (c) configurations. The interface dipole is seen to vanish for $z>5 \AA$ when there is no overlap between the graphene and $h$-BN wave functions, but does not have any simple analytical form and must be represented numerically.

\subsection{GW correction}

We performed $\mathrm{G}_{0} \mathrm{~W}_{0}$ calculations for the three symmetric configurations in order to find the many body correction to the LDA band gaps in graphene. The quasiparticle gaps are shown in Fig. 8.2 for three separations spanning the range of equilibrium separations calculated within the RPA [118], as a function of the cell size in the direction perpendicular to the graphene sheet.* We are interested in the band gap for an isolated layer of graphene on $h$-BN that we determine by extrapolation to $d \rightarrow \infty$. The gap is seen to increase dramatically, from 43,28 , and $30 \mathrm{meV}$ (horizontal lines) in the LDA to 278, 178 and $193 \mathrm{meV}$, for the (a), (b) and (c) configurations (thick lines), respectively.

*For periodically repeated slabs, the images screen the excitations. For sufficiently large separations, the image potential effect decays as $1 / d$ and we determine the gap for an isolated graphene sheet on $h$-BN by extrapolating to $d=\infty, 1 / d=0[141,142]$. Data points corresponding to the largest cell size were given the greatest weight in the weighted fitting and the average gap, Eq. (8.2), was required to decrease monotonically as a function of the separation. 


\subsection{Band gap in graphene on $h-\mathrm{BN}$}

Before we use the results of the full ab-initio calculations for the commensurable systems to construct a TB Hamiltonian and diagonalize it for the incommensurable case, it is useful to estimate the size of the band gaps we expect to find. We make use of the weakness of the interaction between the graphene and $h$-BN and the large separation in energy between the Dirac point eigenvalues and the $h$-BN valence and conduction band edges to obtain an effective Hamiltonian for the graphene layer by Löwdin downfolding. The substrate can then be replaced by three effective potentials [136]: one that locally shifts the Dirac point; another that opens a local gap; and a third that has the form of a pseudo magnetic field. By applying first order degenerate perturbation theory to the downfolded Hamiltonian, we estimate the gap of the incommensurate system to be

$$
\overline{\Delta \varepsilon}=\frac{\left|\Delta \varepsilon_{(b)}+\Delta \varepsilon_{(c)}-\Delta \varepsilon_{(a)}\right|}{3}
$$

in terms of the gaps for the three commensurable systems. This estimate should be valid in the limit that the mismatch is very small and all possible configurations are sampled equally. The different signs are related to real-space geometrical differences between configurations. Using Eq. (8.2) and assuming that the structure is locally at its equilibrium RPA separation, we expect the band gap of incommensurate graphene on $h$-BN for $\phi=0^{\circ}$ to be $31 \mathrm{meV}$ at the $\mathrm{G}_{0} \mathrm{~W}_{0}$ level compared to $5 \mathrm{meV}$ at the LDA level. Assuming the graphene sheet is flat results in much smaller $G_{0} W_{0}$ gaps of 10,5 and $4 \mathrm{meV}$ for separations of $3.35,3.45,3.55 \AA$, respectively. This suggests that it will be important to take the modulation of the equilibrium separation into account using the "wavy" bonding profile sketched in Fig. 8.3(a). We will find that the gaps estimated using Eq. (8.2) are very close to the results obtained by full numerical diagonalization of the TB Hamiltonian we are now going to construct.

\subsection{Tight Binding Hamiltonian}

The diagonal blocks of the TB Hamiltonian and overlap matrices

$$
H=\left(\begin{array}{ll}
H_{\mathrm{Gr}} & H_{\mathrm{int}} \\
H_{\mathrm{int}}^{\dagger} & H_{\mathrm{BN}}
\end{array}\right) ; O=\left(\begin{array}{cc}
O_{\mathrm{Gr}} & O_{\mathrm{int}} \\
O_{\mathrm{int}}^{\dagger} & O_{\mathrm{BN}}
\end{array}\right)
$$

are first determined separately for monolayers of graphene and $h$-BN. Because isolated monolayers have reflection symmetry, the $p_{z}$ blocks of the corresponding matrices are decoupled from the $\left\{s, p_{x}, p_{y}\right\}$ blocks. By introducing an overlap matrix, $H$ and $O$ can be chosen to have short range and the ab-initio (LDA and GW) bands can be fit essentially exactly. When we consider the interaction between graphene and $h$-BN, we need to include the potential step $\Delta V$ as an additional diagonal term in addition to the off-diagonal blocks $H_{\text {int }}$ and $O_{\text {int }}$. 
To determine Eq. (8.3) for incommensurable lattices, we need to interpolate between the high symmetry (a), (b) and (c) configurations. We make use of its two dimensional lattice periodicity to express $\Delta V(x, y, z)$ as a Fourier series. It turns out that only the shortest reciprocal lattice vectors are needed to describe it very accurately. To define $\Delta V$ when the graphene lattice is rotated through an angle $\phi$ with respect to the $h$-BN substrate, we make use of the fact that $\Delta V(x, y, z)$ has threefold rotation symmetry for a fixed value of $z$ and interpolate for intermediate angles. This approximation was checked for commensurable lattices where explicit DFT calculations can be performed for specific rotation angles for which the unit cell sizes are manageable.

\subsection{TB: Commensurable lattices}

The band structures calculated using the TB Hamiltonian Eq. (8.3) with the parameters fit as described above are compared to the LDA results in Fig. 8.1(a)-(c) (dashed lines). The TB Hamiltonian clearly yields a satisfactory fit for the three configurations shown. It also accurately captures the shift of the Dirac cone as a function of an $x y$ translation of the graphene sheet over the $h$-BN substrate. Figure 8.3(b) shows the position of the Dirac point (the centre of the gap) with respect to the centre of the
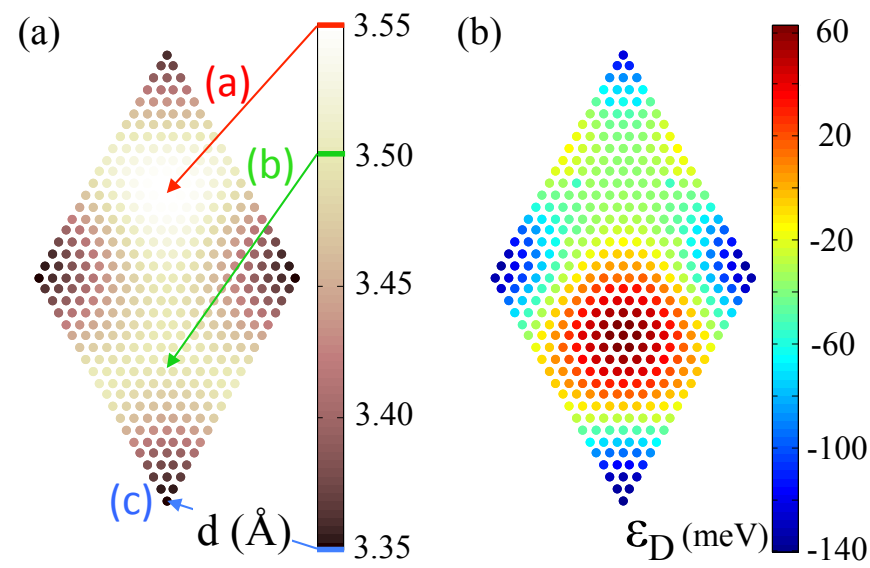

(c)

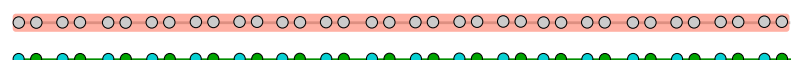

(b)

(d)

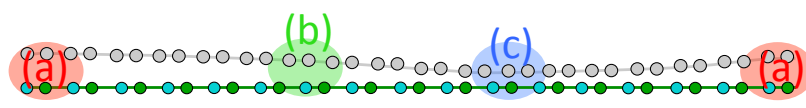

Figure 8.3: (a) The RPA equilibrium separation as a function of the lateral displacement of the two lattices for commensurable graphene on $h$-BN. (b) The position of the Dirac point with respect to the center of the $h$-BN band gap, $\varepsilon_{\mathrm{D}}$. Schematic representations of commensurable (c) and incommensurable (d) configurations of graphene on $h$-BN. Locally the bonding resembles the commensurate configurations. 
$h$-BN band gap

$$
\varepsilon_{\mathrm{D}}(x, y, z)=1 / 2\left(\left(\varepsilon_{3}(\mathrm{~K})+\varepsilon_{2}(\mathrm{~K})\right)-\left(\varepsilon_{4}(\mathrm{~K})+\varepsilon_{1}(\mathrm{~K})\right)\right)
$$

for the height profile shown in Fig. 8.3 (a); it fits the LDA results exactly. Comparison of the LDA energy bands for a structure with graphene rotated through $21.78^{\circ}$ with respect to $h$-BN resulting in a $\sqrt{7} \times \sqrt{7}$ graphene unit cell containing 14 carbon, 7 boron and 7 nitrogen atoms again shows very good agreement. Because the rotated system was not used to parameterize the TB Hamiltonian, the good description of the bands close to the Dirac points is an important measure of its predictive capability. In the case of rotation between the two sheets, the band gap is greatly reduced compared to the unrotated case.

\subsection{TB: Incommensurable lattices}

It has been argued that the bonding between graphene and a $h$-BN substrate is so weak and those honeycomb structures so stiff that the small $1.8 \%$ lattice mismatch will persist in graphene $h$-BN structures [118], see Fig.8.3(d). For perfect alignment, i.e., $\phi=0$, this implies that even if we start at a position where the local bonding corresponds to the lowest energy (BH bonding configuration), the lattice mismatch will result in dephasing of the two lattices. However, the mismatch is sufficiently small that locally the hopping is indistinguishable from a commensurable system displaced by some amount $(x, y)$ so the Hamiltonian for the incommensurable system can be assembled with the parameterized real space Hamiltonian we have just derived. Choosing the vacuum potential as the common potential zero leads to diagonal elements of the TB Hamiltonian that depend on $x, y$ and $z$ and correspond to the local interface dipole. The $h$-BN conduction and valence band edges then undulate in real space, a prediction that could be confirmed by experiment.

Approximating 1.018 with rational approximants such as $m / n$ with e.g. $m=$ $56, n=55$, we construct the TB Hamiltonian for periodic superstructures containing, in this case, $56 \times 56 \times 2$ carbon atoms and $55 \times 55$ each of boron and nitrogen atoms and calculate the low energy eigenvalue spectrum about the Dirac point for the resulting matrix of rank 12322 using sparse matrix and iterative diagonalization techniques.

When $\phi=0$ and assuming that the graphene sheet follows the height profile of Fig. 8.3 (a), we calculate a (GW) band gap of $32 \mathrm{meV}$. This is consistent with reports of the charge neutrality point resistance increasing with decreasing temperature [137, 138] and of gaps of order $30 \mathrm{meV}$ that were extracted from temperature dependent resistivity measurements [139]. The height variation in the structure turns out to be important; using a flat graphene sheet at separations of $3.35 \AA, 3.45 \AA, 3.55 \AA$, results in gaps of $10.3,5.9,5.1 \mathrm{meV}$, respectively, in very good agreement with the gaps estimated using Eq. (8.2).

For rotation angles $\phi>0^{\circ}$, the gap rapidly becomes smaller. We have performed calculations on a $\phi=6^{\circ}, \delta=2.0 \%$ incommensurate structure and have found a zero 
gap. Further calculations on even larger super cells corresponding to smaller angles have to be done to determine the critical angle at which a band gap is induced.

\subsection{Conclusions}

The substrate interaction for rotationally aligned $\left(\phi=0^{\circ}\right)$ graphene on $h$-BN opens up a gap at the Dirac point of graphene that survives the $1.8 \%$ lattice mismatch between graphene and $h$-BN. We calculate a gap of $5 \mathrm{meV}$ at the LDA level, which is enhanced by many body interactions to $32 \mathrm{meV}$ at the GW level, in agreement with recent experiments [139]. The variation of the graphene $h$-BN separation $( \pm 0.1 \AA)$ within the supercell is found to play an important role: without it the gap becomes smaller then $10 \mathrm{meV}$. The gap opening is very sensitive to the rotation angle $\phi$ between the two lattices, a rotation of $6^{\circ}$ being enough to completely quench it. 


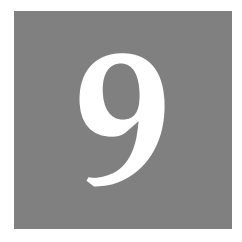

\section{Intrinsic electron-hole puddles in Graphene on hexagonal Boron-Nitride}

When graphene is placed on top of hexagonal boron nitride ( $h-B N)$, the $1.8 \%$ lattice mismatch between the honeycomb lattices of graphene and $h$-BN leads to the formation of superstructures that are observed as Moiré patterns in scanning tunneling microscopy images. We suggest that these patterns have an electrostatic origin. Using first-principles calculations we perceive the formation of a dipole layer at the graphene|h-BN interface. The strength and direction of the dipoles depend sensitively on how the graphene layer is positioned laterally relative to the $h-B N$ layer. The dipole layer gives rise to a step in the electrostatic potential that ranges from +88 to $-22 \mathrm{meV}$ depending on the relative lateral position. The small lattice mismatch between graphene and $h-B N$ leads to a slowly varying relative position, and hence a slowly varying in-plane potential. We suggest that the graphene Dirac cones follow this potential, leading to the formation of regions of electron-and hole-doped graphene: intrinsic electron-hole puddles. In contrast, we predict that graphene on molybdenum disulfide will not result in intrinsic electron-hole puddles.

\subsection{Introduction}

Hexagonal boron nitride $(h$-BN) is a very suitable dielectric substrate for graphene devices. $h$-BN has similar insulating characteristics as $\mathrm{SiO}_{2}$ [84] but has much fewer charged impurities and is atomically flat. This results in a higher mobility of the charge carriers in graphene when placed on $h$-BN instead of $\mathrm{SiO}_{2}$ [61]. The electronhole puddles in graphene on $h$-BN are wide and shallow [59] as compared to those observed in graphene on $\mathrm{SiO}_{2}[62,63]$.

Graphene has a small lattice mismatch with $h$-BN and forms a super-structure 
when placed on $h$-BN, which is observed as a Moiré pattern in scanning tunneling microscopy (STM) images [62, 63]. Since STM does not give a direct measurement of the corrugation, it is possible that the Moiré patterns in graphene on $h$-BN have an electronic instead of a structural origin. If the local density of states at the Fermi level, i.e. the doping level, gradually changes throughout the graphene sheet, the STM tip will follow this profile in order to keep the tunneling current constant.

The $h$-BN honeycomb lattice has approximately a $1.8 \%$ larger lattice constant than graphene. The bonds in the $h$-BN plane have a polar character, because of the large difference in electronegativity of Boron and Nitrogen. It is quite likely that this inplane variation of polarity gives rise to an in-plane varying electrostatic potential. Graphene is weakly bonded to $h$-BN with a binding distance similar to the $h$-BN interlayer binding distance.[4, 118] The competition between strain and binding forces in graphene on $h$-BN has been investigated by means of accurate random phase approximation (RPA) calculations within the framework of the adiabatic connection fluctuation-dissipation theorem (ACFDT).[118] It is found that the energetic cost of straining the graphene sheet to match $h$ - $\mathrm{BN}$ is too large compared to the gain in bonding energy.[118] Therefore the lattice mismatch persists and graphene on $h$-BN forms an incommensurate structure.

Recently it has been reported that the Moiré superlattice gives rise to new Dirac points and to a periodic modulation of the local density of states of graphene on $h$-BN.[94] Charge puddles in graphene in general lead to a reduction of the conductivity. The main conductivity limiting factor in graphene on $\mathrm{SiO}_{2}$ has been attributed to charged impurities in $\mathrm{SiO}_{2}$. [143, 144, 145] Even when a thin film of $h$-BN is inserted between graphene and $\mathrm{SiO}_{2}$ the effect of the charged impurities is still present. The puddle formation in graphene on clean quartz, where the charged impurities are absent, has been interpreted in terms of the corrugation of the graphene sheet.[146]

Neither of these mechanisms, i.e. charged impurities or corrugation of graphene, can explain the charge puddles observed in graphene adsorbed on a thick $h$-BN layer, as the latter is virtually defect-free and the adsorbed graphene is very flat. We use density functional theory (DFT) calculations to study the effects of the incommensurability on the charge redistribution in graphene placed on a $h$-BN substrate. We suggest that the incommensurability introduces a slowly varying interface dipole layer, which leads to the formation of regions of electron- and hole-doped graphene: intrinsic electron-hole puddles. Ultimately, the origin of this effect lies in the polar character of the in-plane bonds in $h$-BN. Such in-plane polar bonds are missing in $\mathrm{MoS}_{2}$. We predict that no intrinsic electron-hole puddles are formed if graphene is placed on $\mathrm{MoS}_{2}$.

\subsection{Computational details}

The DFT equilibrium binding distances and charge densities for the different graphene $\mid h$ BN configurations are calculated using the local density approximation (LDA)[16] and the nonlocal van der Waals density functional (vdW-DF) [23, 112, 113] within the 


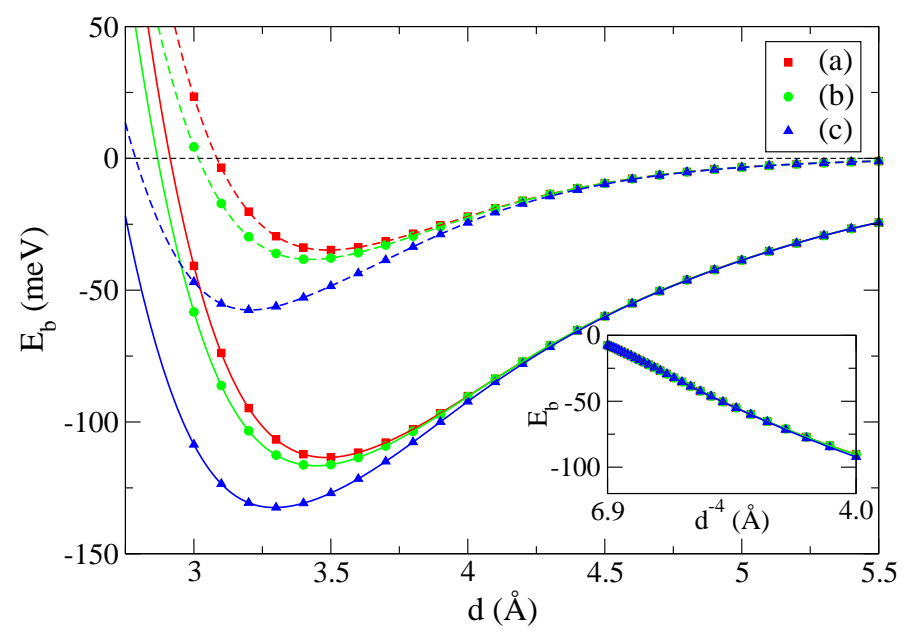

Figure 9.1: Binding energy curves for commensurate graphene on $h$ - $\mathrm{BN}$ in three symmetrical configurations. The dashed lines correspond with the LDA results and the solid lines with the vdW-DF method.

framework of the projector augmented wave (PAW) method[14], as implemented in VASP $[45,147]$. A plane wave basis with a cutoff energy of $400 \mathrm{eV}$ is used. The tetrahedron scheme is applied for calculating total energies with a $36 \times 36 \times 1 \mathrm{k}$-point grid in the graphene Brillouin zone (BZ).[148] The special points $\Gamma, K, K^{\prime}$ and $M$ in the $\mathrm{BZ}$ are explicitly included in the sampling, which is important for an accurate description of the electronic structure around these points. For the supercells one obtains for rotated graphene $\mid h$-BN structures (see below) we use a $6 \times 6 \times 1 \mathrm{k}$-point grid. The same grid is used for the $5 \times 5$ graphene on $4 \times 4 \mathrm{MoS}_{2}$ structure. A dipole correction is applied to avoid spurious interactions between periodic images of the slab.[91] The self-consistency criterion for the total energy is set to $10^{-7} \mathrm{eV}$.

Both $h$-BN and graphite are materials where van der Waals contributions to the binding energy are important. We use the vdW-DF functional[23] and compare the results with those obtained with RPA-ACFDT calculations[118]. The exchangecorrelation functional in vdW-DF is split up as $E_{\mathrm{xc}}=E_{\mathrm{x}}+E_{\mathrm{c}}^{\mathrm{vdW}}+E_{\mathrm{c}}^{\mathrm{loc}}$, where $E_{\mathrm{c}}^{\mathrm{vdW}}$ describes the non-local electron-electron correlations, and $E_{\mathrm{c}}^{\text {loc }}$ describes the local correlations. For these we use the vdW kernel developed by Dion et al.,[23] and the LDA correlation, respectively. For the exchange part $E_{\mathrm{x}}$ we use the optB88 functional.[113] The optB88-vdW-DF functional gives a decent description of the lattice parameters and binding energy of graphite, as well as of the structures and energetics of Li intercalation in graphite.[121] 


\subsection{Graphene $\mid h-\mathrm{BN}$ binding}

Simple commensurate structures are generated by forcing $h$-BN to adapt to the lattice constant of graphene and perfectly align the two lattices. In Figure 9.1 the binding energy of a commensurate and rotationally aligned graphene $h$-BN structure is shown as function of the distance $d$ between the graphene and the $h$-BN planes. We select three different symmetrical configurations; (a) with one carbon over $\mathrm{B}$, the other over $\mathrm{N}$; (b) with one carbon over $\mathrm{N}$, the other centered above a $h$-BN hexagon; (c) with one carbon over $\mathrm{B}$, the other centered above a $h$-BN hexagon. The vdW-DF yields binding energies that are 2-3 times larger than LDA, but similar equilibrium binding distances. The inset shows that the attractive long range tail of the vdWDF curve has a $d^{-4}$ dependence, as expected for the asymptotic behavior for a van der Waals interaction between planes.[22] The LDA curve decays exponentially, as it depends on the wave function overlap between the two sheets.

A comparison to the results from the ACFDT-RPA method is made in Table 9.1. The binding energies obtained with ACFDT-RPA method are in between those obtained with vdW-DF and LDA. However, the energy ordering of the three configurations does not change with the method or functional. The equilibrium separations obtained with the three techniques are similar, with LDA giving somewhat smaller distances than vdW-DF, and ACFDT-RPA somewhat larger distances.

Even though the interaction energy between graphene and $h$-BN is small, a nonnegligible interface dipole layer is formed at the interface.[83] The strength and direction of the dipole layer are determined by the binding configuration as can be seen in Figure 9.2(left) where the dipole layer for the three symmetrical configurations (a), (b) and (c) is shown. The dipoles can be calculated from the charge density difference: $\Delta n(\mathbf{r})=n_{\mathrm{Gr} \mid \mathrm{BN}}(\mathbf{r})-n_{\mathrm{Gr}}(\mathbf{r})-n_{\mathrm{BN}}(\mathbf{r})$. It is convenient to represent the

Table 9.1: Equilibrium binding distances $\left(d_{\mathrm{eq}}\right)$ and energies $\left(E_{\mathrm{b}}\right)$ for commensurate graphene $\mid h$-BN calculated with the LDA, vdW-DF and ACFDT-RPA method. Optimized lattice constants of graphene $a_{\mathrm{Gr}}$ and of $h$-BN $a_{\mathrm{BN}}$.

\begin{tabular}{cccc}
\hline & LDA & vdW-DF & RPA[118] \\
\hline$d_{\text {eq }}(\mathrm{a})(\AA)$ & 3.50 & 3.49 & 3.55 \\
$d_{\text {eq }}(\mathrm{b})(\AA)$ & 3.40 & 3.45 & 3.50 \\
$d_{\text {eq }}(\mathrm{c})(\AA)$ & 3.22 & 3.29 & 3.35 \\
\hline$E_{\mathrm{b}}(\mathrm{a})(\mathrm{meV})$ & 35 & 113 & 62 \\
$E_{\mathrm{b}}(\mathrm{b})(\mathrm{meV})$ & 38 & 117 & 65 \\
$E_{\mathrm{b}}(\mathrm{c})(\mathrm{meV})$ & 58 & 132 & 83 \\
\hline$a_{\mathrm{Gr}}(\AA)$ & 2.445 & 2.465 & - \\
$a_{\mathrm{BN}}(\AA)$ & 2.490 & 2.510 & - \\
\hline
\end{tabular}


effect of the dipole layer by the electrostatic potential step $\Delta V$ that is causes,

$$
\Delta V=\frac{e^{2}}{\epsilon_{0}} \int_{-\infty}^{\infty} z \Delta \bar{n}(z) d z .
$$

where $\Delta \bar{n}(z)=\frac{1}{A} \int_{s} \Delta n(\mathbf{r}) d^{2} r$ is the plane averaged charge density difference.[75]

$\Delta V$ is the change of the potential energy for electrons in the direction perpendicular to the surface. The dependence of $\Delta V$ on the graphene- $h$-BN separation $d$ is plotted in Fig. 9.2(right). The dashed lines correspond to the vdW-DF functional and the solid lines are LDA results. The two curves overlap almost perfectly, proving that the dipole layer is not dependent on the functional used, but is a function of $d$ only. The potential steps corresponding to the RPA equilibrium distances for the (a), (b) and (c) configurations are $+39,-22$ and $+88 \mathrm{meV}$, respectively.

The results shown in Fig. Fig. 9.2(right) indicate that the rather crude LDA functional gives a good description of the charge distribution in this weakly van der Waals bonded system at low computational cost. Therefore in the remainder of this paper we will present LDA based results and use whenever needed the RPA equilibrium distances.

\subsection{Commensurate structures}

Aligning graphene on $h$-BN with a realistic $1.8 \%$ mismatch requires a super cell of $55 \times 55$ graphene and $54 \times 54 h$-BN unit cells. Misaligned graphene $/ h$-BN lattices even lead to larger supercells. Supercells of such size are computationally inaccessible by first-principles calculations at present. In this section we study the potentials steps that occur in (artificial) commensurate graphene $\mid h$-BN interfaces that are obtained by

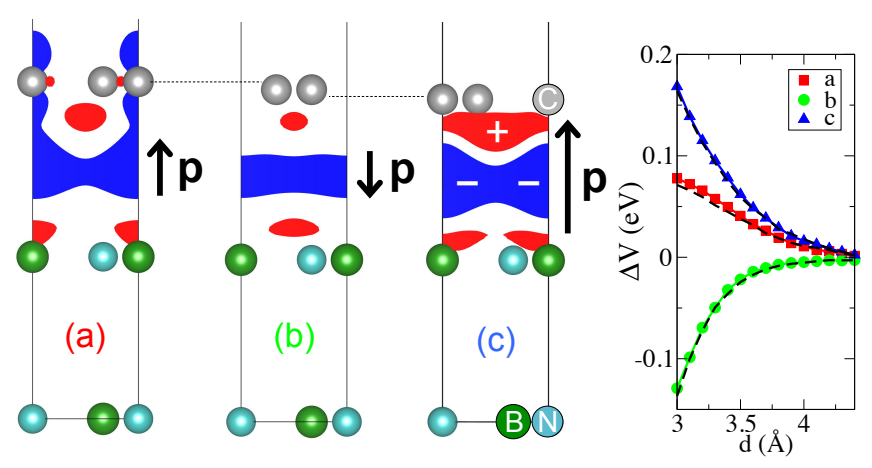

Figure 9.2: Left: side view of the charge density difference $\Delta n$ in three symmetric binding configurations (a), (b). and (c) for graphene on $h$-BN.[149] Blue/red indicates regions of negative/positive charge, which give rise to a dipole moment $\mathbf{p}$. Right: the potential step $\Delta V$ resulting the dipole layer as function of the graphene$h$-BN separation $d$. The solid lines are LDA results, the vdW-DF results are given by the dashed lines. 


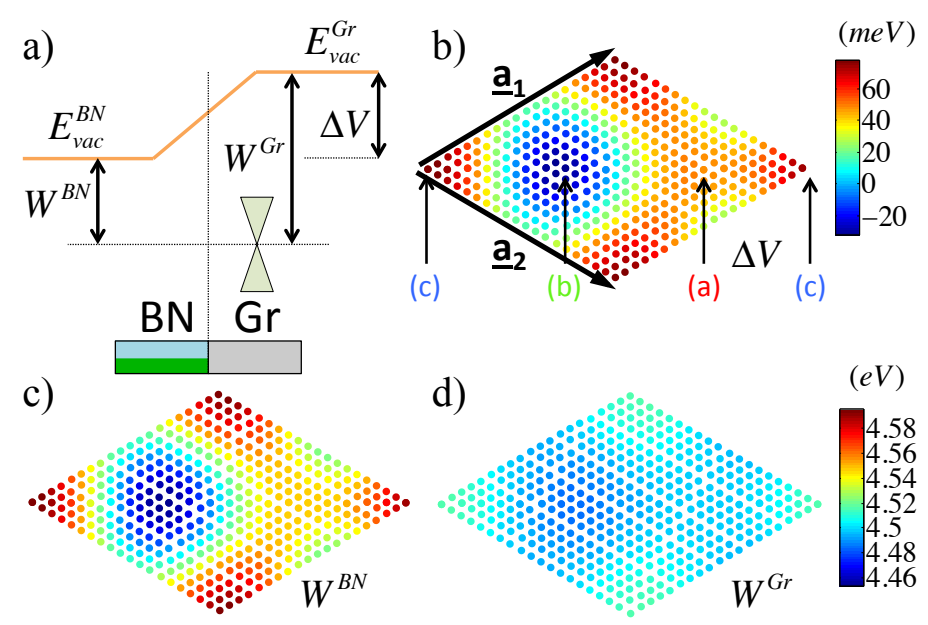

Figure 9.3: a) Energy level diagram of the $h$-BN|graphene interface. The interface potential step $\Delta V$ gives two different work functions $\left(W^{\mathrm{BN}} / W^{\mathrm{Gr}}\right)$ on the two sides of the slab. b) The interface dipole step $\Delta V(x, y)$ as function of $x y$ translation of the graphene sheet over the $h$-BN substrate at a constant graphene- $h$-BN separation of $3.40 \AA$. The work function as function of $x y$ translation of the graphene sheet over the $h$-BN substrate, measured from the $h$-BN side c) and from the graphene side d).

fixing the lattice constant of $h$-BN to that of graphene. In the next section we discuss the consequences of these potential steps for incommensurate structures.

Fixing the lattice constant of $h$-BN to that of graphene, other commensurate structures can be constructed besides the three symmetric configurations presented above. There are essentially three degrees of freedom. The graphene sheet can be translated in the $x y$ plane and it can be rotated w.r.t. the $h$-BN lattice by an angle $\phi$. The size of the graphene $\mid h-\mathrm{BN}$ unit cell does not change for a translation, but it can become very large for an arbitrary angle $\phi$. We will first present results for aligned commensurate systems, $\phi=0^{\circ}$, and study the effects of $x y$ translation of the graphene sheet. Misaligned structures, $\phi \neq 0^{\circ}$, will be discussed later.

We calculate the electronic structure of graphene on top of a $h$-BN monolayer and systematically translate the graphene sheet in plane. The graphene sheet is moved in the $x y$-plane with vectors $x=\frac{n}{20} \mathbf{a}_{\mathbf{1}}, y=\frac{m}{20} \mathbf{a}_{\mathbf{2}}, n, m=0,1, . .19$, creating a (diamond shaped) hexagonal grid of $20 \times 20$ configurations with the (c) configuration as the origin. For each of these configurations we have calculated the interface potential step

$$
\Delta V(x, y)=E_{\mathrm{Vac}}^{\mathrm{Gr}}(x, y)-E_{\mathrm{Vac}}^{\mathrm{BN}}(x, y) .
$$

Results obtained for a constant graphene- $h$-BN separation $d=3.40 \AA$ are shown in Figure 9.3b). The three high symmetry configurations (c), (b) and (a) can be found in this grid at $(0,0),(1 / 3,1 / 3)$ and $(2 / 3,2 / 3)$, respectively. Results obtained from Eqs. (9.1) and (9.2) agree within $10 \mathrm{meV}$. 
A smoothly varying potential landscape $\Delta V(x, y)$ can be seen in the diamond shaped grid of Figure 9.3b) with extrema of +78 and $-32 \mathrm{meV}$ for configurations (b) and (c), respectively. At a larger graphene- $h$-BN distance the shape of $\Delta V(x, y)$ remains the same, but the difference between the potential maximum and minimum decreases. Configurations (b) and (c) give the extrema and that translation of the graphene sheet gives a gradual transition between these. The question now arises what effect $\Delta V$ has on the electronic structure of graphene.

The interaction between the graphene and $h$-BN sheets in a commensurate structure opens up a band gap, splitting the eigenvalues of the tips of the Dirac cone into $\epsilon_{\mathrm{con}}^{\mathrm{Gr}}$ and $\epsilon_{\mathrm{val}}^{\mathrm{Gr}}$. [4] We use the center of the band gap as a measure for the position of the center of the Dirac cone. Assuming the graphene sheet is undoped, this position is equal to the work function $W$ of the slab. As the vacuum level changes by $\Delta V$ along the normal to the slab, the work function for extraction of an electron on the graphene side is different from that on the $h$-BN side

$$
W^{\mathrm{Gr} / \mathrm{BN}}=E_{\mathrm{Vac}}^{\mathrm{Gr} / \mathrm{BN}}-\frac{\epsilon_{\mathrm{con}}^{\mathrm{Gr}}+\epsilon_{\mathrm{val}}^{\mathrm{Gr}}}{2},
$$

where $E_{\mathrm{Vac}}^{\mathrm{Gr}} / E_{\mathrm{Vac}}^{\mathrm{BN}}$ are the vacuum levels on the graphene $/ h$-BN sides of the slab. A schematic picture of these energy levels is shown in Figure 9.3a).

In Figures $9.3 \mathrm{c}$ ) and d) the vacuum level w.r.t. the position of the Dirac cone $\left(W^{\mathrm{Gr} / \mathrm{BN}}\right)$ on both sides of the slab is plotted. Figure 9.3c) shows that the position of the Dirac cone $W^{\mathrm{BN}}(x, y)$ depends sensitively on the relative $x y$ positions of the graphene and the $h$-BN sheets. In fact, the shape and the amplitude of $W^{\mathrm{BN}}(x, y)$ is almost the same as that of $\Delta V(x, y)$, which indicates that the Dirac cone is shifted by $\Delta V$, see Figure 9.3a). This is confirmed by Figure 9.3d), which shows that the position of the Dirac cone $W^{\mathrm{Gr}}$ varies by only $\pm 10 \mathrm{meV}$ around the work function of pristine graphene $(4.48 \mathrm{eV})$. This variation is caused by the interaction between graphene and $h$-BN, which opens a band gap in graphene asymmetrically depending on the relative $x y$ position.

So far we have looked at perfectly aligned graphene and $h$-BN lattices. Rotating the graphene lattice by an angle $\phi$ generally leads to very large supercells, except for special angles. Structures obtained for five such special angles are shown in Figure 9.4. Here, the lattice constant of $h-\mathrm{BN}$ is still forced to be identical to that of graphene. The larger the unit cell, the easier it is to identify local regions where the registry of the graphene and $h$-BN lattices resembles that of the aligned lattices discussed above. Regions resembling the symmetric structures (a) and (b) are indicated in Figure 9.4. We will come back to this point in the next section.

Throughout a unit cell of a rotated structure the carbon atoms have a slightly different environment, resulting in an inhomogeneous dipole layer. Figure 9.5 shows the charge density difference $\Delta n(\mathbf{r})$ of a $\phi=13.17^{\circ}$ rotated graphene $\mid h$-BN commensurate unit cell, obtained with a graphene- $h$-BN separation of $3.22 \AA$. Locally the interface dipole layer resembles the ones shown in Fig. 9.2 and others in between. The planar average of the interface dipole layer over the entire cell then gives a potential 


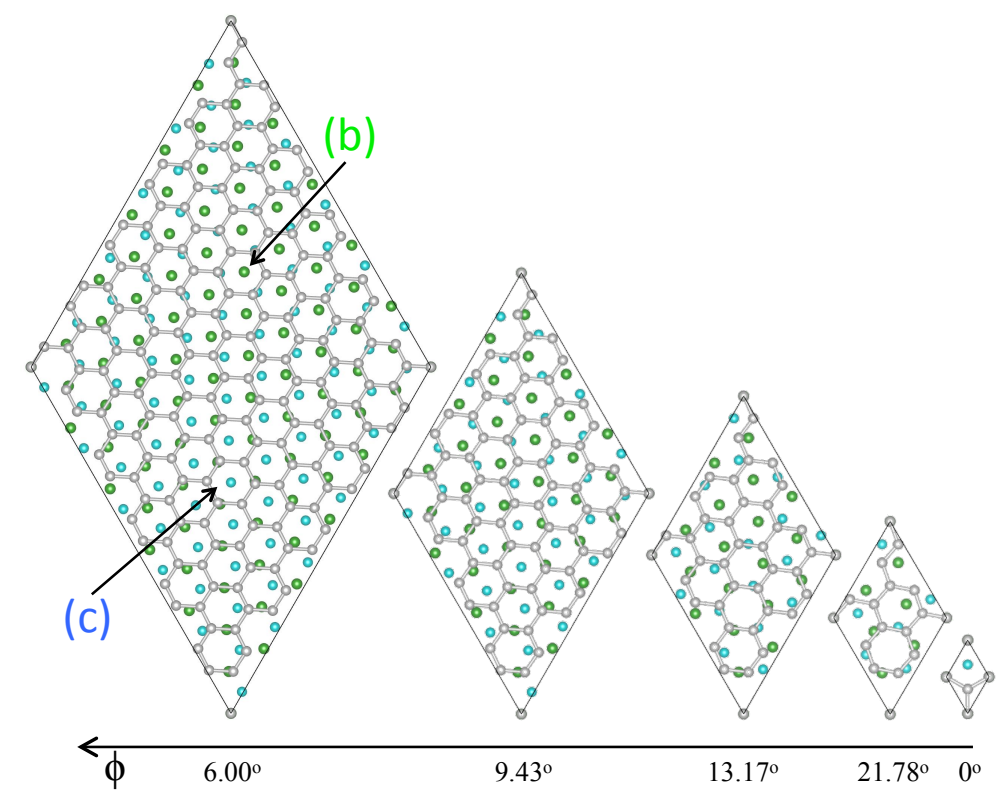

Figure 9.4: Unit cells of commensurate graphene on $h$-BN structures for special rotation angles $\phi$ between the two lattices. If the angle between the two lattice is small, regions that closely resemble the symmetric configurations (b) and (c) can be identified.

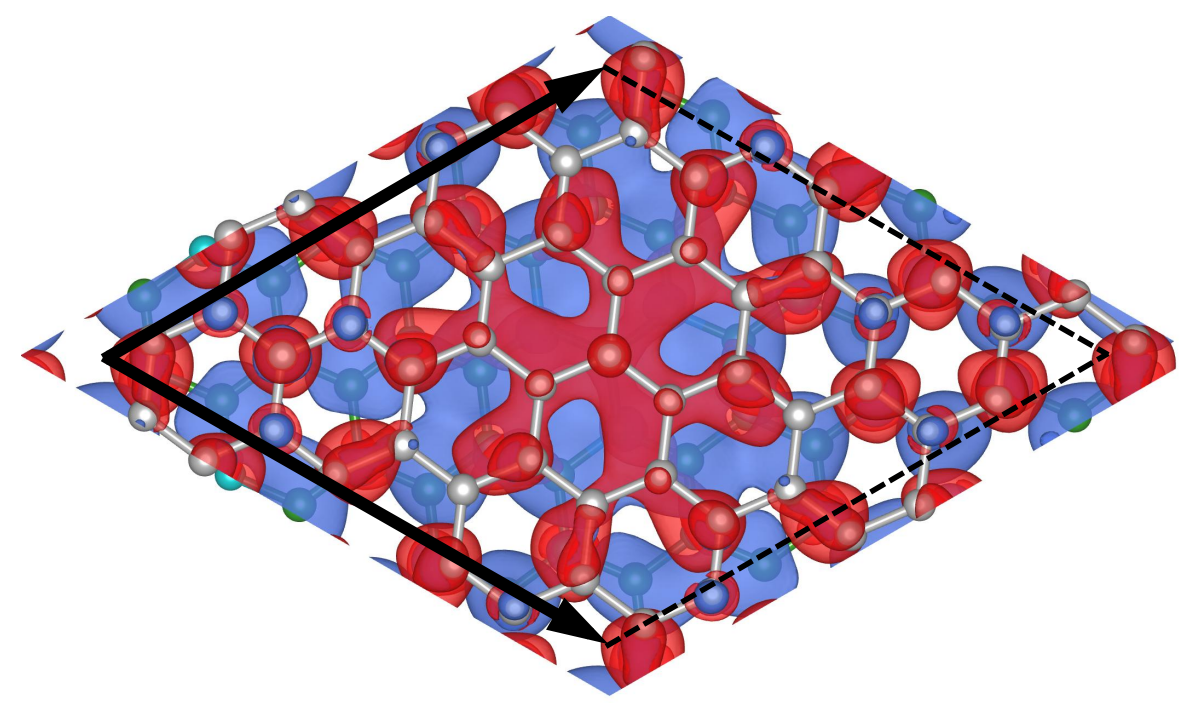

Figure 9.5: Top view of the charge density difference $\Delta n(\mathbf{r})$ of a $\phi=13.17^{\circ}$ rotated graphene $\mid h$-BN commensurate structure. Blue/red indicate regions of negative/positive charge leading to a inhomogeneous dipole layer. 
step $\Delta V$ of approximately $\sim+50 \mathrm{meV}$, for the structures shown in Fig. 9.4, independent of the angle. This is close to the average of $\Delta V(x, y)$ at the same graphene- $h$-BN separation is $+39.3 \mathrm{meV}$. However, this does not mean that the potential is constant in the plane of the unit cell.

We study the in-plane inhomogeneity of the local potential by comparing the $1 \mathrm{~s}$ core levels of the carbon atoms. These levels are good indicators of the variation of the local electrostatic potential because the 1s states are localized at the atomic sites. The 1 s levels of the carbon atoms in freestanding graphene are all at the same energy, but the interaction with the $h$-BN sheet shifts them. In the aligned graphene $\mid h$-BN commensurate structures $\left(\phi=0^{\circ}\right)$ the symmetry between the two carbon sublattices is broken, which results in a different shift for these two sublattices, depending on their $x y$ position with respect to $h$-BN. The difference between the sublattices in the 1s level shifts are 54, 48 and $11 \mathrm{meV}$ for the (a), (b) and (c) structures, respectively, at a graphene $\mid h$-BN separation of $3.22 \AA$. We use these numbers to characterize these structures.

For the rotated commensurate structures we consider the difference $\Delta \epsilon_{i}^{1 \mathrm{~s}}$ between the $1 \mathrm{~s}$ carbon levels and the average $1 \mathrm{~s}$ level,

$$
\Delta \epsilon_{i}^{1 \mathrm{~s}}=\epsilon_{i}^{1 \mathrm{~s}}-\frac{1}{N} \sum_{i=1}^{N} \epsilon_{i}^{1 \mathrm{~s}} .
$$

Figure 9.6(top) shows $\Delta \epsilon^{1 \mathrm{~s}}$ for the $\phi=13.17^{\circ}$ structure. A landscape with a similar symmetry as $\Delta n(\mathbf{r})$ in Fig. 9.5 appears. The maximum difference between the shifts is $\sim 27 \mathrm{meV}$. Apart from the translational symmetry associated with the unit cell there is no simple recognizable pattern.

Such a pattern emerges, however, if the unit cell is sufficiently large. Figure 9.6 (bottom) shows $\Delta \epsilon^{1 \mathrm{~s}}$ for the $\phi=6^{\circ}$ structure, where a pattern can be identified. In a large cell there are regions that are similar to the symmetric configurations in the small cells of the $\phi=0^{\circ}$ structures. In particular, (b) and (c) like regions in the $\phi=6^{\circ}$ structure can be identified in Fig. 9.6(bottom), because adjacent carbon atoms have approximately the same difference in $1 \mathrm{~s} \mathrm{level} \mathrm{as} \mathrm{in} \mathrm{the} \mathrm{(b)} \mathrm{and} \mathrm{(c)} \mathrm{structures.} \mathrm{This} \mathrm{is}$ consistent with the structure shown 9.4, where the $\phi=6^{\circ}$ structure has a boron atom below the center of the graphene hexagon on the (b) site and a nitrogen atom below a graphene hexagon center on the (c) site.

The unit cell increases in size with decreasing misalignment angle $\phi$. The variation in $\Delta \epsilon^{1 \mathrm{~s}}$ in the graphene plane converges to that in the aligned commensurate cell. If locally in the large cells a difference between the $1 \mathrm{~s}$ carbon levels is found that is comparable to the difference in the $1 \mathrm{~s}$ levels of the symmetric configurations, then the potential step $\Delta V$ is also the same at that point. 

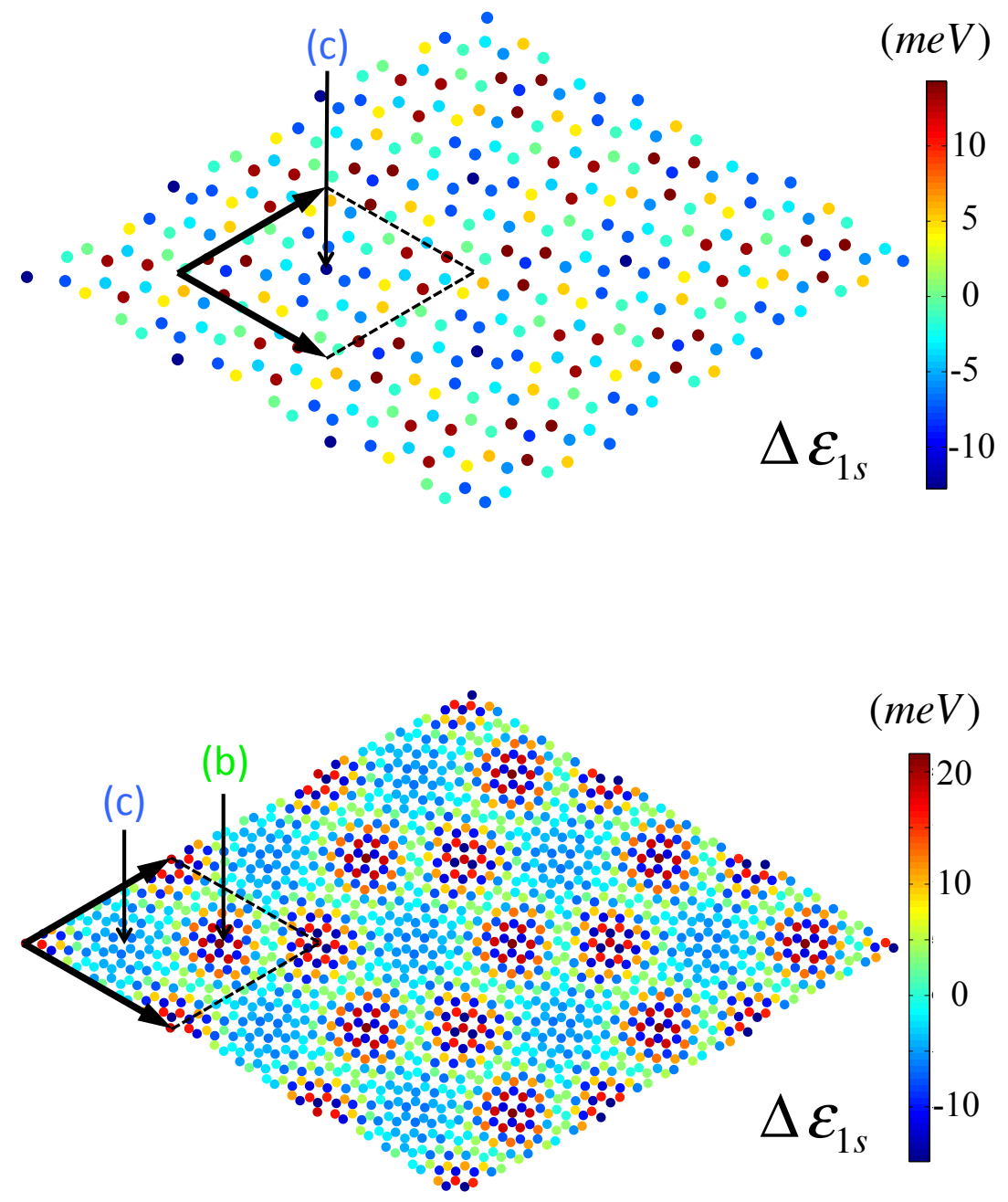

Figure 9.6: 1s carbon core level differences $\left(\Delta \epsilon^{1 \mathrm{~s}}\right)$ in a $\phi=13.17^{\circ} / 6.00^{\circ}$ (top)/(bottom) rotated graphene $\mid h$-BN commensurate super cell with a graphene- $h$ $\mathrm{BN}$ separation of $3.22 \AA$. Regions that closely resemble the symmetric configurations (b) and (c) can be identified in the larger unit cell. 


\subsection{Puddle formation}

At an actual graphene $\mid \mathrm{h}-\mathrm{BN}$ interface one has a $1.8 \%$ mismatch between the graphene and the $h$-BN lattices. Additionally one can have a orientational misalignment between the two lattices. These two effects combined result in Moiré patterns with a period as large as 10-14 $\mathrm{nm},[139]$ which is roughly five times larger than the biggest unit cell considered in this paper, see Figure 9.4. The local in-plane registry of the $h$-BN and graphene lattices slowly varies, and the interface dipole and potential step vary according to the local structure according to Figure 9.3b).

In an actual device graphene is deposited on a (thick) layer of $h$-BN that is placed on top of a metal gate electrode.[94] The potential energy level on the $h$-BN side in Figure 9.3 a) is then fixed by the gate electrode and the variation of the graphene $\mid h$ BN interface potential step $\Delta V(x, y)$ is then felt as a potential landscape in the graphene plane. If the periodicity of this potential is sufficiently large one cal still define locally a Dirac cone. This is shifted according to the local potential, as illustrated by Figure 9.7(top). Establishing a common Fermi level in the system then results in regions in the graphene plane that are $p$-doped, and other regions that are $n$-doped, or in other words, in electron-hole puddles.

These puddles have the periodicity of the interface potential step $\Delta V(x, y)$, and thus of the Moiré pattern formed by the misaligned graphene and $h$-BN lattices. This has been observed in STM experiments where a periodic modulation of the local density of states (LDOS) has been measured with the period of the structural Moiré pattern.[94] If the (local) electronic structure of graphene is described by a Dirac cone, the LDOS at the Fermi level is proportional to the difference between the Fermi level and the conical point of the Dirac cone, i.e. the local doping level, see Figure 9.7(top).

The Fermi level in graphene can be varied by applying a gate voltage.[83, 105] Moving the Fermi level in Figure 9.7(top) changes the local doping level and the LDOS, while maintaining the in-plane periodicity. This has indeed been observed in experiment.[94]

A small band gap in graphene is predicted by first principles calculations in commensurate and aligned graphene $\mid h$-BN structures.[4] A gap has not been observed in most experiments, $[61,62,63]$ which has been attributed to the fact that the graphene$h$-BN lattice mismatch leads to incommensurate structures.[62, 118] A recent experiment however shows a gap of $\sim 30 \mathrm{meV}$ in aligned graphene $\mid h$-BN structures,[139] and recent calculations show a gap in graphene of similar size in incommensurate, but aligned graphene $\mid h$-BN lattices.[130]

A question is to what extend the occurrence of a band gap would change the electron-hole puddles. Obviously if the gap is smaller than the variation in the potential $\Delta V(x, y)$ electron-hole puddles still form in qualitatively the same pattern as in the gapless state, as illustrated by Figure 9.7(bottom). Only in particular regions is the Fermi level in the gap, and the graphene sheet as a whole will still have a sizable conductivity. Only if the band gap is larger than the potential variation (and the 

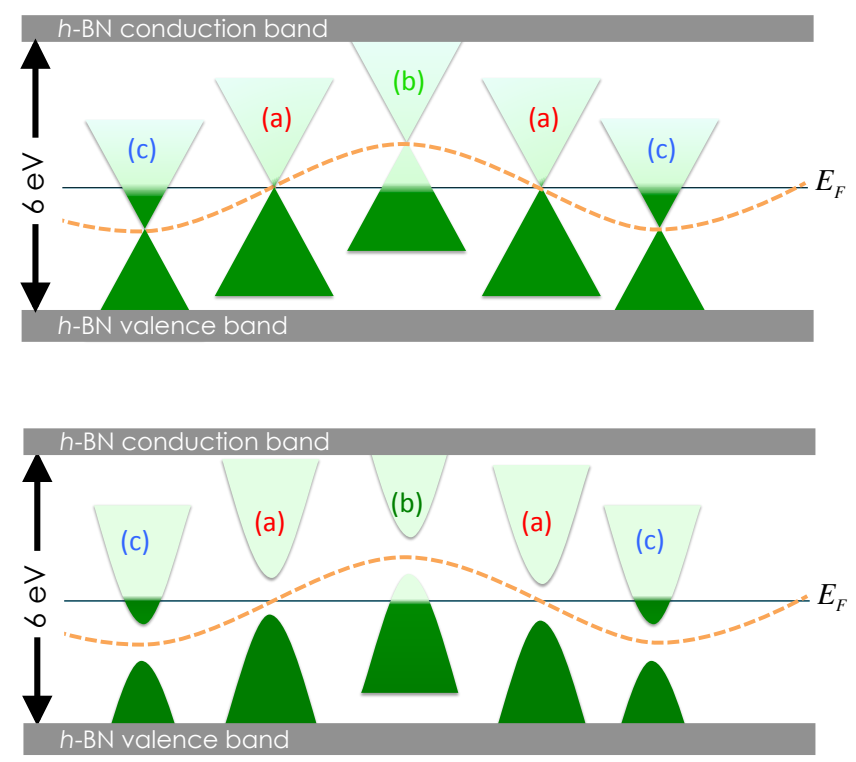

Figure 9.7: Schematic illustration of the influence of the potential landscape on the position of the Dirac cone. The potential $\Delta V(x, y)$ that modulates is indicated in by the (orange) dashed line. The top/bottom figures illustrate the formation of electronhole puddles without/with the opening of a graphene band gap.

Fermi level is in the gap) should the conductivity drop substantially.

\subsection{Graphene on Molybdenum Disulfide}

To broaden our study of the formation of electron-hole puddles, we also place graphene on molybdenum disulfide $\left(\mathrm{MoS}_{2}\right)$, which like $h$-BN is a layered insulating (semiconducting) material. The bonding between $\mathrm{MoS}_{2}$ layers is weak and the crystal can be cleaved in a similar fashion as $h-\mathrm{BN}$, which makes it possible to fabricate a perfect planar $\mathrm{MoS}_{2}$ substrate for graphene. $\mathrm{A} \mathrm{MoS}_{2}$ monolayer has a hexagonal structure with a layer of Mo atoms sandwiched between two layers of $S$ atoms, see Figure 9.8. The lattice mismatch with graphene is $\sim 22 \%$, which is much larger than the mismatch between graphene and $h$-BN.

We calculate the dipole and potential step at a graphene $\mid \mathrm{MoS}_{2}$ interface, using a $5 \times 5$ graphene on top of a $4 \times 4 \mathrm{MoS}_{2}$ in-plane supercell. As before, we fix the lattice constant of graphene to its optimized LDA value, see Table 9.1, and adapt the lattice constant of $\mathrm{MoS}_{2}$ accordingly. The LDA equilibrium binding distance $d_{\text {eq }}$ between the $\mathrm{MoS}_{2}$ monolayer and the graphene sheet is $3.34 \AA$. The calculated interface potential step, Eqs. (9.1), is $\Delta V=0.26 \mathrm{eV}$. This value is about three times larger than the maximum value we found for the graphene $\mid h$-BN interface, which implies that the charge displacement at the graphene $\mid \mathrm{MoS}_{2}$ interface is larger. However, upon trans- 


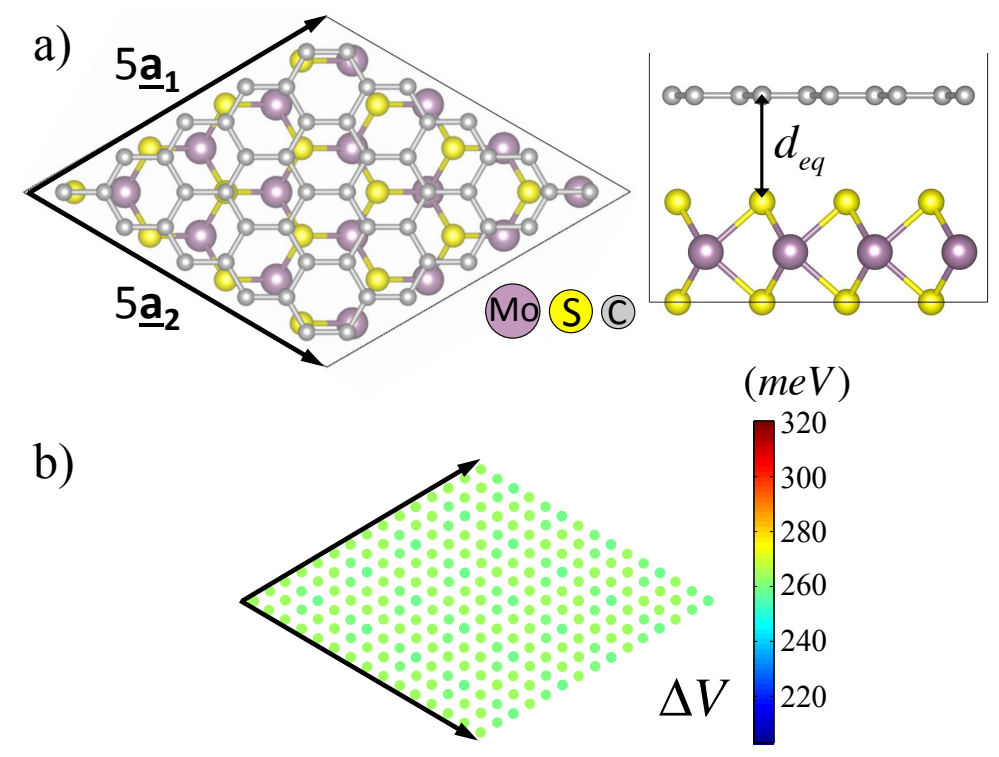

Figure 9.8: a) Graphene on Molybdenum Disulfide in a supercell consisting of $5 \times 5$ graphene on top of $4 \times 4 \mathrm{MoS}_{2}$ unit cells. Top/side views are shown on the left/right. b) The interface dipole step $\Delta V(x, y)$ as function of $x y$ translation of the graphene sheet over the $\mathrm{MoS}_{2}$ substrate at a constant graphene-MoS separation of $3.34 \AA$. The range of the energy scale is the same as in Fig. 9.3b).

lation of the graphene lattice over the $\mathrm{MoS}_{2}$ substrate, we find that the variation in the potential step $\Delta V(x, y)$, Eq. (9.2), is negligible. The results of these calculations are shown in Figure 9.8b), where we have used the same range in the energy scale as in Fig. 9.3b). The difference between the minimum and maximum $\Delta V$ is only 7 $\mathrm{meV}$, compared to $110 \mathrm{meV}$ for graphene on $h$-BN at similar distance.

The surface of a $\mathrm{MoS}_{2}$ monolayer consists of $\mathrm{S}$ atoms only, and it does not have in-plane polar bonds as $h$-BN has. One therefore expects the $\mathrm{MoS}_{2}$ surface not to present a strong local in-plane polarity to a graphene layer, as the $\mathrm{B}$ and $\mathrm{N}$ in $h$-BN do. Moreover, the large lattice mismatch between graphene and $\mathrm{MoS}_{2}$ gives a local averaging of the electrostatics. Therefore, we predict an absence of intrinsic electronhole puddles in graphene on $\mathrm{MoS}_{2}$.

\subsection{Conclusion}

In conclusion, we have found that an interface dipole layer is formed between graphene and $h$-BN. The size and direction of the accompanying potential step are dependent on the precise binding configuration. Since in experiment graphene on $h$-BN forms a incommensurable structure in which the local binding configuration gradually changes, we expect an slowly varying dipole layer and a corresponding electro- 
static potential landscape. Assuming that the length scale of this variation is large, the Dirac cone follows the local electrostatic potential profile. The result is a redistribution of charge in-plane, causing intrinsic electron-hole puddles to appear in graphene. For graphene placed on $\mathrm{MoS}_{2}$ the variation in the local electrostatic potential is negligible, and electron-hole puddles are not formed. 


\section{A}

\section{Graphene Brillouin zone sampling}

The first-principles calculations presented in chapter 5 were carried out with a $36 \times$ 36 sampling of the two-dimensional (2D) Brillouin zone (BZ) for $(1 \times 1)$ graphene or an equivalent sampling density for $(2 \times 2)$ graphene. At energies close to the charge neutrality level of graphene, the density of states (DoS) is described well by the linear expression $D(E)=D_{0}|E| / A$ with $A$ the surface area of a graphene unit cell. Table A.1 shows how the parameter $D_{0}$ depends on the number of k-points used to sample the 2D-BZ. An unexpectedly dense grid ( $288 \times 288$ points) is required to obtain a converged DoS which in view of the rather simple linear behavior of the DoS is at first glance surprising.

The main reason for requiring such a dense grid lies in the fact that for energies within $1 \mathrm{eV}$ of the Fermi level, all of the states contributing to the DoS lie in a very small part of the BZ around the conical points. This is illustrated in Fig. A.1 where it can be seen that even a $36 \times 36 \mathrm{k}$-point grid leads to a very coarse sampling of the

\begin{tabular}{rlclll}
\hline \multicolumn{4}{c}{$D_{0}$ states $/\left(\mathrm{eV}^{2}\right.$ unit cell) } \\
$N$ & $E<0$ & $E>0$ & $E<0$ & $E>0$ \\
\hline 36 & 0.088 & 0.092 & 0.102 & 0.102 \\
72 & 0.112 & 0.119 & 0.121 & 0.112 \\
144 & 0.109 & 0.116 & 0.114 & 0.115 \\
288 & 0.108 & 0.115 & 0.114 & 0.114 \\
\hline
\end{tabular}

Table A.1: Values of $D_{0}$ calculated using a grid obtained by dividing the reciprocal lattice vectors of the graphene lattice into $N$ intervals, and using the tetrahedron method[148] to calculate the DoS. All grids contain the K-point. 

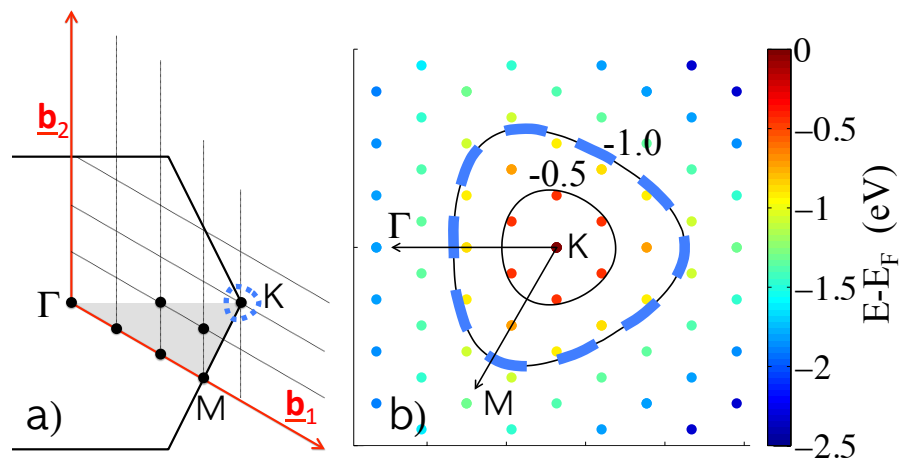

Figure A.1: a) Irreducible Brillouin zone (IBZ) of the graphene unit cell sampled with a $6 \times 6$ k-point grid. b) The eigenvalues of free-standing graphene in the vicinity of the Dirac (K) point sampled with a $36 \times 36 \mathrm{k}$-point grid. The dashed contour about the K point in a) and b) represent a cut through the Dirac "cone" at $-1.0 \mathrm{eV}$ from the Dirac point while the solid contour in $b$ ) represents a cut at $-0.5 \mathrm{eV}$.

relevant bands close to the conical points.

The DoS for various grid densities is shown in Figure A.2, calculated with the linear tetrahedron method[148], as implemented in VASP.[12, 44, 45] For the $36 \times 36$ grid the DoS is perfectly linear up to $\pm 0.45 \mathrm{eV}$, where it jumps to a different value. These jumps occur at eigenvalues corresponding to the k-points that are explicitly included in the grid. With increasing grid density the number of jumps increase, but their size decreases, such that the DoS converges to a linear function.

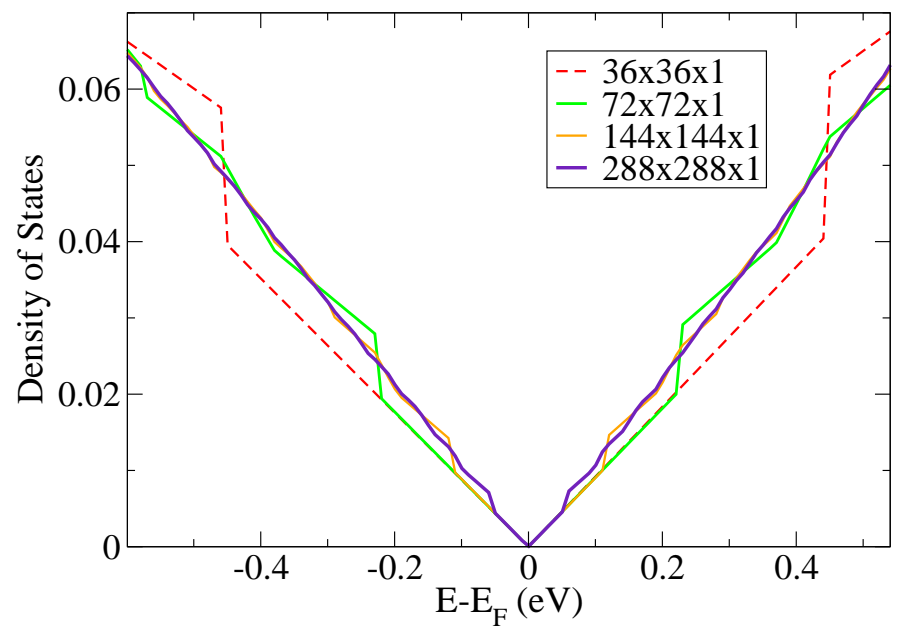

Figure A.2: DoS of graphene close to the Dirac point obtained by successively doubling the linear sampling density of the k-point grid used in the tetrahedron scheme. With increasing grid density the DoS converges to a smooth line. 


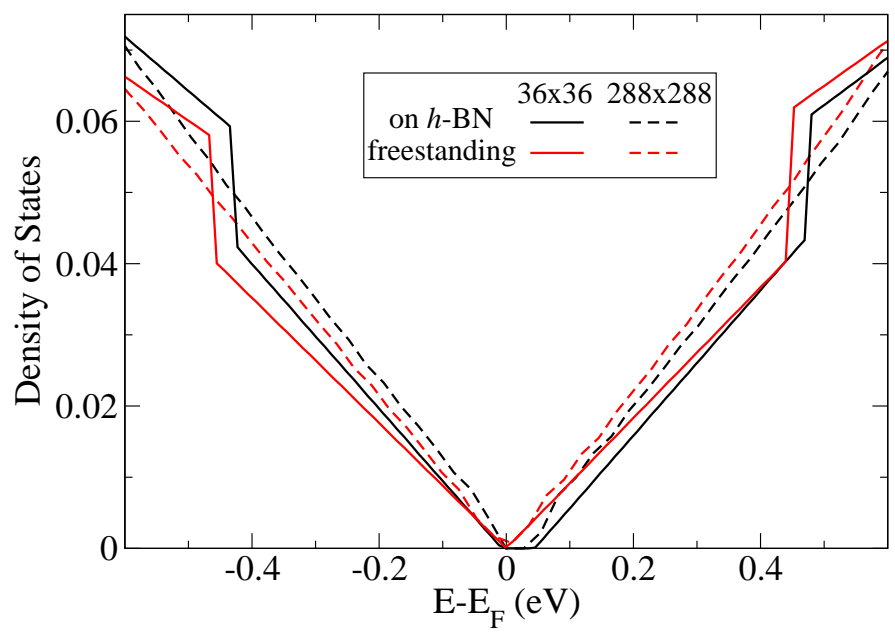

Figure A.3: DoS of free-standing graphene (red) and of graphene on $h$-BN (black), close to the Dirac point. The solid and dashed curves were calculated using $36 \times 36$ and $288 \times 288$ grids, respectively.

The jumps in the DoS at the k-grid points are not an effect of the trigonal distortion of the graphene Dirac cone; the DoS of an ideal conical dispersion $E \propto|k|$ (centered on $\Gamma$ ) shows similar jumps when calculated with the tetrahedron method. The slopes $D_{0}$ listed in Table A.1 were extracted by fitting a linear curve to the DoS for energies +0.4 (or -0.4$) \mathrm{eV}$ with respect to the charge neutrality energy. For freestanding graphene the converged LDA values are 0.108 and $0.115 /\left(\mathrm{eV}^{2}\right.$ unit cell) for the filled and empty states, respectively.

The graphene DoS also depends somewhat on the substrate used, as illustrated by Fig. A.3 and Table A.1. The slope $D_{0}$ for graphene adsorbed on $h$-BN is somewhat larger than that of free-standing graphene for holes $E<0$ and somewhat smaller for electrons $E<0$. Curiously, the DoS is more symmetric for electrons and holes for graphene on $h$-BN. A larger $D_{0}$ means that the graphene $\pi$-bands, and therefore the Dirac cone, become somewhat flattened, as illustrated in Fig. A.4.

The DFT calculations on $\mathrm{M}|\mathrm{BN}| \mathrm{Gr}$ structures reported in this manuscript were carried out using a $36 \times 36$ k-point grid. For internal consistency, a value $D_{0}=$ $0.102 /\left(\mathrm{eV}^{2}\right.$ unit cell) corresponding to this grid (see Table A.1) was used in the model that fits the numerical results essentially perfectly. 


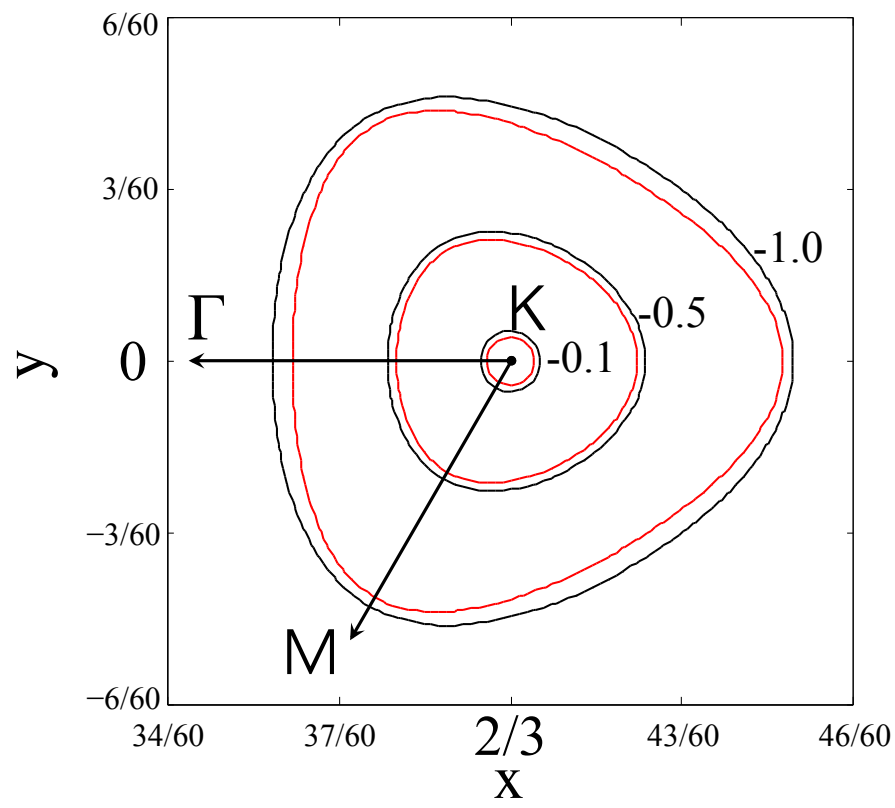

Figure A.4: Cross sections of the graphene Dirac cone at energies $-0.1,-0.5,-1.0 \mathrm{eV}$ with respect to the charge neutrality point, as calculated using a $288 \times 288 \mathrm{k}$-point grid. The black lines correspond to graphene on $h$ - $\mathrm{BN}$ and the red lines to freestanding graphene. The axes are in units of $2 \pi / a$. 


\section{Bibliography}

[1] K. S. Novoselov, A. K. Geim, S. V. Morozov, D. Jiang, Y. Zhang, S. V. Dubonos, I. V. Grigorieva, and A. A. Firsov, Electric Field Effect in Atomically Thin Carbon Films, Science 306, 666 (2004).

[2] P. C. Rusu and G. Brocks, Work functions of self-assembled monolayers on metal surfaces by first-principles calculations, Phys. Rev. B 74, 073414 (2006).

[3] P. R. Wallace, The Band Theory of Graphite, Phys. Rev. 71, 622 (1947).

[4] G. Giovannetti, P. A. Khomyakov, G. Brocks, P. J. Kelly, and J. van den Brink, Substrate-induced band gap in graphene on hexagonal boron nitride: Ab initio density functional calculations, Phys. Rev. B 76, 073103 (2007).

[5] B. Radisavljevic, A. Radenovic, J. Brivio, V. Giacometti, and A. Kis, Single-layer MoS2 transistors, Nature Nanotechnology 6, 147 (2011).

[6] P. Hohenberg and W. Kohn, Inhomogeneous Electron Gas, Phys. Rev. 136, B864 (1964).

[7] W. Kohn and L. J. Sham, Self-Consistent Equations Including Exchange and Correlation Effects, Phys. Rev. 140, A1133 (1965).

[8] R. O. M. Born, Zur Quantentheorie der Molekeln, Annalen der Physik 389, 457 (1927).

[9] R. M. Martin, "Electronic structure: Basic theory and practical methods," (Cambridge University Press, Cambridge, United Kingdom, 2004) pp. 42-44.

[10] M. C. Payne, M. P. Teter, D. C. Allan, T. A. Arias, and J. D. Joannopoulos, Iterative minimization techniques for ab initio total-energy calculations: molecular dynamics and conjugate gradients, Rev. Mod. Phys. 64, 1045 (1992).

[11] D. R. Hamann, M. Schluter, and C. Chiang, Norm-Conserving Pseudopotentials, Phys. Rev. Lett. 43, 1494 (1979).

[12] G. Kresse and D. Joubert, From ultrasoft pseudopotentials to the projector augmented-wave method, Phys. Rev. B 59, 1758 (1999). 
[13] D. Vanderbilt, Soft self-consistent pseudopotentials in a generalized eigenvalue formalism, Phys. Rev. B 41, 7892 (1990).

[14] P. E. Blöchl, Projector augmented-wave method, Phys. Rev. B 50, 17953 (1994).

[15] D. M. Ceperley and B. J. Alder, Ground State of the Electron Gas by a Stochastic Method, Phys. Rev. Lett. 45, 566 (1980).

[16] J. P. Perdew and A. Zunger, Self-interaction correction to density-functional approximations for many-electron systems, Phys. Rev. B 23, 5048 (1981).

[17] W. Kohn, Nobel Lecture: Electronic structure of matter: wave functions and density functionals, Rev. Mod. Phys. 71, 1253 (1999).

[18] M. S. Hybertsen and S. G. Louie, Electron correlation in semiconductors and insulators: Band gaps and quasiparticle energies, Phys. Rev. B 34, 5390 (1986).

[19] J. P. Perdew, J. A. Chevary, S. H. Vosko, K. A. Jackson, M. R. Pederson, D. J. Singh, and C. Fiolhais, Atoms, Molecules, Solids, and Surfaces - Applications of the Generalized Gradient Approximation for Exchange and Correlation, Phys. Rev. B 46, 6671 (1992).

[20] J. P. Perdew, K. Burke, and M. Ernzerhof, Generalized Gradient Approximation Made Simple, Phys. Rev. Lett. 77, 3865 (1996).

[21] J. Paier, M. Marsman, and G. Kresse, Why does the B3LYP hybrid functional fail for metals?, J. of Chem. Phys. 127, 024103 (2007).

[22] H. Rydberg, M. Dion, N. Jacobson, E. Schroder, P. Hyldgaard, S. I. Simak, D. C. Langreth, and B. I. Lundqvist, Van der Waals density functional for layered structures, Phys. Rev. Lett. 91, 126402 (2003).

[23] M. Dion, H. Rydberg, E. Schröder, D. C. Langreth, and B. I. Lundqvist, Van der Waals Density Functional for General Geometries, Phys. Rev. Lett. 92, 246401 (2004).

[24] L. Hedin, New Method for Calculating the One-Particle Green's Function with Application to the Electron-Gas Problem, Phys. Rev. 139, A796 (1965).

[25] M. S. Hybertsen and S. G. Louie, First-Principles Theory of Quasiparticles: Calculation of Band Gaps in Semiconductors and Insulators, Phys. Rev. Lett. 55, 1418 (1985).

[26] M. Shishkin and G. Kresse, Implementation and performance of the frequencydependent GW method within the PAW framework, Phys. Rev. B 74, 035101 (2006).

[27] H. Ishii, K. Sugiyama, E. Ito, and K. Seki, Energy Level Alignment and Interfacial Electronic Structures at Organic/Metal and Organic/Organic Interfaces, Adv. Mater. 11, 605 (1999). 
[28] A. Kahn, N. Koch, and W. Gao, Electronic Structure and Electrical Properties of Interfaces between Metals and $\pi$-Conjugated Molecular Films, Journal of Polymer Science: Part B: Polymer Physics 41, 2529 (2003).

[29] S. Braun, W. R. Salaneck, and M. Fahlman, Energy-Level Alignment at Organic/Metal and Organic/ Organic Interfaces, Adv. Mater. 21, 1450 (2009).

[30] G. Brocks, Polarons and bipolarons in oligothiophenes: a first-principles study, Synth. Met. 102, 914 (1999).

[31] G. Brocks, J. van den Brink, and A. F. Morpurgo, Electronic Correlations in Oligoacene and -Thiopene Organic Molecular Crystals, Phys. Rev. Lett. 93, 146405 (2004).

[32] H. Vázquez, R. Oszwaldowski, P. Pou, J. Ortega, R. Pérez, F. Flores, and A. Kahn, Dipole formation at metal/PTCDA interfaces: Role of the Charge Neutrality Level, Europhys. Lett. 65, 802 (2004).

[33] H. Vázquez, F. Flores, R. Oszwaldowski, J. Ortega, R. Pérez, and A. Kahn, Barrier formation at metalorganic interfaces: dipole formation and the charge neutrality level, Appl. Surf. Sci. 234, 107 (2004).

[34] L. Romaner, G. Heimel, J.-L. Brédas, A. Gerlach, F. Schreiber, R. L. Johnson, J. Zegenhagen, S. Duhm, N. Koch, and E. Zojer, Impact of Bidirectional Charge Transfer and Molecular Distortions on the Electronic Structure of a Metal-Organic Interface, Phys. Rev. Lett. 99, 256801 (2007).

[35] S. Duhm, G. Heimel, I. Salzmann, H. Glowatzki, R. L. Johnson, A. Vollmer, $\mathrm{J}$. Rabe, and N. Koch, Orientation-dependent ionization energies and interface dipoles in ordered molecular assemblies, Nature Mater. 7, 326 (2008).

[36] G. M. Rangger, O. T. Hofmann, L. Romaner, G. Heimel, B. Bröker, R.-P. Blum, R. L. Johnson, N. Koch, and E. Zojer, F4TCNQ on Cu, $A g$, and $A u$ as prototypical example for a strong organic acceptor on coinage metals, Phys. Rev. B 79, 165306 (2009).

[37] P. C. Rusu, G. Giovannetti, C. Weijtens, R. Coehoorn, and G. Brocks, Work Function Pinning at Metal-Organic Interfaces, J. Phys. Chem. C 113, 9974 (2009).

[38] P. C. Rusu, G. Giovannetti, C. Weijtens, R. Coehoorn, and G. Brocks, Firstprinciples study of the dipole layer formation at metal-organic interfaces, Phys. Rev. B 81, 125403 (2010).

[39] L. Vitali, G. Levita, R. Ohmann, A. Comisso, A. D. Vita, and K. Kern, Portrait of the potential barrier at metalorganic nanocontacts, Nature Mat. 9, 320 (2010).

[40] T.-C. Tseng, C. Urban, Y. Wang, R. Otero, S. L. Tait, M. Alcamí, D. Écija, M. Trelka, J. M. Gallego, N. Lin, M. Konuma, U. Starke, A. Nefedov, A. Langner, C. Wöll, M. Á. Herranz, F. Mart'n, N. Mart'n, K. Kern, and 
R. Miranda, Charge-transfer-induced structural rearrangements at both sides of organic/metal interfaces, Nature Chem. 2, 374 (2010).

[41] W. Osikowicz, M. P. de Jong, and W. R. Salaneck, Formation of the interfacial dipole at organic-organic interfaces: C-60/polymer interfaces, Adv. Mater. 19, 4213 (2007).

[42] O. Gunnarsson, Superconductivity in fullerides, Rev. Mod. Phys. 69, 575 (1997).

[43] D. Cahen and A. Kahn, Electron Energetics at Surfaces and Interfaces: Concepts and Experiments, Adv. Mater. 15, 271 (2003).

[44] G. Kresse and J. Hafner, Ab-initio Molecular-Dynamics for Liquid-Metals, Phys. Rev. B 47, 558 (1993).

[45] G. Kresse and J. Furthmüller, Efficient iterative schemes for ab initio total-energy calculations using a plane-wave basis set, Phys. Rev. B 54, 11169 (1996).

[46] S. Braun and W. R. Salaneck, Fermi level pinning at interfaces with tetrafluorotetracyanoquinodimethane (F4-TCNQ): The role of integer charge transfer states, Chem. Phys. Lett. 438, 259 (2007).

[47] R. J. Murdey and W. R. Salaneck, Charge injection barrier heights across multilayer organic thin films, Jpn. J. Appl. Phys. Part 1 44, 3751 (2005).

[48] I. Hill, D. Milliron, J. Schwartz, and A. Kahn, Organic semiconductor interfaces: electronic structure and transport properties, Appl. Surf. Sci. 166, 354 (2000).

[49] Y. Tanaka, K. Kanai, Y. Ouchi, and K. Seki, Role of interfacial dipole layer for energy-level alignment at organic/metal interfaces, Org. Electron. 10, 990 (2009).

[50] H. Peisert, A. Petr, L. Dunsch, T. Chassé, and M. Knupfer, Interface Fermi Level Pinning at Contacts Between PEDOT:PSS and Molecular Organic Semiconductors, ChemPhysChem 8, 386 (2007).

[51] G. Giovannetti, G. Brocks, and J. van den Brink, Ab initio electronic structure and correlations in pristine and potassium-doped molecular crystals of copper phthalocyanine, Phys. Rev. B 77, 035133 (2008).

[52] A. K. Geim and K. S. Novoselov, The rise of graphene, Nature Materials 6, 183 (2007).

[53] K. S. Novoselov, A. K. Geim, S. V. Morozov, D. Jiang, M. I. Katsnelson, I. V. Grigorieva, S. V. Dubonos, and A. A. Firsov, Two-dimensional gas of massless Dirac fermions in graphene, Nature 438, 197 (2005).

[54] Y. B. Zhang, Y. W. Tan, H. L. Stormer, and P. Kim, Experimental observation of the quantum Hall effect and Berry's phase in graphene, Nature 438, 201 (2005). 
[55] M. Ishigami, J. H. Chen, W. G. Cullen, M. S. Fuhrer, and E. D. Williams, Atomic structure of graphene on $\mathrm{SiO}_{2}$, Nano Letters 7, 1643 (2007).

[56] E. Stolyarova, K. T. Rim, S. Ryu, J. Maultzsch, P. Kim, L. E. Brus, T. F. Heinz, M. S. Hybertsen, and G. W. Flynn, High-resolution scanning tunneling microscopy imaging of mesoscopic graphene sheets on an insulating surface, Proc. Natl. Acad. Sci. U.S.A. 104, 9209 (2007).

[57] J.-H. Chen, C. Jang, S. Adam, M. S. Fuhrer, E. D. Williams, and M. Ishigami, Charged-impurity scattering in graphene, Nature Physics 4, 377 (2008).

[58] Y. B. Zhang, V. W. Brar, C. Girit, A. Zettl, and M. F. Crommie, Origin of spatial charge inhomogeneity in graphene, Nature Physics 5, 722 (2009).

[59] J. Martin, N. Akerman, G. Ulbricht, T. Lohmann, J. H. Smet, K. von Klitzing, and A. Yacoby, Observation of electron-hole puddles in graphene using a scanning single-electron transistor, Nature Physics 4, 144 (2008).

[60] K. Watanabe, T. Taniguchi, and H. Kanda, Direct-bandgap properties and evidence for ultraviolet lasing of hexagonal boron nitride single crystal, Nature Materials 3, 404 (2004).

[61] C. R. Dean, A. F. Young, I. Meric, C. Lee, L. Wang, S. Sorgenfrei, K. Watanabe, T. Taniguchi, P. Kim, K. L. Shepard, and J. Hone, Boron nitride substrates for high-quality graphene electronics, Nature Nanotechnology 5, 722 (2010).

[62] J. Xue, J. Sanchez-Yamagishi, D. Bulmash, P. Jacquod, A. Deshpande, K. Watanabe, T. Taniguchi, P. Jarillo-Herrero, and B. J. LeRoy, Scanning tunnelling microscopy and specroscopy of ultra-flat graphene on hexagonal boron nitride, Nature Materials 10, 282 (2011).

[63] R. Decker, Y. Wang, V. W. Brar, W. Regan, H.-Z. Tsai, Q. Wu, W. Gannett, A. Zettl, and M. F. Crommie, Local Electronic Properties of Graphene on a BN Substrate via Scanning Tunneling Microscopy, Nano Letters 11, 2291 (2011).

[64] K. S. Novoselov, D. Jiang, F. Schedin, T. J. Booth, V. V. Khotkevich, S. V. Morozov, and A. K. Geim, Two-dimensional atomic crystals, Proc. Natl. Acad. Sci. U.S.A. 102, 10451 (2005).

[65] J. C. Meyer, A. Chuvilin, G. Algara-Siller, J. Biskupek, and U. Kaiser, Selective sputtering and atomic resolution imaging of atomically thin boron nitride membranes, Nano Letters 9, 2683 (2009).

[66] L. Song, L. Ci, H. Lu, P. B. Sorokin, C. Jin, J. Ni, A. G. Kvashnin, D. G. Kvashnin, J. Lou, B. I. Yakobson, and P. M. Ajayan, Large scale growth and characterization of atomic hexagonal boron nitride layers, Nano Letters 10, 3209 (2010). 
[67] Y. Shi, C. Hamsen, X. Jia, K. K. Kim, A. Reina, M. Hofmann, A. L. Hsu, K. Zhang, H. Li, Z.-Y. Juang, M. S. Dresselhaus, L.-J. Li, and J. Kong, Synthesis of few-layer hexagonal boron nitride thin film by chemical vapor deposition, Nanoletters 10, 4134 (2010).

[68] D. Usachov, V. K. Adamchuk, D. Haberer, A. Grueneis, H. Sachdev, A. B. Preobrajenski, C. Laubschat, and D. V. Vyalikh, Quasifreestanding single-layer hexagonal boron nitride as a substrate for graphene synthesis, Phys. Rev. B 82, 075415 (2010).

[69] Y. B. Zhang, V. W. Brar, F. Wang, C. Girit, Y. Yayon, M. Panlasigui, A. Zettl, and M. F. Crommie, Giant phonon-induced conductance in scanning tunnelling spectroscopy of gate-tunable graphene, Nature Physics 4, 627 (2008).

[70] Y. J. Yu, Y. Zhao, S. Ryu, L. E. Brus, K. S. Kim, and P. Kim, Tuning the Graphene Work Function by Electric Field Effect, Nano Letters 9, 3430 (2009).

[71] V. M. Karpan, G. Giovannetti, P. A. Khomyakov, M. Talanana, A. A. Starikov, M. Zwierzycki, J. van den Brink, G. Brocks, and P. J. Kelly, Graphite and graphene as perfect spin filters, Phys. Rev. Lett. 99, 176602 (2007).

[72] G. Giovannetti, P. A. Khomyakov, G. Brocks, V. M. Karpan, J. van den Brink, and P. J. Kelly, Doping graphene with metal contacts, Phys. Rev. Lett. 101, 026803 (2008).

[73] P. A. Khomyakov, G. Giovannetti, P. C. Rusu, G. Brocks, J. van den Brink, and P. J. Kelly, First-principles study of the interaction and charge transfer between graphene and metals, Phys. Rev. B 79, 195425 (2009).

[74] P. C. Rusu, G. Giovannetti, C. Weijtens, R. Coehoorn, and G. Brocks, Work Function Pinning at Metal-Organic Interfaces, J. Phys. Chem. C 113, 9974 (2009).

[75] P. C. Rusu, G. Giovannetti, C. Weijtens, R. Coehoorn, and G. Brocks, Firstprinciples study of the dipole layer formation at metal-organic interfaces, Phys. Rev. B 81, 125403 (2010).

[76] P. A. Khomyakov, A. A. Starikov, G. Brocks, and P. J. Kelly, Nonlinear screening of charges induced in graphene by metal contacts, Phys. Rev. B 82, 115437 (2010).

[77] E. J. H. Lee, K. Balasubramanian, R. T. Weitz, M. Burghard, and K. Kern, Contact and edge effects in graphene devices, Nature Nanotechnology 3, 486 (2008).

[78] Y. Wu, V. Perebeinos, Y. Lin, T. Low, F. Xia, and P. Avouris, Quantum Behavior of Graphene Transistors near the Scaling Limit, Nano Letters 12, 1417 (2012).

[79] B. Huard, N. Stander, J. A. Sulpizio, and D. Goldhaber-Gordon, Evidence of the role of contacts on the observed electron-hole asymmetry in graphene, Phys. Rev. B 78, 121402 (2008). 
[80] L. Britnell, R. V. Gorbachev, R. Jalil, B. D. Belle, F. Schedin, A. Mishchenko, T. Georgiou, M. I. Katsnelson, L. Eaves, S. V. Morozov, N. M. R. Peres, J. Leist, A. K. Geim, K. S. Novoselov, and L. A. Ponomarenko, Field-Effect Tunneling Transistor Based on Vertical Graphene Heterostructures, Science 335, 947 (2012).

[81] K. I. Bolotin, K. J. Sikes, Z. Jiang, G. Fudenberg, J. Hone, P. Kim, and H. L. Stormer, Ultrahigh electron mobility in suspended graphene, Sol. State Comm. 146, 351 (2008).

[82] V. M. Karpan, P. A. Khomyakov, G. Giovannetti, A. A. Starikov, and P. J. Kelly, Ni(111)|graphene|h-BN junctions as ideal spin injectors, Phys. Rev. B 84, 153406 (2011).

[83] M. Bokdam, P. A. Khomyakov, G. Brocks, Z. Zhong, and P. J. Kelly, Electrostatic doping of graphene through ultrathin hexagonal boron nitride films, Nano Letters 11, 4631 (2011).

[84] G.-H. Lee, Y.-J. Yu, C. Lee, C. Dean, K. L. Shepard, P. Kim, and J. Hone, Electron tunneling through atomically flat and ultrathin hexagonal boron nitride, Appl. Phys. Lett. 99, 243114 (2011).

[85] L. Britnell, R. V. Gorbachev, R. Jalil, B. D. Belle, F. Schedin, M. I. Katsnelson, L. Eaves, S. V. Morozov, A. S. Mayorov, N. M. R. Peres, A. H. C. Neto, J. Leist, A. K. Geim, L. A. Ponomarenko, and K. S. Novoselov, Electron Tunneling through Ultrathin Boron Nitride Crystalline Barriers, Nano Letters 12, 1707 (2012).

[86] T. Greber, M. Corso, and J. Osterwalder, Fermi surfaces of single layer dielectrics on transition metals, Surface Science 603, 1373 (2009).

[87] A. B. Preobrajenski, A. S. Vinogradov, and N. Mårtensson, Monolayer of h$B N$ chemisorbed on $\mathrm{Cu}(111)$ and $\mathrm{Ni}(111)$ : The role of the transition metal $3 d$ states, Surface Science 582, 21 (2005).

[88] E. Ćavar, R. Westerström, A. Mikkelsen, E. Lundgren, A. S. Vinogradov, M. L. $\mathrm{Ng}$, A. B. Preobrajenski, A. A. Zakharov, and N. Mårtensson, $A$ single h-BN layer on Pt(111), Surface Science 602, 1722 (2008).

[89] C. Oshima, A. Itoh, E. Rokuta, T. Tanaka, K. Yamashita, and T. Sakurai, A hetero-epitaxial-double-atomic-layer system of monolayer graphene/monolayer $h-B N$ on Ni(111), Sol. State Comm. 116, 37 (2000).

[90] C. Bjelkevig, Z. Mi, J. Xiao, P. A. Dowben, L. Wang, W.-N. Mei, and J. A. Kelber, Electronic structure of a graphene/hexagonal-BN heterostructure grown on $R u(0001)$ by chemical vapor deposition and atomic layer deposition: extrinsically doped graphene, J. Phys.: Condens. Matter 22, 302002 (2010). 
[91] J. Neugebauer and M. Scheffler, Adsorbate-substrate and adsorbate-adsorbate interactions of na and $k$ adlayers on al(111), Phys. Rev. B 46, 16067 (1992).

[92] M. Methfessel and A. T. Paxton, High-precision sampling for Brillouin-zone integration in metals, Phys. Rev. B 40, 3616 (1989).

[93] R. Resta and K. Kunc, Self-consistent theory of electronic states and dielectric response in semiconductors, Phys. Rev. B 34, 7146 (1986).

[94] M. Yankowitz, J. Xue, D. Cormode, J. D. Sanchez-Yamagishi, K. Watanabe, T. Taniguchi, P. Jarillo-Herrero, P. Jacquod, and B. J. LeRoy, Emergence of superlattice Dirac points in graphene on hexagonal boron nitride, Nature Physics 8 , 382 (2012).

[95] T. A. Pham, T. Li, S. Shankar, F. Gygi, and G. Galli, First-principles investigations of the dielectric properties of crystalline and amorphous $S_{3} N_{4}$ thin films, Appl. Phys. Lett. 96, 062902 (2010).

[96] P. S. Bagus, V. Staemmler, and C. Wöll, Exchangelike Effects for Closed-Shell Adsorbates: Interface Dipole andWork Function, Phys. Rev. Lett. 89, 096104 (2002).

[97] S. Joshi, D. Ecija, R. Koitz, M. Iannuzzi, A. P. Seitsonen, J. Hutter, H. Sachdev, S. Vijayaraghavan, F. Bischoff, K. Seufert, J. V. Barth, and W. Auwärter, Boron Nitride on Cu(111): An Electronically Corrugated Monolayer, Nano Letters 12, 5821 (2012).

[98] A. Nagashima, N. Tejima, Y. Gamou, T. Kawai, and C. Oshima, Electronic states of monolayer hexagonal boron nitride formed on the metal surfaces, Surface Science 357-358, 307 (1996).

[99] G. B. Grad, P. Blaha, K. Schwarz, W. Auwärter, and T. Greber, Density functional theory investigation of the geometric and spintronic structure of $\mathrm{h}-\mathrm{BN} \mid \mathrm{Ni}(111)$ in view of photoemission and STM experiments, Phys. Rev. B 68, 085404 (2003).

[100] D. Leuenberger, H. Yanagisawa, S. Roth, J. Osterwalder, and M. Hengsberger, Disentanglement of electron dynamics and space-charge effects in time-resolved photoemission from $h-B N / N i(111)$, Phys. Rev. B 84, 125107 (2011).

[101] D. Otálvaro, T. Veening, and G. Brocks, Self-Assembled Monolayer Induced $A u(111)$ and $A g(111)$ Reconstructions: Work Functions and Interface Dipole Formation, J. Phys. Chem. C 116, 7826 (2012).

[102] M. Bokdam, D. Çakir, and G. Brocks, Fermi level pinning by integer charge transfer at electrode-organic semiconductor interfaces, Appl. Phys. Lett. 98, 113303 (2011).

[103] J. Wintterlin and M. L. Bocquet, Graphene on metal surfaces, Surface Science 603, 1841 (2009). 
[104] T. Georgiou, R. Jalil, B. D. Belle, L. Britnell, R. V. Gorbachev, S. V. Morozov, Y.-J. Kim, A. Gholinia, S. J. Haigh, O. Makarovsky, L. Eaves, L. A. Ponomarenko, A. K. Geim, K. S. Novoselov, and A. Mishchenko, Vertical Field Effect Transistor based on Graphene-WS ${ }_{2}$ Heterostructures for flexible and transparent electronics, arXiv:1211.5090 (2012).

[105] M. Bokdam, P. A. Khomyakov, G. Brocks, and P. J. Kelly, Field effect doping of graphene in metal—dielectric — graphene heterostructures: A model based upon firstprinciples calculations, Phys. Rev. B 87, 075414 (2013).

[106] G. Brocks and A. van der Avoird, Infrared Spectra of the van der Waals Molecule $\left(\mathrm{N}_{2}\right)_{2}$, Mol. Phys. 55, 11 (1985).

[107] G. Brocks, J. Tennyson, and A. van der Avoird, Ab Initio Dipole Surfaces, Vibrationally Averaged Dipole Moments, and Infrared Transition Intensities for KCN and LiCN, J. Chem. Phys. 80, 3223 (1984).

[108] M. J. Dresser, T. E. Madey, and J. T. Yates, The Adsorption of Xenon by W(111), and its Interaction with Preadsorbed Oxygen, Surface Science 42, 533 (1974).

[109] H. Vazquez, Y. J. Dappe, J. Ortega, and F. Flores, Energy level alignment at metal/organic semiconductor interfaces: "Pillow" effect, induced density of interface states, and charge neutrality level, J. Chem. Phys. 126, 144703 (2007).

[110] L. Vitali, G. Levita, R. Ohmann, A. Comisso, A. D. Vita, and K. Kern, Portrait of the Potential Barrier at Metal-Organic Nanocontacts, Nature Materials 9, 320 (2010).

[111] J. Gebhardt, F. Viñes, and A. Görling, Influence of the surface dipole layer and Pauli repulsion on band energies and doping in graphene adsorbed on metal surfaces, Phys. Rev. B 86, 195431 (2012).

[112] T. Thonhauser, V. R. Cooper, S. Li, A. Puzder, P. Hyldgaard, and D. C. Langreth, Van der Waals density functional: Self-consistent potential and the nature of the van der Waals bond, Phys. Rev. B 76, 125112 (2007).

[113] J. c. v. Klimeš, D. R. Bowler, and A. Michaelides, Van der Waals density functionals applied to solids, Phys. Rev. B 83, 195131 (2011).

[114] M. Reguzzoni, A. Fasolino, E. Molinari, and M. C. Righi, Potential energy surface for graphene on graphene: Ab initio derivation, analytical description, and microscopic interpretation, Phys. Rev. B 86, 245434 (2012).

[115] K. Berland and P. Hyldgaard, Analysis of van der Waals density functional components: Binding and corrugation of benzene and $C_{60}$ on boron nitride and graphene, Phys. Rev. B 87, 205421 (2013). 
[116] S. Roth, F. Matsui, T. Greber, and J. Osterwalder, Chemical Vapor Deposition and Characterization of Aligned and Incommensurate Graphene/Hexagonal Boron Nitride Heterostack on $\mathrm{Cu}$ (111), Nano Letters 13, 2668 (2013).

[117] K. P. Loh, I. Sakaguchi, M. N. Gamo, S. Tagawa, T. Sugino, and T. Ando, Surface conditioning of chemical vapor deposited hexagonal boron nitride film for negative electron affinity, Appl. Phys. Lett. 74, 28 (1999).

[118] B. Sachs, T. O. Wehling, M. I. Katsnelson, and A. I. Lichtenstein, Adhesion and electronic structure of graphene on hexagonal boron nitride substrates, Phys. Rev. B 84, 195414 (2011).

[119] R. Laskowski, P. Blaha, and K. Schwarz, Bonding of hexagonal BN to transition metal surfaces: An ab initio density-functional theory study, Phys. Rev. B 78, 045409 (2008).

[120] T. Olsen, J. Yan, J. J. Mortensen, and K. S. Thygesen, Dispersive and Covalent Interactions between Graphene and Metal Surfaces from the Random Phase Approximation, Phys. Rev. Lett. 107, 156401 (2011).

[121] E. Hazrati, Complex Hydrides for Hydrogen Storage: A First-Principles Study, Ph.D. thesis, Radboud University Nijmegen, The Netherlands (2013).

[122] R. W. Lynch and H. G. Drickamer, Effect of High Pressure on the Lattice Parameters of Diamond, Graphite, and Hexagonal Boron Nitride, J. Chem. Phys. 44, 181 (1966).

[123] R. V. Wang, D. D. Fong, F. Jiang, M. J. Highland, P. H. Fuoss, C. Thompson, A. M. Kolpak, J. A. Eastman, S. K. Streiffer, A. M. Rappe, and G. B. Stephenson, Reversible Chemical Switching of a Ferroelectric Film, Phys. Rev. Lett. 102, 047601 (2009).

[124] M. N. Huda and L. Kleinman, h-BN monolayer adsorption on the Ni (111) surface: A density functional study, Phys. Rev. B 74, 075418 (2006).

[125] T. Vaara, J. Vaari, and J. Lahtinen, Adsorption of Potassium on Co(0001), Surface Science 395, 88 (1993).

[126] H. B. Michaelson, The work function of the elements and its periodicity, J. Appl. Phys. 48, 4729 (1977).

[127] G. N. Derry and Z. Ji-Zhong, Work function of Pt(111), Phys. Rev. B 39, 1940 (1989).

[128] A. Nagashima, N. Tejima, Y. Gamou, T. Kawai, and C. Oshima, Electronic Structure of Monolayer Hexagonal Boron Nitride Physisorbed on Metal Surfaces, Phys. Rev. Lett. 75, 3918 (1995). 
[129] F. Müller, S. Hüfner, H. Sachdev, R. Laskowski, P. Blaha, and K. Schwarz, Epitaxial growth of hexagonal boron nitride on Ag(111), Phys. Rev. B 82, 113406 (2010).

[130] M. Bokdam, Charge transfer and redistribution at interfaces between metals and $2 D$ materials, Ph.D. thesis, University of Twente, The Netherlands (2013).

[131] T. Ando and T. Nakanishi, Impurity scattering in carbon nanotubes - Absence of back scattering, J. Phys. Soc. Jpn. 67, 1704 (1998).

[132] C.-H. Park, L. Yang, Y.-W. Son, M. L. Cohen, and S. G. Louie, Anisotropic behaviours of massless Dirac fermions in graphene under periodic potentials, Nature Physics 4, 213 (2008).

[133] M. Barbier, F. M. Peeters, P. Vasilopoulos, and J. M. Pereira, Jr., Dirac and KleinGordon particles in one-dimensional periodic potentials, Phys. Rev. B 77, 115446 (2008).

[134] C.-H. Park, L. Yang, Y.-W. Son, M. L. Cohen, and S. G. Louie, New Generation of Massless Dirac Fermions in Graphene under External Periodic Potentials, Phys. Rev. Lett. 101, 126804 (2008).

[135] P. Burset, A. L. Yeyat, L. Brey, and H. A. Fertig, Transport in superlattices on single-layer graphene, Phys. Rev. B 83, 195434 (2011).

[136] M. Kindermann, B. Uchoa, and D. L. Miller, Zero-energy modes and gate-tunable gap in graphene on hexagonal boron nitride, Phys. Rev. B 86, 115415 (2012).

[137] L. A. Ponomarenko, A. K. Geim, A. A. Zhukov, R. Jalil, S. V. Morozov, K. S. Novoselov, I. V. Grigorieva, E. H. Hill, V. V. Cheianov, V. I. Fal'ko, K.Watanabe, T. Taniguchi, and R. V. Gorbachev, Tunable metalinsulator transition in doublelayer graphene heterostructures, Nature Physics 7, 958 (2011).

[138] F. Amet, J. R. Williams, K.Watanabe, T.Taniguchi, and D. Goldhaber-Gordon, Insulating behavior at the neutrality point in single-layer graphene, Phys. Rev. Lett. 110, 216601 (2013).

[139] B. Hunt, J. D. Sanchez-Yamagishi, A. F. Young, K. Watanabe, T. Taniguchi, P. Moon, M. Koshino, P. Jarillo-Herrero, and R. C. Ashoori, Massive Dirac fermions and Hofstadter butterfly in a van der Waals heterostructure, Science 340, 1427 (2013).

[140] J. C. W. Song, A. V. Shytov, and L. S. Levitov, Engineering Interaction Effects and Gap Opening in Graphene Superlattices, arXiv:1212.6759v3 (2013).

[141] N. Berseneva, A. Gulans, A. V. Krasheninnikov, and R. M. Nieminen, Electronic structure of boron nitride sheets doped with carbon from first-principles calculations, Phys. Rev. B 87, 035404 (2013). 
[142] F. Hüser, T. Olsen, and K. S. Thygesen, Quasiparticle GW calculations for solids, molecules, and two-dimensional materials, Phys. Rev. B 87, 235132 (2013).

[143] T. Ando, Screening Effect and Impurity Scattering in Monolayer Graphene, J. Phys. Soc. Jpn. 75, 074716 (2006).

[144] V. V. Cheianov and V. I. Fal'ko, Friedel Oscillations, Impurity Scattering, and Temperature Dependence of Resistivity in Graphene, Phys. Rev. Lett. 97, 226801 (2006).

[145] K. Nomura and A. H. MacDonald, Quantum Hall Ferromagnetism in Graphene, Phys. Rev. Lett. 96, 256602 (2006).

[146] M. Gibertini, A. Tomadin, F. Guinea, M. I. Katsnelson, and M. Polini, Electronhole puddles in the absence of charged impurities, Phys. Rev. B 85, 201405 (2012).

[147] G. Kresse and J. Furthmüller, Efficiency of ab-initio total energy calculations for metals and semiconductors using a plane-wave basis set, Comp. Mat. Sci. 6, 15 (1996).

[148] P. E. Blöchl, O. Jepsen, and O. K. Andersen, Improved Tetrahedron Method for Brillouin-Zone Integrations, Phys. Rev. B 49, 16223 (1994).

[149] K. Momma and F. Izumi, VESTA: a three-dimensional visualization system for electronic and structural analysis, J. Appl. Crystallogr 41, 653 (2008). 


\section{Summary}

The experimental proof that a single graphene layer is stable in 2004 has led to a new HYPE*. The prospect of HighlY Planar Electronics is an appealing one for Nanotechnology: a conducting, one atom thick carbon sheet with peculiar electronic characteristics, as one of the primary building blocks for very thin planar electronic components. One can dream of resistors, transistors, diodes and planar capacitors consisting of graphene in combination with other 2D materials. Considering that these components would be extremely thin and their in-plane $x y$-dimensions much larger than their $z$-dimension, it is not strange to assume that the interfaces between the different materials when stacked in a multilayer would be very important. In this thesis the physical processes occurring at these interfaces are studied. The primary method used to study these systems is Density Functional Theory (DFT); how it works and what can be calculated using it are explained in chapter 2. The second method is to develop phenomenological models that give a simplified picture to interpret the results of the DFT calculations. With these models the interplay of the different processes occurring at the same time can then be studied.

We start by looking at metal|organic interfaces in chapter 3. In experimental studies constant work function levels (pinning levels) are measured after depositing the same molecules on different metal surfaces. The transfer of electrons between the metal surface and the molecular layer affects the work function of the moleculecovered surface. Whether electrons are transferred from the molecules to the metal or the other way around is determined by the charging energetics of the molecules and by the work function of the metal surface. A model based on a planar capacitor is constructed and together with DFT calculations is able to accurately predict pinning levels.

Chapter 4 proposes a graphene field effect device: a graphene|hexagonal BoronNitride $(h-\mathrm{BN})$ heterostructure on a copper surface. The charge transfer between the graphene sheet and the copper surface through the ultra-thin $h-\mathrm{BN}$ film is studied. Even though the pristine work function of copper is larger then that of graphene, electrons are still transferred form copper to graphene. This results in a graphene sheet that is intrinsically $n$-type doped. It is found that a considerable dipole layer is formed at the $\mathrm{Cu}(111) \mid h$ - $\mathrm{BN}$ interface and that this makes the effective work function

*A fitting abbreviation that my promotor Prof. Kelly came up with. 
of the copper surface smaller than that of graphene. The doping can be reversed or increased by applying an electric field over the structure. By modeling the system as a planar capacitor, we are able to calculate the doping level in the graphene sheet. Introducing terms accounting for the interfaces in the heterostructure results in a model that accurately fits the DFT-calculated doping level. In chapter 5 the copper surface is replaced by other transition metals. It is found that the potential step corresponding to the metal $\mid h$-BN dipole layer is considerable and different for all the metals studied here.

In chapter 6 we take a deeper look at the origins of the large potential steps occurring at weakly interacting metal|insulator interfaces. The metal $\mid h-\mathrm{BN}$ structure is used as an archetypal example and test case. The dipole layer formed at these interfaces is commonly explained in terms of Pauli exchange repulsion, sometimes referred to as the Pillow effect. To test this, a system is constructed from the antisymmetrized product of the metal and $h$-BN wave functions. This system then has exact Pauli exchange repulsion. At distances between the metal and the insulator surfaces that are typical for physisorption, exchange repulsion forms the main contribution to the interface potential step. At these and larger distances, chemical/van der Waals interactions significantly modify the local electron density, but make a relatively small contribution to the potential step. At shorter distances, chemical bonding interactions reduce the interface potential step.

The large potential steps found at the metal $\mid h-\mathrm{BN}$ interface are important for the energy level alignment in metal|2D material heterostructures. In chapter 7 the Schottky barriers at metal $\mid h-\mathrm{BN}$ are calculated by DFT. The barrier can be modeled by aligning the metal and $h$-BN densities of states to the vacuum level and then shifting the two apart with a potential step. The dependence of the potential step on the metal- $h$-BN separation is almost the same for all metals on which $h$-BN physisorbs. Therefore it is fitted by a single function, which can be used for all metals considered in this chapter.

In the last two chapters the interface effects at the interfaces between weakly bonded 2D materials are studied. In chapter 8 graphene is placed on a surface of $h$ $\mathrm{BN}$, which has a similar planar crystal structure with a slightly $(\sim 1.8 \%)$ larger lattice constant. Because of the weakness of the van der Waals bonding between these two layers, the structures do not become commensurate. The cost of straining the graphene or $h$-BN sheet to match the lattice constants outweighs the energy gain of bonding. As a result moiré patterns are observed in STM experiments, indicating a much larger supercell periodicity. The size of these supercells is determined by the angle between the graphene and $h$-BN lattice and the lattice mismatch. A realistic supercell is so large that it is beyond the capabilities of DFT methods implemented in present day supercomputers. Therefore a tight-binding model was constructed, which is parameterized to DFT results for commensurable systems. GW calculations are performed to determine the effect of many body interactions on the band gap induced in graphene by commensurate $h$-BN. The effect is huge, the GW gaps are approximately $5 \times$ larger than the LDA gaps. However, in a supercell of realistic 
dimensions the gap is suppressed to roughy $32 \mathrm{meV}$. The small variation of $\pm 0.1 \AA$ of the graphene- $h$-BN binding distance is important; without it the gap becomes smaller then $10 \mathrm{meV}$.

Besides the opening of a small band gap in the incommensurable $h$-BN|graphene structure, there is also a redistribution of electrons in the graphene sheet. In chapter 9 we study the formation of electron-hole puddles in graphene when its is placed on a $h$-BN substrate. We argue that the origin of this effect lies in the formation of an inhomogeneous dipole layer, which has the periodicity of the supercell. Locally the Dirac cone is pushed up or down relative to the common vacuum level. Therefore electrons will leave those regions where the cone is pushed upwards and they will occupy empty states in regions where the cone is lowered. The result is a graphene sheet which is $p$-type in some regions and $n$-type in other regions, i.e. electron-hole puddles. We attribute the puddle formation to the polar nature of the B-N bond and we therefore predict that these puddles will not form when graphene is placed on $\mathrm{MoS}_{2}$. 


\section{Samenvatting}

Het experimentele bewijs van de stabiliteit van een enkele grafeen laag in 2004 heeft geleid tot een nieuwe HYPE ${ }^{\dagger}$. Het vooruitzicht van HiglY Planar Electronics is aantrekkelijk voor de nanotechnologie: een geleidend, éen atoomlaag dik koolstof vlak met bijzondere elektronische eigenschappen als één van de belangrijkste bouwstenen voor zeer dunne en vlakke elektronische componenten. Men kan dromen van weerstanden, transistoren, diodes en condensatoren gemaakt van grafeen en andere 2D materialen. Aangezien deze materialen zeer dun zijn en de in-vlak $x y$-afmetingen veel groter zijn dan de $z$-afmeting, is het niet vreemd te veronderstellen dat de grensvlakken tussen de verschillende materialen een belangrijke factor zijn. In dit proefschrift worden de fysische processen die plaats vinden op dit soort grensvlakken bestudeerd. De primaire methode gebruikt in dit proefschrift om deze systemen te bestuderen is Dichtheids-Functionaal-Theorie (DFT), hoe het werkt en wat er mee kan worden berekend wordt uitgelegd in hoofdstuk 2. De tweede methode is het gebruik van fenomenologische modellen om de resultaten van de DFT berekeningen te interpreteren. Deze modellen geven inzicht door aan te tonen hoe de verschillende processen samen werken.

In hoofdstuk 3 beginnen we de studie door te kijken naar metaal|molecuul grensvlakken. Constante werkfunctie niveaus worden gemeten na afzetting van dezelfde soort moleculen op verschillende metalen. Deze niveaus worden pinning niveaus genoemd. De overdracht van elektronen tussen het metaaloppervlak en de moleculaire laag beïnvloedt de werkfunctie van het met moleculen bedekte oppervlak. Of elektronen worden overgedragen van de moleculen naar het metaal of omgekeerd, wordt bepaald door de electronenaffiniteit (of ionisatiepotentiaal), de opladingsenergie van de moleculen en de werkfunctie van het metaaloppervlak. Een model, dat op basis van een vlakke condensator is geconstrueerd en gecombineerd met parameters uit DFT-berekeningen, kan de pinningniveaus nauwkeurig voorspellen.

Hoofdstuk 4 toont een grafeen veld-effect-apparaat: een grafeen $\mid h$-BN heterostructuur op een koper oppervlak. De ladingsoverdracht tussen de laag grafeen en het koper oppervlak door de ultradunne $h$-BN laag wordt bestudeerd. Hoewel de onverstoorde werkfunctie van koper groter is dan die van grafeen, worden elektro-

\footnotetext{
${ }^{\dagger}$ Een passende afkorting waar mijn promotor Prof. Kelly mee kwam. In het Nederlands betekent het zoiets als uiterst vlakke elektronica.
} 
nen toch overgedragen van koper naar grafeen. Dit resulteert in een grafeen laag die intrinsiek $n$-type gedoteerd is. Het blijkt dat een aanzienlijke dipool gevormd wordt op het $\mathrm{Cu}(111) \mid h$-BN grensvlak en dat maakt de effectieve werkfunctie van het koper oppervlak kleiner dan die van grafeen. De dotering kan worden omgekeerd en verhoogd door het aanleggen van een elektrisch veld over de meerlagige structuur. Het dopingniveau van grafeen is gemodelleerd door middel van het vlakke condensator model. Toevoeging van termen overeenkomstig met de grensvlak effecten in de heterostructuur, resulteert in een model dat het d.m.v. DFT berekende doteringsniveau correct beschrijft. In hoofdstuk 5 wordt het koper oppervlak vervangen door een set van andere overgangsmetalen. Het blijkt dat de potentiaalstappen behorende bij de metaal $\mid h$-BN dipoollaag aanzienlijk zijn en verschillend zijn voor alle hier onderzochte metalen.

In hoofdstuk 6 wordt er een scherpe blik geworpen op de oorsprong van de grote potentiaal stappen die ontstaan op grensvlakken van zwak wisselwerkende metaal|isolator structuren. De metaal $\mid h$-BN structuur wordt als archetypisch voorbeeld en testgeval gebruikt. De meest gebruikte verklaring in het veld is dat de dipoollaag die gevormd wordt op deze grensvlakken veroorzaakt wordt door Pauli uitwisselingsafstoting, soms ook wel het "kussen effect" genoemd. Om dit te testen wordt een systeem geconstrueerd bestaande uit het geantrisymmetriseerd product van metaal- en $h$-BN golffuncties. Dit systeem heeft dan exacte Pauli uitwisselingsafstoting. Bij afstanden tussen het metaal en de isolator die typisch zijn voor fysisorptie, vormt uitwisselingsafstoting de belangrijkste bijdrage aan de potentiaalstap. Op deze en grotere afstanden resulteren chemische/van-der-Waals interacties in significante wijzigingen van de lokale elektronendichtheid, maar vormen een relatief kleine bijdrage aan de potentiaalstap. Op kortere afstanden verkleinen chemische bindingsinteracties de potentiaalstap.

De grote potentiaalstappen die gevonden worden op de grensvlakken van metaal $h$-BN structuren zijn belangrijk voor de energieniveau oplijning in metaal|2D materiaal heterostructuren. In hoofdstuk 7 worden de Schottky barrières van metaal $\mid h-\mathrm{BN}$ structuren berekend met behulp van DFT. De barrière kan worden gemodelleerd door middel van vacuümniveau oplijning van de energieniveaus van het metaal en het $h$-BN en deze vervolgens te verschuiven met een dipoolstap. De invloed van de potentiaalstap op de metaal- $h$-BN bindingsafstand is vrijwel hetzelfde voor alle metalen waarop $h$-BN fysisorpt. De potentiaalstap is daarom gefit aan een geparametriseerde functie die gebruikt kan worden voor alle metalen uit dit hoofdstuk.

In de laatste twee hoofdstukken worden de grensvlak-effecten in zwak gebonden 2D materialen bestudeerd. In hoofdstuk 8 is grafeen geplaatst op een oppervlak van $h$-BN, dat een vergelijkbare vlakke kristalstructuur heeft met een net iets $(\sim 1,8 \%)$ grotere roosterconstante. Vanwege de zwakke van-der-Waals-binding tussen deze twee lagen worden de roosters niet gelijkmazig. De kosten van het uitrekken van het grafeen of het samenduwen van $h$-BN wegen niet op tegen de energiewinst bij binding. Hierdoor ontstaat een supercelstructuur met een veel grotere periode. De effecten hiervan worden waargenomen als een moiré patroon in STM experimenten. 
De grootte van deze supercellen wordt bepaald door de hoek tussen het grafeen en het $h$-BN rooster en door het verschil in roosterconstante. Een realistische supercel is zo groot dat het buiten de mogelijkheden van huidige DFT implementaties ligt. Daarom is er een tight-binding model geconstrueerd, dat wordt geparametriseerd door het te fitten aan DFT resultaten gebaseerd op commensurabele grafeen $\mid h$-BN structuren. GW berekeningen zijn uitgevoerd om het effect van veel-deeltjes interacties op het de door $h$-BN geïnduceerde bandkloof in grafeen te bepalen. Het effect is enorm, de GW bandkloven in de grafeen $\mid h$-BN eenheidscel zijn ongeveer $5 \times$ groter dan de LDA bandkloven. Echter, in een supercel van realistische afmetingen wordt de bandkloof onderdrukt en blijft er slechts $32 \mathrm{meV}$ van over. De kleine variatie van $\pm 0.1 \AA$ in de grafeen- $h$-BN bindingsafstand in de supercel is belangrijk; zonder de variatie is de bandkloof kleiner dan $10 \mathrm{meV}$.

Naast de opening van een kleine bandkloof in de incommensurabele $h$-BN|grafeen structuur, is er een herverdeling van elektronen in het grafeen vlak. In hoofdstuk 9 bestuderen we de vorming van elektron-gat-poelen in grafeen geplaatst op een $h$ BN substraat. We stellen dat de oorzaak van dit effect ligt in de vorming van een inhomogene dipoollaag, met de periodiciteit van de supercel. Lokaal wordt dan de Dirac-kegel omhoog of omlaag geduwd ten opzichte van het gemeenschappelijke vacuümniveau. Daarom zullen elektronen uit regio's waar de kegel omhoog wordt geduwd verplaatsen naar regio's waar de kegel wordt verlaagd. Hier bezetten zij dan toestanden die voorheen onbezet waren. Het resultaat is een laag grafeen dat in sommige regionen $p$-type is en in andere regionen $n$-type is, er zijn elektron-gatpoelen zijn ontstaan. Het ontstaan van deze poelen wordt toegeschreven aan het polaire karakter van de B-N binding. Op basis hiervan wordt voorspelt dat deze poelen zich niet vormen in grafeen wanneer het wordt geplaatst op $\mathrm{MoS}_{2}$. 


\section{Acknowledgements/Dankwoord}

When I started in Enschede as a young physics student, I would not have believed that ten years later I would write the acknowledgements for my $\mathrm{PhD}$ thesis. This time would have been much less enjoyable without the heartily welcome I have received (throughout the years) from the people at the University of Twente and from the citizens of Enschede. To all those people I would like to say: "good goan" and "henig an".

In May 2009 I finished my master thesis in the group of my current day promotor Paul Kelly. At that time I decided not to start a PhD project and left Enschede to explore working at TNO. However, I realized that that something was missing and what excitement Science had brought me. I contacted Paul and he offered me a PhD position. So, Paul, first of all, thank you for taking me back in and for providing me with the possibility to develop myself further as a scientist. Your keen scientific insight and very precise and accurate scientific work style have helped me a lot. I most certainly hope that some of that has rubbed off on me. I will remember the long discussions we had whenever you came into the office with a new, and almost always, good idea. Sometimes it seemed that our roles were reversed and I had to hit the brakes and be pragmatic. I have very much enjoyed this open way of working with you and am very grateful for it.

Geert, thank you for being my supervisor. You are the best teacher in the physics department and an excellent scientist. Together we have worked in the EU project Minotor. You have guided me trough the maze of presentations, deliverables and milestones and most of the times shielded me off from these things. For that I am really thankful. I admire your quick deductive skills, your ability to see the bigger picture, your patience and your friendly attitude. I have profited so much from these traits in the last four years. Especially when I got stuck somewhere and you let me find out the cause by asking me the right questions.

I would like to thank my $\mathrm{PhD}$ committee, consisting of my promotors, Kristian Thygesen, Bart van Wees, Petr Khomyakov, Bene Poelsema, Hans Hilgenkamp and Han Gardeniers for taking the time to read this thesis and for making it possible for me to defend it in public.

Petr, the work that I have done under your supervision for my master thesis was the seed from which this PhD thesis has grown. Thank you for your continued 
collaboration. Together we have sharpened the story from a master thesis into two nice publications in Nano Letters and PRB. I am pleased that you are in the defence committee and that you will read the work that is new to you. I wish you all the best!

I would like to thank Mats Fahlman and Michel de Jong for our collaboration within the Minotor project. The agreement between your experimental work and our calculations on metal-organic interfaces and the discussions on the interpretation of the data have been very stimulating.

My colleagues and friends of the CMS group have been a continues source of support. Els, thank you for your secretary work and for discussing the matches of FC Twente with me. Unfortunately we had to miss you the last half year, I hope everything will turn for the better for you. Thank you Carin for helping me with the administrative procedures. I hope you enjoyed the time you were in our group and I wish you the all best at your new group. Taher, collaborating with you for the last two years has been very exciting. The quest for the graphene band gap lead us from no gap, to puddles, to a small GW gap. Your TB code has a lot of potential, I expect that you can still get many nice results from it! Rien, thank you for being a good friend and colleague. I have enjoyed talking with you in the coffee breaks, on conferences and outside of work. All the best to you. Nirmal and Jayita, thanks for the many lunch and coffee breaks that we shared. The Indian chicken legs at your place are definitely the best! Both Rien and Nirmal, thanks for your help in the writing process. Mojtaba, good luck with the Schottky barriers. Yi and Zhe, thanks for your positive and constructive attitude. I wish you all the best in Germany and in your future careers. Deniz, for being my buddy and collaborator in the Minotor project, both in the office as well as on conferences. Zhicheng, we have shared an office for three years. Thank you for the many interesting discussions. Elmer, it has been a joy to be your supervisor for your master thesis. I am happy that you have found a $\mathrm{PhD}$ position related to graphene at the UT. I wish you and Irma all the best! Thank you Jenny, for reading the introduction of this thesis and for providing suggestions to make it more accessible. Unna, for brightening up the atmosphere in my office when the desk opposite to me was vacant. Anton, thank you for the times you solved my coding problems. I hope you will be able to defend your thesis very soon. Diana, it has been a bumpy ride, that is for sure, but I would not have missed it for the world. In the last couple of years you have become a very dear friend. Muchas gracias for playing squash, having diner, cooking, watching movies and talking with me. You have widened my horizon on a personal level. Keep up the good thesis writing work and keep your goals clear. You will definitely make it. All the best to you and Ireneus.

Erik and Richard, thank you for being my paranimfs at the defence. Erik, we have known each other from high school. I experienced the joy of physics research for the first time when we tried to model the trajectory of a boomerang for our high school final project. Richard, we have known each other from the first day at the UT. Together we have studied, worked and struggled trough many courses. In 2011 I 
was your paranimf, I am very happy that you will come all the way form the USA to return the favor. To be able to defend this thesis with you two at my side is a honor.

\section{Er is meer dan werk alleen. Een goede basis is de start van elk resultaat:}

Met veel plezier heb ik mijzelf naast het onderzoek ingezet als leiding bij Scouting ' $t$ Volbert in Enschede. Elke zaterdag aan de slag met de scouts: vuur stoken, pionieren, kamperen en nog veel meer. Het maakt het mogelijk om de ingewikkelde materie even te laten voor wat het is en weer te aarden. Dank aan mijn leidingteam: Tim, Mark, Rob en Douwe! Jullie waren er trouw elke week voor een biertje, een lolletje of een schouderklop. Dank aan Johannes en Leanne, jullie zijn gewoon erg tof! Sanne, Kirsten, Noortje en Donnee, die "kippenboom" moeten we nog maar eens gaan maken. Tijs, succes in Rotterdam. Martijn, bedankt voor de vele aardige woorden. Evelien, bedankt voor de spelletjes en het mij niet laten winnen. Misschien kunnen we nog eens samen koken? Dank aan iedereen van 't Volbert, samen maken jullie de gaafste scoutinggroep in de regio!

Bedankt Hopman Masselman Groep en al mijn vrienden daarom heen. Dank voor de vriendschap door de jaren heen, ook al was ik niet meer iedere zaterdag op de Sparrenlaan: Auke, Joost, Peter, Reinier en Tjeerd. Martin, bedankt voor de hikes in Zweden (na de conferentie) en tijdens de Herfsthike. Daan, bedankt voor alle gezellige squashavonden.

Bedankt ds. Bregman, Reinier, Suzanne, Remco, Linda en Haro, samen hebben we ons mogen verdiepen in ons geloof met een mooi gevolg. Henk en Niek, dank dat jullie daar bij wilden zijn.

Tot slot, dank aan mijn vader en moeder, voor al jullie vertrouwen, liefde, aanmoediging, wijze woorden en goede voorbeeld. Dank ook aan mijn broer en zus, voor de gezelligheid, het spel en de aandacht. Beste Ben, Annette, Rob, Marijke en hond Tessa $t$, zonder jullie steun was dit proefschrift er niet geweest.

Menno Bokdam 


\section{Publications}

1. Fermi level pinning by integer charge transfer at electrode-organic semiconductor interfaces,

M. Bokdam, D. Cakir, G. Brocks,

Applied Physics Letters 98, 113303 (2011),

see Chapter 3 of this thesis.

2. Electrostatic Doping of Graphene through Ultrathin Hexagonal Boron Nitride Films,

M. Bokdam, P.A. Khomyakov, G. Brocks, ZC. Zhong, P.J. Kelly,

Nano Letters 11, 4631-4635 (2011),

see Chapter 4 of this thesis.

3. Modeling charge transfer at organic donor-acceptor semiconductor interfaces, D. Cakir, M. Bokdam, M.P. de Jong, M. Fahlman, G. Brocks, Applied Physics Letters 100, 203302 (2012).

4. Charge equilibration and potential steps in organic semiconductor multilayers, G. Brocks, D. Cakir, M. Bokdam, M.P. de Jong, M. Fahlman Organic Electronics 13, 1793-1801 (2012).

5. Field effect doping of graphene in metal|dielectric|graphene heterostructures: A model based upon first-principles calculations, M. Bokdam, P.A. Khomyakov, G. Brocks, P.J. Kelly, Physical Review B 87, 075414 (2013), see Chapter 5 of this thesis.

6. Large potential steps at weakly interacting metal|insulator interfaces, M. Bokdam, G. Brocks, to be submitted, (2013), see Chapter 6 of this thesis.

7. Schottky barriers at metal|hexagonal boron nitride interfaces: a first principles study, M. Bokdam, G. Brocks, P.J. Kelly to be submitted, (2013), see Chapter 7 of this thesis. 
8. Band gaps in incommensurable Graphene on hexagonal Boron-Nitride, M. Bokdam, T. Amlaki, G. Brocks, P.J. Kelly

to be submitted, (2013),

see Chapter 8 of this thesis.

9. Intrinsic electron-hole puddles in Graphene on hexagonal Boron-Nitride, M. Bokdam, T. Amlaki, G. Brocks, P.J. Kelly

to be submitted, (2013),

see Chapter 9 of this thesis. 


\section{Curriculum vitae}

INDIVIDUAL

Name: $\quad$ Menno Bokdam

Date of birth: 10th of October, 1985

Place of birth: Soest, The Netherlands

\section{EDUCATION}

2009-2013 Ph.D. in Science

Title: "Charge transfer and redistribution at interfaces between metals and 2D materials"

Computational Materials Science

University of Twente, Enschede, The Netherlands

2006-2009 M.Sc. in Applied Physics

Title: "First principles study of graphene|boron nitride|

metal heterostructures"

Computational Materials Science

University of Twente, Enschede, The Netherlands

2003-2006 B.Sc. in Applied Physics

Title: "Experiments with an atomic force microscope to determine the existence of surface nanobubbles"

Solid State Physics \& Physics of Fluids

University of Twente, Enschede, The Netherlands

1997-2003 High school, VWO level

Title: "De baan van een boemerang"

Griftland College, Soest, The Netherlands 


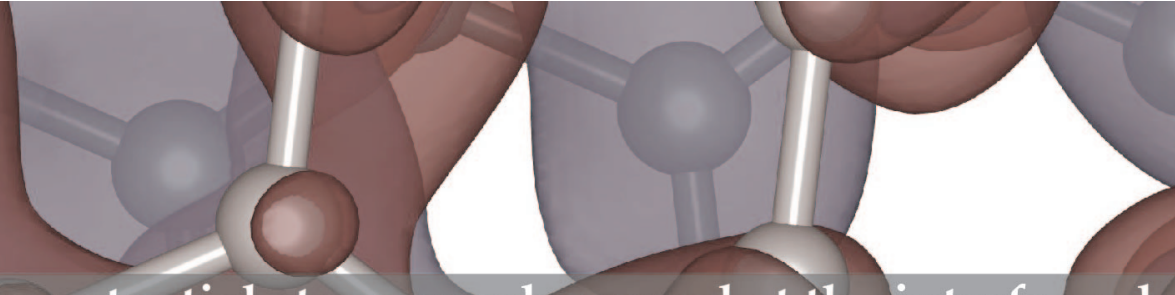

Large potential steps are obsevered at the interfaces between metals and novel 2D materials. They can lower the work function by more then $1 \mathrm{eV}$, even when the two parts are only weakly interacting. In this thesis the transfer and redistribution of electrons in metal $12 \mathrm{D}$ material heterostructures are studied by means of first principles methods and analyzed by using phenomenological models. Special attention is paid to the incommensurable interface structure of hexagonal Boron-Nitride and Graphene. It is shown that a small bandgap is opened in graphene when the angle between the two lattices is small. Larger angles lead to electron-hole puddles.

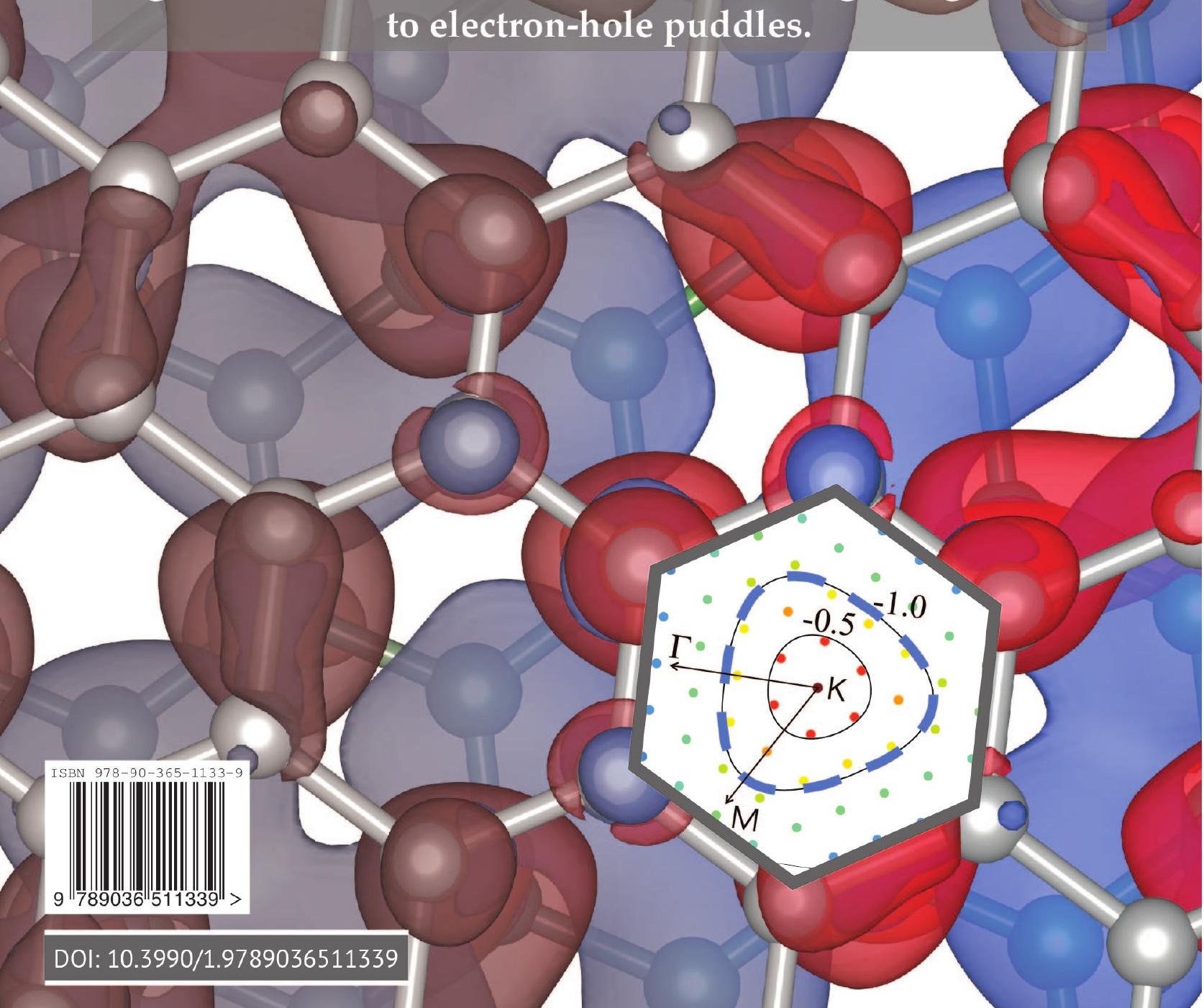

